\title{
Background noise
}

Citation for published version (APA):

Fuller, T. E. (2021). Background noise: clinical guidelines, assessment \& CBT for disabling tinnitus.

[Doctoral Thesis, Maastricht University]. Ridderprint. https://doi.org/10.26481/dis.20210113tf

Document status and date:

Published: 01/01/2021

DOI:

10.26481/dis.20210113tf

Document Version:

Publisher's PDF, also known as Version of record

\section{Please check the document version of this publication:}

- A submitted manuscript is the version of the article upon submission and before peer-review. There can be important differences between the submitted version and the official published version of record.

People interested in the research are advised to contact the author for the final version of the publication, or visit the DOI to the publisher's website.

- The final author version and the galley proof are versions of the publication after peer review.

- The final published version features the final layout of the paper including the volume, issue and page numbers.

Link to publication

\footnotetext{
General rights rights.

- You may freely distribute the URL identifying the publication in the public portal. please follow below link for the End User Agreement:

www.umlib.nl/taverne-license

Take down policy

If you believe that this document breaches copyright please contact us at:

repository@maastrichtuniversity.nl

providing details and we will investigate your claim.
}

Copyright and moral rights for the publications made accessible in the public portal are retained by the authors and/or other copyright owners and it is a condition of accessing publications that users recognise and abide by the legal requirements associated with these

- Users may download and print one copy of any publication from the public portal for the purpose of private study or research.

- You may not further distribute the material or use it for any profit-making activity or commercial gain

If the publication is distributed under the terms of Article $25 \mathrm{fa}$ of the Dutch Copyright Act, indicated by the "Taverne" license above, 
(1) :

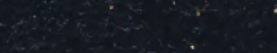

BACKGROUND

NOISE

CLINICAL GUIDELINES, ASSESSMENT \&. CBT FOR DISABLING TINNITUS

Thomas E. Fuller

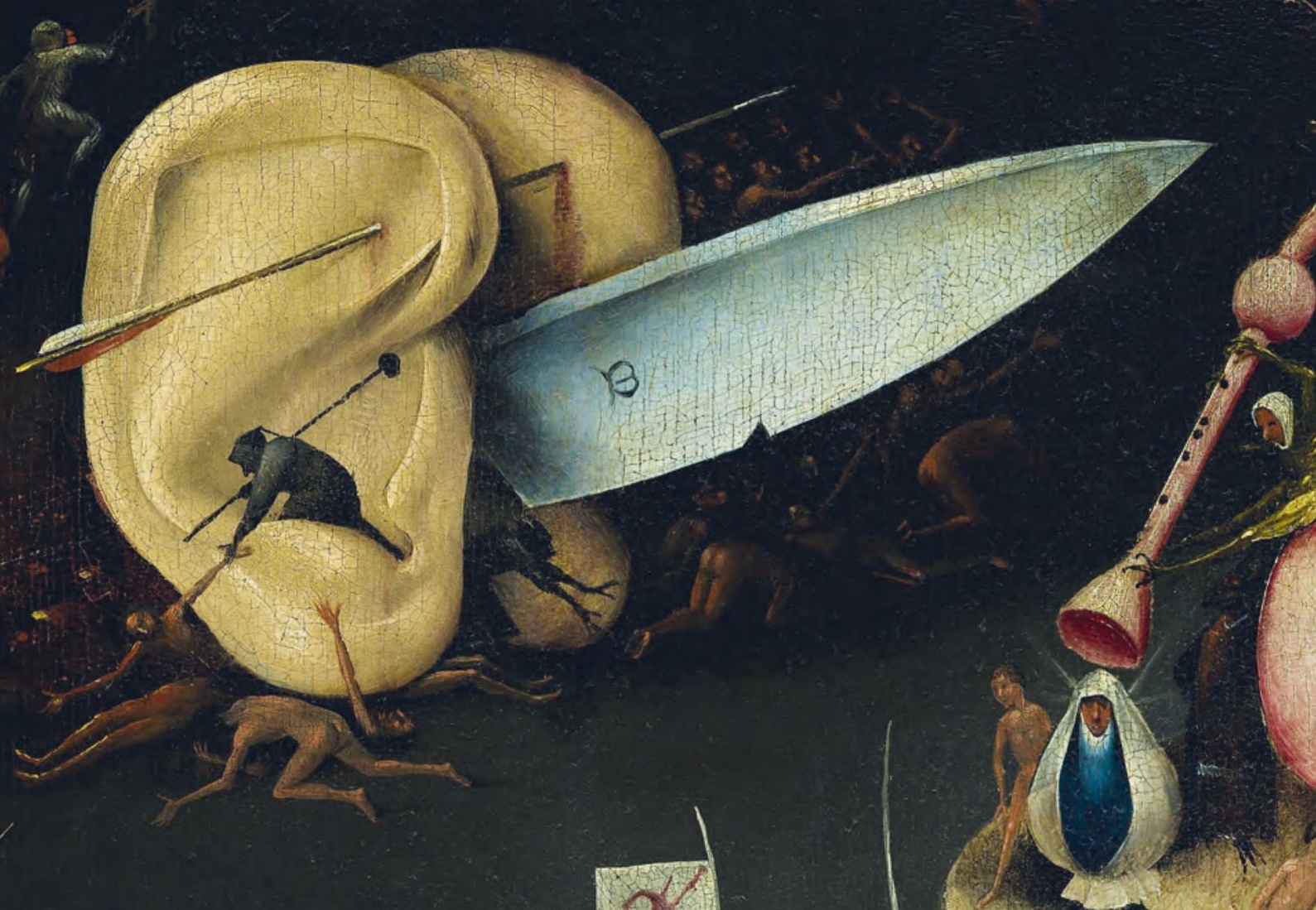





\section{BACKGROUND NOISE \\ CLINICAL GUIDELINES, ASSESSMENT \& CBT FOR DISABLING TINNITUS}

Thomas E. Fuller 


\section{BACKGROUND NOISE, THOMAS FULLER}

\section{ISBN/EAN: 978-94-6416-270-7}

Cover image by Hieronymus Bosch, The Garden of Earthly Delights. circa 1480-1490

Layout and design by Yasmin Katlich, persoonlijkproefschrift.nl

Printing by Ridderprint | www.ridderprint.nl

Copyright (C) 2020 THOMAS FULLER

All rights reserved. No part of this thesis may be reproduced, stored or transmitted in any way or by any means without the prior permission of the author, or when applicable, of the publishers of the scientific papers. 


\section{BACKGROUND NOISE}

CLINICAL GUIDELINES, ASSESSMENT \& CBT FOR DISABLING TINNITUS

\section{DISSERTATION}

to obtain the degree of Doctor at the Maastricht University, on the authority of the Rector Magnificus, Prof.dr. Rianne M. Letschert in accordance with the decision of the Board of Deans,

to be defended in public on Wednesday 13th January 2021, at12:00.

By

Thomas Edward Fuller 
Promoter: Professor Dr. Johan W. S. Vlaeyen

Co-Promoter: Dr. Rilana Cima

\author{
Members of the Assessment Committee \\ Professor Dr. Madelon Peters (Chair) \\ Dr. Amr El Refaie \\ Dr. Laurence McKenna \\ Professor Dr. Jeanine Verbunt
}

This research was financially supported by SWOL Limburgs Fonds voor Revalidatie and Netherlands Organisation for Health Research and Development (ZonMW), the Netherlands, Research programme: Health Care Efficiency, Subprogramme: Effects \& Costs, Grant number: 945-07-715 awarded to Rilana Cima. 


\section{TABLE OF CONTENTS}

$\begin{array}{lll}\text { Chapter } 1 & \text { Introduction } & 7\end{array}$

Chapter 2 Systematic review of clinical guidelines for the assessment and management of chronic tinnitus $\quad 39$

Chapter 3 Systematic review and meta-analysis of CBT for tinnitus in adults $\quad \mathbf{7 1}$

Chapter 4 The Fear of Tinnitus Questionnaire: Toward a valid and reliable means of assessing fear in adults with tinnitus

Chapter 5 Pragmatic uncontrolled study of specialized cognitive behavioural therapy for adults with chronic tinnitus $\quad 215$

$\begin{array}{lll}\text { Chapter } 6 & \text { Discussion } & 239\end{array}$

Valorisation addendum $\quad 273$

Acknowledgements $\quad 277$

CV 280 

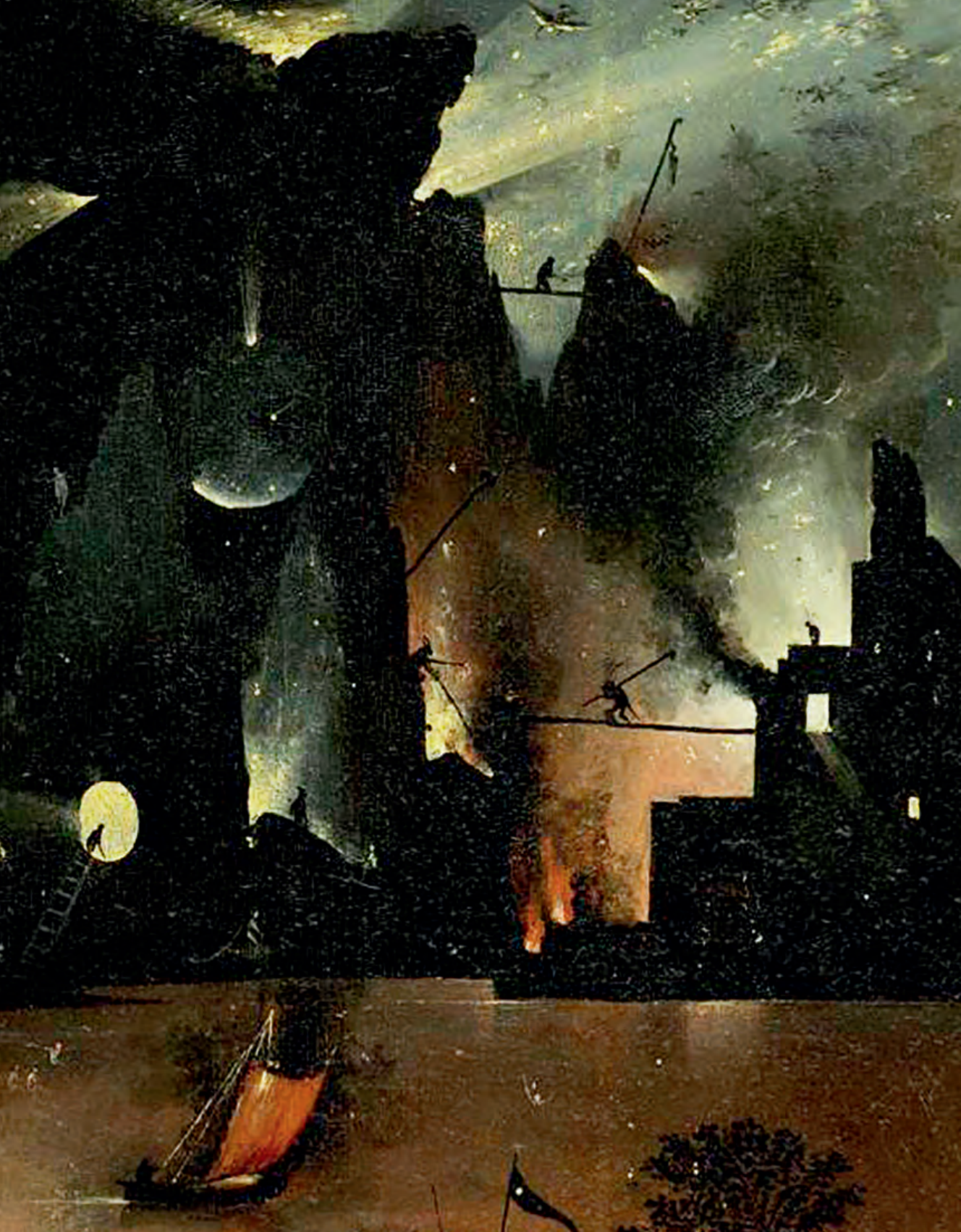

$\rightarrow-2=$ 


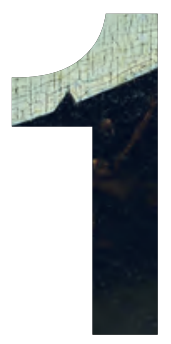

\section{INTRODUCTION}


From conversation, music, and traffic, to those encountered in nature, sounds are ever-present, whether we hear them or not. Although acoustic properties of externally generated sound can be measured in, for example, Hertz and decibels, hearing is ultimately a subjective experience (Lotto \& Holt, 2011). Further, as sounds are filtered or selected from the environment, psychological factors such as attention, motivation, and previous experience all influence how sound is interpreted and experienced (Snyder, Gregg, Weintraub, \& Alain, 2012). For example, negative emotion can contribute to people perceiving sounds to be louder than if they are in a "neutral" mood (Asutay \& Västfjäll, 2012; Siegel \& Stefanucci, 2011). But what if sounds are perceived in the absence of an external source, and what if these sounds persist? In that case, and especially if the sounds are not voices or music, it is likely that the individual is experiencing what is called "tinnitus".

Tinnitus, derived from the Latin tinnire (meaning to ring) (Baguley, McFerran, \& Hall, 2013) presents numerous academic and therapeutic challenges to researchers and clinicians. Tinnitus can be "objective" when detectable to an observer. More commonly, tinnitus is subjective and the "sound" or experience of it, is unable to be shared directly with other people. In its subjective form, psychological processes are also likely to influence interpretations of tinnitus, while precluding the possibility of measuring it directly in terms of Hertz or decibels. While the etymology of the word tinnitus is clear, there is no consensus on how the tinnitus percept should be defined from a research or clinical perspective (e.g., Kroner-Herwig, Frenzel, Fritsche, Schilkowsky, \& Esser, 2003; Philippot, Nef, Clauw, de Romree, \& Segal, 2012). Definitions of tinnitus do share or make reference to the absence of an identifiable source of the perceived sound. Beyond that though, there are marked differences between existing definitions, as illustrated in Table 1.

In this dissertation, we are primarily concerned with the assessment and treatment of people suffering from or seeking help with managing the interference in daily activities that can accompany subjective tinnitus. Thus a definition that also includes reference to its potential impact is most relevant and useful here. After all, if tinnitus does not significantly, negatively impact peoples' lives, then they would not require a treatment to improve their mood and functioning. Given this distinction, we will be focussing on chronic disabling tinnitus, which we define as: "The phantom perception of sound coinciding (or associated) with an aversive emotional experience that persists for at least three-months". This definition is closest to that of Hoare, Edmondson-Jones, Gander, and Hall (2014) and Cima (2018) (Table 1.), in that these authors also refer the absence of an identifiable source and the aversive consequences of perceiving the sound. However, it adds a temporal indication to differentiate between acute and chronic disabling tinnitus. Additionally, it uses the word "disabling" in place of "bothersome" which is often used in tinnitus literature (e.g. Cima, 2018) and in doing so aligns with the International Classification of Functioning, Disability and Health (ICF) (World Health Organization, 2001) 
Tinnitus in this this definition is conceptualised as an interoceptive stimulus. Moreover, "perceiving a sound" does not refer to the commonly understood meaning of hearing sounds as soundwaves interpreted by the auditory system. The duration for which we specify that tinnitus is experienced as being chronic is admittedly arbitrary since there is no research that reliably distinguishes or differentiates between tinnitus of particular "acute" or "chronic" durations (Wallhäusser-Franke et al., 2017). The main point though, regardless of duration, is that the tinnitus has not spontaneously remitted for a considerable time period. Our definition of chronic disabling tinnitus does not refer to any acoustic qualities of tinnitus. For example, it does not specify, address or consider whether the tinnitus is perceived constantly or intermittently; whether it is the same or varied with regard to sound quality, or if it is perceived at a constant or fluctuating loudness; or whether it is one class of symptoms with many subtypes. The acoustic qualities of tinnitus are important for diagnostic purposes, but less so in defining disabling subjective tinnitus. Besides, research has already established that the perceived perceptual characteristics of tinnitus, such as loudness, are weak predictors of levels of annoyance (G. Andersson, 2003; Hiller \& Goebel, 2006), distress and interference in daily life activities (Cima, Crombez, \& Vlaeyen, 2011).

\section{Epidemiology and societal impact of chronic disabling tinnitus}

Estimates of the prevalence of chronic disabling tinnitus based on UK, Japanese and Korean studies vary but typically range between 1 and $3 \%$ of the population (Davis \& El Refaie, 2000; Fujii et al., 2011; Kim et al., 2015). By way of context, estimates of the prevalence of non-disabling chronic tinnitus range between 15 and $20 \%$ of the adult population (Davis \& El Refaie, 2000; Kim et al., 2015). In the Netherlands, the prevalence of chronic disabling tinnitus is associated with high personal, societal and economic costs. A cost of illness study by Maes, Cima, Vlaeyen, Anteunis, and Joore (2013) estimated that the overall costs of disabling tinnitus was approximately $€ 6.8$ billion per year, which equated to $2.3 \%$ of total health care expenditure in 2009. Interestingly, indirect productivity-related costs comprised a greater proportion of the total direct costs than those associated with healthcare. This is also borne out in results from a recent study of the healthcare costs associated with disabling tinnitus in the UK which estimated that it costs the National Healthcare Service (NHS) $£ 750$ million per year to treat tinnitus patients, with the societal cost amounting to approximately £2.7 billion per year (Stockdale et al., 2017). Although the two studies used different data from which to make their calculations, they indicate that that an effective, affordable treatment could be both economically and socially advantageous. 
Chapter 1

Table 1. Examples of Definitions of Tinnitus and The Attributes to Which They Refer.

\begin{tabular}{|c|c|c|c|c|}
\hline Definition & Source & Measurable Occurrence & $\begin{array}{l}\text { Quality } \\
\text { of sound }\end{array}$ & Impact \\
\hline $\begin{array}{l}\text { "A common and distressing condition } \\
\text { that is typically characterised by the } \\
\text { perceived sensation of sound in the } \\
\text { absence of an external stimulus. Usually } \\
\text { tinnitus is perceived as a continuous } \\
\text { buzzing, hissing or ringing in the } \\
\text { ears...." (p. 1635; Langguth, 2011) }\end{array}$ & $x$ & $x$ & $x$ & $x$ \\
\hline $\begin{array}{l}\text { "an auditory phantom perception, and } \\
\text { therefore cannot be associated with } \\
\text { any sound measurement" (p. 216; } \\
\text { Jastreboff, Hazell, \& Graham, 1994) }\end{array}$ & $x$ & $x$ & & \\
\hline
\end{tabular}

\begin{tabular}{lll}
\hline $\begin{array}{l}\text { "Tinnitus refers to a sensation of sound } \quad \mathrm{X} \\
\text { not stimulated by a simultaneous }\end{array}$ & & \\
acoustical or electrical signal" (p. 381; & & \\
Kroner-Herwig et al., 2003) & & \\
\hline " the conscious perception of an & $\mathrm{X}$
\end{tabular}

"...the conscious perception of an auditory sensation in the absence of a corresponding external stimulus...

-descriptions of hissing, sizzling,

and ringing are common..." (p. 1600;

Baguley, McFerran, et al., 2013)

"Tinnitus is characterized by two major $\mathrm{X}$ $x$ components, the phantom percept of sound, and the emotional reaction or perceived threat associated with that sound." (p. 1; Hoare et al., 2014)

"The symptom itself, tinnitus aurium, $\mathrm{X}$ $x$ can be defined as the phantom perception of continuous sound or noise in the absence of an external (or adequate) source..... A bothersome tinnitus (causing illness) might be best described as a negative auditory experience coinciding with aversive emotional reactivity, associated with, or described in terms of, actual or potential (bodily or psychological) harm." (p. 369; Cima, 2018)

The phantom perception of sound co$x$ $x$ inciding (or associated) with an aversive emotional experience that persists for at least three-months. (This thesis) 


\section{Pathophysiology of tinnitus}

The precise underlying physiological mechanisms of tinnitus are unknown and the variability in symptoms that people experience is large (Cederroth et al., 2019). For example, it is well documented that many people (e.g., estimates of 63\%) with chronic disabling tinnitus have some degree of hearing loss (Martines et al., 2010; Ratnayake, Jayarajan, \& Bartlett, 2009), that the chances of having tinnitus increase substantially with hearing loss (Nondahl et al., 2011), and that the prevalence of disabling tinnitus increases with the severity of hearing loss (Martines et al., 2010). However, not all people with hearing loss are able to perceive tinnitus and not all people with tinnitus have clinically significant hearing loss (Baguley, McFerran, et al., 2013).

Regardless, evidence from both animal models and studies of human tinnitus sufferers has suggested that cochlear damage might be a necessary but not sufficient condition for tinnitus to occur. Instead, it is proposed, that tinnitus is generated by changes that take place in central auditory pathways when auditory neurons lose their input from the ear (Noreña, 2011). In particular, neural plasticity through, for example increased spontaneous activity and neural gain in deafferented central auditory structures and changes in network behaviour in non-auditory brain regions, is thought to underlie this phenomenon (Eggermont \& Roberts, 2014; Elgoyhen, Langguth, De Ridder, \& Vanneste, 2015). In addition, non-auditory related areas of the brain, such as those associated with awareness, memory (De Ridder, Elgoyhen, Romo, \& Langguth, 2011; De Ridder et al., 2014), and emotion (Rauschecker, Leaver, \& Mühlau, 2010), contribute to pathophysiological explanations of responses to tinnitus.

\section{Psychological mechanisms of disabling tinnitus}

It is unclear as to why some people but not others progress from having an acute tinnitus to chronic tinnitus that can cause disruption in all facets of life. Sufferers of tinnitus report experiencing disruption to sleep, concentration, and ability to engage in conversation, interference in work and social functioning, and for some, suicidal ideation (G. Andersson \& Edvinsson, 2008; Joo, Han, \& Park, 2015; Tyler \& Baker, 1983). Psychological models - which we will briefly describe below - have been proposed to explain potential responses to tinnitus, but none has universal endorsement. Biological (animal) models of tinnitus have been proposed but are not fully able to explain the physical mechanisms of tinnitus. Furthermore, for example, one would expect that if a biological account of tinnitus was sufficient, the levels of tinnitus-related distress and impairment would correlate strongly with loss of hair cells, lesions in the brain or malfunction in the auditory cortex. This is not the case.

In general, a lack of conclusive evidence and consensus on many aspects of chronic tinnitus - from the physiological mechanisms that cause and maintain the "auditory phenomenon", to how best to manage the interference and distress it can cause epitomises the current state of the field. This however is not to say that progress and developments in the understanding of chronic tinnitus have not been made, but instead to highlight that the scientific field studying tinnitus is relatively "young" and that there is much to discover with regard to its aetiology, assessment and treatment. In this context, we expect that the studies within this dissertation will provide reference points or the foundations from which further research and clinical work can evolve. 


\section{Psychological models of tinnitus distress}

Psychological models of tinnitus are appealing since, for example, the level of tinnitusrelated distress correlates weakly with psychoacoustic measures of loudness (G. Andersson, 2003; Kuk, Tyler, Russell, \& Jordan, 1990) and moderately with self-reported loudness (Hiller \& Goebel, 2006; Wallhäusser-Franke et al., 2012). Psychological models of tinnitus also have appeal in their own right through their ability to provide a better understanding of the development and maintenance of disabling tinnitus. Additionally, they can be used to generate possible solutions to reverting its disabling effects.

"Tinnitus-related distress" however as a concept (or outcome) is not usually defined in studies, cannot be objectively measured (Gerhard Andersson \& Westin, 2008) and is apparently used an umbrella term for aversive/unpleasant emotional responses specifically to tinnitus. For example in systematic reviews "tinnitus distress" is often used as the primary outcome (e.g., Hesser, Weise, Westin, \& Andersson, 2011) and assessed by self-report questionnaires such the Tinnitus Handicap Inventory (Newman, Jacobson, \& Spitzer, 1996), Tinnitus Questionnaire (Hallam, 2008; Hallam, Jakes, \& Hinchcliffe, 1988) or Tinnitus Functional Inventory (Meikle et al., 2012). As illustrated in Table 2, each measure is closely related, but frames questions differently, uses different response options and has a slightly different focus. It is also important to note that tinnitus-related distress is also viewed as being distinct from depression or anxiety which is often measured by questionnaires such as the Hospital Anxiety and Depression Scale (Zigmond \& Snaith, 1983) or Beck Depression Inventory (Beck, Epstein, Brown, \& Steer, 1988).

Table 2. Examples of Questionnaires Used to Measure Tinnitus-related Distress.

\begin{tabular}{|c|c|c|c|}
\hline Measures & Purpose & $\begin{array}{l}\text { Examples (and item } \\
\text { number) }\end{array}$ & Response options \\
\hline $\begin{array}{l}\text { Tinnitus Functional } \\
\text { Inventory (Meikle et } \\
\text { al., 2012) }\end{array}$ & $\begin{array}{l}\text { Designed to assess } \\
\text { the major negative } \\
\text { functional impacts from } \\
\text { tinnitus and as well as } \\
\text { being used for evaluat- } \\
\text { ing treatment effects. }\end{array}$ & $\begin{array}{l}\text { (5) How easy was it for } \\
\text { you to COPE with your } \\
\text { tinnitus? } \\
\text { (14) Over the PAST WEEK, } \\
\text { how much has your tinni- } \\
\text { tus interfered with... Your } \\
\text { ability to UNDERSTAND } \\
\text { PEOPLE who are talking? }\end{array}$ & $\begin{array}{l}0 \text { (very easy to cope) } \\
-10 \text { (impossible to } \\
\text { cope) Likert type } \\
\text { scale } \\
0 \text { (did not interfere) } \\
-10 \text { (completely } \\
\text { interfered) Likert- } \\
\text { type scale }\end{array}$ \\
\hline $\begin{array}{l}\text { Tinnitus Handicap } \\
\text { Inventory (Newman } \\
\text { et al., 1996) } \\
\end{array}$ & $\begin{array}{l}\text { A diagnostic and screen- } \\
\text { ing tool that measures } \\
\text { the impact and severity } \\
\text { of tinnitus on everyday } \\
\text { function. }\end{array}$ & $\begin{array}{l}\text { (3) Does your tinnitus } \\
\text { make you angry? } \\
\text { (15) Because of your tinnitus } \\
\text { is it difficult to read? }\end{array}$ & Yes, Sometimes, No \\
\hline $\begin{array}{l}\text { Tinnitus Question- } \\
\text { naire (Hallam, 2008; } \\
\text { Hallam et al., 1988) }\end{array}$ & $\begin{array}{l}\text { Measures psychological } \\
\text { aspects of tinnitus com- } \\
\text { plaints and distress }\end{array}$ & $\begin{array}{l}\text { (10) The way the noises } \\
\text { sound is really unpleasant. } \\
\text { (18) I have lost some of my } \\
\text { confidence because of the } \\
\text { noises. }\end{array}$ & $\begin{array}{l}\text { True, Partly True, } \\
\text { Not true. }\end{array}$ \\
\hline
\end{tabular}


Following, we briefly discuss four influential psychological models of tinnitus that underlie the development of and rationale for applying cognitive behavioural therapy (CBT) to tinnitus-related distress. The models are the: habituation model (Hallam, Rachman, \& Hinchcliffe, 1984); the neurophysiological model (Jastreboff, 1990; Jastreboff et al., 1994); a cognitive behavioural (CB) model of tinnitus (McKenna, Handscomb, Hoare, \& Hall, 2014); and, the Fear Avoidance (FA) model (Cima et al., 2011; Kleinstäuber et al., 2013). Each of the models' core assumptions, predictions and empirical support is briefly described in Table 3.

Table 3. Overview of Models of Tinnitus.

\begin{tabular}{|c|c|c|c|}
\hline Model & $\begin{array}{l}\text { Fundamental } \\
\text { assumptions }\end{array}$ & Core predictions & Empirical support \\
\hline $\begin{array}{l}\text { Habituation } \\
\text { model (Hallam } \\
\text { et al., 1984). }\end{array}$ & $\begin{array}{l}\text { That tinnitus is } \\
\text { stable, consistent or } \\
\text { constant enough to } \\
\text { enable learning pro- } \\
\text { cesses to account } \\
\text { for a decrease in } \\
\text { response to tinnitus, } \\
\text { i.e. habituation. }\end{array}$ & $\begin{array}{l}\text { If failures in habituation } \\
\text { occur when attentional } \\
\text { processes malfunction, } \\
\text { most often in situations of } \\
\text { heightened physiological } \\
\text { arousal, then relaxation } \\
\text { and other stress reduction } \\
\text { techniques should be used } \\
\text { as a treatment to facili- } \\
\text { tate the reappraisal of the } \\
\text { meaning of the tinnitus and } \\
\text { habituation. }\end{array}$ & $\begin{array}{l}\text { Limited empirical support } \\
\text { exists for the habituation } \\
\text { model (Baguley, Anders- } \\
\text { son, et al., 2013). }\end{array}$ \\
\hline $\begin{array}{l}\text { Neurophysio- } \\
\text { logical model } \\
\text { (Jastreboff, } \\
\text { 1990; Jastre- } \\
\text { boff, Brennan, } \\
\text { Coleman, \& } \\
\text { Sasaki, 1988) }\end{array}$ & $\begin{array}{l}\text { The perception of } \\
\text { tinnitus is paired, } \\
\text { through classi- } \\
\text { cal conditioning, } \\
\text { with an aversive } \\
\text { emotional reaction; } \\
\text { conscious negative } \\
\text { evaluation of tinnitus } \\
\text { is not required for } \\
\text { this process. The } \\
\text { model also assumes } \\
\text { that animal models } \\
\text { of tinnitus are valid } \\
\text { and applicable to } \\
\text { humans. }\end{array}$ & $\begin{array}{l}\text { If classical conditioning } \\
\text { accounts for the associa- } \\
\text { tion between tinnitus and } \\
\text { an aversive emotional ex- } \\
\text { perience, then presenting } \\
\text { the conditioned stimulus } \\
\text { without the unconditioned } \\
\text { stimulus (i.e., extinction) } \\
\text { would result in decreased } \\
\text { tinnitus distress. Jastreboff } \\
\text { proposed that through } \\
\text { counselling and sound } \\
\text { therapy, the learned asso- } \\
\text { ciation between tinnitus } \\
\text { and an aversive emotional } \\
\text { response is broken and ha- } \\
\text { bituation is made possible. }\end{array}$ & $\begin{array}{l}\text { The empirical basis for the } \\
\text { model is based on animal } \\
\text { models of tinnitus and } \\
\text { assumed to be applicable } \\
\text { to humans. Mixed evidence } \\
\text { of effectiveness of Tinnitus } \\
\text { Retraining Therapy (the } \\
\text { indicated therapeutic } \\
\text { treatment according to } \\
\text { Jastreboff) is available } \\
\text { (Bauer, Berry, \& Brozoski, } \\
\text { 2017; Phillips \& McFerran, } \\
\text { 2010). }\end{array}$ \\
\hline
\end{tabular}


Chapter 1

Table 3. Continued.

\begin{tabular}{|c|c|c|c|}
\hline Model & $\begin{array}{l}\text { Fundamental } \\
\text { assumptions }\end{array}$ & Core predictions & Empirical support \\
\hline $\begin{array}{l}\text { Cognitive } \\
\text { behavioural } \\
\text { model (McKen- } \\
\text { na et al., 2014) }\end{array}$ & $\begin{array}{l}\text { Negative automatic } \\
\text { evaluation of tinnitus } \\
\text { is primarily respon- } \\
\text { sible for the/a dys- } \\
\text { functional emotional } \\
\text { and behavioural } \\
\text { response to tinnitus. }\end{array}$ & $\begin{array}{l}\text { If negative automat- } \\
\text { s ic thoughts about and } \\
\text { appraisals of one's ability } \\
\text { to cope with tinnitus are } \\
\text { fundamentally responsible } \\
\text { for disabling tinnitus, then } \\
\text { cognitive and behavioural } \\
\text { treatments should focus } \\
\text { on this to achieve thera- } \\
\text { peutic benefits. }\end{array}$ & $\begin{array}{l}\text { McKenna et al. (2014) cite } \\
\text { numerous studies that } \\
\text { provide evidence for com- } \\
\text { ponents of the model (e.g. } \\
\text { the role of selective attention } \\
\text { in distorting the perception } \\
\text { of tinnitus) although the } \\
\text { studies themselves were not } \\
\text { designed to test the model } \\
\text { itself. Additionally, low to } \\
\text { moderate quality evidence } \\
\text { exists for the effectiveness of } \\
\text { CBT to reduce tinnitus-relat- } \\
\text { ed distress and interference } \\
\text { which indirectly provides } \\
\text { some support for the model } \\
\text { (Fuller et al., 2020). }\end{array}$ \\
\hline $\begin{array}{l}\text { Fear Avoidance } \\
\text { model of tinni- } \\
\text { tus (Cima et al., } \\
2011 \text {; Kleinstäu- } \\
\text { ber et al., 2013) }\end{array}$ & $\begin{array}{l}\text { Catastrophic } \\
\text { interpretations of } \\
\text { auditory stimuli and } \\
\text { associative learning } \\
\text { are responsible for a } \\
\text { cascade of emotion- } \\
\text { al and behavioural } \\
\text { reactions to tinnitus. } \\
\text { It is also assumed } \\
\text { that fear is the } \\
\text { dominant (or at least } \\
\text { primary) emotional } \\
\text { response to tinnitus. }\end{array}$ & $\begin{array}{l}\text { If classical and operant } \\
\text { mechanisms initiate and } \\
\text { maintain disabling tinnitus, } \\
\text { and fear is the primary } \\
\text { emotional response, then } \\
\text { - exposure therapy leading } \\
\text { to extinction will be an } \\
\text { effective treatment. Further, } \\
\text { if catastrophic (mis)interpre- } \\
\text { tations of tinnitus precip- } \\
\text { itates a cycle of negative } \\
\text { emotional and behavioural } \\
\text { responses then, interpreting } \\
\text { it as a neutral (even positive) } \\
\text { stimulus leads to a function- } \\
\text { al response to tinnitus. }\end{array}$ & $\begin{array}{l}\text { Some evidence for the role } \\
\text { of cognitive and emotional } \\
\text { processes within the Fear } \\
\text { Avoidance Model of tinnitus } \\
\text { exists (Blaesing \& Kroen- } \\
\text { er-Herwig, 2012; Cima, van } \\
\text { Breukelen, \& Vlaeyen, 2017; } \\
\text { Kleinstäuber et al., 2013). } \\
\text { Additionally, low to moder- } \\
\text { ate quality evidence exists } \\
\text { for the effectiveness of CBT } \\
\text { to reduce tinnitus-related } \\
\text { distress and interference } \\
\text { which indirectly provides } \\
\text { some support for the model } \\
\text { (Fuller et al., 2020). }\end{array}$ \\
\hline
\end{tabular}




\section{The habituation model of disabling tinnitus}

Habituation is a process whereby reaction(s) to a stimulus decrease in response to repeated presentations of the same stimulus (Bouton, 2016). Additionally, it is considered to be context- and individual-specific as well as influenced by and susceptible to indirect learning and motivational factors (De Paepe, Williams, \& Crombez, 2019). The principle of habituation was first applied to tinnitus in 1984 by Hallam and colleagues to explain reduction in tinnitus-related distress over time. It has some intuitive appeal as, for most people, it appears that repeated perception of the tinnitus leads to them learning that it is a stimulus not worthy of attentional resources; i.e. ultimately it , through repeated presentation, becomes a non-aversive stimulus (Hallam et al., 1984). Tinnitus-related distress according to this model occurs when there is a "failure" to habituate; that is attention (or some other response) continues to be directed to tinnitus despite its repeated, benign, occurrence.

Operant conditioning, a form of associative learning where the frequency of a behaviour is increased through rewards (i.e., positive or negative reinforcement) or an unwanted behaviour decreased through positive or negative punishment (Skinner, 1938), was later included into the habituation model (referred to as "operant" factors in Figure 1). Operant factors were added to the model to account for learning and emergence of avoidant behaviours (Kroner-Herwig et al., 2003). For example, negative reinforcement (i.e., where an aversive stimulus is removed), is thought to explain an increase in or maintenance of avoidance behaviours as people with tinnitus might fear entering a noisy environment. Fear or worry associated with noisy places initiates the fight or flight response (i.e. autonomic nervous system arousal), and hence a person gains relief by avoiding or leaving the environment quickly to experience the reinforcing/corresponding decrease in fear-related arousal. Thus, avoiding noisy places becomes a strategy to avoid or minimise the chances or levels of fear or worry associated with anticipated worsening of tinnitus. This avoidance provides immediate relief but in the longer-term can, for example, lead to social isolation and/or loss of participation in activities that were previously enjoyed.

To manage tinnitus distress, according to this model, arousal levels should be reduced to help weaken the negative association that tinnitus might have with noisy places for the patient (McKenna, 2004). Reducing autonomic arousal could be achieved through relaxation exercises or cognitive therapy wherein appraisals of tinnitus being threatening are challenged and altered so that the experience is thought of as non-aversive. Decreasing autonomic arousal during, or before entering, feared situations would thus remove the potential for a person to experience relief/benefit from either avoiding or fleeing the situation, and consequently enable them to remain in such contexts. 


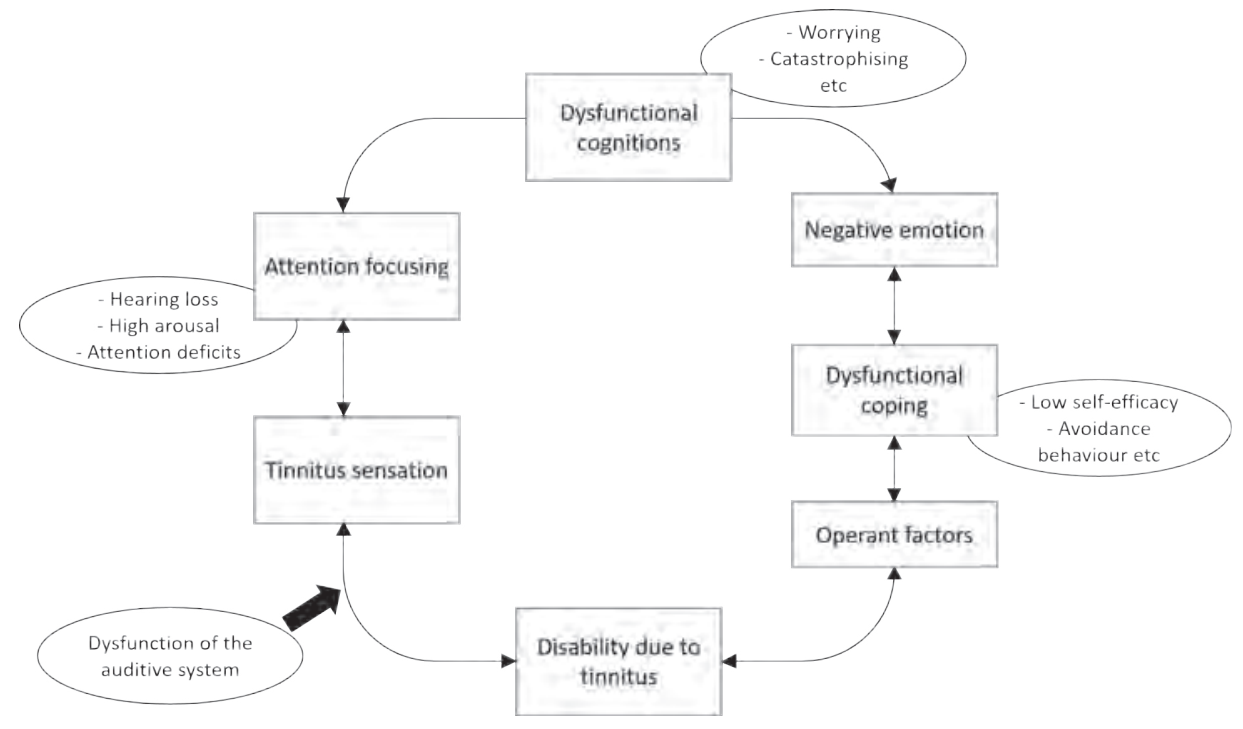

Note: reproduced with permission from Kroner-Herwig et al. (2003; p. 382).

There are however some limitations of the habituation model as it is applied to tinnitus. For example, the model inherently assumes that the characteristics of tinnitus (e.g., perceived tone, volume, frequency and context in which it is experienced) are stable for most people with tinnitus, since most people "habituate" to it. However, this assumption seems problematic as the dynamic characteristics of tinnitus are well-known (Cederroth et al., 2019), and given that any changes in which or how tinnitus is experienced could reasonably be expected to warrant consideration of changed conditions as a "new" presentation of the stimulus, requiring attentional resource allocation. Furthermore, the model does not indicate whether tinnitus distress could occur as a result of sensitization (that is, an increase in response to a repeated stimulus), or dishabituation (the presentation of a "new" stimulus resulting in an increase in response to the original stimulus to which then response has been habituated). If, for example, in cases of disabling tinnitus, the tinnitus and the conditions under which it is experienced are constant, then sensitization to the percept might be part of the explanation for tinnitus distress. However, this is also somewhat counter-intuitive to the fundamental idea of habituation as if it was a constant, stable sound, habituation to tinnitus would seem to be the likely outcome. If though the tinnitus is variable, then two possibilities arise: first, that habituation would never occur since habituation is dependent on repeated exposure of the same stimulus and a decreasing response to that same stimulus; and second, that dishabituation (i.e. the presentation of a "new" stimulus resulting in an increase in response to the original stimulus) or sensitization both seem to be plausible explanations for tinnitus distress. Thus, from these possibilities it is not clear what the most likely explanation for disabling tinnitus might be. A further issue relates to the 
bi-directional arrow between "tinnitus sensation" and "disability due to tinnitus" which implies that hearing tinnitus itself is enough to cause disability. Especially given the presence of cognitive factors in the model, it seems inherently inconsistent to think that perception, without some form of evaluation or belief about the tinnitus, leads to disability. Further to these limitations, there is scant empirical evidence in support of the habituation model overall (Baguley, Andersson, et al., 2013).

\section{A neurophysiological model of tinnitus}

Jastreboff expanded the work of Hallam in a neurophysiological model (see Figure 2), based predominantly on animal research, focussing on tinnitus generation and perception rather than tinnitus-related disability per se. Jastreboff proposed that the association between tinnitus and an aversive emotional state emerges through classical conditioning mechanisms (Jastreboff, 1990; Jastreboff et al., 1988). Classical (or Pavlovian) conditioning refers to the learning of a relationship between two stimuli, a neutral one (conditioned stimulus) and a biologically relevant one (unconditioned stimulus) (Pavlov, 1927). After pairing of the stimuli, subsequent presentation of the neutral one activates the representation of the biologically relevant stimulus (i.e. it elicits a conditioned response) in the absence of the biologically relevant, unconditioned stimulus. According to Jastreboff, successful habituation to tinnitus is expedited through counselling and sound therapy (a combination subsequently labelled as Tinnitus Retraining Therapy; TRT) (Jastreboff \& Hazell, 1993; Jastreboff \& Hazell, 2004). The logic being that to counsel patients according to the TRT protocol is to effectively inform them that there is nothing to worry about or fear from tinnitus itself thereby helping them reduce their emotional response to tinnitus and subsequently decreasing the likelihood that emotional distress is associated with or follows the perception of tinnitus. Additionally, a sound-masking device is used to produce a neutral sound at a volume close to, but below, the perceived loudness of the tinnitus, which according to the model, facilitates habituation by at least partially concealing any novel qualities in the tinnitus, thus making it appear more constant/similar/stable and not worthy of conscious attention. 
Figure 2. Neurophysiological Model of Tinnitus

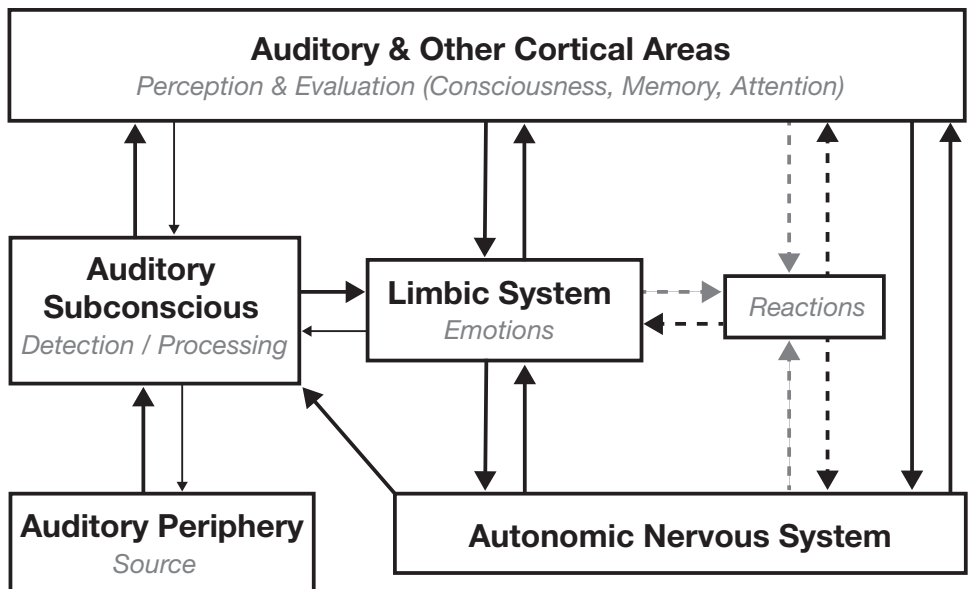

Note: reproduced with permission from Jastreboff (2011; p. 579)

Several theoretical and empirical limitations exist in relation to Jastreboff's model. Firstly, the model does not clearly specify the unconditioned stimulus, conditioned stimulus, conditioned and unconditioned responses (Baguley, Andersson, et al., 2013). That is, for example, it is not clear if tinnitus is supposed to be the neutral stimulus which is paired with an unconditioned stimulus thus ultimately becoming the conditioned stimulus. Second, and assuming that tinnitus has become the conditioned stimulus and an aversive emotional arousal is the conditioned response, the use of a masking device appears contrary to current understandings of extinguishing learned responses, i.e. through exposure therapy (Craske, Hermans, \& Vervliet, 2018). That is, the use of a masking device can be conceptualised as an avoidance and/or safety behaviour which is usually considered counterproductive in the therapeutic context. Within tinnitus research Hesser and Andersson (2009) have demonstrated that avoidance behaviour mediated the relationship between anxiety sensitivity and tinnitus distress. Similarly, Hesser, Pereswetoff-Morath, and Andersson (2009) found that efforts to control the salience of tinnitus through the use of sounds is associated with increased levels of disability. Further to this, within the context of anxiety research, safety behaviours and avoidance have also been shown to preserve threat expectancies (Lovibond, Mitchell, Minard, Brady, \& Menzies, 2009; van Uijen, Dalmaijer, van den Hout, \& Engelhard, 2018). Lastly, by informing or reassuring people suffering from tinnitus that there is nothing to fear, but then using a device to mask the tinnitus, is inherently contradictory if not also counterproductive (McMurtry, McGrath, \& Chambers, 2006). Tinnitus Retraining Therapy however continues to be widely used as a treatment for tinnitus distress, implying widespread practitioner acceptance, despite having limited evidence for its efficacy (McKenna \& Irwin, 2008; Phillips \& McFerran, 2010). This in turn highlights the needs for the important role clinical guidelines can have in promoting evidence-based practice. 


\section{A cognitive-behavioural model of tinnitus distress}

The cognitive-behavioural (CB) model of tinnitus distress proposed by McKenna et al. (2014) (see Figure 3.) emphasises the key role of cognition and the subsequent effects that specific thoughts about tinnitus have on attentional processes and, to a lesser degree, on the behavioural response to tinnitus sounds. McKenna's approach is based on the dual appraisal model (Lazarus, 1991), which differentiates between primary and secondary appraisals. The primary appraisal of tinnitus concerns the causal attributions ("what is it and where does it come from?"), whereas the secondary appraisal concerns the beliefs about the possibilities to reduce it ("what can I do about it?"). For example, a person might initially think of the tinnitus as a symptom of brain damage, and then, potentially, think that neurosurgery is the only solution available. Once the fight or flight response is activated, the model predicts that increasing physiological arousal subsequently leads to further monitoring for/of tinnitus and selectively attending to it. Beliefs about tinnitus and the capacity to cope with it, underpin the automatic (negative) thoughts and motivate the use of safety behaviours that might be used to better endure the tinnitus. The CB model's main strengths lie in its: empirical foundation; compatibility with cognitive models of insomnia and chronic pain; compatibility with CBT; and, that it can be used to generate testable hypotheses.

The model however has several limitations. First, it does not consider motivational, the social and/or environmental, or personal (resilience) factors that might moderate levels of distress. Second, although McKenna et al. (2014) have cited evidence for each part of the model, the studies referred to were not specifically conducted to test the validity of the model. Furthermore and as McKenna et al. (2014) have acknowledged the evidence for each part of the model is generally limited in terms of quality and depth. This is particularly relevant to tinnitus-related safety behaviours where research on this topic is in its infancy. Thirdly, a study that aimed to evaluate the model found: that tinnitus patients did not identify with or recognise all parts of the model; and, path analyses indicated that more than one version of the model might be applicable for modelling tinnitus distress (Handscomb, 2018). These empirical results, by inference, also suggest that other factors recognisable to patients could be added to the model in future and that multiple models might be required to accommodate the heterogeneity of tinnitus. 
Chapter 1

Figure 3. The Cognitive-Behavioural Model of Tinnitus Distress

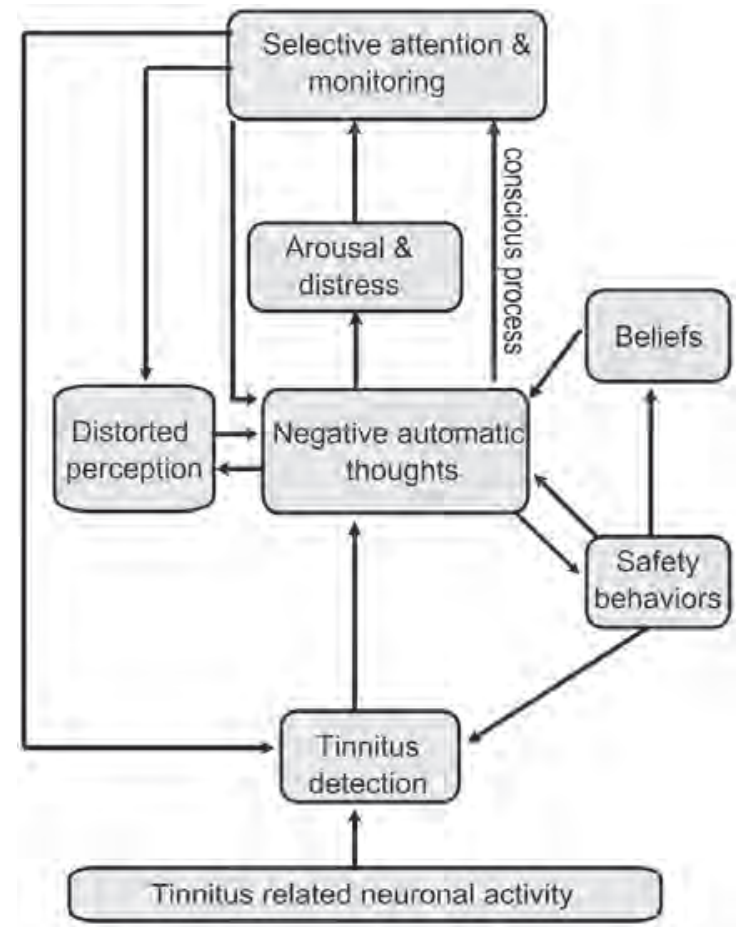

Note: reproduced with permission under CC from McKenna et al. (2014; p. 3)

The main clinical implications of the CB model of tinnitus are that cognitive processes involved in the appraisal of tinnitus should be the target of any treatment. To date there is some evidence that cognitive behavioural therapy (Hesser et al., 2011) and mindfulness based cognitive therapy (McKenna, Marks, Hallsworth, \& Schaette, 2017) are effective in reducing tinnitus-related distress and interference.

\section{Fear avoidance model of tinnitus}

Chronic pain and chronic tinnitus appear to share numerous conceptual similarities, including the perception of a sensation whose source is neither identifiable nor indicative of (further) physical harm (De Ridder et al., 2011; Møller, 1997, 2000). Within the field of chronic pain research, the Fear Avoidance (FA) model (Lethem, Slade, Troup, \& Bentley, 1983; Vlaeyen \& Linton, 2000, 2012) has been highly influential in providing a basis for understanding why some people might experience dysfunctional responses to chronic pain and what therapeutic strategies might be effective. Given the commonalities between chronic pain and tinnitus, tinnitus researchers have adopted the FA model (see Figure 4; Cima et al., 2011; Kleinstäuber et al., 2013) and begun applying it as a basis for treatments to manage tinnitus. 
Figure 4. Fear Avoidance Model of Tinnitus

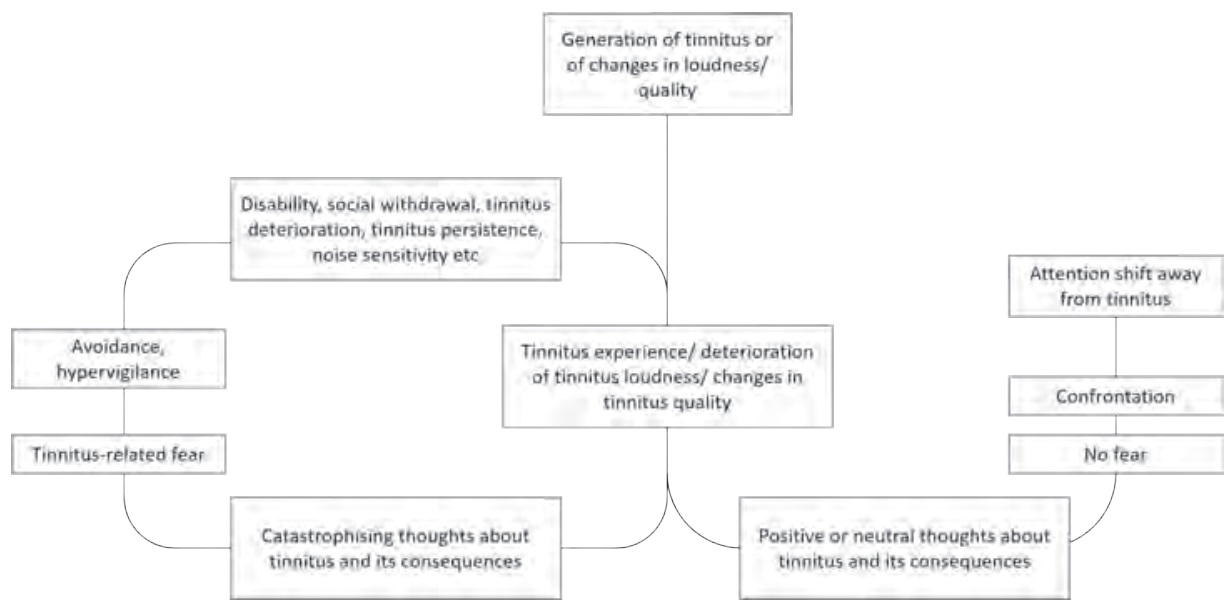

Note: reproduced with permission from Kleinstäuber et al. (2013; p. 86).

The FA model of tinnitus proposes that regardless of the cause of the tinnitus, once it is detected, negative cognitive appraisals (e.g., catastrophic misinterpretations) and aversive emotional reactions (e.g., fear) elicit or can lead to behavioural safety responses. These behavioural responses (e.g., avoidance) might be relieving in the short term but paradoxically can lead to severe impairment in the longer-term. Conversely, neutral or positive evaluation of the tinnitus does not lead to any fear or interference in daily activities.

The FA model of tinnitus distress shares features with both the neurophysiological and the CB model but differs from both in its serial nature and in providing a clear point at which a person perceiving tinnitus might move toward a neutral or negative response. $A$ particular strength of the FA model is that it draws on classical and operant conditioning, which can be used to inform predictions about the acquisition, generalization [i.e., the spread of the effects of conditioning (either classical or operant) to stimuli different from the stimulus present during original conditioning (American Psychological Association, 2020)] and extinction of conditioned responses. While there is a large body of empirical support for the FA model of chronic pain (Markfelder \& Pauli, 2020; Vlaeyen \& Linton, 2012) the evidence for the FA model of tinnitus is small but growing (Cima et al., 2017; Kleinstäuber et al., 2013).

The main clinical implication of the FA model of tinnitus is that it is necessary for catastrophic mis-interpretations or beliefs about tinnitus to be altered so that the ensuing cascade of fear and avoidance behaviours is prevented. Exposure-based treatments have been proposed as an effective treatment to achieve this. They have been found to be effective at reducing fear and avoidance in, for example, the treatment of anxiety disorders (Craske et al., 2018; Craske, Treanor, Conway, Zbozinek, \& Vervliet, 2014) and chronic pain (de Jong, Vlaeyen, van Eijsden, Loo, \& Onghena, 2012; Glombiewski et al., 2018; Vlaeyen, de Jong, Geilen, Heuts, \& van Breukelen, 2001). In the context 
of tinnitus, exposure to a feared situation provides an opportunity for patients to test their catastrophic (mis)interpretations and learn that it is nothing necessarily to fear. To illustrate, a person might specifically fear hearing their tinnitus for any or a prolonged amount of time because they believe they could not cope with it and thus do all they can to avoid it. However, by exposing them to their tinnitus, an opportunity arises for their anticipated/expected catastrophe (i.e. not being able to cope with hearing tinnitus) to be shown to be false. Thus a patient is able to create a new or counter belief that while the experience might be unpleasant, they can tolerate their tinnitus and accomplish other tasks. Although exposure has been included in CBT treatments for tinnitus, its efficacy as a stand-alone treatment has not been established. [Note: a randomised controlled trial $(\mathrm{RCT})$ comparing exposure with masking is currently underway; see the Netherlands Trial Register number NL6235.]

As with all models though, the FA model of tinnitus has been criticized. Wideman et al. (2013) have questioned the assumption of the FA model of chronic pain that the fear of pain (and by extension, fear of tinnitus) is analogous to a phobia (also discussed in Crombez, Eccleston, Van Damme, Vlaeyen, \& Karoly, 2012) and thus leads to exposure as the most empirically supported treatment. By extension there is also an inherent assumption that fear is the only or dominant emotional response in disabling tinnitus. Further, from pain research, Wideman and Sullivan (2012) have argued that an accumulation of risk factors, such as higher pain catastrophizing, fear of movement, and depression, is a better predictor of disability than the individual factors included in the FA model. It remains to be seen whether this also applies in the context of tinnitus. If however the FA model of tinnitus and underlying assumptions that fear of tinnitus is analogous to a phobia are valid, then by extension, there exists an absence of evidence regarding the role that generalization of tinnitus-related fear might play in maintaining or exacerbating disability. Lastly, the FA model of tinnitus does not take in to consideration personal motivational factors or the social context in which patients exist. [Note that the FA model of chronic pain does (Claes, Karos, Meulders, Crombez, \& Vlaeyen, 2014), but the FA model of tinnitus and evidence base for it, lags behind.] 


\section{Summary}

Although these four models differ in varying degrees from one another in their main premise and in terminology used, they all identify mechanisms, predominantly of a cognitive or behavioural nature which might account for disabling tinnitus. The models also provide a rationale for therapeutic strategies which might or might not be compatible with each other. For example, the neurophysiological model which predominantly focuses on tinnitus generation and perception, is linked with TRT in which masking devices are used. Masking devices, however, in the context of the CB or FA models of tinnitus, represent a form of avoidance or safety behaviour and thus appear counter-productive to achieving long-term therapeutic benefit.

\section{Assessment and measurement of tinnitus}

Given the differing definitions of tinnitus, the heterogeneous nature of the symptoms, and the diversity of theoretical models proposed to explain the maintenance of disabling tinnitus, it is no surprise that a universally established, standardised procedure for the assessment of tinnitus is lacking. Examination and investigations from medical practitioners (such as an Ear Nose Throat physicians) are usually conducted to exclude the possibility that tinnitus is caused by an identifiable pathology that could be treated. Psychoacoustic measures of tinnitus (that is, pitch, loudness, minimum masking level) are commonly used in assessments but are not always available or reliable (Henry, 2016; Henry \& Meikle, 2000). However since they do not correlate well with tinnitus severity (Hiller \& Goebel, 2006), they might actually prove more useful in the context of patient education by demonstrating (in) stability of the tinnitus percept over time (Department of Health, 2009). See Table 4 for an overview of available assessment procedures and measures that are commonly used.

Although there is considerable variation in assessment procedures, it is common across the (few) published clinical guidelines recommend use of self-report questionnaires to assess tinnitus and its impact on patients by measuring perceived severity, quality of life, depression and anxiety (e.g., Tunkel et al., 2014). There is a large number of questionnaires available [e.g., the Tinnitus Handicap Inventory (Newman et al., 1996), Tinnitus Functional Inventory (Meikle et al., 2012), Tinnitus Questionnaire (Hallam, 2008; Hallam et al., 1988)], in many languages, and measuring a variety of tinnitus-related domains (Hall et al., 2016). However, not all tinnitus-related questionnaires have been developed in a transparent manner or with a clear purpose in mind; for example it is of importance to know whether a questionnaire is suitable as a diagnostic tool or rather as an outcome measure (Fackrell, Hall, Barry, \& Hoare, 2014). Further, some questionnaires such as The Fear of Tinnitus Questionnaire (Cima et al., 2011) have demonstrated their ability to detect changes in patients following treatment, but have not had their psychometric properties closely examined. This specific gap in the literature is one that we address in chapter 4. 
Chapter 1

Table 4. Assessment and Measurement of Tinnitus Summarised From Cima et al. (2019)

\begin{tabular}{|c|c|}
\hline Purpose & Content \\
\hline $\begin{array}{l}\text { Tinnitus history and } \\
\text { clinical examination }\end{array}$ & $\begin{array}{l}\text { 1. Characteristics of tinnitus: onset, modulation, severity (i.e. is it disabling?) } \\
\text { 2. Audiological history, hearing loss, sensitivity to sound, balance problems } \\
\text { 3. Medical history } \\
\text { 4. Comorbidities, medication and drug use } \\
\text { 5. Relevant personal history (e.g. noise exposure; trauma; social support }\end{array}$ \\
\hline Diagnosis & $\begin{array}{l}\text { 1. Physical examination } \\
\text { 2. Ear, nose and throat examination } \\
\text { 3. Pure tone audiometry, speech audiometry and evaluation of the } \\
\text { perceived quality of tinnitus (e. g. loudness, pitch, and minimum } \\
\text { masking estimations), sound tolerance, tympanometry, and } \\
\text { acoustic reflex including auscultation of the ear and carotid artery } \\
\text { in pulsatile tinnitus, as clinically indicated. }\end{array}$ \\
\hline $\begin{array}{l}\text { Specialised assess- } \\
\text { ment if clinically } \\
\text { indicated }\end{array}$ & $\begin{array}{l}\text { 1. Auditory brainstem responses and/or magnetic resonance imag- } \\
\text { ing (MRI) in cases of unilateral tinnitus and/or asymmetric hear- } \\
\text { ing loss consider High-frequency audiometry in cases of tinni- } \\
\text { tus with normal hearing at standard (conversational) frequencies } \\
\text { 2. Further sound tolerance assessment (e. g. loudness discomfort } \\
\text { level) for sound sensitivity grading or hearing } \\
\text { 3. Dental examination, including temporomandibular joint (TMJ), } \\
\text { in a quiet environment for detecting tinnitus modulations in TMJ } \\
\text { dysfunction or bruxism } \\
\text { 4. MRI of the brain in abnormal auditory brainstem response or ab- } \\
\text { normal vestibular evoked myogenic potential aid settings }\end{array}$ \\
\hline $\begin{array}{l}\text { Assessment of } \\
\text { tinnitus disability }\end{array}$ & $\begin{array}{l}\text { Use standardised and validated questionnaires to measure: } \\
\text { 1. Distress } \\
\text { 2. Severity } \\
\text { 3. Quality of life } \\
\text { 4. Depression } \\
\text { 5. Anxiety }\end{array}$ \\
\hline
\end{tabular}

\section{Treatment of disabling tinnitus}

Just as there is no internationally standardised or recognised pathway for tinnitus assessment, the same applies for the treatment of disabling tinnitus. Currently, no cure for tinnitus exists, and given that, treatments aim to minimise, through a variety of methods, any distress and impairment (Baguley, McFerran, et al., 2013). Treatments include education and advice, CBT, relaxation therapy, TRT and sound enrichment using ear-level sound generators or hearing aids (Henry, Dennis, \& Schechter, 2005; Thompson, Hall, Walker, \& Hoare, 2016). In addition, electrical and magnetic neurostimulation, medicines and herbal remedies have been tested. The effects of these treatment options are variable, they have inconclusive outcomes and some have adverse effects (Dobie, 1999; Hoare, Kowalkowski, Kang, \& Hall, 2011; Langguth \& Elgoyhen, 2011; Langguth, Kreuzer, Kleinjung, \& De Ridder, 2013; Martinez-Devesa, Perera, Theodoulou, \& Waddell, 2010; Phillips \& McFerran, 2010). A summary of available, though not necessarily evidence-based, treatments is presented in Table 5. 
The variation in assessment and treatment of tinnitus could partially reflect a lack of, or inconsistency between, clinical guidelines for tinnitus and an absence of high-quality evidence for the efficacy of treatments. We will address the issue of tinnitus assessment and treatment in clinical guidelines in Chapter 2.

To date, the best supported and evidence-based treatment when using tinnitus-related distress and interference as the outcome measures of treatment efficacy appears to be CBT (G. Andersson \& Lyttkens, 1999; Cima, Andersson, Schmidt, \& Henry, 2014; Martinez-Devesa et al., 2010). This might be because psychological factors mediate or better account for levels of distress and predicting treatment outcome rather than variations in psychoacoustic properties of tinnitus. That said, CBT does not appear to affect the perceived loudness of tinnitus (Martinez-Devesa et al., 2010) and thus, if one was to judge the best treatment for tinnitus using the outcome of perceived loudness, one might reach a different conclusion.

Table 4. Summary of Available Tinnitus Treatments

\begin{tabular}{|c|c|c|}
\hline Treatment & Content & Empirical support \\
\hline Education & - Information about tinnitus & + \\
\hline $\begin{array}{l}\text { Hearing loss treatments for peo- } \\
\text { ple with hearing loss and tinnitus }\end{array}$ & $\begin{array}{l}\text { - Cochlear implants } \\
\text { - Hearing aids }\end{array}$ & $?$ \\
\hline Psychological & $\begin{array}{l}\text { - Cognitive behavioural therapy } \\
\text { - Mindfulness based cognitive therapy } \\
\text { - Exposure therapy (as a stand-alone } \\
\text { treatment) }\end{array}$ & $\begin{array}{l}++ \\
++ \\
?\end{array}$ \\
\hline Pharmaceutical & $\begin{array}{l}\text { - Anti-arrhythmic } \\
\text { - Anticonvulsants } \\
\text { - Anxiolytics } \\
\text { - Glutamate receptor antagonists } \\
\text { - Antidepressants } \\
\text { - Muscle relaxants }\end{array}$ & $\begin{array}{l}- \\
- \\
- \\
- \\
-\end{array}$ \\
\hline Neurostimulation & $\begin{array}{l}\text { - Transcranial direct stimulation } \\
\text { - Transcranial alternating current stimulation } \\
\text { - Repetitive transcranial nerve stimulation } \\
\text { - Vagus nerve stimulation } \\
\text { - Acoustic coordinated reset (CRß) } \\
\text { neuromodulation } \\
\text { - Invasive deep brain stimulation }\end{array}$ & $\begin{array}{l}? \\
? \\
- \\
? \\
? \\
?\end{array}$ \\
\hline Sound therapy & $\begin{array}{l}\text { - Tinnitus masking therapy } \\
\text { - Neuromonics } \\
\text { - Notched music stimulation }\end{array}$ & $\begin{array}{l}? \\
? \\
?\end{array}$ \\
\hline
\end{tabular}


Chapter 1

Table 4. Continued.

\begin{tabular}{lll}
\hline Treatment & Content & Empirical support \\
\hline Combination & $\cdot$ Tinnitus Retraining Therapy & $?$ \\
\hline Alternative therapies & $\cdot$ Dietary supplements & - \\
& $\cdot$ Ginkgo biloba & - \\
& $\cdot$ Melatonin & - \\
& $\cdot$ Acupuncture & $?$ \\
\hline
\end{tabular}

Note: Table generated from information presented in Cima et al. (2019) and Hoare et al. (2011); levels of evidence in the following key refer to the Oxford Centre of Evidence-based medicine criteria. Key: + refers to levels of evidence $2 \mathrm{~b}, 2 \mathrm{c}, 3 \mathrm{a}$ for the use of the treatment; ++ refers to levels of evidence 1a, 1b, 2a for the use of the treatment; ? refers to little or no evidence for or against the use of the treatment; - refers to levels of evidence $2 b, 2 c, 3 a$ against the use of the treatment; -- refers to levels of evidence $1 \mathrm{a}, 1 \mathrm{~b}, 2 \mathrm{a}$ against the use of the treatment.

\section{Cognitive Behaviour Therapy for tinnitus}

Cognitive and behavioural therapies have been applied to treating tinnitus-related distress in RCTs since the mid 1980's (Jakes, Hallam, Rachman, \& Hinchcliffe, 1986). Specific treatment components can include: education about tinnitus and hearing; cognitive restructuring (i.e., identifying and modifying dysfunctional thoughts about tinnitus); attention switching exercises; mindfulness strategies; increasing acceptance of tinnitus; exposure and response prevention; behavioural activation; stress management; modification of lifestyle factors; and applied and/or progressive muscle relaxation (Thompson et al., 2016). Treatments described or labelled as 'CBT' however cannot be assumed to be equivalent entities. Even if CBT treatments appear to comprise the same components, they are likely to vary in at least one of the following:

1. which specific CBT treatment elements (or combinations) are used;

2. mode of delivery (e.g., face-to-face, mediated via telephone, Internet);

3. frequency of session (e.g., daily, weekly, fortnightly);

4. length of session;

5. duration of treatment;

6. who delivers the CBT (e.g., psychologist, social worker, nurse, computer program);

7. the setting in which CBT is delivered (e.g., hospital, health centre, private clinic; online); and,

8. whether the CBT is delivered in a group or individually.

This variety results in clinical and statistical heterogeneity between CBT treatments that can in turn make synthesising existing research in systematic reviews and metaanalyses complex (Hesser et al., 2011). Despite this heterogeneity, conclusions from systematic reviews of the efficacy of CBT for tinnitus have been consistent. They typically report that CBT: reduces the distress and interference associated with tinnitus; appears to have some small beneficial effect on co-morbid depression and anxiety; but does not change the qualitative characteristics the tinnitus itself (Cima et al., 2014; Martinez-Devesa et al., 2010). 
The previous Cochrane systematic review of CBT for tinnitus is now nearly 10 years old (Martinez-Devesa et al., 2010) and since then, new RCTs have been conducted and both methodological advances in conducting RCTs and systematic reviews have emerged. These advances could ultimately affect which studies are selected for inclusion in a review, the results of evidence synthesis, and ultimately conclusions about the efficacy (or otherwise) of CBT for tinnitus. Hence a new review at this time is warranted. We report the findings from the systematic review and meta-analysis in chapter 3.

Since the systematic review by Martinez-Devesa et al. (2010) the largest RCT of CBT conducted to date was completed. It examined the efficacy of specialised stepped-care CBT for tinnitus compared to treatment as usual (Cima et al., 2012). Specialised CBT for tinnitus includes several many components (e.g., behavioural activation, exposure, attention switching exercises, relaxation) and was found to be superior to treatment as usual as delivered in the Netherlands. Specialised CBT for tinnitus, as developed by Cima et al. (2012) has only been tested under RCT conditions. In this dissertation we examine whether it is possible to observe similar effects when implemented under "real world" conditions (Chapter 5). In other words, we tested whether the findings from an efficacy trial (i.e., RCT) can be translated into "routine care".

\section{Summary}

In brief, although there has been much progress made in the assessment and treatment of disabling tinnitus much needs to be done. Consensus on a definition of tinnitus is required to obtain directly comparable, international, epidemiological data on the prevalence and incidence of tinnitus. Questionnaires designed to measure tinnitus have been developed, and tinnitus-relevant domains and study outcomes have been proposed, but psychometric data and clear guidelines about their use in some instances are wanting. Treatment pathways are still fragmented and clinical guidelines remain rare. CBT for tinnitus appears to be the most promising, evidence-based treatment for tinnitus-related distress and interference in daily activities; yet the most recent Cochrane review is nearly 10 years old and used, arguably, an irrelevant measure as its primary outcome. Lastly, a dearth of effectiveness studies limits the degree of confidence that we can have that treatments only tested under RCT conditions will seamlessly transfer into routine care.

\section{Aim and objectives of the dissertation}

In this dissertation we aim to make a novel contribution to the field of tinnitus research by: (a) revealing commonalities and differences between international guidelines for the assessment and treatment of tinnitus; (b) synthesising the existing evidence relating to the efficacy of CBT for tinnitus; (c) examining the psychometric properties of the Fear of Tinnitus Questionnaire; and (d) testing whether it is possible to implement specialised stepped-care CBT based-treatment for tinnitus under "real world" conditions. We address four main research questions. 
Research question 1: What clinical guidelines for the assessment and treatment of tinnitus exist, and what do they recommend? To address this question we conducted a systematic review of existing clinical guidelines for chronic tinnitus. The results of this study are presented in the form of a narrative synthesis in Chapter 2.

Research question 2: Is CBT for tinnitus effective? To answer this question, we conducted a systematic review and meta-analysis of RCTs of CBT for tinnitus. Data from 22 studies were included in meta-analyses to examine the efficacy of CBT when compared to waiting list control, audiological treatment, Tinnitus Retraining Therapy and any other active treatments respectively. The published protocol is included (Appendix 1) and the study is presented in Chapter 3.

Research question 3: What are the psychometric qualities of the Fear of Tinnitus Questionnaire? To answer this question, we assessed the reliability, validity and factor structure of the Fear of Tinnitus Questionnaire. We collected data from an online sample of participants and used exploratory and confirmatory factor analyses to assess these properties. The results of the study are presented in Chapter 4.

Research question 4: Can stepped-care CBT for tinnitus be implemented in every day clinical practice? To answer this question we conducted an uncontrolled, pragmatic study at an audiological rehabilitation clinic in Eindhoven, the Netherlands. Four-hundred and three participants who were seeking help for disabling tinnitus were included in the study. We used multi-level modelling at group level to assess magnitude of change over a 12-month period. The results of this study are presented in Chapter 5.

Finally, in Chapter 6, we provide a summary of the studies conducted in which we discuss the major implications and limitations before suggesting areas for future research. 


\section{References}

American Psychological Association. (2020). APA Dictionary of psychology. Retrieved from https://dictionary.apa.org/stimulus-generalization

Andersson, G. (2003). Tinnitus loudness matchings in relation to annoyance and grading of severity. Auris Nasus Larynx, 30(2), 129-133.

Andersson, G., \& Edvinsson, E. (2008). Mixed feelings about living with tinnitus: A qualitative study. Audiological Medicine, 6(1), 48-54. doi:doi:10.1080/16513860801899355

Andersson, G., \& Lyttkens, L. (1999). A meta-analytic review of psychological treatments for tinnitus. Br J Audiol, 33(4), 201-210.

Andersson, G., \& Westin, V. (2008). Understanding tinnitus distress: Introducing the concepts of moderators and mediators. Int J Audiol, 47(sup2), S106-S111. doi:10.1080/14992020802301670

Asutay, E., \& Västfjäll, D. (2012). Perception of Loudness Is Influenced by Emotion. PLoS ONE, 7(6), e38660. doi:10.1371/journal.pone.0038660

Baguley, D., Andersson, G., McFerran, D., McKenna, L., Baguley, D., Andersson, G., . . . McKenna, L. (2013). Psychological Models of Tinnitus. In Tinnitus: A Multidisciplinary Approach (pp. 102-109): John Wiley \& Sons, Ltd.

Baguley, D., McFerran, D., \& Hall, D. (2013). Tinnitus. Lancet, 382(9904), 1600-1607. doi:10.1016/s0140-6736(13)60142-7

Bauer, C. A., Berry, J. L., \& Brozoski, T. J. (2017). The effect of tinnitus retraining therapy on chronic tinnitus: A controlled trial. Laryngoscope investigative otolaryngology, 2(4), 166-177. doi:10.1002/lio2.76

Beck, A. T., Epstein, N., Brown, G., \& Steer, R. A. (1988). An inventory for measuring clinical anxiety: psychometric properties. J Consult Clin Psychol, 56(6), 893-897.

Blaesing, L., \& Kroener-Herwig, B. (2012). Self-reported and behavioral sound avoidance in tinnitus and hyperacusis subjects, and association with anxiety ratings. Int $J$ Audiol, 51(8), 611-617. doi:10.3109/14992027.2012.664290

Bouton, M. E. a. (2016). Learning and behavior : a contemporary synthesis (Second edition. ed.): Sunderland.

Cederroth, C. R., Gallus, S., Hall, D. A., Kleinjung, T., Langguth, B., Maruotti, A., . . . Schlee, W. (2019). Editorial: Towards an Understanding of Tinnitus Heterogeneity. Frontiers in Aging Neuroscience, 11(53). doi:10.3389/fnagi.2019.00053

Cima, R. F. F. (2018). Bothersome tinnitus : Cognitive behavioral perspectives. [Störender Tinnitus : Kognitive verhaltensbezogene Perspektiven]. HNO, 66(5), 369-374. doi:10.1007/s00106-018-0502-9 
Cima, R. F. F., Andersson, G., Schmidt, C. J., \& Henry, J. A. (2014). Cognitive-behavioral treatments for tinnitus: a review of the literature. J Am Acad Audiol, 25(1), 29-61. doi:10.3766/jaaa.25.1.4

Cima, R. F. F., Crombez, G., \& Vlaeyen, J. W. (2011). Catastrophizing and fear of tinnitus predict quality of life in patients with chronic tinnitus. Ear Hear, 32(5), 634-641. doi:10.1097/AUD.0b013e31821106dd

Cima, R. F. F., Maes, I. H., Joore, M. A., Scheyen, D. J., El Refaie, A., Baguley, D. M., . . . Vlaeyen, J. W. (2012). Specialised treatment based on cognitive behaviour therapy versus usual care for tinnitus: a randomised controlled trial. Lancet, 379(9830), 19511959. doi:10.1016/s0140-6736(12)60469-3

Cima, R. F. F., Mazurek, B., Haider, H., Kikidis, D., Lapira, A., Norena, A., \& Hoare, D. J. (2019). A multidisciplinary European guideline for tinnitus: diagnostics, assessment, and treatment. HNO, 67(Suppl 1), 10-42. doi:10.1007/s00106-019-0633-7

Cima, R. F. F., van Breukelen, G., \& Vlaeyen, J. W. S. (2017). Tinnitus-related fear: Mediating the effects of a cognitive behavioural specialised tinnitus treatment. Hear Res. doi:10.1016/j.heares.2017.10.003

Claes, N., Karos, K., Meulders, A., Crombez, G., \& Vlaeyen, J. W. S. (2014). Competing goals attenuate avoidance behavior in the context of pain. J Pain, 15(11), 1120-1129. doi:10.1016/j.jpain.2014.08.003

Craske, M. G., Hermans, D., \& Vervliet, B. (2018). State-of-the-art and future directions for extinction as a translational model for fear and anxiety. Philosophical transactions of the Royal Society of London. Series B, Biological sciences, 373(1742), 20170025. doi:10.1098/rstb.2017.0025

Craske, M. G., Treanor, M., Conway, C. C., Zbozinek, T., \& Vervliet, B. (2014). Maximizing exposure therapy: An inhibitory learning approach. Behaviour Research and Therapy, 58(0), 10-23. doi:http://dx.doi.org/10.1016/j.brat.2014.04.006

Crombez, G., Eccleston, C., Van Damme, S., Vlaeyen, J. W., \& Karoly, P. (2012). Fearavoidance model of chronic pain: the next generation. Clin J Pain, 28(6), 475-483. doi:10.1097/AJP.0b013e3182385392

Davis, A., \& El Refaie, A. (2000). Epidemiology of Tinnitus. In R. S. Tyler (Ed.), Handbook of Tinnitus (pp. 1-24). San Diego: Singular thompson Learning.

de Jong, J. R., Vlaeyen, J. W. S., van Eijsden, M., Loo, C., \& Onghena, P. (2012). Reduction of pain-related fear and increased function and participation in workrelated upper extremity pain (WRUEP): Effects of exposure in vivo. Pain, 153(10), 2109-2118. doi:http://dx.doi.org/10.1016/j.pain.2012.07.001

De Paepe, A. L., Williams, A. C. C., \& Crombez, G. (2019). Habituation to pain: a motivational-ethological perspective. Pain. doi:10.1097/j.pain.0000000000001533

De Ridder, D., Elgoyhen, A. B., Romo, R., \& Langguth, B. (2011). Phantom percepts: tinnitus and pain as persisting aversive memory networks. Proc Natl Acad Sci U S A, 108(20), 8075-8080. doi:10.1073/pnas.1018466108 
De Ridder, D., Vanneste, S., Weisz, N., Londero, A., Schlee, W., Elgoyhen, A. B., \& Langguth, B. (2014). An integrative model of auditory phantom perception: tinnitus as a unified percept of interacting separable subnetworks. Neurosci Biobehav Rev, 44, 16-32. doi:10.1016/j.neubiorev.2013.03.021

Department of Health. (2009). Provision of Services for Adults with Tinnitus: A Good Practice Guide Retrieved from London, England:

Dobie, R. A. (1999). A review of randomized clinical trials in tinnitus. Laryngoscope, 109(8), 1202-1211. doi:10.1097/00005537-199908000-00004

Eggermont, J. J., \& Roberts, L. E. (2014). Tinnitus: animal models and findings in humans. Cell and Tissue Research, 361(1), 311-336. doi:10.1007/s00441-014-1992-8

Elgoyhen, A. B., Langguth, B., De Ridder, D., \& Vanneste, S. (2015). Tinnitus: perspectives from human neuroimaging. Nat Rev Neurosci, 16(10), 632-642. doi:10.1038/nrn4003

Fackrell, K., Hall, D. A., Barry, J., \& Hoare, D. J. (2014). Tools for Tinnitus Measurement: Development and Validity of Questionnaires to Assess Handicap and Treatment Effects. In F. Signorelli \& F. Turjman (Eds.), Tinnitus: Causes, Treatment and Short \& Long-Term Health Effects (pp. 13-60). New York: Nova Science Publisher.

Fujii, K., Nagata, C., Nakamura, K., Kawachi, T., Takatsuka, N., Oba, S., \& Shimizu, H. (2011). Prevalence of tinnitus in community-dwelling Japanese adults. J Epidemiol, 21(4), 299-304.

Fuller, T. E., Cima, R. F. F., Langguth, B., Mazurek, B., Vlaeyen, J. W., \& Hoare, D. J. (2020). Cognitive behavioural therapy for tinnitus. Cochrane Database Syst Rev, 1, Cd012614. doi:10.1002/14651858.CD012614.pub2

Glombiewski, J. A., Holzapfel, S., Riecke, J., Vlaeyen, J. W. S., de Jong, J., Lemmer, G., \& Rief, W. (2018). Exposure and CBT for chronic back pain: An RCT on differential efficacy and optimal length of treatment. J Consult Clin Psychol, 86(6), 533-545. doi:10.1037/ccp0000298

Hall, D. A., Haider, H., Szczepek, A. J., Lau, P., Rabau, S., Jones-Diette, J., . . Mazurek, B. (2016). Systematic review of outcome domains and instruments used in clinical trials of tinnitus treatments in adults. Trials, 17, 270. doi:10.1186/s13063-016-1399-9

Hallam, R. S. (2008). Manual of the Tinnitus Questionnaire (revised 2008). London: Polpresa Press.

Hallam, R. S., Jakes, S. C., \& Hinchcliffe, R. (1988). Cognitive variables in tinnitus annoyance. Br J Clin Psychol, 27, 213-222. doi:10.1111/j.2044-8260.1988.tb00778.x

Hallam, R. S., Rachman, S., \& Hinchcliffe, R. (1984). Psychological aspects of tinnitus. In Contributions to medical psychology (Vol. 3, pp. 31-53). Oxford: Pergamon Press.

Handscomb, L. (2018). A systematic evaluation of the cognitive behavioural model of tinnitus distress. (PhD), University of Nottingham, Nottingham, U.K.

Henry, J. A. (2016). "Measurement" of Tinnitus. Otology \& Neurotology, 37(8), e276-e285. doi:10.1097/mao.0000000000001070 
Henry, J. A., Dennis, K. C., \& Schechter, M. A. (2005). General review of tinnitus: prevalence, mechanisms, effects, and management. J Speech Lang Hear Res, 48(5), 1204-1235. doi:10.1044/1092-4388(2005/084)

Henry, J. A., \& Meikle, M. B. (2000). Psychoacoustic measures of tinnitus. J Am Acad Audiol, 11(3), 138-155.

Hesser, H., \& Andersson, G. (2009). The role of anxiety sensitivity and behavioral avoidance in tinnitus disability. Int $J$ Audiol, 48(5), 295-299.

Hesser, H., Pereswetoff-Morath, C. E., \& Andersson, G. (2009). Consequences of controlling background sounds: the effect of experiential avoidance on tinnitus interference. Rehabil Psychol, 54(4), 381-389. doi:10.1037/a0017565

Hesser, H., Weise, C., Westin, V. Z., \& Andersson, G. (2011). A systematic review and meta-analysis of randomized controlled trials of cognitive-behavioral therapy for tinnitus distress. Clin Psychol Rev, 31(4), 545-553. doi:10.1016/j.cpr.2010.12.006

Hiller, W., \& Goebel, G. (2006). Factors influencing tinnitus loudness and annoyance. Arch Otolaryngol Head Neck Surg, 132(12), 1323-1330. doi:10.1001/archotol.132.12.1323

Hoare, D. J., Edmondson-Jones, M., Gander, P. E., \& Hall, D. A. (2014). Agreement and Reliability of Tinnitus Loudness Matching and Pitch Likeness Rating. PLOS ONE, 9(12), e114553. doi:10.1371/journal.pone.0114553

Hoare, D. J., Kowalkowski, V. L., Kang, S., \& Hall, D. A. (2011). Systematic review and meta-analyses of randomized controlled trials examining tinnitus management. The Laryngoscope, 121(7), 1555-1564. doi:10.1002/lary.21825

Jakes, S., Hallam, R. S., Rachman, S., \& Hinchcliffe, R. (1986). The effects of reassurance relaxation training and distraction on chronic tinnitus sufferers. Behav Res Ther, 24(5), 497-507. doi:10.1016/0005-7967(86)90029-X

Jastreboff, P. J. (1990). Phantom auditory perception (tinnitus): mechanisms of generation and perception. Neuroscience Research, 8(4), 221-254. doi:http://dx.doi. org/10.1016/0168-0102(90)90031-9

Jastreboff, P. J. (2011). Tinnitus Retraining Therapy. In A. R. Møller, B. Langguth, D. De Ridder, \& T. Kleinjung (Eds.), Textbook of Tinnitus (pp. 575-596). New York, NY: Springer New York.

Jastreboff, P. J., Brennan, J. F., Coleman, J. K., \& Sasaki, C. T. (1988). Phantom auditory sensation in rats: an animal model for tinnitus. Behav Neurosci, 102(6), 811-822.

Jastreboff, P. J., \& Hazell, J. W. (1993). A neurophysiological approach to tinnitus: clinical implications. Br J Audiol, 27(1), 7-17. doi:10.3109/03005369309077884

Jastreboff, P. J., Hazell, J. W., \& Graham, R. L. (1994). Neurophysiological model of tinnitus: dependence of the minimal masking level on treatment outcome. Hear Res, 80(2), 216-232.

Jastreboff, P. J., \& Hazell, J. W. P. (2004). Tinnitus Retraining Therapy: Implementing the Neurophysiological Model. Cambridge: Cambridge University Press. 
Joo, Y. H., Han, K. D., \& Park, K. H. (2015). Association of Hearing Loss and Tinnitus with Health-Related Quality of Life: The Korea National Health and Nutrition Examination Survey. PLoS ONE, 10(6), e0131247. doi:10.1371/journal.pone.0131247

Kim, H. J., Lee, H. J., An, S. Y., Sim, S., Park, B., Kim, S. W., . . Choi, H. G. (2015). Analysis of the prevalence and associated risk factors of tinnitus in adults. PLoS ONE, 10(5), e0127578. doi:10.1371/journal.pone.0127578

Kleinstäuber, M., Jasper, K., Schweda, I., Hiller, W., Andersson, G., \& Weise, C. (2013). The role of fear-avoidance cognitions and behaviors in patients with chronic tinnitus. Cogn Behav Ther, 42(2), 84-99. doi:10.1080/16506073.2012.717301

Kroner-Herwig, B., Frenzel, A., Fritsche, G., Schilkowsky, G., \& Esser, G. (2003). The management of chronic tinnitus: comparison of an outpatient cognitive-behavioral group training to minimal-contact interventions. J Psychosom Res, 54(4), 381-389. doi:S0022399902004002 [pii]

Kuk, F. K., Tyler, R. S., Russell, D., \& Jordan, H. (1990). The psychometric properties of a tinnitus handicap questionnaire. Ear Hear, 11(6), 434-445.

Langguth, B. (2011). A review of tinnitus symptoms beyond 'ringing in the ears': a call to action. Curr Med Res Opin, 27(8), 1635-1643. doi:10.1185/03007995.2011.595781

Langguth, B., \& Elgoyhen, A. B. (2011). Emerging pharmacotherapy of tinnitus. Expert Opin Emerg Drugs, 16(4), 603-606. doi:10.1517/14728214.2011.644273

Langguth, B., Kreuzer, P. M., Kleinjung, T., \& De Ridder, D. (2013). Tinnitus: causes and clinical management. Lancet Neurol, 12(9), 920-930. doi:10.1016/s14744422(13)70160-1

Lazarus, R. S. (1991). Progress on a cognitive-motivational-relational theory of emotion. Am Psychol, 46(8), 819-834. doi:10.1037//0003-066x.46.8.819

Lethem, J., Slade, P. D., Troup, J. D., \& Bentley, G. (1983). Outline of a Fear-Avoidance Model of exaggerated pain perception-I. Behav Res Ther, 21(4), 401-408. doi:10.1016/0005-7967(83)90009-8

Lotto, A., \& Holt, L. (2011). Psychology of auditory perception. Wiley Interdiscip Rev Cogn Sci, 2(5), 479-489. doi:10.1002/wcs.123

Lovibond, P. F., Mitchell, C. J., Minard, E., Brady, A., \& Menzies, R. G. (2009). Safety behaviours preserve threat beliefs: Protection from extinction of human fear conditioning by an avoidance response. Behav Res Ther, 47(8), 716-720. doi:10.1016/j. brat.2009.04.013

Maes, I. H., Cima, R. F. F., Vlaeyen, J. W., Anteunis, L. J., \& Joore, M. A. (2013). Tinnitus: a cost study. Ear Hear, 34(4), 508-514. doi:10.1097/AUD.0b013e31827d113a

Markfelder, T., \& Pauli, P. (2020). Fear of pain and pain intensity: Meta-analysis and systematic review. Psychol Bull, 146(5), 411-450. doi:10.1037/bul0000228 
Martines, F., Bentivegna, D., Di Piazza, F., Martines, E., Sciacca, V., \& Martinciglio, G. (2010). Investigation of Tinnitus Patients in Italy: Clinical and Audiological Characteristics. International Journal of Otolaryngology, 2010. doi:10.1155/2010/265861

Martinez-Devesa, P., Perera, R., Theodoulou, M., \& Waddell, A. (2010). Cognitive behavioural therapy for tinnitus. Cochrane Database Syst Rev(9), CD005233. doi:10.1002/14651858.CD005233.pub3

McKenna, L. (2004). Models of tinnitus suffering and treatment compared and contrasted. Audiological Medicine, 2(1), 41-53. doi:doi:10.1080/16513860410028735

McKenna, L., Handscomb, L., Hoare, D. J., \& Hall, D. A. (2014). A scientific cognitivebehavioral model of tinnitus: novel conceptualizations of tinnitus distress. Front Neurol, 5, 196. doi:10.3389/fneur.2014.00196

McKenna, L., \& Irwin, R. (2008). Sound therapy for tinnitus - sacred cow or idol worship?: An investigation of the evidence. Audiological Medicine, 6(1), 16-24. doi:10.1080/16513860801899389

McKenna, L., Marks, E. M., Hallsworth, C. A., \& Schaette, R. (2017). Mindfulness-Based Cognitive Therapy as a Treatment for Chronic Tinnitus: A Randomized Controlled Trial. Psychother Psychosom, 86(6), 351-361.

McMurtry, C. M., McGrath, P. J., \& Chambers, C. T. (2006). Reassurance can hurt: parental behavior and painful medical procedures. $J$ Pediatr, 148(4), 560-561. doi:10.1016/j.jpeds.2005.10.040

Meikle, M. B., Henry, J. A., Griest, S. E., Stewart, B. J., Abrams, H. B., McArdle, R., . . . Vernon, J. A. (2012). The tinnitus functional index: development of a new clinical measure for chronic, intrusive tinnitus. Ear Hear, 33(2), 153-176. doi:10.1097/ AUD.0b013e31822f67c0

Møller, A. R. (1997). Similarities between chronic pain and tinnitus. Am J Otol, 18(5), 577-585.

Møller, A. R. (2000). Similarities between severe tinnitus and chronic pain. J Am Acad Audiol, 11(3), 115-124.

Newman, C. W., Jacobson, G. P., \& Spitzer, J. B. (1996). Development of the tinnitus handicap inventory. Archives of Otolaryngology-Head \& Neck Surgery, 122(2), 143148. doi:10.1001/archotol.1996.01890140029007

Nondahl, D. M., Cruickshanks, K. J., Huang, G. H., Klein, B. E., Klein, R., Nieto, F. J., \& Tweed, T. S. (2011). Tinnitus and its risk factors in the Beaver Dam offspring study. Int J Audiol, 50(5), 313-320. doi:10.3109/14992027.2010.551220

Noreña, A. J. (2011). An integrative model of tinnitus based on a central gain controlling neural sensitivity. Neurosci Biobehav Rev, 35(5), 1089-1109. doi:10.1016/j. neubiorev.2010.11.003

Pavlov, I. P. (1927). Conditioned reflexes: an investigation of the physiological activity of the cerebral cortex. Oxford, England: Oxford Univ. Press. 
Philippot, P., Nef, F., Clauw, L., de Romree, M., \& Segal, Z. (2012). A randomized controlled trial of mindfulness-based cognitive therapy for treating tinnitus. Clin Psychol Psychother, 19(5), 411-419. doi:10.1002/cpp.756

Phillips, J. S., \& McFerran, D. (2010). Tinnitus Retraining Therapy (TRT) for tinnitus. Cochrane Database Syst Rev(3), CD007330. doi:10.1002/14651858.CD007330.pub2

Ratnayake, S. A., Jayarajan, V., \& Bartlett, J. (2009). Could an underlying hearing loss be a significant factor in the handicap caused by tinnitus? Noise Health, 11(44), 156160. doi:10.4103/1463-1741.53362

Rauschecker, J. P., Leaver, A. M., \& Mühlau, M. (2010). Tuning out the noise: limbic-auditory interactions in tinnitus. Neuron, 66(6), 819-826. doi:10.1016/j. neuron.2010.04.032

Siegel, E. H., \& Stefanucci, J. K. (2011). A little bit louder now: Negative affect increases perceived loudness. Emotion, 11(4), 1006-1011. doi:10.1037/a0024590

Skinner, B. F. (1938). The Behavior of Organisms. New York:: Appleton-Century-Crofts.

Snyder, J. S., Gregg, M. K., Weintraub, D. M., \& Alain, C. (2012). Attention, awareness, and the perception of auditory scenes. Frontiers in Psychology, 3, 15-15. doi:10.3389/ fpsyg.2012.00015

Stockdale, D., McFerran, D., Brazier, P., Pritchard, C., Kay, T., Dowrick, C., \& Hoare, D. J. (2017). An economic evaluation of the healthcare cost of tinnitus management in the UK. BMC Health Serv Res, 17(1), 577. doi:10.1186/s12913-017-2527-2

Thompson, D. M., Hall, D. A., Walker, D. M., \& Hoare, D. J. (2016). Psychological Therapy for People with Tinnitus: A Scoping Review of Treatment Components. Ear Hear. doi:10.1097/aud.0000000000000363

Tunkel, D. E., Bauer, C. A., Sun, G. H., Rosenfeld, R. M., Chandrasekhar, S. S., Cunningham, E. R., . . . Whamond, E. J. (2014). Clinical Practice Guideline: Tinnitus. Otolaryngol Head Neck Surg, 151(2 suppl), S1-S40. doi:10.1177/0194599814545325

Tyler, R. S., \& Baker, L. J. (1983). Difficulties experienced by tinnitus sufferers. J Speech Hear Disord, 48(2), 150-154. doi:10.1044/jshd.4802.150

van Uijen, S. L., Dalmaijer, E. S., van den Hout, M. A., \& Engelhard, I. M. (2018). Do safety behaviors preserve threat expectancy? Journal of Experimental Psychopathology, 9(4), 2043808718804430. doi:10.1177/2043808718804430

Vlaeyen, J. W. S., de Jong, J. R., Geilen, M., Heuts, P. H., \& van Breukelen, G. (2001). Graded exposure in vivo in the treatment of pain-related fear: a replicated singlecase experimental design in four patients with chronic low back pain. Behav Res Ther, 39(2), 151-166.

Vlaeyen, J. W. S., \& Linton, S. J. (2000). Fear-avoidance and its consequences in chronic musculoskeletal pain: a state of the art. Pain, 85(3), 317-332. doi:10.1016/S03043959(99)00242-0 
Vlaeyen, J. W. S., \& Linton, S. J. (2012). Fear-avoidance model of chronic musculoskeletal pain: 12 years on. Pain, 153(6), 1144-1147. doi:10.1016/j.pain.2011.12.009

Wallhäusser-Franke, E., Brade, J., Balkenhol, T., D’Amelio, R., Seegmüller, A., \& Delb, W. (2012). Tinnitus: Distinguishing between Subjectively Perceived Loudness and Tinnitus-Related Distress. PLOS ONE, 7(4), e34583. doi:10.1371/journal. pone.0034583

Wallhäusser-Franke, E., D’Amelio, R., Glauner, A., Delb, W., Servais, J. J., Hörmann, K., \& Repik, I. (2017). Transition from Acute to Chronic Tinnitus: Predictors for the Development of Chronic Distressing Tinnitus. Front Neurol, 8, 605. doi:10.3389/ fneur.2017.00605

Wideman, T. H., Asmundson, G. G., Smeets, R. J., Zautra, A. J., Simmonds, M. J., Sullivan, M. J., . . . Edwards, R. R. (2013). Rethinking the fear avoidance model: toward a multidimensional framework of pain-related disability. Pain, 154(11), 22622265. doi:10.1016/j.pain.2013.06.005

Wideman, T. H., \& Sullivan, M. J. L. (2012). Development of a Cumulative Psychosocial Factor Index for Problematic Recovery Following Work-Related Musculoskeletal Injuries. Physical Therapy, 92(1), 58-68. doi:10.2522/ptj.20110071

World Health Organization. (2001). International classification of functioning, disability and health : ICF: World Health Organization.

Zigmond, A. S., \& Snaith, R. P. (1983). The hospital anxiety and depression scale. Acta Psychiatr Scand, 67(6), 361-370. 
Introduction 
bs:
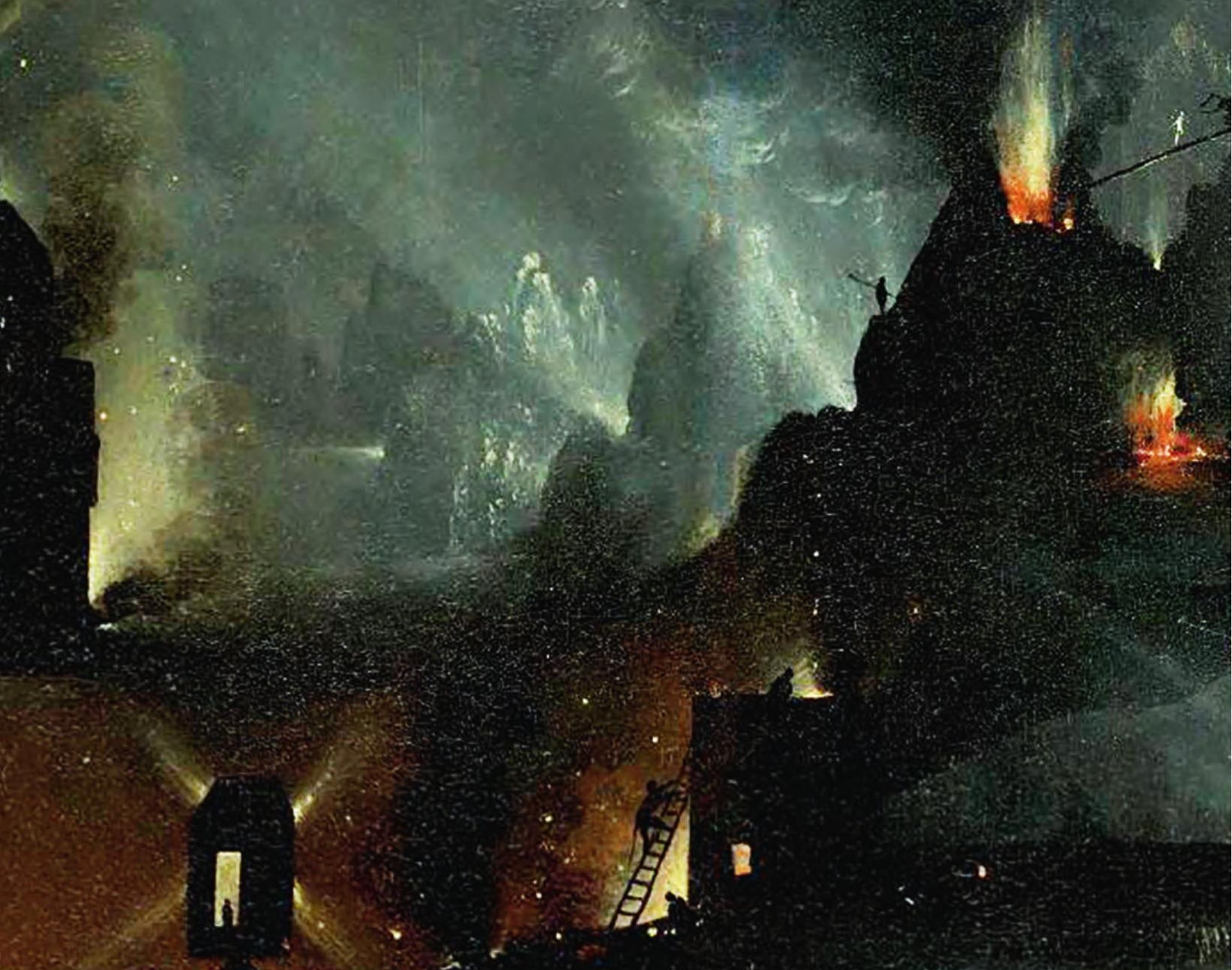

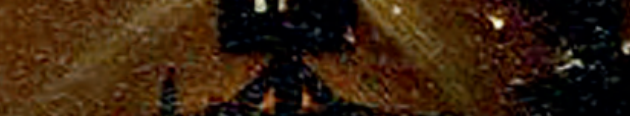

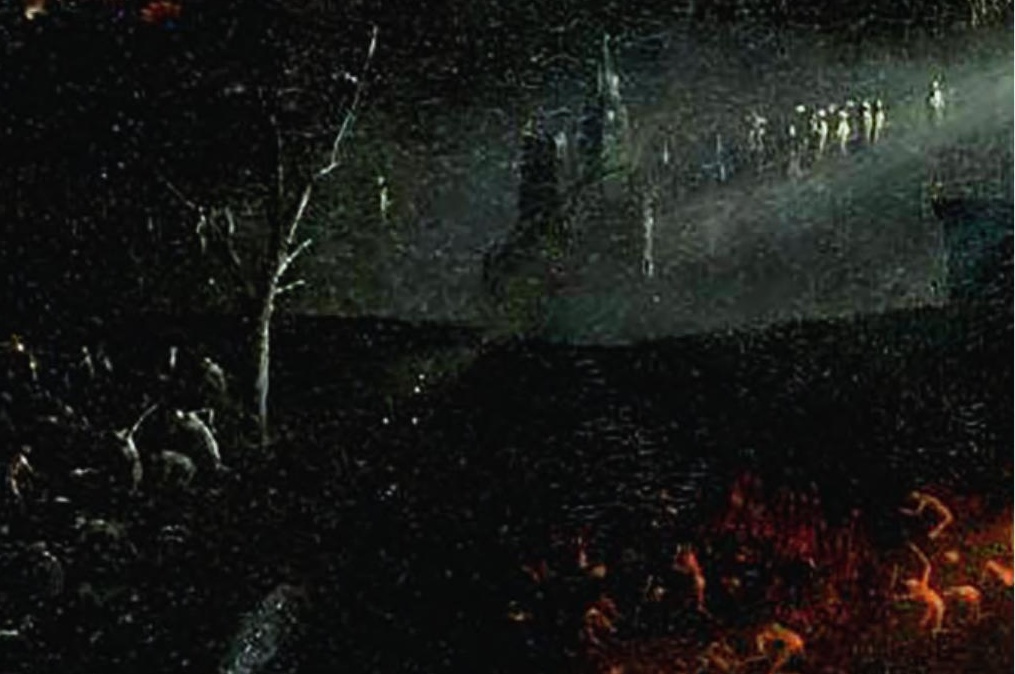




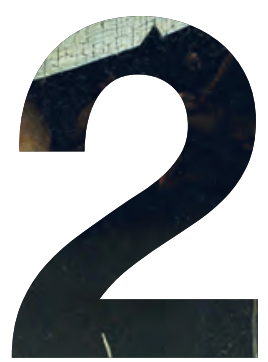

\section{Systematic review of clinical}

\section{guidelines for the assessment and management of chronic tinnitus}

Published as: Fuller TE, Haider HF, Kikidis D, Lapira A, Mazurek B, Norena A, Rabau S, Lardinois R, Cederroth CR, Edvall NK, Brueggemann PG, Rosing SN, Kapandais A, Lungaard D, Hoare DJ and Cima RFF (2017) Different Teams, Same Conclusions? A Systematic Review of Existing Clinical Guidelines for the Assessment and Treatment of Tinnitus in Adults. 


\section{Abstract}

Background Though clinical guidelines for assessment and treatment of chronic subjective tinnitus do exist, a comprehensive review of those guidelines has not been performed. The objective of this review was to identify current clinical guidelines, and compare their recommendations for the assessment and treatment of subjective tinnitus in adults.

Method We systematically searched a range of sources for clinical guidelines (as defined by the Institute of Medicine, United States) for the assessment and/or treatment of subjective tinnitus in adults. No restrictions on language or year of publication were applied to guidelines.

Results Clinical guidelines from Denmark, Germany, Sweden, The Netherlands, and the United States were included in the review. There was a high level of consistency across the guidelines with regard to recommendations for audiometric assessment, physical examination, use of a validated questionnaire(s) to assess tinnitus related distress, and referral to a psychologist when required. Cognitive behavioural treatment for tinnitus related distress, use of hearing aids in instances of hearing loss and recommendations against the use of medicines were consistent across the included guidelines. Differences between the guidelines centred on the use of imaging in assessment procedures and sound therapy as a form of treatment for tinnitus distress respectively.

Conclusion Given the level of commonality across tinnitus guidelines from different countries the development of a European guideline for the assessment and treatment of subjective tinnitus in adults seems feasible. This guideline would have the potential to benefit the large number of clinicians in countries where clinical guidelines do not yet exist, and would support standardisation of treatment for patients across Europe.

Keywords: tinnitus, clinical guidelines, assessment, treatment, systematic review 


\section{Introduction}

Tinnitus is essentially made up of two components, the phantom perception of a sound in the ears or head, and the degree of emotional reaction to that percept. Tinnitus can co-occur with several medical-otological disorders such as presbycusis, though aetiology is unknown for the majority of tinnitus patients (Baguley, McFerran, \& Hall, 2013). In rare cases tinnitus indicates a serious underlying pathology such as vascular troubles, vestibular schwannoma (VS), or otosclerosis (Baguley, Andersson, McFerran, \& McKenna, 2013). In most cases however subjective tinnitus is a benign symptom. In many patients co-morbidities exist such as anxiety, depression, insomnia, and concentration problems, all of which severely impair quality of life (Langguth, Kleinjung, \& Landgrebe, 2011). In 1-3\% of cases tinnitus causes severe health problems, with a wide range of effects on daily life functioning (Davis \& El Refaie, 2000; Fujii et al., 2011; Kim et al., 2015). Evidence corroborates that the aversive psychological reactions, such as cognitive problems, negative emotions, and dysfunctional attentional processes are of main importance in leading to a severe tinnitus condition (Andersson, Juris, Classon, Fredrikson, \& Furmark, 2006; Cima, Crombez, \& Vlaeyen, 2011; Erlandsson \& Hallberg, 2000; Handscomb, Hall, Shorter, \& Hoare, 2017; Kleinstäuber et al., 2013; McKenna, Handscomb, Hoare, \& Hall, 2014)

During the last decades, efforts have been made to better understand tinnitus pathophysiology and provide specialized treatments to patients (Cima et al., 2012; Hoekstra, Wesdorp, \& van Zanten, 2014; Kamalski, Hoekstra, van Zanten, Grolman, \& Rovers, 2010; Langguth, Kreuzer, Kleinjung, \& De Ridder, 2013). A large number of management strategies including various assessment and treatment procedures exist and have evolved but lack empirical support. For example, there is no evidenced treatment or licensed pharmacological therapy to eliminate the tinnitus percept (Langguth \& Elgoyhen, 2012). The Cochrane Library lists 10 completed systematic reviews on different tinnitus treatments, all of which reported small numbers of studies of variable quality (e.g. Martinez-Devesa, Perera, Theodoulou, \& Waddell, 2010). These facts combined makes it difficult for healthcare professionals to decide what is best for which tinnitus patient. This is evidenced by the discrepancy between scientific and clinical perspectives on the management of tinnitus and the actual day-to-day practice in European healthcare settings (Hoare, Gander, Collins, Smith, \& Hall, 2012); tinnitus patient care is fragmented and ad hoc (Hoare et al., 2012; Hoare \& Hall, 2011). To date there has been no overview of the number of existing clinical practice guidelines for tinnitus, the details included, their comparability, or their purpose. Clinical practice guidelines are defined as systematically developed statements to assist practitioner and patient decisions about appropriate health care for specific clinical circumstances (Field \& Lohr, 1990). They have the benefit of simplifying and standardising assessment and treatment options for clinicians and patients. A European Union guideline would extend this benefit to 28 countries. This systematic review aims to identify, review, and examine the clinical guidelines which do exist for tinnitus. The tinnitus assessments (diagnostics and measures), processes, and treatment options recommended by the respective guidelines will be compared and summarized. 


\section{Method}

The aims, the work plan, and the protocol for this systematic review were developed by TINNET Working Group 1, a COST Action BM1306 (2014-2018) to create a panEuropean tinnitus research network [http://tinnet.tinnitusresearch.net/]. This review was registered with $\mathrm{PROSPERO}$, the international register of systematic reviews (protocol number: CRD42016038588) prior to commencing the literature search. The review was exempt from human ethics procedures as there were no human participants and only secondary sources of data (the clinical guidelines) were used.

\section{Eligibility criteria}

Records were considered eligible for inclusion if they fit the definition of a guideline by describing and making recommendations on the assessment, diagnosis, and or treatment of subjective tinnitus for adults (i.e. people aged 16 years or older). Those records were required to identify or describe themselves as guidelines, and be the most recent guideline form the country of origin. No publication date or language restrictions were imposed on the eligibility of the guidelines.

Guidelines were excluded if they were for objective tinnitus, paediatrics, referred only to the triage or referral pathways for assessing and treating tinnitus, or if they were a guide for only one specific type of assessment or treatment procedure for tinnitus.

\section{Literature search}

The literature search for clinical guidelines included the Medline, PubMed, and the Cumulative Index to Nursing and Allied Health Literature (CINAHL), and EMBASE databases. In addition to these the National Guideline Clearinghouse (www.guideline. gov), National Institute for Health and Clinical Excellence (NICE; https://www.nice.org. uk/), Guideline International Network (GIN; http://www.g-i-n.net/), Google, and handsearch of reference lists of any included guidelines was undertaken. International experts were also contacted to ask if they were aware of any guidelines that had not already been identified from the search results. The date that the search for guidelines was first conducted was 2 May 2016 and was undertaken by TEF and HH using "tinnitus" and "guideline" as the two key terms. The final search was conducted on 24 June 2016.

\section{Study selection}

Two reviewers independently screened search results by title and abstract, and then by full text if required. The first 20 pages of search results from Google, and all search results from GIN, NICE, and the National Guideline Clearinghouse were screened. In the event of disagreements, a third reviewer (BM) acted as an arbiter. As an additional check and in line with other systemic review searches using internet search engines, a post-hoc rule of stopping searching after three consecutive pages without new search results was applied. In this case, no new search results were identified after the first eight pages. 


\section{Data extraction}

Data extraction was undertaken using a tailored form that had been pilot tested and was emailed to reviewers in the form of an Excel spreadsheet. A document with guidance on the extraction of information for each of the items was provided to each of the reviewers to improve consistency of data extraction. Data extraction from each guideline was undertaken by at least two reviewers who were native speakers of or fluent in the language in which the guideline was published. Reviewers extracted information from the guidelines regarding items about the: country and year of publication, availability, author details, sponsor/funder involved, scope, target audience, developers and process related to the guideline, recommendations for assessment and treatment procedures, the level of evidence and type of rating system used (e.g. Oxford) related to the recommendations, and items related to the implementation and revision of the guideline.

\section{Data Management}

$\mathrm{HH}$ and TEF were responsible for data management and maintained editorial rights. All identified records were saved into a Microsoft word master file and then saved in pdf-copy.

\section{Quality assessment and risk of bias}

All reviewers of the guidelines also completed the AGREE II tool (Brouwers et al., 2010) to assess the quality of the guidelines. AGREE II is an international tool to assess the quality and reporting of practice guidelines (www.agreetrust.org). It contains 23 items grouped under six guideline domains. Each item is scored on a 1-7 scale where $1=$ 'Strongly disagree' and 7 = 'Strongly agree'. Scores are standardised to provide an overall percentage score. Previous reviews have used a $60 \%$ marker to distinguish high and low quality guidelines (Ruszczynski, Horvath, Dziechciarz, \& Szajewska, 2016; Sanclemente, Acosta, Tamayo, Bonfill, \& Alonso-Coello, 2014).

Details relating to the sources of funding, professional affiliations, and editorial independence of the guideline developers were extracted as indicative of risk of bias.

\section{Data synthesis}

Data extracted by the reviewers were collated and integrated into summary tables and a narrative synthesis describing the similarities and differences between the clinical practice guidelines was completed 


\section{Results}

Five clinical guidelines for tinnitus were ultimately included in this review (see Figure 1 for details of the search and selection process). They were guidelines from Denmark (Jørgensen, Amt, Nemholt, Kristensen, \& Ellesøe, 2007), Germany (The Association of the Scientific Medical Societies, 2015), The Netherlands (Dutch Association for Ear Nose Throat and Head surgery [Nederlandse Vereniging voor Keel - Neus - Oor heel kunde en Heelkunde van het Hoofd-Halsgebied], 2016), Sweden (Idrizbegovic, Kjerulf, \& Team for Diagnostics Hearing Habilitation Children and Youth and Hearing Rehabilitation for Adults, 2011) and United States (Tunkel et al., 2014). Several documents were excluded as by definition not providing a guideline. For example, the Australian audiology clinical practice standards (Audiology Australia, 2013) underwent full-text screening but was not included as it only related to audiological management and had a brief section on tinnitus assessment. The UK Good Practice Guide (Department of Health, 2009) also was excluded as it explicitly states: "This Good Practice Guide to the delivery of services is not, and does not aim to be, an evidence-based guideline for clinical practice with individual patients" (p.5). The Tinnitus Research Initiative (TRI) algorithm (Biesinger et al., 2010), after some debate within the review team, was also excluded because it was judged not to be a "clinical guideline". A list of full text documents considered but excluded is in the file "Chapter 2 Supplementary Information File". 
Figure 1. PRISMA Flowchart Showing the Stages of Guideline Search, Screening and Inclusion
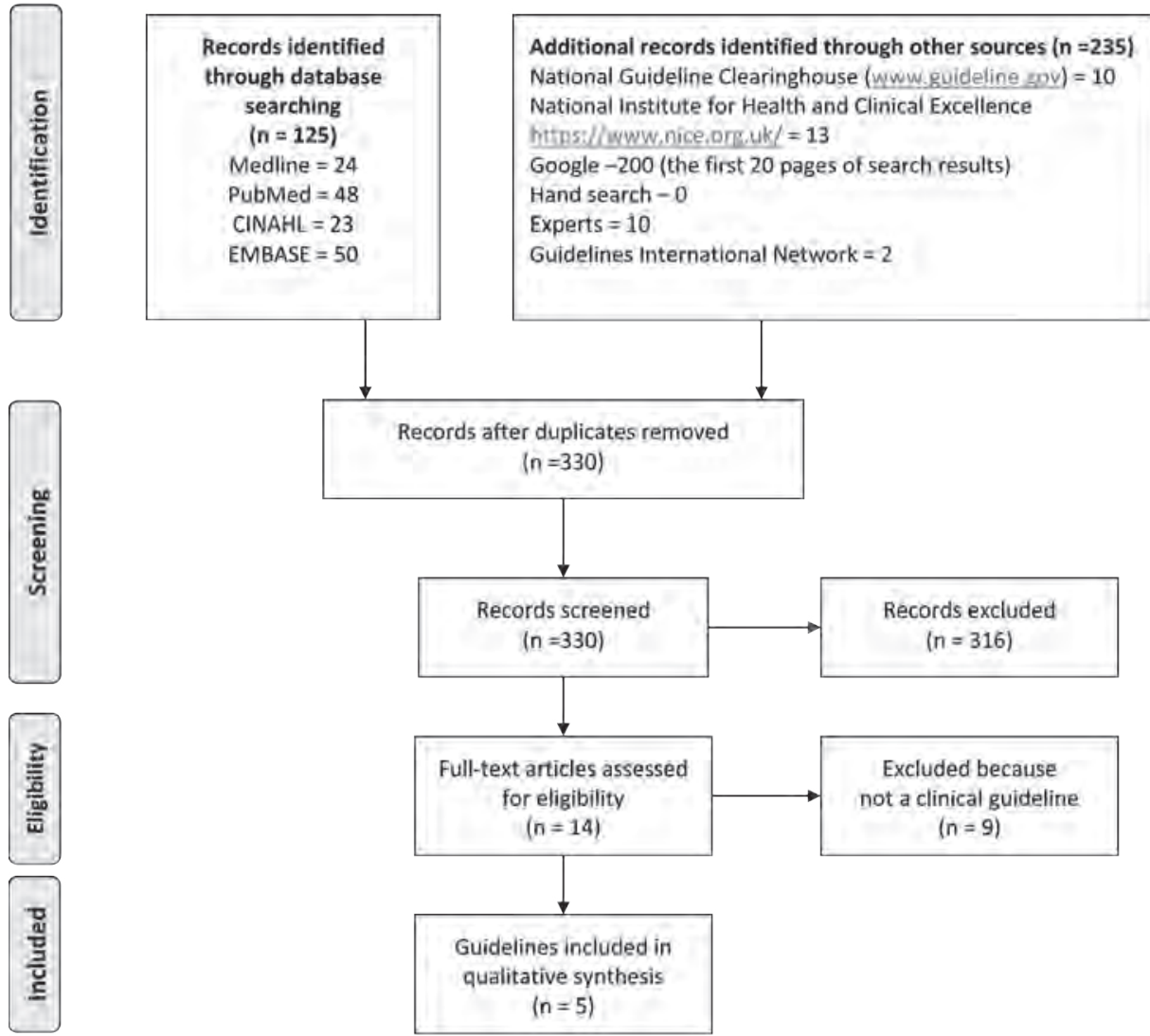

Although there was not a restricting time period for the guidelines, no guidelines older than 10 years were identified. With exception of the Danish guideline (published in 2007) all were developed during the last five years.

\section{Details about development of the clinical guidelines}

Table 1 provides detailed information about the stakeholder involvement, rigour of development, and the editorial independence associated with the respective clinical guidelines.

All the guidelines included information on the professional backgrounds of the participants in the respective development groups and in three out of the five cases (American, Dutch, and German), provided information on how views of funding bodies and competing interests were addressed. Although patient groups and the public were consulted in the development of three guidelines (American, Dutch, and German), the actual expected users of the guidelines were health professionals. 
Details were provided in all guidelines (with the exception of those from Sweden which did not provide methodological information) about how literature was located and used to inform the respective recommendations. That is, details of search strategies using $\mathrm{MeSH}$ and other search terms and databases such as Medline and PsychInfo were included. Tools and criteria used to assess the evidence included the: Oxford Centre for Evidence Based Medicine (U.S. and German guidelines) and American Academy of Paediatrics' (American guideline) evidence criteria respectively, the AMSTAR checklist (Dutch guideline), and the GRADE ranking system of trust in conclusions of the literature (Dutch guideline). American, Danish, Dutch and German guidelines all provided information and referred to the research literature associated with each recommendation as well as describing their methods for reaching consensus on each recommendation. The Dutch, German and U.S. guidelines consider the strengths and limitations of the research literature and were reviewed externally prior to publication. Similarly, those three guidelines also state a year by and/or describe conditions under which they would be revised

\section{Assessment recommendations in the clinical guidelines}

Table 2 compares assessment recommendations between the respective national tinnitus guidelines. All guidelines, except the Danish, recommend a clinical history (anamnesis/targeted history/special tinnitus anamnesis) be taken.

All guidelines describe the need for physical examination by an ENT doctor, although physical examination is not explicitly referred to in the Swedish guideline. The American guideline recommends examination to exclude objective tinnitus, cardiovascular disease and vascular lesions, neurologic diseases, middle or outer ear infection/disease, vertigo, head-neck masses, or other treatable conditions. The German guideline additionally mentions cervical, dental, and temporomandibular joint functional exploration in a silent environment to evaluate tinnitus modulation.

Audiological assessment was recommended in all the included guidelines. The majority refers to audiometry as a general category, but the German guideline provides most detail. For example, it specifies details relating to the assessment of oto-acoustic measurements, brainstem auditory evoked responses, caloric tests, determination of tinnitus loudness and frequency using narrow-band noise and pure tones, residual inhibition, Feldmann masking curves (Feldmann, 1984), and loudness discomfort level. None of the other guidelines included in this review recommend psychoacoustic measurements of tinnitus frequency or intensity.

The German guideline does not refer to specific psychological assessments though the other guidelines do in varying terms. For example, when tinnitus is severe or accompanied by psychological factors, the Swedish guideline recommends psychological assessment while the Danish guideline recommends a structured interview. The American guideline on the other hand recommends that clinicians distinguish between patients with or without bothersome tinnitus for subsequent referral (when necessary) to a psychologist or psychiatrist. 


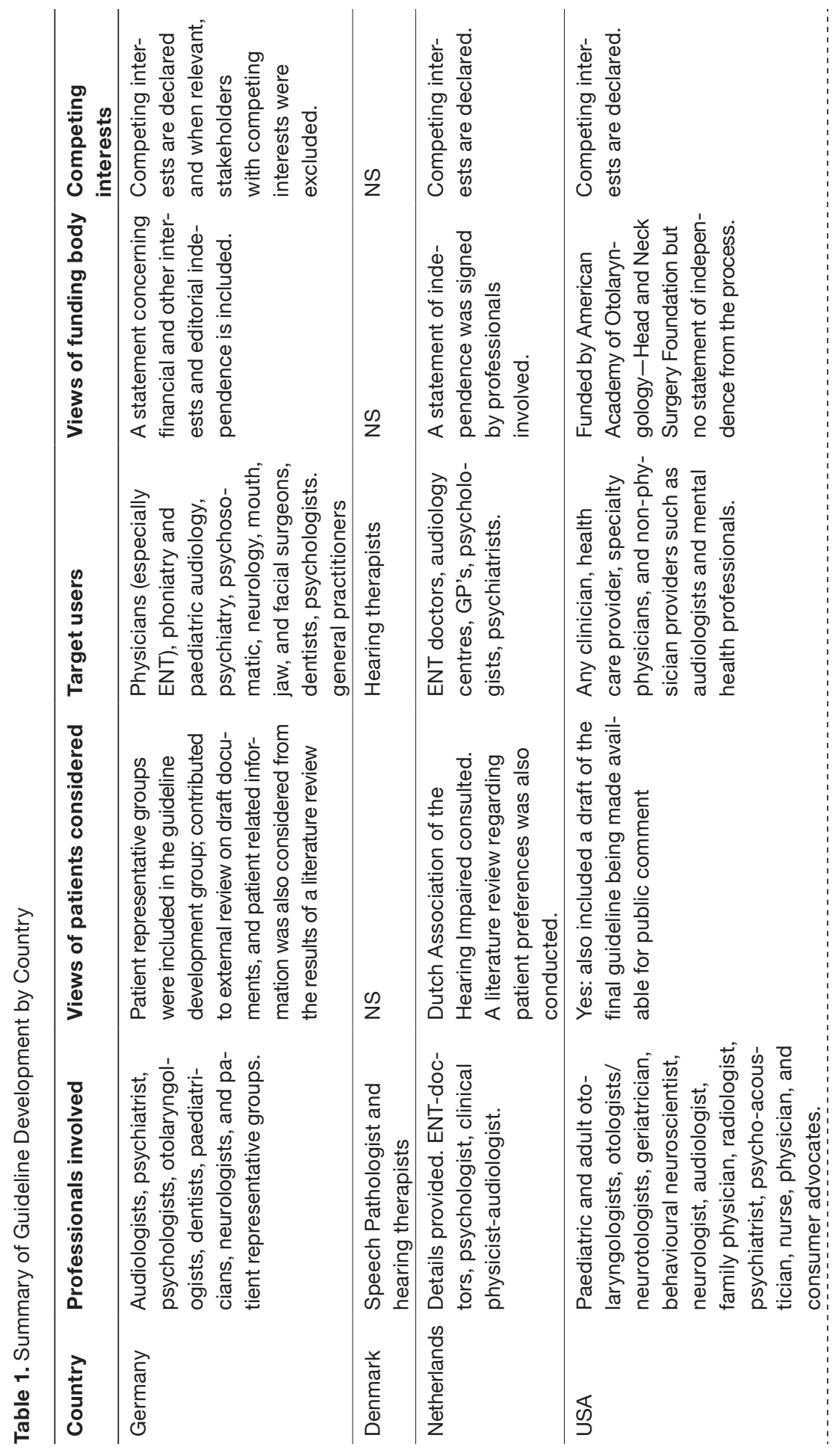




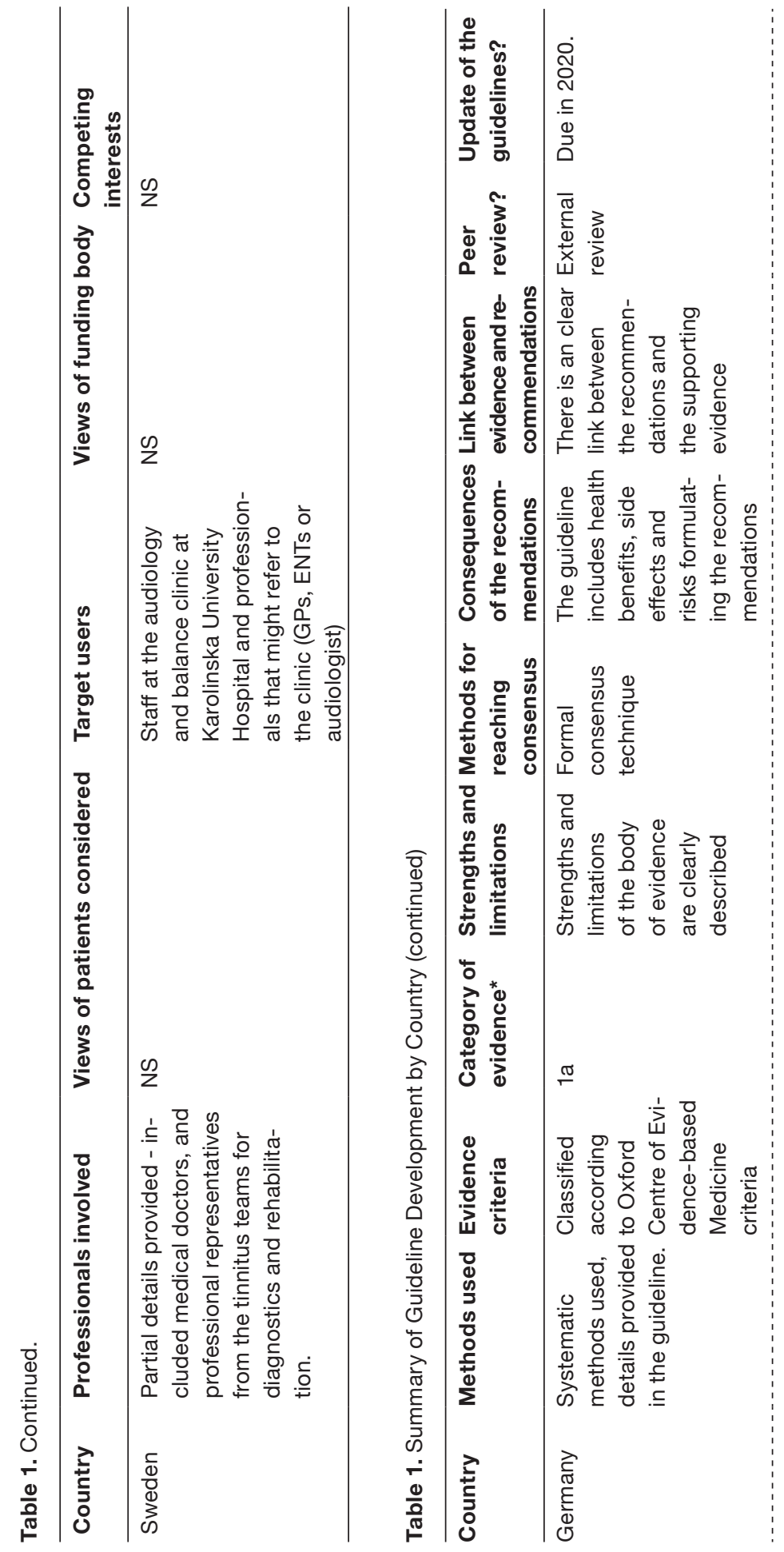




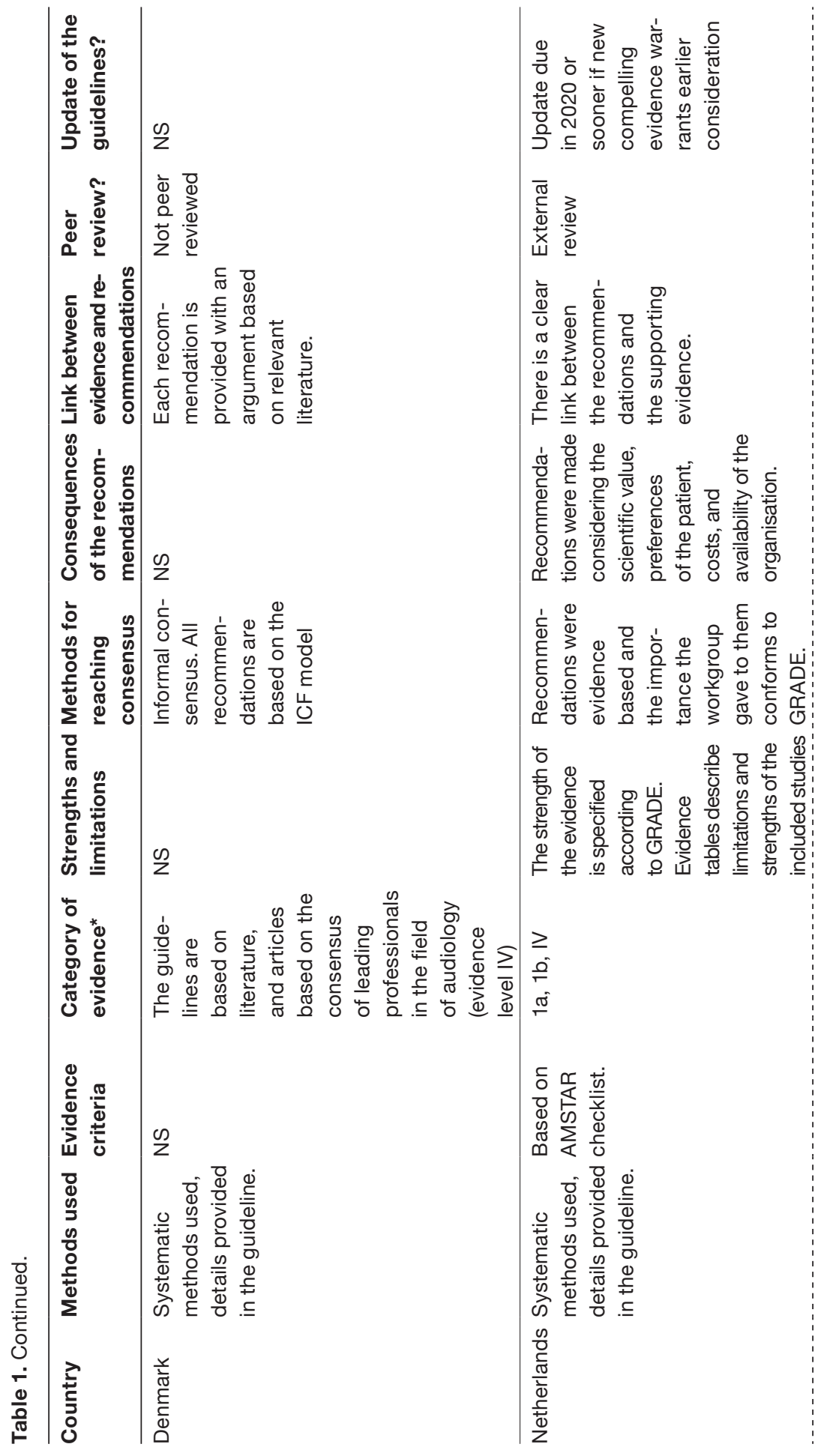




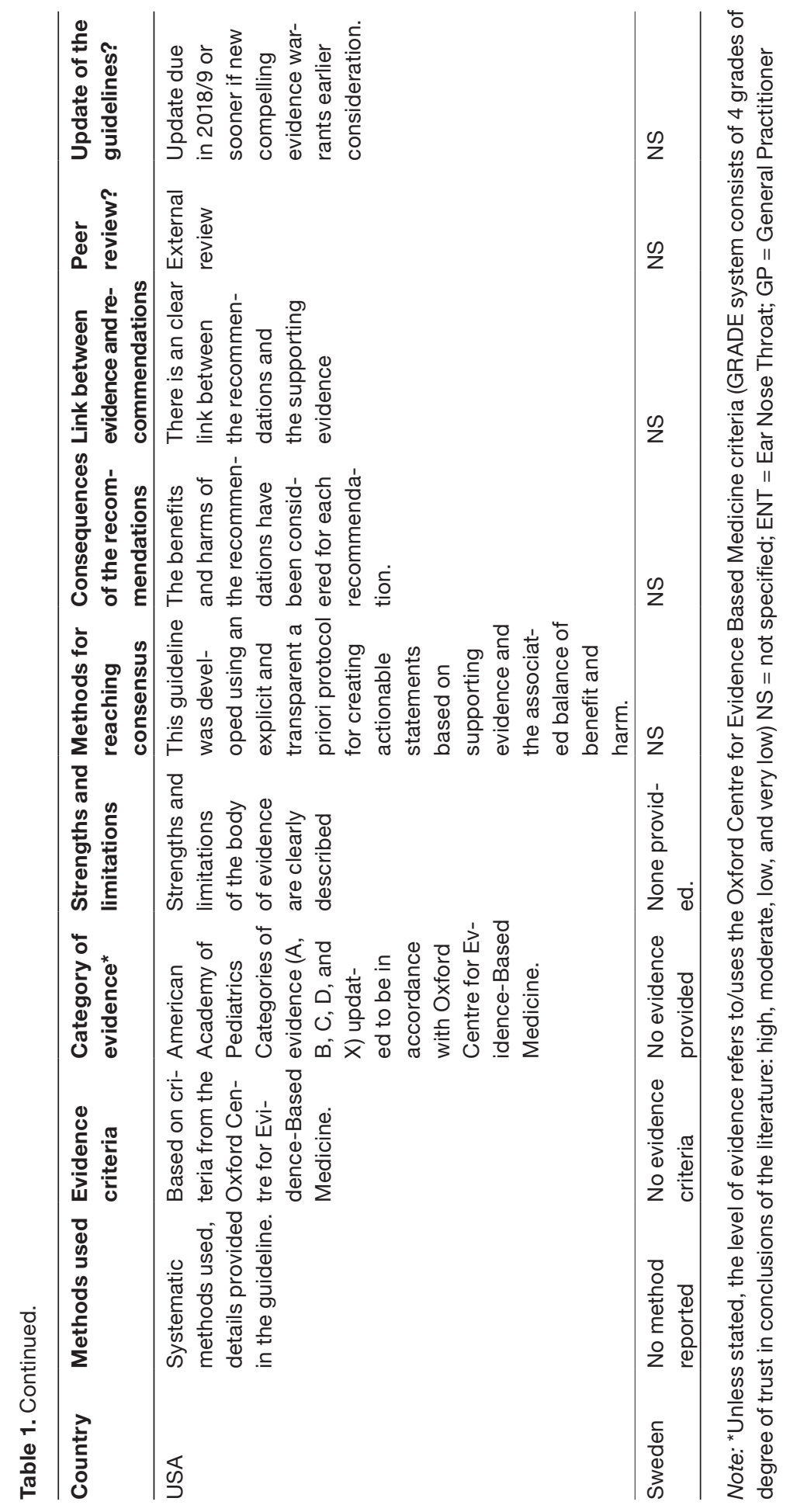




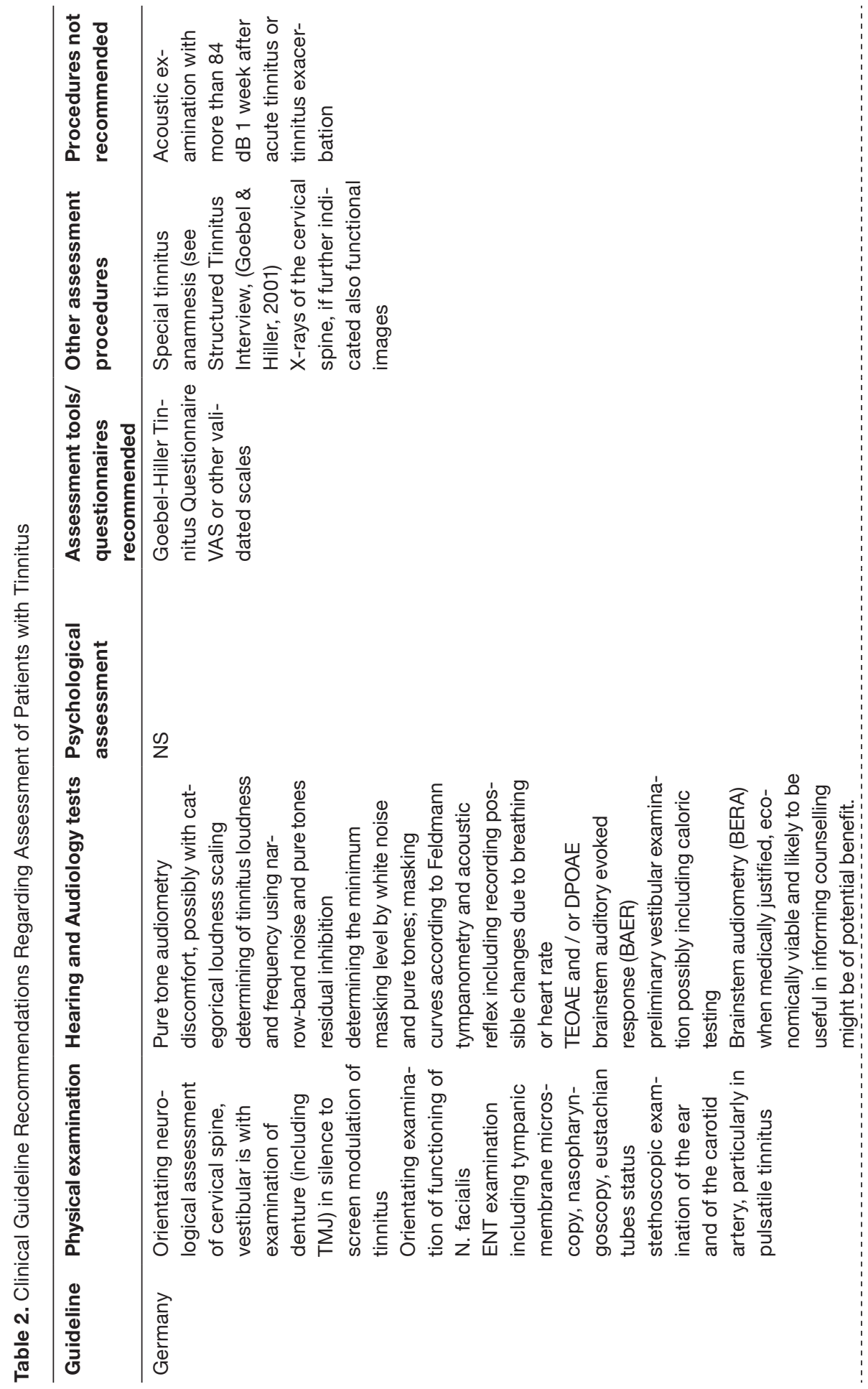


Chapter 2

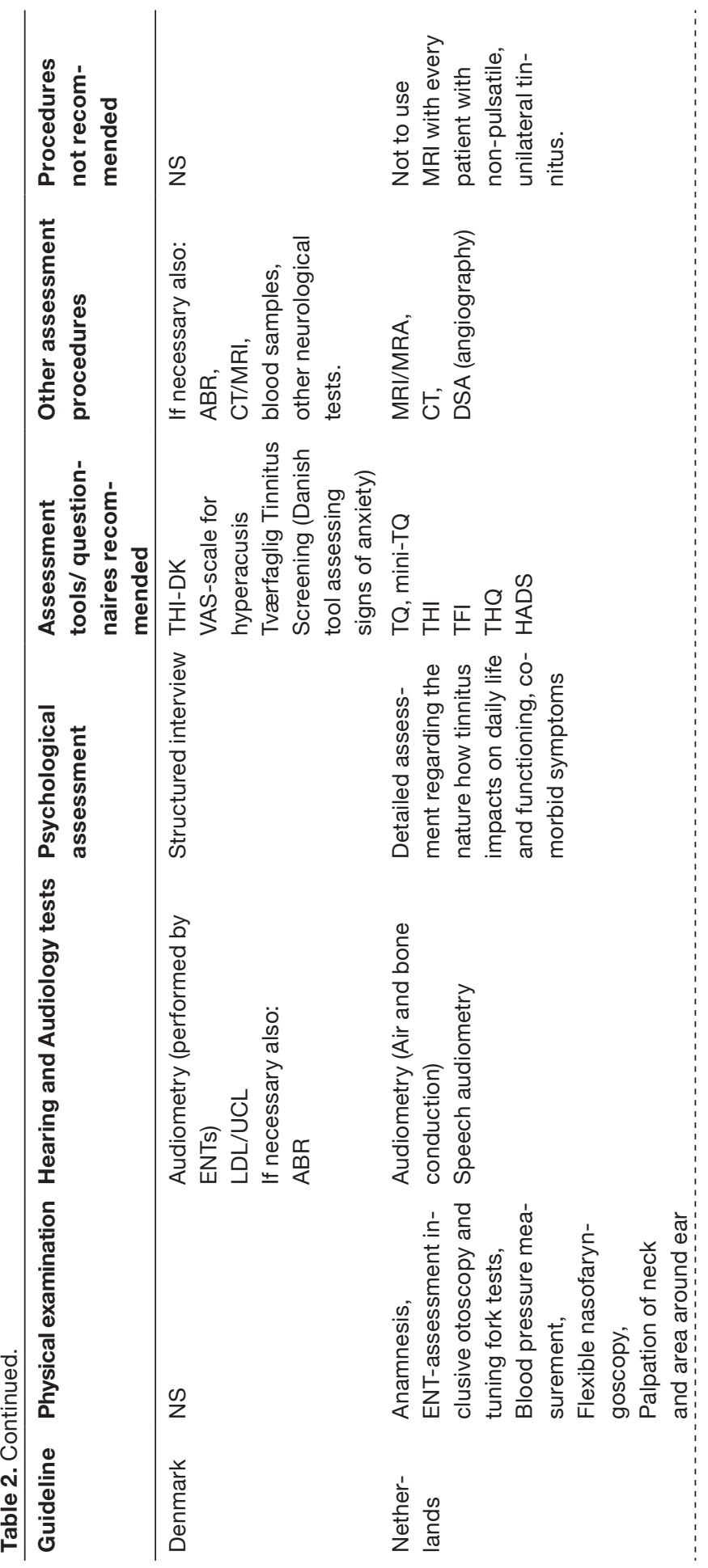




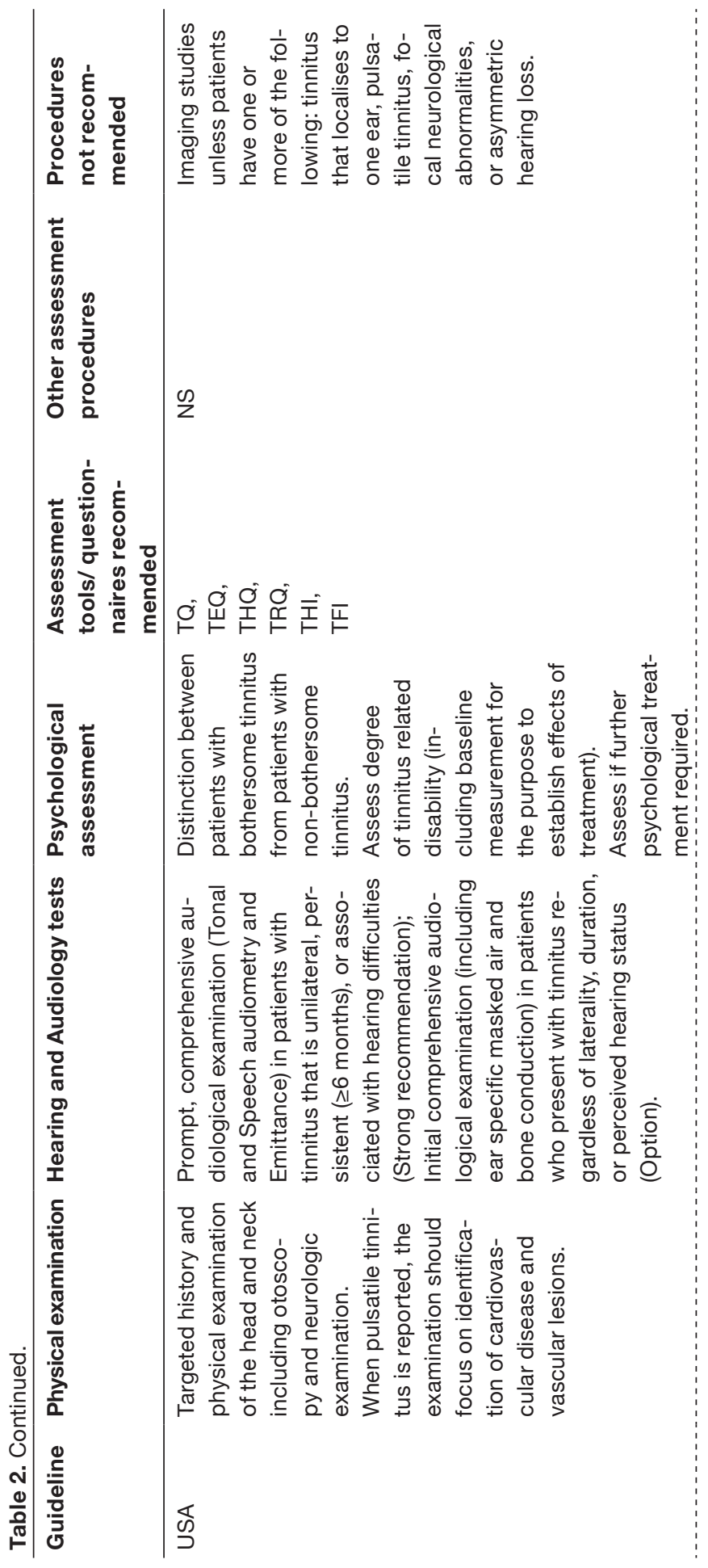




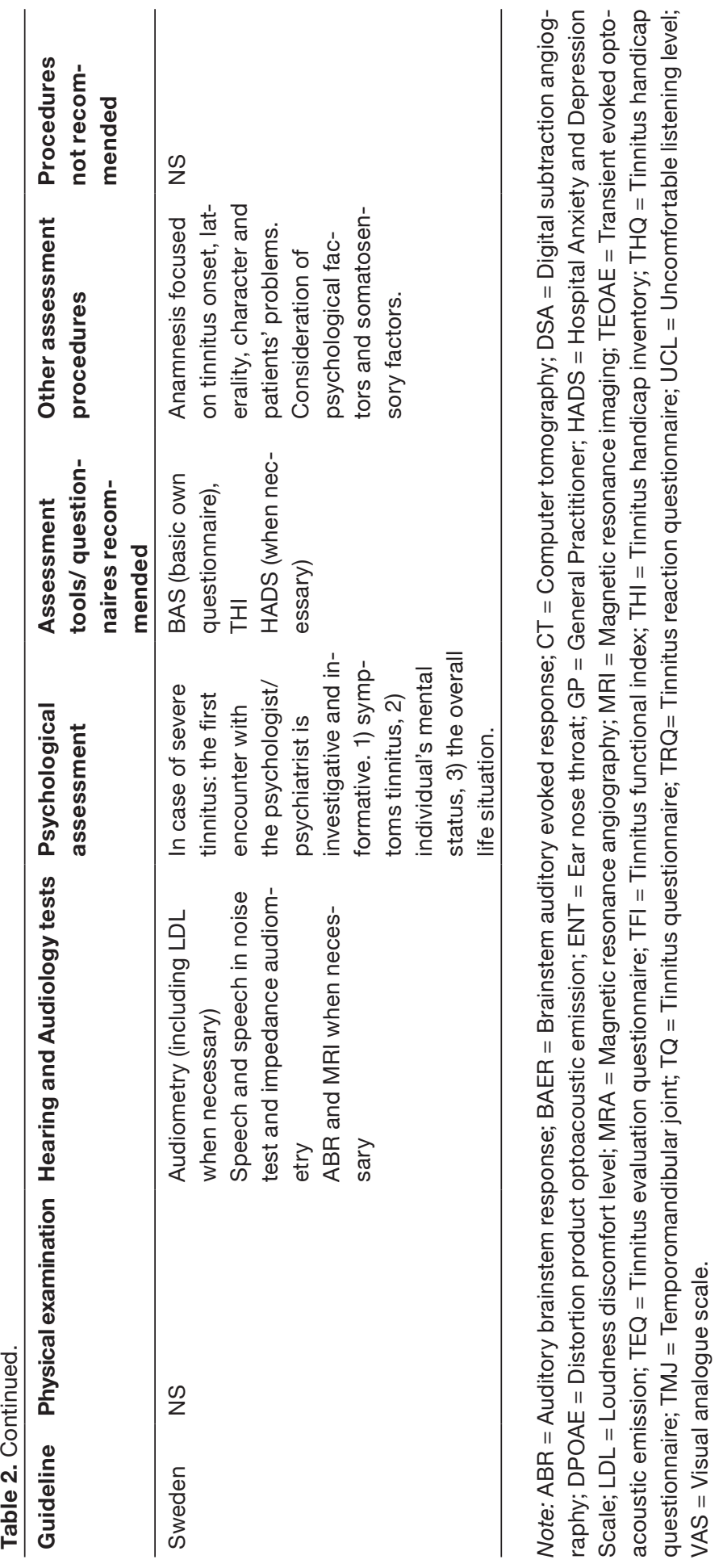


The Tinnitus Handicap Inventory (THI; (Newman, Jacobson, \& Spitzer, 1996; Newman, Sandridge, \& Jacobson, 1998) is the most frequently referred to assessment questionnaire followed by Tinnitus Questionnaire (TQ; (Goebel \& Hiller, 1994). Visual Analogue Scales (VAS; e.g. Germany, Denmark) and the Hospital Anxiety and Depression Scale (HADS; (Zigmond \& Snaith, 1983) e.g. The Netherlands, Sweden) were referred to by at least two guidelines. The American guideline referred to a large number of questionnaires including the: TQ (Goebel \& Hiller, 1994), THI (Newman et al., 1996; Newman et al., 1998), Tinnitus Effects Questionnaire (TEQ; (Hallam, Jakes, \& Hinchcliffe, 1988), Tinnitus Handicap Questionnaire (THQ; (Kuk, Tyler, Russell, \& Jordan, 1990), Tinnitus Reaction Questionnaire (TRQ; (Wilson, Henry, Bowen, \& Haralambous, 1991), and Tinnitus Functional Index (TFl; (Meikle et al., 2012).

Several guidelines make recommendations for or against the use of other assessment related procedures. For example, the German guideline refers to X-rays of the cervical spine. Although three guidelines recommend magnetic resonance imaging (MRI) as an assessment of tinnitus, The American and the Dutch guideline recommend against it, unless patients have one or more of: tinnitus that localizes to one ear, pulsatile tinnitus, focal neurological abnormalities, or asymmetric hearing loss. The German guideline also recommends against acoustic examination using sound pressure levels more than 84 $\mathrm{dB}$ one week after acute tinnitus or tinnitus exacerbation.

\section{Summary of recommendations regarding the assessment of subjective tinnitus}

- Conduct a thorough physical examination to exclude possible (physical) causes of tinnitus (three of five guidelines; not stated in Danish and Swedish)

- Complete a thorough audiological assessment (all guidelines)

- Establish the degree to which a patient experiences subjective tinnitus as bothersome or distressing using a validated and reliable multi-item questionnaire such as the TQ, THI, TFI, or HADS (all guidelines)

- In situations where patients appear to be experiencing a degree of distress or difficulties related to living with tinnitus, consider making a referral for an assessment by a psychologist or psychiatrist (four of five guidelines; not stated in German guideline)

- Variation exist in recommendations regarding the use of imaging studies (e.g. MRI)

\section{Treatment recommendations across the guidelines}

Table 3 compares therapeutic recommendations for the treatment of subjective tinnitus between the respective national tinnitus guidelines; note the Danish guideline is not included in this table as it provides only recommendations regarding assessment procedures. Across the guidelines there is generally a high degree of consistency in the recommendations for or against: the use of medicines (prescribed drugs and herbal supplements); audiological and psychological interventions; and, transcranial magnetic stimulation. Greatest variation occurs in the recommendations concerning the use of therapies involving sound such as Tinnitus Retraining Therapy (TRT). 
There is a consensus that medicines should not be prescribed for the treatment of subjective tinnitus, though some variation in the level of specificity that each guideline has. For example, the German guideline lists specific medicines that should not be prescribed for the treatment of tinnitus. The German and Swedish do however note that medicines such as antidepressants might be prescribed to treat comorbid conditions. Herbal supplements such as Gingko biloba are also specifically recommended against being used in all guidelines except for Sweden which does not make recommendations for or against their use.

The use of hearing aids is recommended by all guidelines but only when clinically meaningful hearing loss is also present in people suffering from tinnitus. The use of a cochlear implant is mentioned in the Dutch and German guidelines and only recommended when there is profound hearing loss or deafness in addition to tinnitus. The Dutch guideline is the only one to provide scores on tinnitus questionnaires (e.g. $T Q, T H I)$ for when such interventions should be considered (e.g. it recommends referral to specialised stepped-care CBT for tinnitus in cases where TQ score is greater than 30 , in combination with a clinically relevant request for healthcare by the patient (as is judged by the referring party).

Psychological interventions for tinnitus can potentially include a wide range of components but there is general consensus on the use of two of them. In particular, the provision of information and education about tinnitus and treatment options is consistently recommended across the guidelines although there is some variation in the specificity of the content that each provides. Second, specialised CBT for tinnitus is specifically recommended by all the guidelines except for Sweden which mentions it only in relation to the presence of stress, anxiety, or depression. 


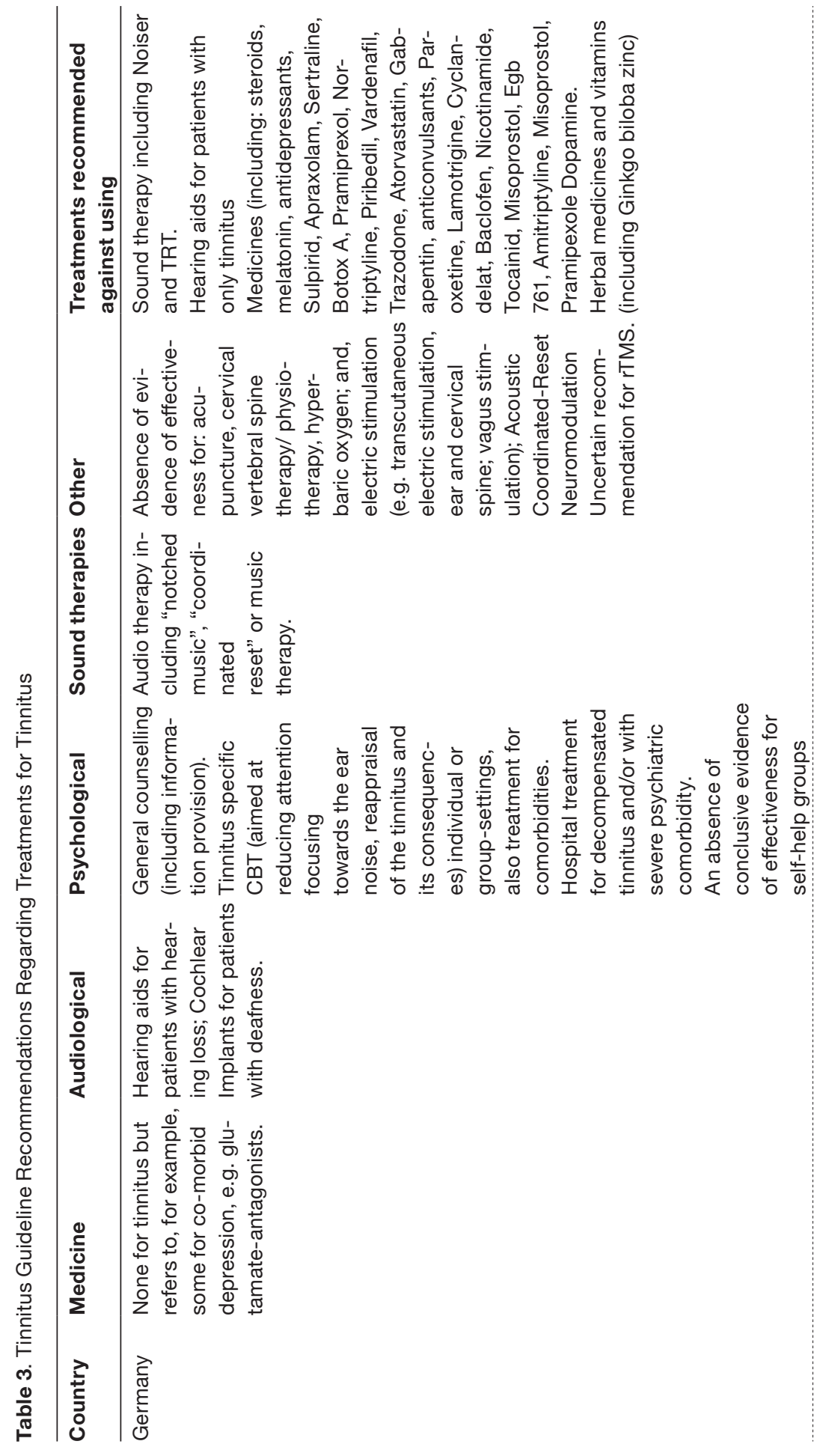




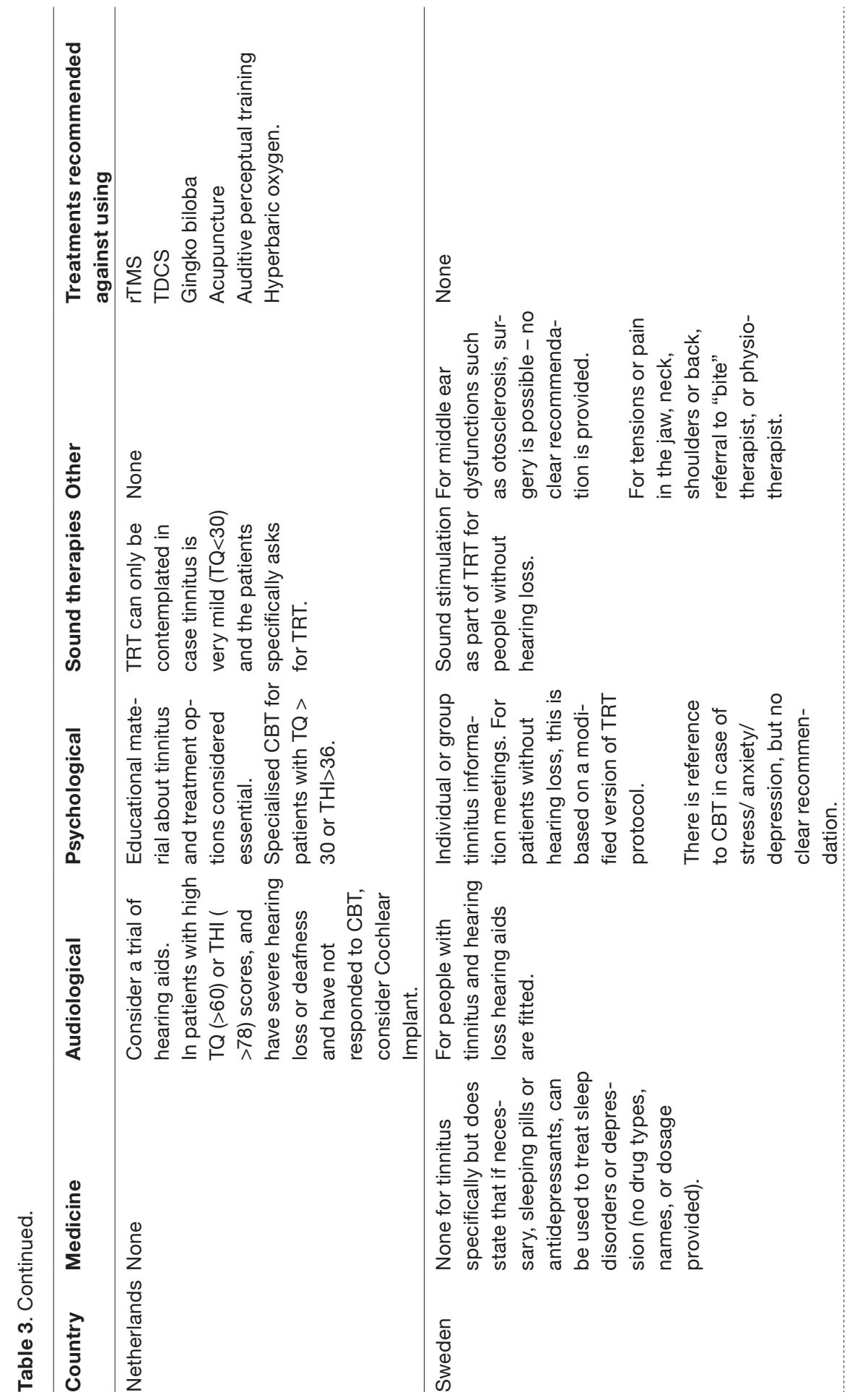




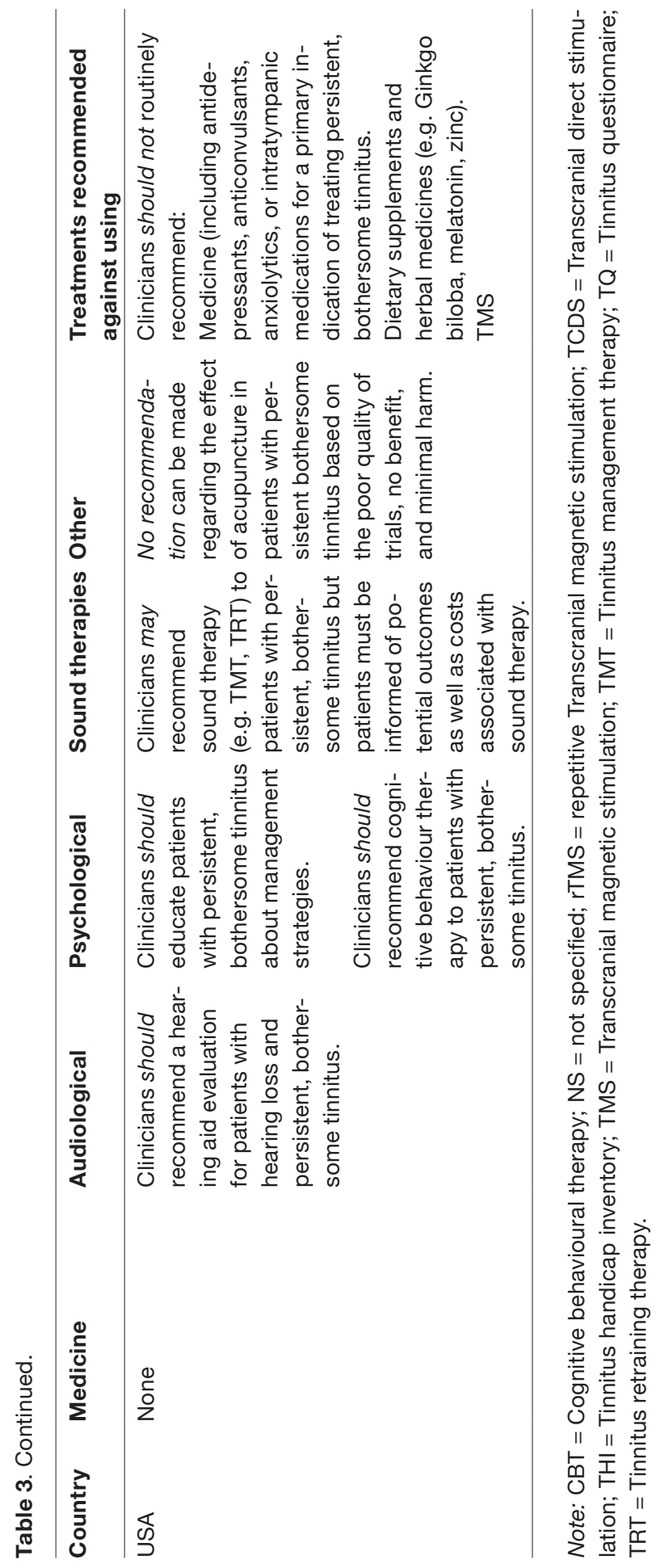


Least consistency exists across the guidelines in relation to TRT. Specifically, the Dutch guideline recommends that TRT can only be contemplated if tinnitus is very mild $(T Q<30)$ and the patients specifically asks for TRT, the American guideline indicates that sound therapies "may" be recommended to patients with tinnitus, while the Swedish guideline recommends that sound stimulation be used as part of TRT for people without hearing loss. The German guideline recommends the use of notched music therapy, but recommends against the use of TRT.

In relation to other less commonly used treatments (such as acupuncture or hyperbaric oxygen), the guidelines mostly indicate that there is an insufficient body of evidence to be able to make recommendations for or against their use.

Lastly, three guidelines (Germany, The Netherlands, and U.S.) either caution that there is insufficient evidence, or make additional recommendations against the use of transcranial magnetic stimulation (rTMS), transcranial direct current stimulation (tDCS), dietary supplements, neuromodulation treatments, and hearing aids for tinnitus patients without hearing loss.

\section{Summary of therapeutic recommendations regarding the treatment of subjective tinnitus}

- Provide information about tinnitus and treatment options (all guidelines)

- Use hearing aids only when patients also experience hearing loss (all guidelines)

- Specialised CBT for tinnitus should be offered to patients (three of four guidelines; Sweden refers to use of CBT in context of co-morbid anxiety or depression)

- There is a lack of consensus on the use of TRT for tinnitus

- Prescribed medicines and herbal supplements should not be used for the treatment of tinnitus (all guidelines)

- Treatment with TMS is recommended against by Dutch and U.S. guidelines, and German guidelines give an "uncertain" recommendation.

\section{Quality assessment of the guidelines}

The AGREE II tool was used by the authors who undertook data extraction of the respective guidelines and the summarised results are shown in Table 4. In general the domains of "stakeholder involvement" and "clarity of presentation" respectively by guideline developers were rated high (good quality). Conversely, ratings on the domain of "applicability" which refers to how the guidelines might be disseminated, implemented and evaluated were low. For the domains addressing the scope and purpose of the guidelines, rigour of development and editorial independence, a pattern emerged whereby the American, Dutch and German guidelines were rated considerably higher (AGREE II scores $>60 \%$ on all domains) than the Danish and Swedish guidelines (AGREE II scores $<60 \%$ on all domains). 
Table 4. Summary of AGREE II Domain Scores (\%) by Country

\begin{tabular}{lllllll}
\hline & $\begin{array}{l}\text { Scope \& } \\
\text { purpose }\end{array}$ & $\begin{array}{l}\text { Stakeholder } \\
\text { involvement }\end{array}$ & $\begin{array}{l}\text { Rigour of } \\
\text { development }\end{array}$ & $\begin{array}{l}\text { Clarity of } \\
\text { presentation }\end{array}$ & $\begin{array}{c}\text { Applicability } \\
\text { Editorial } \\
\text { independence }\end{array}$ \\
\hline Germany & 61 & 94 & 83 & 89 & 71 & 67 \\
Denmark & 52 & 44 & 24 & 59 & 2 & 17 \\
Netherlands & 81 & 100 & 97 & 100 & 9 & 100 \\
USA & 86 & 97 & 93 & 100 & 71 & 88 \\
Sweden & 42 & 42 & 1 & 33 & 2 & 13 \\
\hline Median & 61 & 94 & 83 & 89 & 9 & 67 \\
Average & 64 & 75 & 60 & 76 & 31 & 57 \\
\hline
\end{tabular}

\section{Discussion}

This systematic review aims to compare existing clinical guidelines for the assessment and treatment of subjective tinnitus in adults. Five guidelines, developed in the last 10 years within Europe, Scandinavia, and North America were included in the review. Although there are differences in some specific recommendations for assessment and treatment procedures across the guidelines, in general, commonalities across guidelines were high. The fact that there are differences in some of the recommendations is not surprising and appears to reflect the relatively young state of the field and the evolving nature of assessment and treatments for subjective tinnitus - a symptom with a high level of heterogeneity. On the other hand, the level of agreement, for example, in the recommendation of specialised cognitive behavioural therapy reflects the growing evidence base for the effectiveness of this treatment to alleviate patients' distress and impairment, even though significant changes in the tinnitus percept itself as a result of CBT have been proposed, though not yet assessed across studies.

When the methods of the development of guidelines were reported, it was clear that the respective groups were making efforts to be transparent, systematic, and using the best available evidence base, and frequently linking recommendations to specific research literature. For example, systematic reviews and meta-analyses were referred to whenever available to inform recommendations. It should be noted though that there is a lack of high quality studies or powered randomised trials of some treatments either for practical or methodological reasons. Regardless, the strengths and limitations of the evidence for particular recommendations were included for the majority of the guidelines and thus enable the user/reader to make informed decisions about following the recommended actions. Furthermore, target users were generally clearly defined and the development groups were comprised of a range of the health professionals often involved in the assessment and treatment of tinnitus. These two factors are important not only for providing expert input into the guideline, but also for garnering "buy-in" from potential users of the guidelines and focussing the content. 


\section{Differences between the guidelines}

Differences in recommended assessment procedures tend to relate to specific techniques (questionnaires, diagnostic tests, types of scanning techniques) rather than general principles (e.g. trying to establish tinnitus severity, hearing loss, psychosocial problem(s), or the presence or absence of severe physical pathology that might be causing the tinnitus. Differences related to, for example, the recommended questionnaires for assessing tinnitus related interference and distress. While all the guidelines referred to the THI (the German guideline indirectly refers to this), only the American, Dutch and German guidelines referred to the TQ. Recommendations for specific questionnaires to measure psychological distress (especially symptoms of anxiety and depression) also varied with some guidelines not mentioning any (e.g. United States) and others such as the Dutch and Swedish guidelines which referred to the HADS. Differences also existed between the recommendations to assess loudness discomfort levels with the American and Dutch guidelines not recommending the use of such tests while the other guidelines did.

With regard to treatments, differences are found primarily regarding recommendations for the use of sound therapies. TRT specifically is not recommended by the German guideline, conditionally by the Dutch guideline and the American guideline indicates that clinicians "may" recommend it; TRT is currently being tested in a large multicentre trial in the U.S. (clinical trials ID: NCT01177137). A lack of evidences about other treatments such as acupuncture, hyperbaric oxygen and some herbal supplements leads most groups to recommend against them. The American guideline though is more cautious and simply states that because there is a lack of evidence they can neither recommend for or against the use of such treatments.

Differences in the recommendations of assessment and treatment procedures could be explained by a combination of factors including the time of the development of the guideline and availability of translated versions of the questionnaires (e.g. the TFI was published in 2012 which was after that of the Danish and Swedish guidelines), the known psychometric properties of the questionnaires themselves [e.g. concerns have been raised about the cross-cultural use of the HADS (Maters, Sanderman, Kim, \& Coyne, 2013)], and the different methods used to reach consensus by the different guideline groups.

\section{Consistencies across the guidelines}

Across the guidelines consensus appears to exist on a number of important general features of assessment relating to subjective tinnitus. Specifically, there is consensus about the initial need for excluding a physical cause of the tinnitus, conducting an audiometric assessment of the patient, using standardised questionnaires to measure degrees of tinnitus related distress, and when relevant, making referrals for further psychological assessment.

Regarding the therapeutic recommendations for the treatment of subjective tinnitus, all guidelines recommend against the use of medicines for the treatment of the tinnitus specifically but note that medicines are appropriate for treating co-morbid conditions. There is also agreement in the recommendations to use hearing aids for patients 
experiencing hearing loss and CBT to facilitate adjustment to the symptom, alleviate distress and tinnitus-related interference in daily life.

As a group of tinnitus researchers and clinicians, we endorse the specific principles and practices of assessment and treatment that are consistently found across the guidelines. Further, while a treatment for removing the tinnitus percept does not exist, we reiterate the importance of providing patients with bothersome tinnitus, evidence based cost-effective treatment(s) in a way (such as stepped care) that is minimally burdensome to the patient. That is patients who are assessed as having relatively little tinnitus related distress and interference should receive less intensive treatment in the first instance, than someone who is assessed as having severe levels of distress and interference in activities of daily living.

\section{Strengths and limitations of the review}

There are two critical factors that affect the conclusions that can be drawn from the included guidelines. Firstly, and as with all systematic reviews, the search strategy and inclusion criteria used determine what is located and subsequently included. In this review, we used the search terms "tinnitus" and "guideline" to conduct the search in a wide range of databases, repositories of clinical guidelines, and search engines, with the intention of being focussed enough to identify the most relevant documents within a manageable number of search results. Only including the term "guideline" though might have resulted in relevant documents, albeit not called "guidelines", being omitted from search results. Similarly, our use of inclusion/exclusion criteria that led to the decision to exclude documents such as the TRI flowchart could be problematic as it (the TRI flowchart) is a comprehensive document potentially used in many situations to inform assessment and treatment decisions.

To minimise the risk of omitting relevant search results we contacted a range of international experts and members of guideline development groups. In addition to this, we conducted hand searches of the references lists of included guidelines for relevant sources. We also recruited native speakers to extract data from the respective guidelines in an effort to ensure that data collection was as accurate as possible. It is possible, that different search and inclusion criteria might have led to different documents being included. However, given the large range of assessment and treatment options and the limited evidence base around many treatments in particular, it is unlikely that our conclusions would differ significantly if further guidelines had been identified at this time. Future systematic reviews though will be able to use this as a reference point.

\section{Implications and conclusions}

As researchers from around the world are collecting and making efforts to better understand the heterogeneity of subjective tinnitus in adults and systematically evaluate assessment and treatment options, we have, for the first time, described the major similarities and differences between existing clinical guidelines for subjective tinnitus in adults. The results reveal true guidelines from only five countries and thus highlight a need to develop guidelines that are endorsed by the range of professionals involved in assessing and treating tinnitus. Although we do not place a great deal of weight on 
the quality assessment ratings of the guidelines, they do suggest that there is room for improvement particularly with regard to implementation and evaluation. The absence of guidelines contributes to the variations that exist in assessment and treatment of tinnitus internationally.

While it would be tempting to do so, it is beyond the scope of this paper to formulate a new or composite guideline based on the results that we have obtained. Instead, the results of this review in conjunction with those from a survey of European tinnitus healthcare providers and researchers (Cima et al., 2016) will form the basis of further work on the development of a set of European clinical guidelines for the assessment and treatment of tinnitus being undertaken by the COST-action TINNET: Working Group I 'Clinical'. As with existing clinical guidelines, attention will need to be given to how the future European guideline is disseminated, subsequently evaluated, and the implications for resource management considered. We expect it to be challenging task but one that will hopefully result in a more reliable and equitable assessment and treatment of tinnitus patients across Europe. 


\section{References}

Andersson, G., Juris, L., Classon, E., Fredrikson, M., \& Furmark, T. (2006). Consequences of suppressing thoughts about tinnitus and the effects of cognitive distraction on brain activity in tinnitus patients. Audiol Neurootol, 11(5), 301-309. doi:10.1159/000094460

Audiology Australia. (2013). Audiology Australia Professional Practice Standards - Part B Clinical Standards. Retrieved from Melbourne, Australia:

Baguley, D., Andersson, G., McFerran, D., \& McKenna, L. (Eds.). (2013). Tinnitus: A Multidisciplinary Approach (2nd ed.). West Sussex, UK: John Wiley \& Sons, Ltd.

Baguley, D., McFerran, D., \& Hall, D. (2013). Tinnitus. Lancet, 382(9904), 1600-1607. doi:10.1016/s0140-6736(13)60142-7

Biesinger, E., Del Bo, L., De Ridder, D., Goodey, R., Herraiz, C., Kleinjung, T., . . . Searchfield, G. (2010). Algorithm for the Diagnostic \& Therapeutic Management of Tinnitus. Retrieved from http://www.tinnitusresearch.org/en/documents/downloads/ TRI_Tinnitus_Flowchart.pdf

Brouwers, M. C., Kho, M. E., Browman, G. P., Burgers, J. S., Cluzeau, F., Feder, G., . . . Zitzelsberger, L. (2010). AGREE II: advancing guideline development, reporting and evaluation in health care. CMAJ, 182(18), E839-842. doi:10.1503/cmaj.090449

Cima, R. F. F., Crombez, G., \& Vlaeyen, J. W. (2011). Catastrophizing and fear of tinnitus predict quality of life in patients with chronic tinnitus. Ear Hear, 32(5), 634-641. doi:10.1097/AUD.0b013e31821106dd

Cima, R. F. F., Haider, H., Mazurek, B., Cederroth, C. R., Lapira, A., Kikidis, D., \& Norena, A. (2016). TINNET COST Action BM1306 - Clinical WG1: Establishment of a standard for Tinnitus; patient assessment, characterization, and treatment options. Paper presented at the 10th International Tinnitus Research Initiative Conference, Nottingham, England. https://tri2016.ihr.mrc.ac.uk/downloads/programme-abstracts-a4-15.pdf

Cima, R. F. F., Maes, I. H., Joore, M. A., Scheyen, D. J., El Refaie, A., Baguley, D. M., . . . Vlaeyen, J. W. (2012). Specialised treatment based on cognitive behaviour therapy versus usual care for tinnitus: a randomised controlled trial. Lancet, 379(9830), 19511959. doi:10.1016/s0140-6736(12)60469-3

Davis, A., \& El Refaie, A. (2000). Epidemiology of Tinnitus. In R. S. Tyler (Ed.), Handbook of Tinnitus (pp. 1-24). San Diego: Singular thompson Learning.

Department of Health. (2009). Provision of Services for Adults with Tinnitus: A Good Practice Guide Retrieved from London, England:

Dutch Association for Ear Nose Throat and Head surgery [Nederlandse Vereniging voor Keel - Neus - Oor heel kunde en Heelkunde van het Hoofd -Halsgebied]. (2016). Guideline Tinnitus [Richtlijn Tinnitus]. Retrieved from Utrecht, Netherlands: https:// richtlijnendatabase.nl/richtlijn/tinnitus/tinnitus_-_startpagina.html 
Erlandsson, S. I., \& Hallberg, L. R. (2000). Prediction of quality of life in patients with tinnitus. Br J Audiol, 34(1), 11-20.

Feldmann, H. (1984). Tinnitus masking curves (updates and review). The Journal of Laryngology \& Otology, 98(S9), 157-160. doi:10.1017/S1755146300090375

Field, M. J., \& Lohr, K. N. (Eds.). (1990). Clinical Practice Guidelines: Directions for a New Program. Washington DC: National Academy of Sciences.

Fujii, K., Nagata, C., Nakamura, K., Kawachi, T., Takatsuka, N., Oba, S., \& Shimizu, H. (2011). Prevalence of tinnitus in community-dwelling Japanese adults. J Epidemiol, 21(4), 299-304.

Goebel, G., \& Hiller, W. (1994). The tinnitus questionnaire. A standard instrument for grading the degree of tinnitus. Results of a multicenter study with the tinnitus questionnaire. HNO, 42(3), 166-172.

Goebel, G., \& Hiller, W. (2001). Verhaltensmedizinische Tinnitus-Diagnostik. Eine praktische Anleitung zur Erfassung medizinischer und psychologischer Merkmale mittels des Strukturierten Tinnitus-Interviews (STI) . Göttingen: Hogrefe.

Hallam, R. S., Jakes, S. C., \& Hinchcliffe, R. (1988). Cognitive variables in tinnitus annoyance. Br J Clin Psychol, 27, 213-222. doi:10.1111/j.2044-8260.1988.tb00778.x

Handscomb, L. E., Hall, D. A., Shorter, G. W., \& Hoare, D. J. (2017). Positive and Negative Thinking in Tinnitus: Factor Structure of the Tinnitus Cognitions Questionnaire. Ear Hear, 38(1), 126-132. doi:10.1097/AUD.0000000000000365

Hoare, D. J., Gander, P. E., Collins, L., Smith, S., \& Hall, D. A. (2012). Management of tinnitus in English NHS audiology departments: an evaluation of current practice. Journal of Evaluation in Clinical Practice, 18(2), 326-334. doi:10.1111/j.13652753.2010.01566.x

Hoare, D. J., \& Hall, D. (2011). Clinical guidelines and practice: a commentary on the complexity of tinnitus management. Eval Health Prof, 34(4), 413-420. doi:10.1177/0163278710390355

Hoekstra, C. E., Wesdorp, F. M., \& van Zanten, G. A. (2014). Socio-demographic, health, and tinnitus related variables affecting tinnitus severity. Ear Hear, 35(5), 544-554. doi:10.1097/aud.0000000000000045

Idrizbegovic, E., Kjerulf, E., \& Team for Diagnostics Hearing Habilitation Children and Youth and Hearing Rehabilitation for Adults. (2011). Tinnitus Care program [Tinnitus Vårdprogram]. Karolinska Institute. Stockholm, Sweden. Retrieved from http://www. viss.nu/Global/Bilagor/onh_horsel/vardprogram_tinnitus_20111216.pdf

Jørgensen, H. S., Amt, F., Nemholt, S. S., Kristensen, R., \& Ellesøe, H. (2007). Guidance for diagnosing tinnitus and hyperacusis [Vejledning for udredning af tinnitus og hyperakusis] (9788789925110). Retrieved from Denmark: 
Kamalski, D. M., Hoekstra, C. E., van Zanten, B. G., Grolman, W., \& Rovers, M. M. (2010). Measuring disease-specific health-related quality of life to evaluate treatment outcomes in tinnitus patients: a systematic review. Otolaryngol Head Neck Surg, 143(2), 181-185. doi:10.1016/j.otohns.2010.03.026

Kim, H. J., Lee, H. J., An, S. Y., Sim, S., Park, B., Kim, S. W., . . Choi, H. G. (2015). Analysis of the prevalence and associated risk factors of tinnitus in adults. PLoS ONE, 10(5), e0127578. doi:10.1371/journal.pone.0127578

Kleinstäuber, M., Jasper, K., Schweda, I., Hiller, W., Andersson, G., \& Weise, C. (2013). The role of fear-avoidance cognitions and behaviors in patients with chronic tinnitus. Cogn Behav Ther, 42(2), 84-99. doi:10.1080/16506073.2012.717301

Kuk, F. K., Tyler, R. S., Russell, D., \& Jordan, H. (1990). The psychometric properties of a tinnitus handicap questionnaire. Ear Hear, 11(6), 434-445.

Langguth, B., \& Elgoyhen, A. B. (2012). Current pharmacological treatments for tinnitus. Expert Opin Pharmacother, 13(17), 2495-2509. doi:10.1517/14656566.2012.739608

Langguth, B., Kleinjung, T., \& Landgrebe, M. (2011). Severe tinnitus and depressive symptoms: a complex interaction. Otolaryngology--head and neck surgery : official journal of American Academy of Otolaryngology-Head and Neck Surgery, 145(3), 519; author reply 520. doi:10.1177/0194599811411851

Langguth, B., Kreuzer, P. M., Kleinjung, T., \& De Ridder, D. (2013). Tinnitus: causes and clinical management. Lancet Neurol, 12(9), 920-930. doi:10.1016/s1474-4422(13)70160-1

Martinez-Devesa, P., Perera, R., Theodoulou, M., \& Waddell, A. (2010). Cognitive behavioural therapy for tinnitus. Cochrane Database Syst Rev(9), CD005233. doi:10.1002/14651858.CD005233.pub3

Maters, G. A., Sanderman, R., Kim, A. Y., \& Coyne, J. C. (2013). Problems in crosscultural use of the hospital anxiety and depression scale: "no butterflies in the desert”. PLoS ONE, 8(8), e70975. doi:10.1371/journal.pone.0070975

McKenna, L., Handscomb, L., Hoare, D. J., \& Hall, D. A. (2014). A scientific cognitivebehavioral model of tinnitus: novel conceptualizations of tinnitus distress. Front Neurol, 5, 196. doi:10.3389/fneur.2014.00196

Meikle, M. B., Henry, J. A., Griest, S. E., Stewart, B. J., Abrams, H. B., McArdle, R., . . . Vernon, J. A. (2012). The tinnitus functional index: development of a new clinical measure for chronic, intrusive tinnitus. Ear Hear, 33(2), 153-176. doi:10.1097/ AUD.0b013e31822f67c0

Newman, C. W., Jacobson, G. P., \& Spitzer, J. B. (1996). Development of the tinnitus handicap inventory. Archives of Otolaryngology-Head \& Neck Surgery, 122(2), 143148. doi:10.1001/archotol.1996.01890140029007

Newman, C. W., Sandridge, S. A., \& Jacobson, G. P. (1998). Psychometric adequacy of the Tinnitus Handicap Inventory (THI) for evaluating treatment outcome. J Am Acad Audiol, 9(2), 153-160. 
Ruszczynski, M., Horvath, A., Dziechciarz, P., \& Szajewska, H. (2016). Cow's milk allergy guidelines: a quality appraisal with the AGREE II instrument. Clin Exp Allergy, 46(9), 1236-1241. doi:10.1111/cea.12784

Sanclemente, G., Acosta, J. L., Tamayo, M. E., Bonfill, X., \& Alonso-Coello, P. (2014). Clinical practice guidelines for treatment of acne vulgaris: a critical appraisal using the AGREE II instrument. Arch Dermatol Res, 306(3), 269-277. doi:10.1007/s00403-013-1394-x

The Association of the Scientific Medical Societies. (2015). German S3 guideline 017/064: Chronic tinnitus [AWMF-Register Nr. 017/064 Klasse: S3 Chronischer Tinnitus]. Retrieved from Germany:

Tunkel, D. E., Bauer, C. A., Sun, G. H., Rosenfeld, R. M., Chandrasekhar, S. S., Cunningham, E. R., . . Whamond, E. J. (2014). Clinical Practice Guideline: Tinnitus. Otolaryngol Head Neck Surg, 151(2 suppl), S1-S40. doi:10.1177/0194599814545325

Wilson, P. H., Henry, J., Bowen, M., \& Haralambous, G. (1991). Tinnitus Reaction Questionnaire Psychometric Properties of a Measure of Distress Associated With Tinnitus. Journal of Speech, Language, and Hearing Research, 34(1), 197-201. doi:10.1044/jshr.3401.197

Zigmond, A. S., \& Snaith, R. P. (1983). The hospital anxiety and depression scale. Acta Psychiatr Scand, 67(6), 361-370. 
Systematic review of clinical guidelines for tinnitus 


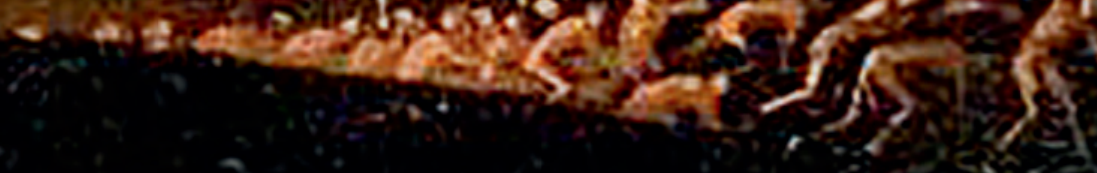

ats

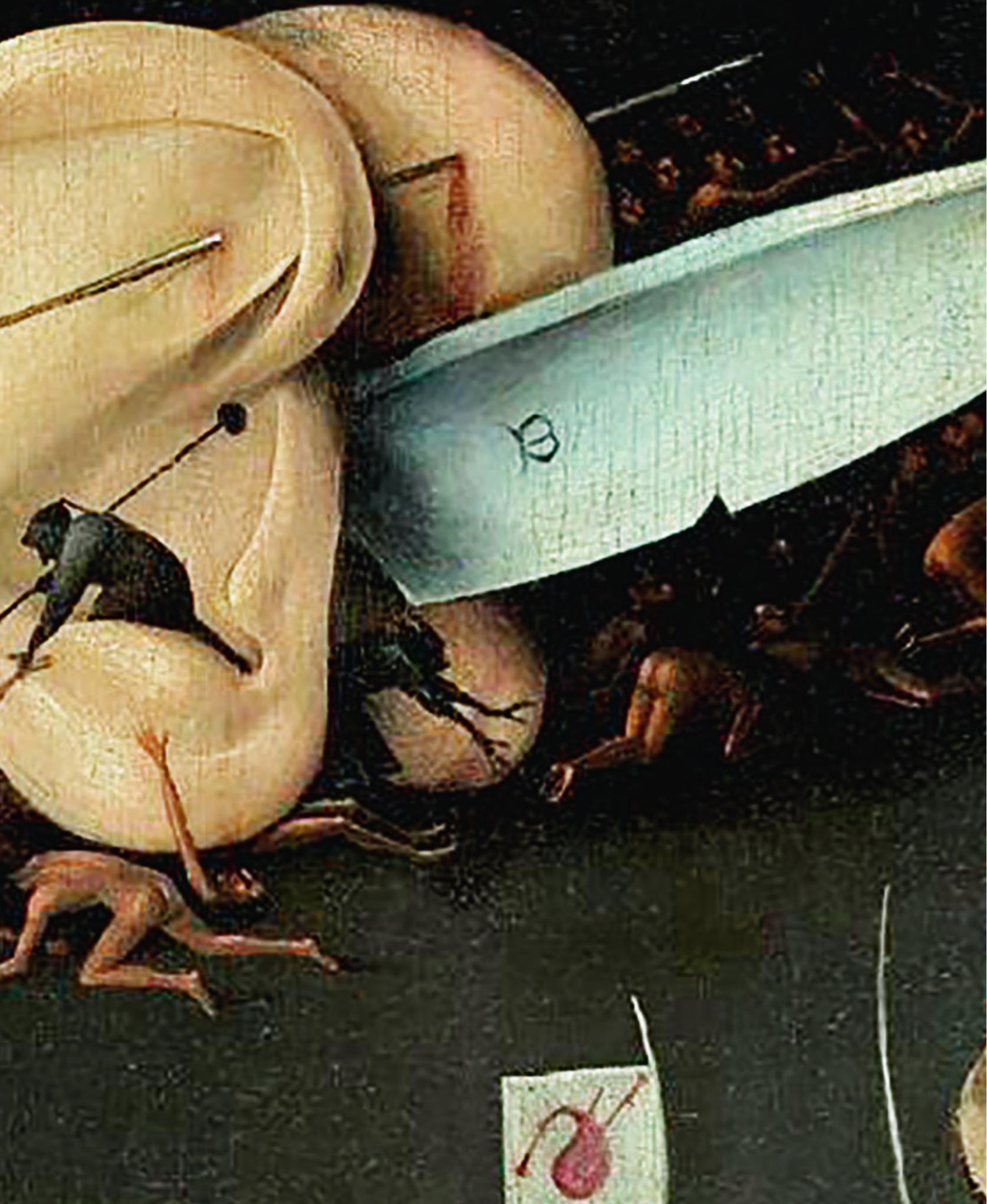




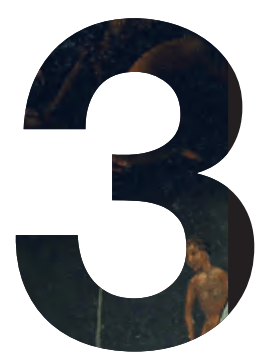

\section{SYSTEMATIC REVIEW AND \\ META-ANALYSIS OF CBT FOR}

\section{TINNITUS IN ADULTS}

Published as: Citation example: Fuller T, Cima R, Langguth B, Mazurek B, Vlaeyen JWS, Hoare DJ. Cognitive behavioural therapy for tinnitus. Cochrane Database of Systematic Reviews 2017, Issue 4. Art. No.: CD012614.

DOI: 10.1002/14651858.CD012614.

Note: Citations and references have been kept in the Cochrane style as used in the published version of this chapter. 


\section{Abstract}

\section{Background}

Tinnitus affects up to $21 \%$ of the adult population with an estimated $1 \%$ to $3 \%$ experiencing severe problems. Cognitive behavioural therapy (CBT) is a collection of psychological treatments based on the cognitive and behavioural traditions in psychology and often used to treat people suffering from tinnitus.

\section{Objectives}

To assess the effects and safety of CBT for tinnitus in adults.

\section{Search methods}

The Cochrane ENT Information Specialist searched the ENT Trials Register; CENTRAL (2019, Issue 11); Ovid MEDLINE; Ovid Embase; CINAHL; Web of Science; ClinicalTrials. gov; ICTRP and additional sources for published and unpublished trials. The date of the search was 25 November 2019.

\section{Selection criteria}

Randomised controlled trials (RCTs) of CBT versus no intervention, audiological care, tinnitus retraining therapy or any other active treatment in adult participants with tinnitus.

\section{Data collection and analysis}

We used the standard methodological procedures expected by Cochrane. Our primary outcomes were the impact of tinnitus on disease-specific quality of life and serious adverse effects. Our secondary outcomes were: depression, anxiety, general healthrelated quality of life, negatively biased interpretations of tinnitus and other adverse effects. We used GRADE to assess the certainty of evidence for each outcome.

\section{Main results}

We included 28 studies (mostly from Europe) with a total of 2733 participants. All participants had had tinnitus for at least three months and their average age ranged from 43 to 70 years. The duration of the CBT ranged from 3 to 22 weeks and it was mostly conducted in hospitals or online.

There were four comparisons and we were interested in outcomes at end of treatment, and 6 and 12 months follow-up. The results below only refer to outcomes at end of treatment due to an absence of evidence at the other follow-up time points.

\section{CBT versus no intervention/wait list control}

Fourteen studies compared CBT with no intervention/wait list control. For the primary outcome, CBT may reduce the impact of tinnitus on quality of life at treatment end (standardised mean difference (SMD) -0.56, 95\% confidence interval (CI) -0.83 to -0.30 ; 10 studies; 537 participants; low certainty). Re-expressed as a score on the Tinnitus Handicap Inventory (THI; range 0 to 100) this is equivalent to a score 10.91 points lower in the CBT group, with an estimated minimal clinically important difference (MCID) for 
this scale being 7 points. Seven studies, rated as moderate certainty, either reported or informed us via personal communication about serious adverse effects. CBT probably results in little or no difference in adverse effects: six studies reported none and in one study one participant in the CBT condition worsened (risk ratio (RR) 3.00, 95\% Cl 0.13 to 69.87). For the secondary outcomes, CBT may result in a slight reduction in depression (SMD -0.34, 95\% Cl-0.60 to -0.08; 8 studies; 502 participants; low certainty). However, we are uncertain whether CBT reduces anxiety, improves health-related quality of life or reduces negatively biased interpretations of tinnitus (all very low certainty). From seven studies, no other adverse effects were reported (moderate certainty).

\section{CBT versus audiological care}

Three studies compared CBT with audiological care. CBT probably reduces the impact of tinnitus on quality of life when compared with audiological care as measured by the THI (range 0 to 100; mean difference (MD) $-5.65,95 \% \mathrm{Cl}-9.79$ to -1.50 ; 3 studies; 444 participants) (moderate certainty; $M C I D=7$ points). No serious adverse effects occurred in the two included studies reporting these, thus risk ratios were not calculated (moderate certainty). The evidence suggests that CBT may slightly reduce depression but may result in little or no difference in anxiety or health-related quality of life (all low certainty) when compared with audiological care. CBT may reduce negatively biased interpretations of tinnitus when compared with audiological care (low certainty). No other adverse effects were reported for either group (moderate certainty).

\section{CBT versus tinnitus retraining therapy (TRT)}

One study compared CBT with TRT (including bilateral sound generators as per TRT protocol). CBT may reduce the impact of tinnitus on quality of life as measured by the THI when compared with TRT (range 0 to 100 ) (MD -15.79, 95\% Cl -27.91 to $-3.67 ; 1$ study; 42 participants; low certainty). For serious adverse effects three participants deteriorated during the study: one in the CBT $(n=22)$ and two in the TRT group $(n=20)$ (RR 0.45, 95\% Cl 0.04 to 4.64; low certainty). We are uncertain whether CBT reduces depression and anxiety or improves health-related quality of life (low certainty). CBT may reduce negatively biased interpretations of tinnitus. No data were available for other adverse effects.

\section{CBT versus other active control}

Sixteen studies compared CBT with another active control (e.g. relaxation, information, Internet-based discussion forums). CBT may reduce the impact of tinnitus on quality of life when compared with other active treatments (SMD $-0.30,95 \% \mathrm{Cl}-0.55$ to $-0.05 ; 12$ studies; 966 participants; low certainty). Re-expressed as a THI score this is equivalent to 5.84 points lower in the CBT group than the other active control group (MCID $=7$ points). One study reported that three participants deteriorated: one in the CBT and two in the information only group (RR $1.70,95 \% \mathrm{Cl} 0.16$ to 18.36 ; low certainty). CBT may reduce depression and anxiety (both low certainty). We are uncertain whether CBT improves health-related quality of life compared with other control. CBT probably reduces negatively biased interpretations of tinnitus compared with other treatments. No data were available for other adverse effects. 


\section{Authors' conclusions}

CBT may be effective in reducing the negative impact that tinnitus can have on quality of life. There is, however, an absence of evidence at 6 or 12 months follow-up. There is also some evidence that adverse effects may be rare in adults with tinnitus receiving $C B T$, but this could be further investigated. CBT for tinnitus may have small additional benefit in reducing symptoms of depression although uncertainty remains due to concerns about the quality of the evidence. Overall, there is limited evidence for CBT for tinnitus improving anxiety, health-related quality of life or negatively biased interpretations of tinnitus.

\section{Plain language summary}

\section{Cognitive behavioural therapy for adults with tinnitus}

\section{What is the aim of this review?}

The aim of this Cochrane Review was to find out if cognitive behavioural therapy (CBT) is effective for tinnitus. Cochrane researchers collected and analysed all relevant studies to answer this question.

\section{Key messages}

There is some low- to moderate-certainty evidence that CBT may reduce the negative impact that tinnitus can have on quality of life at the end of treatment, with few or no adverse effects (although further research on this is needed).

\section{What was studied in the review?}

Tinnitus is the perception of sound in the ear or head without any outside source. It is often described as a ringing, hissing, buzzing or whooshing sound. Tinnitus is mostly managed with education and/or counselling, relaxation therapy, tinnitus retraining therapy and ear-level sound generators or hearing aids. CBT is a form of talking therapy that aims to change the patient's emotional and/or behavioural response to their tinnitus. This review looked at studies of CBT for adults who had had tinnitus for at least three months. Participants in the control groups either received no intervention, audiological (hearing) care, tinnitus retraining therapy or another type of treatment. The review authors studied the effect of CBT on tinnitus-related quality of life, adverse effects, depression, anxiety, general quality of life and negatively biased interpretations of tinnitus.

\section{What are the main results of the review?}

We found 28 relevant studies, mostly from Europe, with a total of 2733 participants. The participants receiving CBT had treatment for between three and 22 weeks (mostly in clinics or online).

When CBT was compared to no intervention there was low-certainty evidence that CBT may reduce the negative impact of tinnitus on quality of life at the end of treatment. It is not known whether this effect persists in the longer term (six or 12 months). There were few or no adverse effects (only one adverse effect was reported in one participant among 
seven studies). CBT may also slightly reduce depression (low-certainty evidence) and may reduce anxiety, although this finding is very uncertain. It is also uncertain whether CBT improves general quality of life or negatively biased interpretations of tinnitus.

Compared to audiological care, tinnitus retraining therapy and other types of treatment, there were findings that CBT probably reduces the negative impact of tinnitus on quality of life. The certainty of this evidence ranged from moderate to low. Where reported, there were few adverse effects and no significant differences between the groups. For depression, anxiety and general quality of life the results were more mixed and the evidence less certain. There is moderate-certainty evidence that CBT may reduce negatively biased interpretations of tinnitus compared to other types of treatment, but compared to audiological care and tinnitus retraining therapy the evidence is less certain.

\section{How up to date is this review?}

The review authors searched for studies that had been published up to November 2019. 


\section{Background}

The following paragraphs and Description of the condition are based on the Cochrane Review 'Amplification with hearing aids for patients with tinnitus and co-existing hearing loss' and reproduced with permission (Hoare 2014).

Tinnitus is defined as the perception of sound in the absence of a corresponding auditory source (Jastreboff 2004). It is typically described by those who experience it as a ringing, hissing, buzzing or whooshing sound and is thought to result from abnormal neural activity and connectivity in auditory and non-auditory pathways, which is interpreted by the brain as sound (Elgoyhen 2015; Shore 2016). Tinnitus can be either objective or subjective.

Objective tinnitus is estimated to occur in up to $10 \%$ of people with tinnitus seeking help (Kircher 2008), and refers to the perception of sound that can also be heard by the examiner (Roberts 2010). Objective forms include heartbeat synchronous pulsatile tinnitus and they usually have a detectable cause such as arteriovenous malformation, carotid stenosis or dissections (Langguth 2013). Specific medication or surgical treatment can lead to the cessation of the objective tinnitus percept (Kleinjung 2016).

Most commonly, however, tinnitus is subjective, meaning that the sound is only heard by the person experiencing it and no source of the sound can be identified (Jastreboff 1988). Subjective tinnitus (the focus of this review) is estimated to affect up to $21 \%$ of the general adult population, increasing to as many as $30 \%$ of adults over 50 years of age (Davis 2000; Gallus 2015; Kim 2015). It can be experienced acutely, recovering spontaneously within minutes to weeks. However, it can become chronic and is unlikely to resolve spontaneously when experienced for three months or more (Hahn 2008; Hall 2011; Rief 2005). In 1\% to $3 \%$ of the population tinnitus causes severe problems with daily life functioning (Davis 2000; Kim 2015). Although a range of psychological, sound, electrical and electromagnetic therapies have been developed, currently there is no reliable cure for subjective tinnitus.

In England alone there are an estimated 3/4 million General Practitioner consultations every year where the primary complaint is tinnitus (El-Shunnar 2011), equating to a major burden on healthcare services. For many people tinnitus is persistent and troublesome, and has disabling effects such as insomnia, difficulty concentrating, difficulties in communication and social interaction, and negative emotional responses such as anxiety and depression (Andersson 2009; Cima 2011b; Crönlein 2007; Langguth 2011; Marciano 2003; Zirke 2013a; Zirke 2013b). In approximately $90 \%$ of cases, chronic tinnitus is co-morbid with some degree of hearing loss, which may confound these disabling effects (Fowler 1944; Sanchez 2002). An important implication of this in clinical research is that outcome measures need to distinguish benefits specific to the tinnitus signal itself and related aspects such as impairments in communication, emotional processing and social interaction, which all play a relevant role in quality of life. 
For the purposes of this review we will use 'the impact of tinnitus on quality of life' (or tinnitusrelated quality of life) as a collective term for the cognitive, emotional and behavioural consequences/sequelae that people living with chronic tinnitus experience. Additionally, unless otherwise noted, we will refer to subjective tinnitus simply as tinnitus.

\section{Description of the condition}

\section{Pathophysiology}

Most people with chronic tinnitus have some degree of hearing loss (Ratnayake 2009), and the prevalence of tinnitus increases with greater hearing loss (Han 2009; Martines 2010). Converging evidence from animal models and studies of human tinnitus sufferers indicates that, while cochlear damage is a trigger, most cases of tinnitus are generated by changes that take place in central auditory pathways when auditory neurons lose their input from the ear (Noreña 2011). Forms of neural plasticity underlie these neural changes, which include: increased spontaneous activity and neural gain in deafferented central auditory structures; increased synchronous activity in these structures; and changes in network behaviour in non-auditory brain regions. These changes have been detected by functional imaging of individuals with tinnitus and corroborated by animal investigations (Eggermont 2014; Elgoyhen 2015). (Additional detail is provided in Appendix 1).

A complication in understanding the pathophysiology of tinnitus is that not all people with hearing loss have tinnitus and not all people with tinnitus have a clinically significant hearing loss. Other variables, such as the profile of a person's hearing loss, may account for differences in their tinnitus report. For example, König 2006 found that the maximum slope within audiograms was higher in people with tinnitus than in people with hearing loss who do not have tinnitus, despite the 'non-tinnitus' group having the greater mean hearing loss. Also the additional involvement of non-auditory areas of the brain, particularly areas associated with awareness and salience detection, can explain why some people with hearing loss develop tinnitus whereas others do not (de Ridder 2011; de Ridder 2014).

Whether tinnitus is perceived as bothersome or not may be related to the additional involvement of emotion processing areas (Rauschecker 2010; Schecklmann 2013; Vanneste 2012). Accordingly, some models have proposed that tinnitus reflects "an emergent property of multiple parallel dynamically changing and partially overlapping sub-networks". This suggests that various brain networks associated with memory and emotional processing are involved in tinnitus and that the degree of involvement of the different networks reflects the variable aspects of an individual's tinnitus (de Ridder 2011; de Ridder 2014; Elgoyhen 2015).

\section{Psychological models of tinnitus}

In addition to the physiological data and models of tinnitus, psychological models have been developed to explain how and why some people experience a negative impact of tinnitus on quality of life. Psychological models of tinnitus include those developed by Hallam, which applies the concept of habituation (Hallam 1984); Jastreboff, whose model features classical conditioning mechanisms (Jastreboff 1988; Jastreboff 1990); and the 
cognitive behavioural models of McKenna 2014, Cima 2011b and Kleinstauber 2013 (Appendix 2). These psychological models underpin the rationale and development of cognitive behavioural interventions for reducing the impact of tinnitus on quality of life.

\section{Diagnosis and clinical management of tinnitus}

There is no universal internationally established standard procedure for the diagnosis or management of tinnitus. However, common across the (few) published practice guidelines is the use or recommendation of self-report questionnaires to assess tinnitus and its impact on patients by measuring severity, quality of life, depression or anxiety (Fuller 2017a). Psychoacoustic measures of tinnitus (pitch, loudness, minimum masking level) are also used in patient assessment but do not correlate well with self-reported measures of tinnitus annoyance (Hiller 2006). Instead they represent measurements of tinnitus that can be useful in patient counselling by, for example, demonstrating changes (or stability) in the individual's perception of the tinnitus over time (Department of Health 2009). No objective measures of tinnitus currently exist and so researchers and clinicians are reliant upon patient self-report measures (usually questionnaires with Likert-type or visual analogue scales) to record any changes in tinnitus related quality of life or other general health effects of therapy (Appendix 3 ). The previous Cochrane Review of cognitive behavioural therapy for tinnitus used self-reported, subjective tinnitus loudness as the primary outcome measure (Martinez-Devesa 2010). That review and others like it have consistently reported that there are generally weak (if any) effects of the intervention on the level of perceived loudness of the tinnitus (Andersson 1999; Martinez-Devesa 2010). Additionally, concerns have been raised about what is actually being measured when people are asked to rate the subjective loudness of their tinnitus (McKenna 2014).

Clinical management strategies include education and/or counselling, relaxation therapy, tinnitus retraining therapy (TRT), cognitive behavioural therapies (CBT) and sound enrichment using ear-level sound generators or hearing aids (Henry 2005). In addition, electrical and neurostimulation, as well as drug therapies aimed at treating tinnitus directly, or managing co-morbid symptoms such as insomnia, anxiety or depression, have been tested. The effects of these management options are variable, they have inconclusive outcomes and some have risks or adverse effects (Dobie 1999; Hoare 2011a; Hoare 2011b; Hobson 2012; Langguth 2013; Martinez-Devesa 2010; Phillips 2010).

\section{Description of the intervention}

Cognitive behavioural therapy (CBT) is an inclusive term that features and combines numerous psychological interventions that were developed and evolved from cognitive and behavioural therapies respectively. CBT for tinnitus aims primarily to reduce the impact of tinnitus on quality of life, rather than directly change the perceived loudness.

Behavioural therapies (e.g. behavioural activation, exposure, relaxation) aim to help patients overrule learned associations between tinnitus and counter-productive responses (e.g. avoiding tinnitus-increasing activities). Cognitive therapies, on the other hand, focus on the relationship between thoughts and emotions (Ellis 1977), and apply a process of identification and modification of errors in thought processing of experiences (Beck 1979). Combined, the behavioural and cognitive theories have produced a range 
of intervention components designed to address the dysfunctional thought processes, behavioural and emotional responses that maintain low tinnitus-related quality of life.

As discussed by Cima 2014, cognitive behavioural interventions such as mindfulnessbased stress reduction (also known as 'mindfulness'; Kabat-Zinn 1982) and acceptance and commitment therapy (ACT; Hayes 1999) have been developed and applied to the treatment of the impact of tinnitus on quality of life (e.g. Hesser 2009; Philippot 2012). For the purposes of this review, we will not make distinctions between whether an intervention is 'first', 'second' or 'third wave' CBT. Instead, we will treat ACT and mindfulness interventions as CBT and in the course of data extraction we will identify components/ elements within all interventions as behavioural, cognitive or a combination (i.e. CBT).

Interventions described or labelled as 'CBT' cannot be assumed to be equivalent homogenous entities. Even if CBT interventions comprise the same elements they might vary with regard to: the mode of delivery of the intervention (e.g. face-toface, mediated via telephone, Internet); the frequency of sessions (e.g. daily, weekly, fortnightly); the length of sessions; the duration of the intervention; who delivers the CBT (e.g. psychologist, social worker, nurse, computer program); the setting in which the treatment is delivered (e.g. hospital, health centre, private clinic); and whether the therapy is delivered in a group or individual format.

The previous Cochrane Review of CBT for tinnitus found that there were no reported adverse effects in the included studies (Martinez-Devesa 2010). It is, however, conceivable that people might experience a deterioration in their mood during the course of CBT, due to the often challenging nature of the therapy or the distress arising as a result of changes in cognitive and emotional mechanisms. It is also possible that adverse effects were not reported by the authors of studies included in the review, as this is a common occurrence in studies (Pitrou 2009).

\section{How the intervention might work}

Since a growing body of evidence suggests that the impact of tinnitus on quality of life depends more on psychological factors than acoustic properties (Cima 2014; Milerova 2013), psychological therapies have been widely used for tinnitus treatment.

Cognitive strategies are based on the idea that negatively biased interpretations or thoughts about specific events or experiences, such as hearing tinnitus, produce a dysfunctional emotional and/or behavioural response (Beck 1979; Ellis 1977). Thus, cognitive strategies are thought to work by identifying any biased or irrational thinking styles (such as catastrophizing), then challenging, modifying and/or replacing them with alternative and more realistic beliefs that lead to a more adaptive response.

A behavioural intervention such as an exposure therapy might be utilised to decrease the impact of tinnitus on daily life. Exposure to the tinnitus sound is thought to work through a process of extinction learning and generalisation. That is, a person learns that the tinnitus sound is no longer indicative of being emotionally aroused or in a distressed state and applies this new knowledge to situations beyond those learned in 
the therapeutic setting. In daily life this might mean a person re-engages in activities that they previously avoided for fear that the tinnitus would deteriorate.

Individually, cognitive and behavioural therapy components are hypothesised to have specific effects. For example, education regarding the physiology and pathophysiology of hearing and tinnitus are thought to provide a foundation on which patients can begin to understand that tinnitus is not a harmful symptom in its own right and hence nothing, logically at least, to be afraid of. Cognitive behavioural approaches to tinnitus therapy are therefore hypothesised to affect a reduction in impact of tinnitus on quality of life through the summed or synergistic effects of the specific intervention components included in an individual therapy. Further, it is hypothesised that this has a consequent effect of reducing generalised anxiety or depression where it is co-morbid, and generally improving self-reported quality of life.

To date there has been little detailed research examining precisely when therapeutic change occurs during the course of CBT treatments, but they have been reported to be effective over at least a 12-month period (e.g. Cima 2012).

\section{Why it is important to do this review}

This review includes recent randomised controlled trials of CBT for tinnitus that were not included in previous meta-analyses or recent reviews. The most recently published review of CBT interventions for tinnitus was a historical and narrative overview in which a range of study designs in addition to RCTs were included, but also one in which neither a risk of bias assessment was undertaken nor a meta-analysis conducted (Cima 2014). These methodological issues make it harder to draw conclusions about the strength of any treatment effects and risks of bias in the evidence included in the narrative synthesis.

A second reason is that it was also important to address new questions that will inform decisions about service provision, as this has particular relevance for the policy-makers and agencies involved in the funding of treatment (e.g. insurance companies). CBT for tinnitus is generally well received by patients and is potentially a cost-effective means for reducing the impact of tinnitus on quality of life (Maes 2014), but it would also be informative to compare the effectiveness of CBT delivered in group and individual formats and CBT performed by psychologists compared with other health professionals.

Finally, since the previous version of the Cochrane Review of CBT for tinnitus was published (Martinez-Devesa 2010), Cochrane standards for the conduct of intervention reviews have been revised (Higgins 2013; Higgins 2016). This new review not only includes recent randomised controlled trials, but also complies with the new standards.

In summary, this review synthesises the latest evidence related to CBT for tinnitus, which will help inform decisions on whether CBT for tinnitus is effective at reducing the impact of tinnitus on quality of life.

\section{Objectives}

To assess the effects and safety of CBT for tinnitus in adults. 


\section{Methods}

\section{Criteria for considering studies for this review}

\section{Types of studies}

Randomised controlled trials (including cluster-randomised). If included studies had used a cross-over design, we would have only included data from the first treatment phase. Quasi-randomised controlled studies were not included.

We did not apply restrictions on language, year of publication or publication status.

\section{Types of participants}

Participants were at least 18 years of age with tinnitus as the primary reason for seeking treatment.

In the event that studies included an age range of participants below 18 years (e.g. 16 to 21 years), they were included if the mean age was 18 years or above.

\section{Types of interventions}

The primary intervention of interest was CBT. For the purposes of this review we included studies that also described CBT interventions that apparently only used cognitive or behavioural elements. Interventions such as acceptance and commitment therapy (ACT) and mindfulness were also included but simply considered as types of CBT.

We considered interventions as 'mindfulness' if they involved: exercises that involved self-regulation of attention on experience and emphasised openness, curiosity and acceptance (Bishop 2004).

For the purposes of determining similarities for subgroup analysis, we would have attempted to contact authors of studies that examined the effectiveness of an apparently 'pure' cognitive or behavioural interventions to obtain treatment manuals or protocols.

Upon receipt of any protocols, two authors would have then independently reviewed the intervention manual classifying treatment elements as either cognitive or behavioural. Based on results from a review of treatment components used in psychological therapy for people with tinnitus (Thompson 2016) and the behaviour change taxonomy (Michie 2013), we classified interventions as either 'cognitive only', 'behavioural only' or 'CBT'. In the event that the review authors had differed in their judgements, a third review author would have acted as an arbiter.

We stratified studies into four comparisons:

1. CBT versus no intervention/waiting list control;

2. CBT versus usual audiological care (tinnitus education and rehabilitation for hearing loss); 
3. CBT versus TRT (directive counselling and the use of bilateral sound generators as per TRT protocol);

4. CBT versus other experimental control (pooled if using the same experimental control). Other experimental controls may include transcranial magnetic stimulation, electrical or electromagnetic stimulation therapy and bio- neuro-feedback.

\section{Types of outcome measures}

We analysed the following outcomes in the review, but did not use them as a basis for including or excluding studies.

\section{Primary outcomes}

Impact of tinnitus on quality of life as measured by validated tinnitus-specific multi-item questionnaires identified in a systematic review of outcome instruments used in studies of interventions for tinnitus (Hall 2016). These included:

- Tinnitus Functional Index;

- Tinnitus Handicap Inventory;

- Tinnitus Handicap Questionnaire;

- Tinnitus Questionnaire;

- Tinnitus Reaction Questionnaire;

- Tinnitus Disability Index;

- Tinnitus Severity Scale.

For references associated with the outcome measures see Appendix 4).

If a study used multiple measures of the impact of tinnitus on quality of life we applied the following as a hierarchy of the outcome measures based on their known psychometric validity (Fackrell 2014): Tinnitus Functional Index, Tinnitus Handicap Inventory, Tinnitus Handicap Questionnaire, Tinnitus Questionnaire, Tinnitus Reaction Questionnaire, Tinnitus Disability Index, Tinnitus Severity Scale and then other tinnitus-specific questionnaires. Invariably these questionnaires show good convergent validity.

Serious adverse effects: self-harm, suicide, suicide attempt, suicidal crisis, severe symptom exacerbation.

\section{Secondary outcomes}

- Generalised depression as measured by validated questionnaires, such as the Beck Depression Inventory II (Beck 1996), the depression scale of the Hospital Anxiety and Depression Scale (HADS; Zigmond 1983), and the Hamilton Rating Scale for Depression (Hamilton 1960).

- Generalised anxiety as measured by a validated scale, for example, the anxiety scale of the HADS or Beck Anxiety Inventory (Beck 1988) or the Anxiety Sensitivity Index (Reiss 1986).

- Health-related quality of life as measured by a validated scale, for example, the Short-Form 36 (Hays 1993), WHOQoL-BREF (Skevington 2004), and other 
WHOQoL versions, Health Utilities Index (Furlong 2001).

- Negatively biased interpretations of tinnitus as measured by a validated scale, such as the Tinnitus Catastrophizing Scale (Cima 2011b), the Fear of Tinnitus Questionnaire (Cima 2011b), and the Tinnitus Fear and Avoidance Scale (Kleinstauber 2013).

- Other adverse effects: acute emotional discomfort.

We measured outcomes at treatment end (typically six to eight weeks) and at long-term follow-up (6 and 12 months).

\section{Search methods for identification of studies}

The Cochrane ENT Information Specialist conducted systematic searches for randomised controlled trials and controlled clinical trials. There were no language, publication year or publication status restrictions. The date of the search was 25 November 2019.

\section{Electronic searches}

The Information Specialist searched:

- the Cochrane ENT Trials Register (searched 25 November 2019);

- CENTRAL (2019, Issue 11) via the Cochrane Register of Studies (25 November 2019);

- Epub Ahead of Print, In-Process \& Other Non-Indexed Citations, Ovid MEDLINE(R) Daily and Ovid MEDLINE(R) 1946 to 25 November 2019);

- Ovid EMBASE (1974 to 25 November 2019);

- $\quad$ EBSCO CINAHL (1982 to 25 November 2019);

- Ovid AMED (1985 to 25 November 2019);

- Ovid PsycINFO (1806 to 25 November 2019);

- Web of Knowledge, Core Collection (1945 to 25 November 2019);

- ClinicalTrials.gov, (searched via the Cochrane Register of Studies 26 November 2019);

- World Health Organization (WHO) International Clinical Trials Registry Platform (ICTRP), www.who.int/ictrp (searched via the Cochrane Register of Studies 26 November 2019).

In searches prior to November 2019, we also searched LILACS, KoreaMed, IndMed and PakMediNet to November 2018.

The Information Specialist modelled subject strategies for databases on the search strategy designed for CENTRAL. Where appropriate, they were combined with subject strategy adaptations of the highly sensitive search strategy designed by Cochrane for identifying randomised controlled trials and controlled clinical trials (as described in the Cochrane Handbook for Systematic Reviews of Interventions Version 5.1.0, Box 6.4.b. (Higgins 2011). Search strategies for major databases including CENTRAL are provided in Appendix 5.

\section{Searching other resources}

We scanned the reference lists of identified publications for additional studies and contacted study authors where necessary. In addition, the Information Specialist 
searched Ovid MEDLINE to retrieve existing systematic reviews relevant to this systematic review, so that we could scan their reference lists for additional studies. The Information Specialist also ran non-systematic searches of Google Scholar to retrieve grey literature and other sources of potential studies.

\section{Data collection and analysis}

\section{Selection of studies}

Thomas Fuller (TF) and Rilana Cima (RC) independently screened titles and abstracts from the search results for eligible studies. When there were disagreements at the screening stage, we obtained copies of the full-text articles and examined them closely for eligibility. For all disagreements over full-text articles being assessed for inclusion, a third review author was consulted as an arbiter.

We recorded and presented the flow of study identification and selection in the form of a PRISMA flow chart (Moher 2009; Figure 1).

\section{Data extraction and management}

TF co-ordinated the retrieval of full-text articles as well as the management and extraction of all data. Two of TF, Derek Hoare (DH), RC or Birgit Mazurek (BM) independently extracted data from the included studies into standardised data forms based on a generic form developed by the Cochrane ENT editorial group. In the event that one of the review authors was the author of an included study he or she did not extract data from the study. Where relevant, the review authors copied and pasted verbatim text from included studies into the data extraction form. Any disagreements in the data extraction were initially addressed through discussion between the review authors involved. If that did not lead to agreement, a third review author was consulted as an arbiter. In the event of information not being reported in adequate detail to enable decisions about inclusion or exclusion, we contacted (or at least attempted multiple times to do so) study authors to request the provision of additional information.

Data extraction included information on the following: details of the source of participants, eligibility criteria, methods, participants, intervention treatment elements, outcome measures at baseline (or pre-test) and other time points reported in the respective studies, results including estimates of effects and confidence intervals, details of the funding source, key conclusions from the authors, comments from the review authors especially with regard to any differences between protocols and study reports, details of any correspondence required and any references to other relevant studies. Further details of the data to be extracted for intervention reviews are specified in table 7.2 of the Cochrane Handbook for Systematic of Interventions (Higgins 2011).

At the completion of data collection and once there was agreement on the data set that had been extracted, we entered the data into Review Manager 5.3 (RevMan 2014). 


\section{Assessment of risk of bias in included studies}

$\mathrm{TF}, \mathrm{BM}, \mathrm{DH}$ and $\mathrm{RC}$ completed assessment of the risk of bias of the included studies independently, with the following taken into consideration, as guided by the Cochrane Handbook for Systematic Reviews of Interventions (Higgins 2011):

- sequence generation;

- allocation concealment;

- blinding;

- incomplete outcome data;

- selective outcome reporting; and

- other sources of bias.

We used the Cochrane 'Risk of bias' tool in RevMan 5.3 (RevMan 2014), which involved describing each of these domains as reported in the study and then assigning a judgement about the adequacy of each entry: 'low', 'high' or 'unclear' risk of bias. In the event of disagreement between assessors of risk of bias, we discussed the rationale for the respective judgements in an effort to resolve the differences. If that did not lead to agreement, a third review author acted as an arbiter.

\section{Measures of treatment effect}

We analysed ordinal data as if it were continuous data and used standardised mean differences (SMD) and Cohen's d effect size measurement to estimate treatment effects for measures of the impact of tinnitus on quality of life and other continuous measures of secondary outcomes. If feasible, we also pooled data from the same scale and used mean differences (MD).

We analysed dichotomous data using risk ratios (RR) and reported all results with $95 \%$ confidence intervals (95\% Cls).

\section{Unit of analysis issues}

One study used a cluster-randomised design so we chose statistical methods in consultation with a statistician and following the recommendations in the Cochrane Handbook for Systematic Reviews of Interventions to "extract an estimate of the required effect measure from an analysis that accounts for the cluster design" using an odds ratio with confidence interval or generalised estimating equations (Higgins 2011). Also as specified we used the inverse variance method to meta-analyse effect estimates and standard errors so that the clustered nature of the data was taken into consideration (Higgins 2011).

We did not include any RCTs that used a cross-over design. Had we done so, individual participant data constituting the unit of analysis from the first treatment phase would have been included in the meta-analysis.

\section{Dealing with missing data}

Whenever possible we attempted to contact investigators to request missing data relating to, for example, study characteristics, outcome measures and how many 
patients dropped out or were included in the analysis. In relation to missing information about dropout or numbers included in the analysis, if we did not receive a response or data from the authors, we conducted the analysis using a conservative approach and assumed that the missing participants' data indicated no effect of/from the intervention. We undertook a sensitivity analysis to examine the effect of this assumption by comparing the results with what would happen if the missing participants had the best possible outcome.

In one study standard deviations were not reported for the Tinnitus Effects Questionnaire (TEQ) total score (Jakes 1992). It was not possible, from the information reported in Jakes 1992, to estimate the standard deviations, so we made a decision to use the standard deviation reported in Henry 1998a.

Where there were missing standard deviations for continuous data, we used methods to estimate these using confidence intervals, standard errors, t, P or F values where reported.

We report the attempts to contact authors for missing data and responses (or otherwise), along with consideration of the potential impact of the missing data, in the Discussion of the review.

\section{Assessment of heterogeneity}

We investigated clinical heterogeneity with regard to: components of the interventions, mode of delivery, level of action, who delivered the CBT and the type of intervention used in the control condition. We also assessed methodological heterogeneity according to study design and risk of bias (i.e. randomisation, blinding of outcome assessment, and losses to follow-up).

We assessed the degree of statistical heterogeneity that existed across studies using the 12 statistic and we used the following from the Cochrane Handbook for Systematic Reviews of Interventions as a guide for interpretation (Higgins 2011):

- $0 \%$ to $40 \%$ : might not be important;

- $30 \%$ to $60 \%$ : may represent moderate heterogeneity;

- $50 \%$ to $90 \%$ : may represent substantial heterogeneity;

- $75 \%$ to $100 \%$ : considerable heterogeneity.

\section{Assessment of reporting biases}

We examined reporting bias through the creation of funnel plots for the comparisons of CBT versus no intervention/wait list control and CBT versus any other active comparator.

\section{Data synthesis}

We conducted meta-analyses using random-effects models as we expected that there would be differences between the study populations and methods used. We conducted sensitivity analyses using fixed-effect models. 
We pooled studies where there was sufficient similarity between them with regard to: outcome (good convergent validity), level of action (i.e. individual or group therapy) and mode of delivery (i.e. in person, face-to-face or online).

We stratified studies into four comparisons:

- CBT versus no intervention/waiting list control;

- $\quad$ CBT versus usual audiological care (tinnitus education and rehabilitation for hearing loss);

- $\quad$ CBT versus TRT (directive counselling and bilateral masking);

- CBT versus other experimental control (pooled if using the same experimental control). Other experimental controls may include transcranial magnetic stimulation, electrical or electromagnetic stimulation therapy or bio- neuro-feedback.

The intention was to pool the results of the CBT treatments. While CBT treatment protocols differed we judged them, within the particular sub-types of CBT, to be similar enough to conduct meta-analyses although there was significant statistical heterogeneity.

\section{Subgroup analysis and investigation of heterogeneity}

We conducted the following subgroup analyses for the primary outcome of the impact of tinnitus on quality of life:

- Studies by types of therapy: 'cognitive only', 'behavioural only', 'cognitive and behavioural only', ACT, mindfulness.

- Studies by modes of delivery: 'face-to-face' or 'online CBT'.

- Studies by unit of delivery: ‘individual patient therapy' or 'group therapy'.

- Study or patient groups by who delivers CBT; 'psychologists' or 'psychiatrists' or 'audiologists' or other therapists or clinicians.

- Studies by whether participants are included/excluded according to their hearing status: 'hearing loss was an exclusion criterion' or 'hearing loss was not an exclusion criterion'.

\section{Sensitivity analysis}

We conducted the following sensitivity analyses to examine the role of:

- meta-analysis using random-effects and fixed-effect models respectively;

- including or excluding studies at high risk of bias for incomplete outcome data.

- replacing missing data with a conservative compared with an 'optimistic' approach in the event that data within a particular study were not collected (or reported) at the end of treatment.

\section{Summary of findings and assessment of the certainty of the evidence}

We used the GRADE approach to rate the overall certainty of evidence. The certainty of evidence reflected the extent to which we were confident that an estimate of effect was correct and we applied this to the interpretation of results. There were four possible 
ratings: high, moderate, low, and very low. A rating of high certainty of evidence implied that we were confident in our estimate of effect and that further research is very unlikely to change our confidence in the estimate of effect. A rating of very low certainty implied that any estimate of effect obtained is very uncertain.

The GRADE approach rates evidence from RCTs that do not have serious limitations as high certainty. However, several factors can lead to the downgrading of the evidence to moderate, low, or very low. The degree of downgrading is determined by the seriousness of these factors:

- study limitations (risk of bias);

- inconsistency;

- indirectness of evidence;

- imprecision; and

- publication bias.

'Summary of findings' tables for CBT compared with no intervention/waiting list control, usual audiological care, TRT and other control interventions are presented (Summary of findings table 1; Summary of findings table 2; Summary of findings table 3; Summary of findings table 4). The tables include the following outcomes: impact of tinnitus on quality of life, serious adverse effects, depression, anxiety, health-related quality of life, negatively biased interpretations of tinnitus and other adverse effects.

\section{Results}

\section{Description of studies}

\section{Results of the search}

The Cochrane ENT Information Specialist conducted an electronic search of the literature in November 2019. A total of 3180 records were identified through this method, of which 1350 remained after duplicates were removed. We excluded 1148 references on the basis of title or abstract and retrieved a total of 102 records for full-text review. We discarded 54 records and excluded 20 (10 because the allocation of participants was not randomised, nine because the interventions were not CBT and one because there was not a relevant comparator). See Characteristics of excluded studies for details. Five records were for ongoing studies (see Characteristics of ongoing studies). There are no studies awaiting assessment.

In total we included 28 studies in this review. Twenty-two of these studies reported quantitative data, which were included in meta-analyses (Abbott 2009; Andersson 2002; Andersson 2005; Arif 2017; Beukes 2018a; Beukes 2018b; Cima 2012; Davies 1995; Henry 1996; Hesser 2012; Jakes 1992; Jasper 2014; Kaldo 2007; Kreuzer 2012; Malinvaud 2016; McKenna 2017; Nyenhuis 2013a; Oron (unpublished); Philippot 2012a; Schmidt 2018; Weise 2016; Westin 2011). Six studies did not present usable data (Henry 1998; Jakes 1986; Lindberg 1989; Martz 2018; Robinson 2008; Zhong 2014). 
No additional studies were identified through other search methods, which included contacting researchers and hand searching the references of included studies.

Figure 1 presents the study retrieval and selection process, and reasons for exclusion.

Figure 1. Process for Sifting Search Results and Selecting Studies for Inclusion.

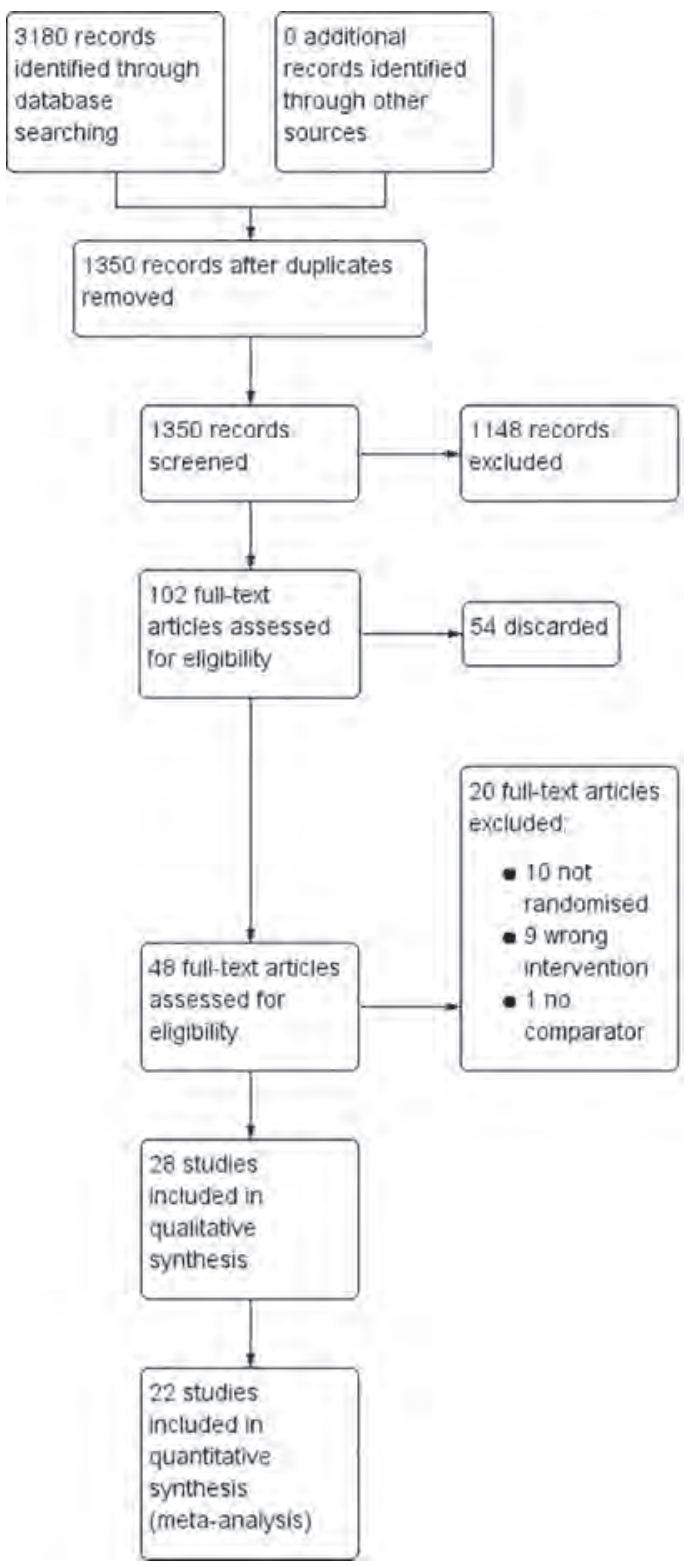




\section{Included studies}

We included 27 published studies and one unpublished study that is being prepared for publication (Oron (unpublished)). For descriptions of the studies, see the Characteristics of included studies table.

\section{Design}

Twenty-seven studies were parallel-group RCTs and one was a cluster-RCT (Abbott 2009).

Three studies had multiple intervention/treatment arms (Jasper 2014; Hesser 2012; Martz 2018). Jasper 2014 was a three-arm trial in which Internet-based CBT (iCBT) and group-CBT was compared with an Internet-based discussion forum. Hesser 2012 was also a three-arm trial but compared a CBT intervention with an ACT intervention and an online discussion forum condition respectively. Martz 2018 examined the efficacy of CBT, ACT, Coping Effectiveness Training and a wait list control condition.

\section{Sample sizes}

The total sample size for all included studies was 2733. Within studies the sample size ranged from 23 (Andersson 2005) to 492 (Cima 2012) participants.

\section{Setting}

Nine studies were set in hospitals. Two of these were in England (Davies 1995; McKenna 2017), two in Sweden (Lindberg 1989; Westin 2011), and one each in China (Zhong 2014), France (Malinvaud 2016), Israel (Oron (unpublished)), the Netherlands (Cima 2012), and Wales (Arif 2017). A total of five studies, in four countries, were conducted online (i.e. using Internet-based interventions): two in Sweden (Andersson 2002; Hesser 2012), and one in Australia (Abbott 2009), England (Beukes 2018a), and Germany (Weise 2016) respectively. In one study the intervention (iCBT) was conducted online while the comparator, audiological treatment as usual was conducted in hospital settings (Beukes 2018b).

Three studies were conducted in Veterans Affairs clinics in the USA (Martz 2018; Robinson 2008; Schmidt 2018) and two studies were conducted in psychology clinics (one in Belgium (Philippot 2012a) and one in Sweden (Andersson 2005)). Nyenhuis 2013a conducted a study with four arms that included an online condition, a bibliotherapy condition and interventions delivered face-to-face in two "study centres" in the southern region of Lower Saxony, Germany. One study, set in Sweden, delivered the intervention primarily through bibliotherapy (Kaldo 2007). Two of three conditions in Jasper 2014 (set in Germany) were delivered as an Internet-based intervention (i.e. iCBT and the control condition), while the setting for group CBT was not described. Five studies did not report the setting in which the studies were conducted; two were from Australia (Henry 1996; Henry 1998), two were from England (Jakes 1986; Jakes 1992), and one was from Germany (Kreuzer 2012).

Of the 28 included studies, six were from England, six from Sweden, four from Germany, three from the USA, three from Australia, and one each from Belgium, China, France, Israel, the Netherlands and Wales. 


\section{Participants}

All studies included adult participants (18 years or over) with the mean age of participants ranging from 42.6 years to 70.1 years. Six studies limited the maximum age of participants: one limited it to 65 years (Abbott 2009); three to 70 years (Andersson 2002; Jakes 1986; Malinvaud 2016); one to 75 years (Nyenhuis 2013a); and one to 80 years (Kreuzer 2012). One study had a minimum age of 65 years (Andersson 2005). Eleven studies did not report inclusion or exclusion criteria related to age (Davies 1995; Henry 1996; Henry 1998; Jakes 1992; Jasper 2014; Lindberg 1989; Martz 2018; Oron (unpublished); Philippot 2012a; Robinson 2008; Zhong 2014).

Of all the participants in the included studies, $40.7 \%$ were female $(n=1106)$ and $58.1 \%$ were male $(n=1579)$. There were missing data on gender for 34 participants from five studies (Abbott 2009; Davies 1995; Henry 1996; Malinvaud 2016; Schmidt 2018), although one study accounted for approximately $44 \%$ of this $(n=15)$ (Davies 1995). Three of the 28 studies had a greater proportion of female than male participants (53.5\%, 52.4\% and 59.7\% respectively) (Arif 2017; Jakes 1992; Weise 2016). The proportion of males in the included studies ranged from $28.9 \%$ (Davies 1995) to $82.1 \%$ (Abbott 2009).

The reported tinnitus duration ranged from a minimum average of 3.2 months (Nyenhuis 2013a) to a maximum average of 22.9 years (Schmidt 2018). A minimum tinnitus duration was not required/reported in nine studies (Arif 2017; Beukes 2018b; Cima 2012; Jakes 1986; Martz 2018; Oron (unpublished); Philippot 2012a; Robinson 2008; Zhong 2014), although three did require a referral and/or diagnosis from a medical professional such as an Ear, Nose and Throat surgeon or general practitioner (Beukes 2018b; Cima 2012; Philippot 2012a). Seven studies specified that participants had a diagnosis from a medical professional as part of their inclusion criteria (Abbott 2009; Andersson 2002; Henry 1996; Henry 1998; Hesser 2012; Kaldo 2007; Weise 2016).

Most studies (24 of the 28) stated or described in their inclusion criteria a level of tinnitus severity required to participate. Ten studies gave cut-off scores on self-report questionnaires as criteria indicating minimum levels of severity. Within this group, there was some variation on the specific cut-off scores and questionnaires referred to. Specifically, Jasper 2014 required participants to have a minimum score of 18 on the THI, compared with others who required minimums of 20 (Schmidt 2018), 30 (Westin 2011) or 38 (Hesser 2012; Weise 2016). Jasper 2014 and Weise 2016 also specified additional cut-off scores on the mini-TQ (8 and 13 respectively) and Schmidt 2018 specified a minimum score of 17 on the $\mathrm{TRQ}$, and 5 or more on the Tinnitus Impact Screening Interview. Three studies referred solely to a TRQ score, though there was also a difference in cut-offs (Henry 1996; Henry 1998; Kaldo 2007); 10 or more for Kaldo 2007, and 17 or more for Henry 1996 and Henry 1998. Martz 2018 required prospective participants to have a minimum score of 21 and Beukes 2018a required participants to have a minimum of 25 on the Tinnitus Functional Index (TFI). Descriptions and/or indicators of tinnitus severity referred to in other studies included for example: "primary complaint of tinnitus" (e.g. Cima 2012), "self-reported distress due to tinnitus" (Robinson 
2008), and "significant psychological distress and impairment in everyday activities resulting from tinnitus" (Philippot 2012a).

One study excluded participants with severe hearing loss due to the impact this could have on the use of wearable sound generators (Westin 2011), but otherwise hearing loss was not applied as an exclusion criterion for participating in the studies.

In relation to co-morbid psychological conditions, 16 studies included measures of anxiety and 23 included measures of depression. However, only three studies specifically referred to anxiety or depression in their inclusion or exclusion criteria (Andersson 2005; Kaldo 2007; Weise 2016). Kaldo 2007 specified that participants must have scores lower than 19 on both the anxiety and depression subscales of the Hospital Anxiety and Depression Scale. Andersson 2005 included people with scores lower than 22 on the Beck Depression Inventory, and Weise 2016 included those without a "clinical diagnosis of depression". It was, however, more common (15 out of 28 studies) that descriptive criteria about psychopathology were used to exclude potential participants. For example, criteria would refer to prospective participants with/without the presence/ absence of a major psychiatric condition or disorder (Beukes 2018a; Davies 1995; Jakes 1992). Five studies also specified high risk of suicide in their exclusion criteria (Andersson 2005; Hesser 2012; Jasper 2014; McKenna 2017; Weise 2016). Other specific psychological conditions referred to in participant selection criteria included substance use disorders (McKenna 2017; Schmidt 2018; Weise 2016), psychosis (Robinson 2008; Schmidt 2018), and personality disorders (Philippot 2012a).

\section{Interventions and comparisons}

Cognitive, behavioural, ACT, mindfulness, and cognitive and behavioural (combined) interventions were considered as 'CBT' and thus eligible for inclusion in this review. (Note that in the following description of the studies, some had more than one CBT and/or control arm within the study, and thus the total number of comparisons does not equal 28). Seventeen studies tested CBT (Abbott 2009; Andersson 2002; Andersson 2005; Beukes 2018a; Beukes 2018b; Cima 2012; Jasper 2014; Kaldo 2007; Lindberg 1989; Malinvaud 2016; Martz 2018; Nyenhuis 2013a; Robinson 2008; Schmidt 2018; Zhong 2014); five tested cognitive interventions (Davies 1995; Henry 1996; Henry 1998; Jakes 1986; Jakes 1992), four tested ACT (Hesser 2012; Martz 2018; Oron (unpublished); Westin 2011), and four tested mindfulness interventions. Within the mindfulness interventions, two tested mindfulness meditation (Arif 2017; Kreuzer 2012), one tested a mindfulness-based stress reduction (Philippot 2012a), and one tested a mindfulness-based cognitive therapy intervention (McKenna 2017). No studies tested purely behavioural interventions.

The most common mode by which interventions were delivered was face-to-face. Twenty-one studies delivered CBT face-to-face (Andersson 2005; Arif 2017; Cima 2012; Davies 1995; Henry 1996; Henry 1998; Jakes 1986; Jakes 1992; Jasper 2014; Kreuzer 2012; Lindberg 1989; Malinvaud 2016; Martz 2018; McKenna 2017; Nyenhuis 2013a; Oron (unpublished); Philippot 2012a; Robinson 2008; Schmidt 2018; Westin 2011; Zhong 2014), six delivered CBT in the form of an Internet-based intervention (Abbott 2009; Andersson 
2002; Beukes 2018a; Beukes 2018b; Hesser 2012; Weise 2016), and one multi-arm study included an Internet-based and face-to-face CBT condition (Jasper 2014). Kaldo 2007 compared CBT delivered as bibliotherapy with email contact with a wait list control condition. Seventeen studies delivered CBT in a group format (Andersson 2005; Cima 2012; Henry 1996; Henry 1998; Jakes 1986; Jakes 1992; Jasper 2014; Kreuzer 2012; Lindberg 1989; Malinvaud 2016; Martz 2018; McKenna 2017; Nyenhuis 2013a; Oron (unpublished); Philippot 2012a; Robinson 2008; Schmidt 2018; Zhong 2014), 10 studies delivered CBT individually (Abbott 2009; Andersson 2002; Arif 2017; Beukes 2018a; Beukes 2018b; Davies 1995; Jakes 1986; Kaldo 2007; Weise 2016; Westin 2011), and one study included an individual and group CBT condition (Jasper 2014).

Professions involved in delivering interventions included psychologists (Abbott 2009; Andersson 2005; Davies 1995; Henry 1996; Hesser 2012; Jasper 2014; Kaldo 2007; Lindberg 1989; Malinvaud 2016; Martz 2018; McKenna 2017; Nyenhuis 2013a; Philippot 2012a; Robinson 2008; Schmidt 2018; Weise 2016; Westin 2011), and audiologists (Beukes 2018a; Beukes 2018b). In one study a multidisciplinary team delivered CBT (Cima 2012), and in another psychologists and psychiatrists delivered the intervention (Robinson 2008). Three studies described the people delivering the interventions as "therapists" without providing details of qualifications (Arif 2017; Jakes 1992; Kreuzer 2012), and four studies did not report any information about who delivered the intervention (Henry 1998; Jakes 1986; Oron (unpublished); Zhong 2014).

\section{CBT versus no intervention/waiting list control}

Fourteen studies compared CBT to wait list control conditions (Andersson 2002; Andersson 2005; Beukes 2018a; Henry 1996; Henry 1998; Jakes 1992; Kaldo 2007; Kreuzer 2012; Lindberg 1989; Malinvaud 2016; Martz 2018; Oron (unpublished); Robinson 2008; Westin 2011). The duration of the waiting list control period ranged from 3 (Lindberg 1989) to 22 (Kreuzer 2012) weeks, with the median being 6 weeks, and the average waiting period being 8.1 weeks. In all studies, participants were offered the CBT intervention at the end of the waiting period.

\section{CBT versus usual audiological care (tinnitus education and rehabilitation for hearing loss)}

Three studies compared CBT to audiological care (Beukes 2018b; Cima 2012; Schmidt 2018). Beukes $2018 \mathrm{~b}$ compared an individually delivered, eight-week iCBT (with optional email contact with an audiologist) intervention to audiological care as usually delivered in the UK; that is, three 60-minute appointments, and two follow-up appointments at one and two months respectively. In Cima 2012, the CBT intervention was delivered faceto-face, according to a stepped-care model where those requiring greater assistance received a greater number of sessions. The audiological care condition in Cima 2012 was based on the results from a survey of audiologists asking what care they provided to patients with tinnitus, as at the time there was no standardised audiological care for tinnitus in the Netherlands. The audiological care in Cima 2012 also comprised a stepped-care approach where patients first had audiological tests and education in step 1, and then if needed in step 2, up to nine sessions with a social worker. Schmidt 2018 tested a six-week face-to-face group CBT intervention developed specifically for 
veterans of military service. Audiological care was also delivered in groups over six weeks, and included tinnitus education and attentional skills training (Schmidt 2018).

\section{CBT versus tinnitus retraining therapy}

One study compared CBT to TRT and a wait list control condition (Westin 2011). The CBT intervention comprised Acceptance and Commitment Therapy (ACT) delivered individually over the course of 10 weeks in 60- to 75-minute sessions. TRT involved a 2.5-hour consultation with an ENT physician which included a diagnostic assessment, and directive counselling. Participants in the TRT condition were also fitted with bilateral sound generators (as per TRT protocol) and instructed to use them for a minimum of eight hours per day over an 18-month period.

\section{CBT versus other active control}

Sixteen studies compared CBT to an active experimental control group not otherwise included in the previous comparisons (Abbott 2009; Arif 2017; Davies 1995; Henry 1996; Hesser 2012; Jakes 1986; Jakes 1992; Jasper 2014; Malinvaud 2016; Martz 2018; McKenna 2017; Nyenhuis 2013a; Oron (unpublished); Philippot 2012a; Weise 2016; Zhong 2014).

The CBT interventions included: CBT (Abbott 2009; Jakes 1986; Jasper 2014; Malinvaud 2016; Martz 2018; Nyenhuis 2013a; Weise 2016; Zhong 2014), cognitive therapy (Davies 1995; Henry 1998; Jakes 1992), ACT (Hesser 2012; Martz 2018; Oron (unpublished), and mindfulness (Arif 2017; McKenna 2017; Philippot 2012a). Eleven CBT interventions were delivered face-to-face (Arif 2017; Davies 1995; Henry 1996; Jakes 1986; Jakes 1992; Malinvaud 2016; Martz 2018; McKenna 2017;Nyenhuis 2013a; Oron (unpublished); Zhong 2014), and three were delivered as Internet-based interventions (Abbott 2009; Hesser 2012; Weise 2016). Jasper 2014 included a group CBT and iCBT arm in addition to a control condition.

CBT was provided individually in five studies (Abbott 2009; Arif 2017; Davies 1995; Hesser 2012; Weise 2016), in groups in nine studies (Henry 1996; Jakes 1986; Jakes 1992; Malinvaud 2016; Martz 2018; McKenna 2017; Nyenhuis 2013a; Oron (unpublished); Philippot 2012a), and both individually and in groups in Jasper 2014. In one study, no information was reported to indicate whether participants engaged in treatment individually or in groups (Zhong 2014).

In nine studies psychologists delivered CBT (Davies 1995; Henry 1996; Jakes 1986; Jasper 2014; Malinvaud 2016; Martz 2018; McKenna 2017; Nyenhuis 2013a; Philippot 2012a), four studies tested guided Internet-based interventions by psychologists who were available via email to answer questions and provide feedback (Abbott 2009; Hesser 2012; Jasper 2014; Weise 2016), and two studies reported that "therapists" delivered the intervention but did not provide further information on their qualifications or experience (Arif 2017; Jakes 1992). One study, Zhong 2014, did not report what professional delivered the CBT. 
Other experimental control interventions included:

- Relaxation (Arif 2017; Davies 1995; Jakes 1986; McKenna 2017; Philippot 2012a). The types of relaxation used as active control conditions included: applied relaxation based on the work of Bernstein 1973 (Jakes 1986), Bernstein 1984 (Davies 1995), Jacobson 1957 (Philippot 2012a), and Ost 1987 (Arif 2017; McKenna 2017), respectively. In each of these studies, the same people who delivered the CBT delivered the relaxation therapy.

- Provision of information about tinnitus and hearing (Abbott 2009; Henry 1996; Nyenhuis 2013a). This included information about tinnitus and its causes, the auditory system, audiological assessment and available treatments. Information about tinnitus was provided by: a clinical psychologist (Henry 1996), an 11-page booklet (Nyenhuis 2013a), and computer/Internet with the support of brief contact from a psychologist to provide new passwords to access new information support regarding their tinnitus coping.

- Internet-based discussion forums (Hesser 2012; Jasper 2014; Weise 2016). The discussion forums were moderated by clinical psychology students (Hesser 2012; Jasper 2014; Weise 2016), licensed CBT therapists (Weise 2016) or licensed psychologists (Hesser 2012). New topics of discussion, such as "representations of tinnitus in the media" (Weise 2016) were presented on a weekly basis in addition to participants being able to initiate topics in each of the respective studies.

- Coping effectiveness training (Martz 2018; Oron (unpublished)) aimed to increase understanding of stress and coping with tinnitus, and how to better learn how to match appropriate coping strategies to situations. The intervention content was delivered in English by psychologists or counsellors (Martz 2018), and in Hebrew (Oron (unpublished)) - no information available about the presenters).

- Masking (Jakes 1992; Zhong 2014). Jakes 1992 used a standard masker device supplied by $A$ and $M$ Hearing Aids Ltd and instructed participants to turn the masker volume up so that they could not hear the tinnitus. Jakes 1992 also included a condition with a placebo masking device that was the same as in the masking condition, but the volume control of the masker was glued into place at the participant's threshold. Zhong 2014 used an MP3 player (no further details provided) as a masking device and participants were instructed to use it one to three times a day, for 15 to 20 minutes each time.

- Virtual reality was delivered in two steps; the first of which included information about tinnitus, treatment and short breathing and relaxation techniques (Malinvaud 2016). The second step where participants entered into a virtual world and were able to control a tinnitus avatar took place under the direction of an ENT physician over eight once-weekly sessions. During a session (one-hour duration) participants were asked to navigate through three environments in which they could choose to displace, mask or unmask sounds as they wished (Malinvaud 2016).

- Self-help. Nyenhuis 2013a included two self-help conditions which varied only in the mode of delivery, i.e. via bibliotherapy or Internet. The content of these conditions was adapted from Tinnitus Coping Training (Kröner-Herwig 1997; Kröner-Herwig 2003). (See also Characteristics of included studies). 
Five studies also included a wait list control condition in addition to an active control group (Henry 1996; Jakes 1992; Malinvaud 2016; Martz 2018; Oron (unpublished).

\section{Outcomes}

\section{Primary outcomes}

Impact of tinnitus on quality of life

Twenty-six of the 28 studies reported changes in the impact of tinnitus on quality of life as measured by scores on a multi-item questionnaire. Fourteen studies used a single multi-item questionnaire (Abbott 2009; Andersson 2002; Andersson 2005; Arif 2017; Beukes 2018a; Davies 1995; Hesser 2012; Jakes 1986; Jakes 1992; Nyenhuis 2013a; Oron (unpublished); Philippot 2012a; Westin 2011; Zhong 2014), nine studies used two multi-item questionnaires (Beukes 2018b; Cima 2012; Jasper 2014; Kaldo 2007; Kreuzer 2012; Malinvaud 2016; McKenna 2017; Schmidt 2018; Weise 2016), two studies used three multi-item questionnaires (Henry 1996; Henry 1998), and one study used four multi-item questionnaires (Robinson 2008).

Of the 14 studies that used one multi-item questionnaire, four used the Tinnitus Handicap Inventory (THI; Newman 1996) (Hesser 2012; Oron (unpublished); Westin 2011; Zhong 2014), four used the Tinnitus Reaction Questionnaire (TRQ; Wilson 1991) (Abbott 2009; Andersson 2002; Andersson 2005; Arif 2017), three used the Tinnitus Effects Questionnaire-Emotional Distress scale (TEQ-ED; Hallam 1988) (Davies 1995; Jakes 1986; Jakes 1992), one used the Tinnitus Functional Index (TFI; Meikle 2012) (Beukes 2018a), one used the Tinnitus Questionnaire (TQ; Hallam 1988; Hallam 2008) (Nyenhuis 2013a), and one used the Tinnitus Psychological Impact Questionnaire (QIPA) (Philippot 2012a). (See explanatory note in Characteristics of included studies, Philippot 2012a regarding the QIPA).

Of the nine studies that used two questionnaires, Beukes 2018b used the TFI and THI; Jasper 2014 and Weise 2016 both used the THI and mini-TQ, Malinvaud 2016 used the THI and THQ; Schmidt 2018 and Kaldo 2007 used the THI and TRQ; Kreuzer 2012 and Cima 2012 used the THI and TQ; and, McKenna 2017 used the TFI and TQ. Henry 1996 and Henry 1998 both used the same three questionnaires (TRQ, THQ, TEQ-ED) and Robinson 2008 used four multi-item questionnaires (THQ, TRQ, THI, TQ).

In this review we measured outcome at treatment end, and at six- and 12-month followup. Three studies reported follow-up data at six months (Jasper 2014; McKenna 2017; Weise 2016), and six studies reported data at 12 months follow-up (Andersson 2002; Henry 1996; Hesser 2012; Kaldo 2007; Robinson 2008; Weise 2016). However, only McKenna 2017 and Henry 1996 reported data from active comparator groups that could be considered. Other studies used wait list control groups and subsequently gave those participants CBT. The time points at which follow-up data were collected in other studies included two weeks (Kreuzer 2012), one month (Davies 1995; Jakes 1986; Martz 2018; McKenna 2017; Zhong 2014), two months (Beukes 2018a; Beukes 2018b; Robinson 2008; Schmidt 2018), three months (Andersson 2005; Jakes 1992; Malinvaud 2016; Philippot 2012a), four months (Cima 2012; Davies 1995; Henry 1996; Jakes 1986; 
Robinson 2008; Zhong 2014), 4.5 months (Westin 2011), eight months (Henry 1996), nine months (Nyenhuis 2013a), and 16 months (Westin 2011). Four studies did not collect follow-up data (Abbott 2009; Arif 2017; Lindberg 1989; Oron (unpublished)).

\section{Serious adverse effects}

Eleven of the 28 studies provided information about the incidence of serious adverse effects. Seven studies reported, or we were informed by the authors via personal communication, that there were no serious adverse effects associated with CBT (Andersson 2002; Andersson 2005; Beukes 2018b; Cima 2012; Kaldo 2007; Malinvaud 2016; Oron (unpublished)). Four studies reported some serious adverse effects (mostly symptom deterioration) in a small number of participants (Beukes 2018a; Nyenhuis 2013a; Weise 2016; Westin 2011). Information about the presence of serious adverse effects was available at the end of treatment (Beukes 2018a; Nyenhuis 2013a; Westin 2011), six-month follow-up (Westin 2011) and 12-month follow-up (Beukes 2018a as reported in Beukes 2018c).

\section{Secondary outcomes \\ Depression}

Depression was measured with multi-item questionnaires in 22 studies. It was measured with the Hospital Anxiety and Depression Scale-Depression subscale (HADS-D; Zigmond 1983) in 11 studies (Andersson 2002; Andersson 2005; Arif 2017; Cima 2012; Hesser 2012; Jasper 2014; Kaldo 2007; Malinvaud 2016; McKenna 2017; Weise 2016; Westin 2011), the Beck Depression Inventory (BDI; Beck 1996) in five studies (Davies 1995; Henry 1996; Henry 1998; Kreuzer 2012; Philippot 2012a), the Patient Health Questionnaire-9 (PHQ-9; Kroenke 2001) in three studies (Beukes 2018a; Beukes 2018b; Nyenhuis 2013a), the Depression Anxiety and Stress Scale-Depression subscale (DASS-D; Lovibond 1995) in one study (Abbott 2009), and the Hamilton Rating Scale for Depression (HRSD; Hamilton 1960) in one study (Jakes 1986). One study used both the HRSD and the BDI to measure depression (Robinson 2008).

\section{Anxiety}

Anxiety was measured with multi-item questionnaires in 16 studies. It was measured with the Hospital Anxiety and Depression Scale-Anxiety subscale (HADS-A; Zigmond 1983) in nine studies (Arif 2017; Cima 2012; Hesser 2012; Jasper 2014; Kaldo 2007; Malinvaud 2016; McKenna 2017; Weise 2016; Westin 2011); the Generalised Anxiety Disorder-7 scale (GAD-7; Spitzer 2006) in two studies (Beukes 2018a; Beukes 2018b); the State Trait Anxiety Inventory (STAl; Spielberger 1983) in two studies (Davies 1995; Philippot 2012a); and the Depression Anxiety and Stress Scale-Anxiety subscale (DASSA; Lovibond 1995) in one study (Abbott 2009). Two studies used both the HADS-A and Anxiety Sensitivity Index (ASI; Reiss 1986) to assess anxiety (Andersson 2002; Andersson 2005).

\section{Quality of life}

Seven studies assessed change in quality of life. Quality of life was assessed with multiitem questionnaires that included the Satisfaction with Life Survey (SWLS; Diener 1985) in two studies (Beukes 2018a; Beukes 2018b); the Quality of Life Inventory (QOLI; Frisch 
1992) in two studies (Hesser 2012; Westin 2011); the Health Utilities Index (HUI; Furlong 2001) in one study (Cima 2012); the Quality of Well-being Scale (Kaplan 1996) in one study (Robinson 2008); and the WHOQoL (Skevington 2004) in one study (Abbott 2009).

\section{Negative biased interpretations of tinnitus}

Biased interpretations of tinnitus were measured with multi-item questionnaires in 10 studies. The Tinnitus Acceptance Questionnaire (TAQ; Weise 2013) was used in five studies (Hesser 2012; Jasper 2014; McKenna 2017; Weise 2016; Westin 2011); the Tinnitus Effects Questionnaire-Irrational Beliefs subscale (TEQ-IB; Hallam 1988) in four studies (Davies 1995; Jakes 1992; Henry 1996; Henry 1998); and the Tinnitus Cognitions Questionnaire (TCQ; Wilson 1998) in two studies (Henry 1996; Henry 1998). Cima 2012 used the Tinnitus Catastrophizing Scale (TCS; Cima 2011b) and Fear of Tinnitus Questionnaire (FTQ; Cima 2011b), and McKenna 2017 used the TAQ (Weise 2013), TCS (Cima 2011b), and Tinnitus-Fear and Avoidance Scale (T-FAS; Kleinstauber 2013).

\section{Other adverse effects}

Only one study reported some 'other' adverse effects (Weise 2016). These adverse effects occurred at 12 months follow-up and referred to some "slight deterioration in sleep quality". (Note: there were no long-term comparator data available as the online discussion comparator group had received CBT by that time).

\section{Non-relevant outcomes}

Two studies did not use any outcomes that were relevant to this review (Lindberg 1989; Martz 2018). Lindberg 1989 reported results from visual analogue scales that were used on a daily basis for one week to measure subjective loudness, discomfort from tinnitus and ability to control the discomfort from tinnitus. Martz 2018 reported results from the Brief COPE scale (Carver 1997), a 28-item version of the COPE inventory (Carver 1989) measuring what techniques, strategies or supports people use to manage challenging situations or experiences. Martz 2018 specifically reported results for three subscales: "engagement coping", which includes, for example, positive re-framing, self-distraction and use of humour, "disengagement coping", which includes, denial, behavioural disengagement and self-blame, and "social support coping", which includes instrumental support, emotional support, venting and religion.

\section{Excluded studies}

From the full-text screening we excluded 15 studies. The main reasons for excluding studies were: non-random allocation of participants (six studies); wrong intervention (six studies); secondary analyses of data (two studies); and no appropriate comparator included (one study). A list of studies and the specific reason for exclusion is in the Characteristics of excluded studies table.

\section{Ongoing studies}

We identified five ongoing studies in our search results (NCT03022084; NCT03386123; NCT04004260; NTR6415; SLCTR/2018/005), which are reported in the Characteristics of ongoing studies table. 


\section{Risk of bias in included studies}

A graph showing a summary of the 'Risk of bias' assessments is shown in Figure 2 and the review authors' judgements for each included study are shown in Figure 3.

Figure 2. Risk of Bias Graph: Review Authors' Judgements about Each Risk of Bias Item Presented as Percentages across All Included Studies

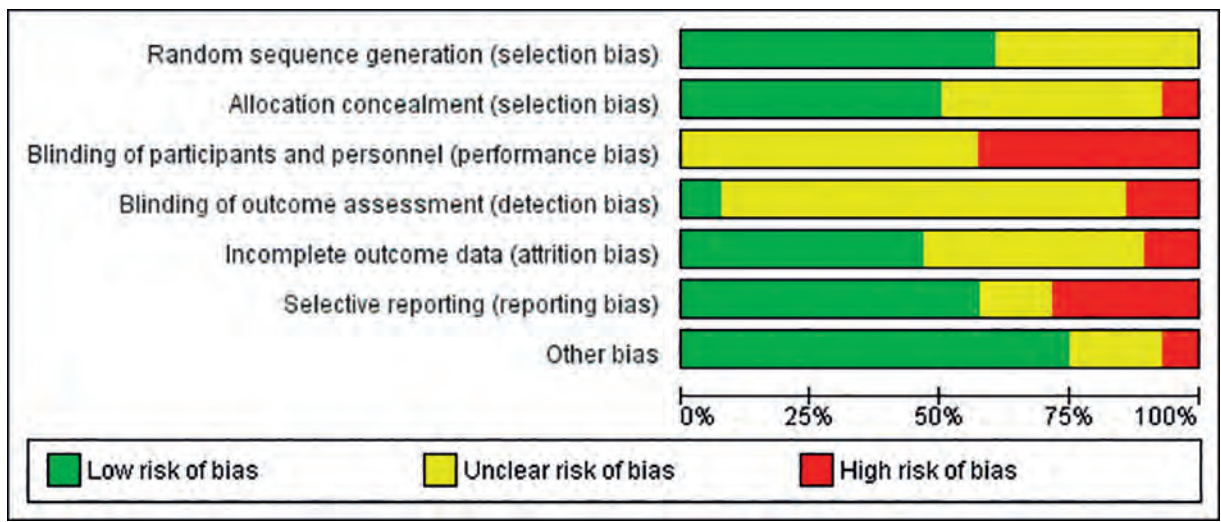

Note: 'Risk of bias' graph: review authors' judgements about each risk of bias item presented as percentages across all included studies.

\section{Allocation (selection bias)}

Random sequence generation

We rated 17 included studies as having low risk of bias because they clearly described or information was received from corresponding authors to confirm an adequate random sequence generation process had been used (Abbott 2009; Beukes 2018a; Beukes 2018b; Cima 2012; Hesser 2012; Jasper 2014; Kaldo 2007; Kreuzer 2012; Malinvaud 2016; Martz 2018; McKenna 2017; Nyenhuis 2013a; Oron (unpublished); Robinson 2008; Schmidt 2018; Weise 2016; Westin 2011). Methods of random sequence generation included Internet-based randomisation systems (e.g. www.random.org, www. randomization.com), coin tossing, computer-generated random number sequences and block randomisation, and drawing numbers from a hat. We rated the remaining 11 studies as having an 'unclear' risk of bias as they did not describe their random sequence generation methods (Andersson 2002; Andersson 2005; Arif 2017; Davies 1995; Henry 1996; Henry 1998; Jakes 1986; Jakes 1992; Lindberg 1989; Philippot 2012a; Zhong 2014). 
Chapter 3

Figure 3. Risk of Bias Summary: Review Authors' Judgments of Risk of Bias Item for Each Included Study

\begin{tabular}{|c|c|c|c|c|c|c|c|}
\hline & 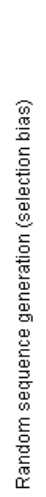 & 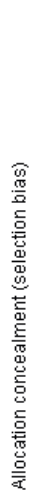 & 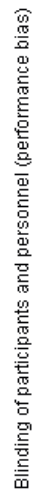 & 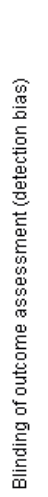 & 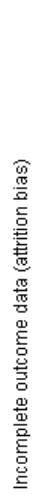 & 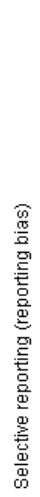 & $\begin{array}{l}\frac{0}{0} \\
\frac{0}{5} \\
\stackrel{5}{2} \\
\stackrel{5}{5}\end{array}$ \\
\hline Abbott 2009 & 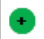 & $?$ & $\odot$ & $?$ & - & $\odot$ & $\odot$ \\
\hline Andersson 2002 & $?$ & + & 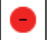 & $?$ & $?$ & $\odot$ & + \\
\hline Andersson 2005 & $?$ & $?$ & $?$ & $?$ & $\oplus$ & $\oplus$ & + \\
\hline Arif 2017 & $?$ & + & $\odot$ & $?$ & $?$ & $\odot$ & + \\
\hline Beukes 2018a & + & - & $\odot$ & $\odot$ & + & $\oplus$ & $\oplus$ \\
\hline Beukes 2018b & + & $\odot$ & $\Theta$ & + & + & 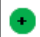 & $\oplus$ \\
\hline Cima 2012 & $\odot$ & $\odot$ & ? & $?$ & $\odot$ & 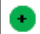 & + \\
\hline Davies 1995 & $?$ & $?$ & $?$ & ? & $\Theta$ & + & $\odot$ \\
\hline Henry 1996 & $?$ & $?$ & $?$ & $?$ & $\oplus$ & 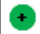 & $\oplus$ \\
\hline Henry 1998 & ? & $?$ & ? & ? & ? & + & + \\
\hline Hesser 2012 & + & + & - & $?$ & + & + & + \\
\hline Jakes 1986 & $?$ & ? & ? & $?$ & ? & - & + \\
\hline Jakes 1992 & ? & $?$ & $?$ & ? & ? & $\Theta$ & + \\
\hline Jasper 2014 & + & + & ? & + & + & ? & $?$ \\
\hline Kaldo 2007 & + & $?$ & $\Theta$ & ? & $\odot$ & + & + \\
\hline Kreuzer 2012 & $\odot$ & $?$ & 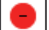 & $\odot$ & ? & + & ? \\
\hline Lindberg 1989 & $?$ & + & $?$ & ? & + & $?$ & ? \\
\hline Malinvaud 2016 & $\odot$ & $\odot$ & $\odot$ & - & $\odot$ & $\odot$ & ? \\
\hline Martz 2018 & + & + & $\Theta$ & $?$ & ? & $\Theta$ & - \\
\hline McKenna 2017 & + & + & ? & $?$ & ? & + & + \\
\hline Nyenhuis 2013a & $\odot$ & $\odot$ & ? & $?$ & ? & + & $\odot$ \\
\hline Oron (unpublished) & $\odot$ & $\odot$ & $\odot$ & $?$ & $?$ & ? & ? \\
\hline Philippot 2012a & ? & $?$ & $?$ & ? & $\odot$ & $\oplus$ & $\odot$ \\
\hline Robinson 2008 & + & + & - & ? & ? & - & + \\
\hline Schmidt 2018 & + & $?$ & $?$ & $\Theta$ & + & $\Theta$ & - \\
\hline Weise 2016 & $\odot$ & $\odot$ & $?$ & $?$ & $\odot$ & $\Theta$ & + \\
\hline Westin 2011 & + & $\Theta$ & ? & $?$ & $?$ & + & + \\
\hline Zhong 2014 & $?$ & $?$ & $?$ & $?$ & $\odot$ & $?$ & + \\
\hline
\end{tabular}




\section{Allocation concealment}

We rated half the included studies as having a low risk of bias for allocation concealment as it was performed by staff independent of the research team, or was conducted centrally (Andersson 2002; Arif 2017; Beukes 2018b; Cima 2012; Hesser 2012; Jasper 2014; Lindberg 1989; Malinvaud 2016; Martz 2018; McKenna 2017; Nyenhuis 2013a; Oron (unpublished); Robinson 2008; Weise 2016). We rated two studies as being at high risk of bias because there was no allocation concealment (Beukes 2018a; Westin 2011). In the remaining 12 studies, allocation concealment was not described and so we rated those studies as having unclear risk of bias (Abbott 2009; Andersson 2005; Davies 1995; Henry 1996; Henry 1998; Jakes 1986; Jakes 1992; Kaldo 2007; Kreuzer 2012; Philippot 2012a; Schmidt 2018; Zhong 2014).

\section{Blinding (performance bias and detection bias)}

\section{Blinding of participants and personnel}

We judged 12 studies to be at high risk of bias (Abbott 2009; Andersson 2002; Arif 2017; Beukes 2018a; Beukes 2018b; Hesser 2012; Kaldo 2007; Kreuzer 2012; Malinvaud 2016; Martz 2018; Oron (unpublished); Robinson 2008), and the remaining 16 to be at unclear risk of bias in relation to blinding of participants and personnel (Andersson 2005; Cima 2012; Davies 1995; Henry 1996; Henry 1998; Jakes 1986; Jakes 1992; Jasper 2014; Lindberg 1989; McKenna 2017; Nyenhuis 2013a; Philippot 2012a; Schmidt 2018; Weise 2016; Westin 2011; Zhong 2014). Although CBT itself is not possible to 'mask', we judged there to be a high risk where there was a clear difference between the intervention and comparison group. Specifically, we judged there to be a high risk when there was a wait list control (e.g. Andersson 2002), when an information only control was used (e.g. Abbott 2009), and when participants were explicitly informed of the differences between groups/interventions that they could be allocated to (e.g. Arif 2017). In other scenarios, for example, where participants might not know the difference between the intervention and control, and/or if the content of the alternative treatment was masked, we rated the risk of bias as unclear (e.g. Cima 2012).

\section{Blinding of outcome assessment}

We rated two studies as having a low risk of bias (Beukes 2018b; Jasper 2014) and four studies as having a high risk of bias with regard to the likely impact that unblinded outcome assessment had on the study results (Beukes 2018a; Kreuzer 2012; Malinvaud 2016; Schmidt 2018). We rated Beukes 2018 b as low risk of bias as the data analysts were blinded to group allocation. Jasper 2014 specifically investigated the perceived expectations and credibility of the intervention (iCBT) and control (online discussion forum), and concluded that there was no effect of these on the results. The four studies rated as high risk of bias due to lack of blinding all reported that outcome assessors were not blinded. The remaining 22 studies did not report sufficient detail to judge whether outcome assessors were blinded and so we rated them as having unclear risk of detection bias. 


\section{Incomplete outcome data (attrition bias)}

We rated three studies as having a high risk (Abbott 2009; Davies 1995; Malinvaud 2016), 12 an unclear risk (Andersson 2002; Arif 2017; Henry 1998; Jakes 1986; Jakes 1992; Kreuzer 2012; Martz 2018; McKenna 2017; Nyenhuis 2013a; Oron (unpublished); Robinson 2008; Westin 2011), and 13 a low risk of attrition bias (Andersson 2005; Beukes 2018a; Beukes 2018b; Cima 2012; Henry 1996; Hesser 2012; Jasper 2014; Kaldo 2007; Lindberg 1989; Philippot 2012a; Schmidt 2018; Weise 2016; Zhong 2014).

Abbott 2009 reported a high level of attrition, most of which was unexplained, and replaced missing data with the last (observed) outcome carried forward. Davies 1995 reported differing levels of attrition across groups and concluded from this that the "differential attrition to some extent invalidates the group comparison". Malinvaud 2016 reported that different reasons were given by participants for dropout depending on the group they were allocated to, and missing data appear to be related to treatment outcome. Hence we also rated this study as having a high risk of attrition bias.

\section{Selective reporting (reporting bias)}

We judged eight studies to be at high risk of reporting bias. These included studies with discrepancies between what was reported in the protocol or methods and what was published in the results (Abbott 2009; Jakes 1992; Malinvaud 2016; Martz 2018; Robinson 2008; Weise 2016). For example, Schmidt 2018 specified a primary outcome in the study protocol but did not report it as such in the publication, and additional outcomes not identified in protocol were reported in the publication. Three studies did not report enough information to judge the risk of reporting bias (Jasper 2014; Lindberg 1989; Zhong 2014), and one is currently being prepared for publication and hence we judged it as at unclear risk (Oron (unpublished)). We rated all other studies as low risk, having either fully reported what was specified in their respective methods sections, and/ or reported everything as specified in a published protocol or trial registration (Arif 2017 Beukes 2018b; Cima 2012; Davies 1995; Henry 1996; Henry 1998; Hesser 2012; Kaldo 2007; Kreuzer 2012; McKenna 2017; Nyenhuis 2013a; Philippot 2012a; Westin 2011).

\section{Other potential sources of bias}

We judged two studies to be at risk of other biases. Martz 2018 did not explain why the initial anticipated sample size estimate was 80 but only 40 participants were included in the final publication, or why ACT was added as an intervention arm one year after initial trial registration. In Schmidt 2018 there were additional discrepancies between the protocol and publication, e.g. the protocol included a "no-intervention/standard care condition" but this was not reported in the publication of the study.

Four studies received an unclear rating for various reasons. In Jasper 2014 the inclusion criteria differed between the protocol (mini TQ score over 12) and study publications (mini TQ score over 8). In Kreuzer 2012 the long waiting time could lead to increased spontaneous improvements in the control group. Lindberg 1989 reported no scientific input from funders, but no protocol was available to inform our judgement. In Malinvaud 2016 there were some deviations from the protocol with an unknown effect on the 
outcomes of the study. Oron (unpublished) is not yet published. We judged all other studies as having a low risk of other biases.

\section{Effects of interventions}

Comparison 1: Cognitive behavioural therapy (CBT) versus no intervention/wait list control

\section{Primary outcomes}

1.1 Impact of tinnitus on quality of life

\subsubsection{End of treatment}

Ten studies reported data on the impact of tinnitus on quality of life at the end of treatment using validated multi-item questionnaires (Andersson 2002; Andersson 2005; Beukes 2018a; Henry 1996; Jakes 1992; Kaldo 2007; Kreuzer 2012; Malinvaud 2016; Oron (unpublished); Westin 2011). (Note: Post-treatment data from Malinvaud 2016 were not available and so, in accordance with a conservative approach, we entered baseline data showing no difference between the groups. This note applies to all analyses where Malinvaud 2016 is included). Overall, there was a clear difference in favour of CBT indicating that it may lead to a reduction in the impact of tinnitus on quality of life (standardised mean difference (SMD) -0.56, 95\% confidence interval (Cl) -0.83 to -0.30; 10 studies; 537 participants; Analysis 1.1). Re-expressed as a score on the Tinnitus Handicap Inventory (THI; range 0 to 100) this is equivalent to a score 10.91 points lower in the CBT group, with an estimated minimal clinically important difference (MCID) for this scale being 7 points. A moderate level of heterogeneity was present $\left(\operatorname{Tau}^{2}=0.08\right.$; $\left.\mathrm{Chi}^{2}=17.62, \mathrm{df}=9(\mathrm{P}=0.04) ; \mathrm{I}^{2}=49 \%\right)$. The forest plot illustrating this result is shown in Figure 4 and it is reported in Summary of findings table 1 as a finding of importance. We rated the certainty of the evidence as low.

Subgroup analyses examining the type of therapy compared to wait list control indicated that there no statistically significant differences between the types of therapy, and that heterogeneity might not be important $\left(\mathrm{Chi}^{2}=1.80\right.$, df $=3(\mathrm{P}=0.61), \mathrm{I}^{2}=0 \%$; Analysis 1.7): six studies used CBT (Andersson 2002; Andersson 2005; Beukes 2018a; Jakes 1992; Kaldo 2007; Malinvaud 2016), two used acceptance and commitment therapy (ACT) (Oron (unpublished); Westin 2011), one used cognitive therapy (Henry 1996), and one used mindfulness (Kreuzer 2012). 
Chapter 3

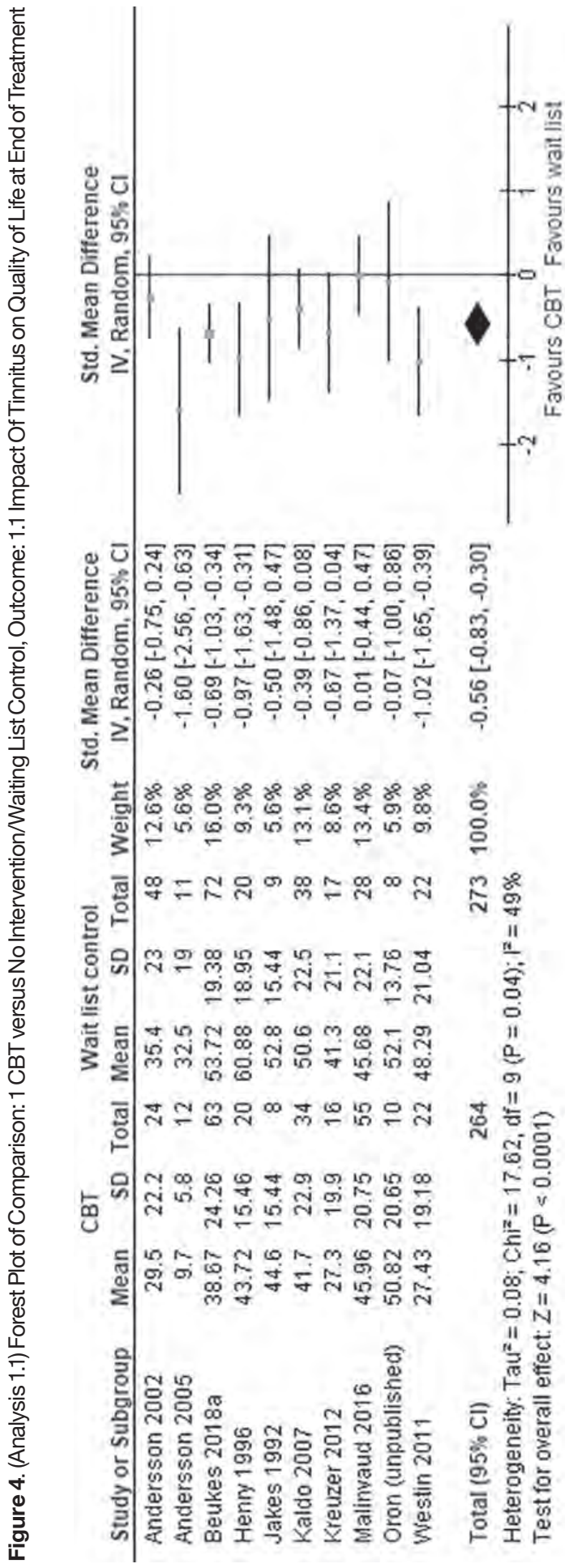


Subgroup analyses examining the mode of delivery (bibliotherapy, face-to-face and Internet-based) indicated that there were no significant differences between the modes of delivery, and that heterogeneity might not be important $\left(\mathrm{Chi}^{2}=0.69, \mathrm{df}=2(\mathrm{P}=0.71)\right.$, $\mathrm{I}^{2}=0 \%$; Analysis 1.9). Seven of the studies in this analysis delivered therapy face-toface (Andersson 2005; Henry 1996; Jakes 1992; Kreuzer 2012; Malinvaud 2016; Oron (unpublished); Westin 2011), two via Internet interventions (Andersson 2002; Beukes 2018a), and one by bibliotherapy (Kaldo 2007).

When the unit of delivery was examined (i.e. individual compared with group), the subgroup analyses detected no significant differences and indicated that heterogeneity might not be a problem $\left(\mathrm{Chi}^{2}=0.01, \mathrm{df}=1(\mathrm{P}=0.94), \mathrm{I}^{2}=0 \%\right.$; Analysis 1.11$)$ : six studies delivered treatment in groups (Andersson 2005; Henry 1996; Jakes 1992; Kreuzer 2012; Malinvaud 2016; Oron (unpublished)), and four delivered it individually (Andersson 2002; Beukes 2018a; Kaldo 2007; Westin 2011).

Subgroup analyses by who delivered CBT (i.e. psychologists, "other clinician", computer, bibliotherapy) indicated no difference, and that heterogeneity might not be a problem $\left(\mathrm{Chi}^{2}=1.65, \mathrm{df}=3(\mathrm{P}=0.65), \mathrm{I}^{2}=0 \%\right.$; Analysis 1.13): four studies reported that psychologists delivered treatment (Henry 1996; Malinvaud 2016; Oron (unpublished); Westin 2011), three employed "other clinicians" (Andersson 2005; Jakes 1992; Kreuzer 2012), two used computers/Internet interventions (Andersson 2002; Beukes 2018a), and one used bibliotherapy (Kaldo 2007).

No data were available for subgroup analysis comparing selection of participants based on inclusion/exclusion criteria relating to severe hearing loss.

\subsubsection{Six months follow-up}

One study collected six-month follow-up data (Henry 1998), but by this time the participants in the wait list control group had received CBT and thus there was no comparison available.

\subsubsection{Twelve months follow-up}

Four studies collected 12-month follow-up data (Andersson 2002; Henry 1996; Kaldo 2007; Robinson 2008), but participants in the wait list groups had all received CBT treatment by that time and thus at the 12-month time point no comparison could be made.

\subsection{Serious adverse effects}

\subsubsection{End of treatment}

Seven studies, rated as moderate certainty, either reported or informed us via personal communication about serious adverse effects. Six informed us that no serious adverse effects occurred in the CBT or wait list condition at the end of treatment (Andersson 2002; Andersson 2005; Beukes 2018a; Kaldo 2007; Malinvaud 2016; Oron (unpublished)) and one study (Westin 2011) reported that one participant had deteriorated according to calculations of the reliable change index (Jacobson 1991) using the THI scores. This deterioration, however, occurred in the period between pre-treatment assessment 
and the assessment following the first session but still appeared in the data at end of treatment. Given the timing of the deterioration, it is unlikely to be related to CBT (or specifically, ACT).

\subsubsection{Six months follow-up}

Westin 2011 reported at six-month follow-up that one participant of the 22 in the ACT condition had, according to reliable change calculations, deteriorated. This deterioration occurred in the period between pre-treatment assessment to the assessment following the first session (hence it was unlikely to be caused by CBT) but was still detectable at six-month follow-up.

\subsubsection{Twelve months follow-up}

At 12-month follow-up Beukes 2018c reported that three participants from the study Beukes 2018a developed "moderate" new symptoms, "moderate" negative wellbeing, and that two participants had thought that treatment was too long.

\section{Secondary outcomes}

\subsection{Depression}

\subsubsection{End of treatment}

Eight studies reported scores on measures of depression at the end of treatment (Andersson 2002; Andersson 2005; Beukes 2018a; Henry 1996; Kaldo 2007; Kreuzer 2012; Malinvaud 2016; Westin 2011). There was a statistically significant difference in favour of CBT (SMD $-0.34,95 \%$ Cl -0.60 to -0.08 ; 8 studies; 502 participants; Analysis 1.3) while moderate heterogeneity might be present $\left(\mathrm{Tau}^{2}=0.06\right.$; $\mathrm{Chi}^{2}=12.85, \mathrm{df}=7$ $\left.(P=0.08) ; I^{2}=46 \%\right)$. This result was reported in Summary of findings table 1 . Overall we rated the certainty of the evidence for this outcome as low.

\subsubsection{Six months follow-up}

No data were available for this outcome at this time point.

\subsubsection{Twelve months follow-up}

No data were available for this outcome at this time point.

\subsection{Anxiety}

\subsubsection{End of treatment}

Six studies reported scores on multi-item questionnaires measuring levels of anxiety at the end of treatment (Andersson 2002; Andersson 2005; Beukes 2018a; Kaldo 2007; Malinvaud 2016; Westin 2011). There was a statistically significant difference in favour of CBT (SMD -0.45, 95\% Cl -0.82 to -0.09; 6 studies; 429 participants; Analysis 1.4). Substantial statistical heterogeneity was present $\left(\mathrm{Tau}^{2}=0.13 ; \mathrm{Chi}^{2}=15.36, \mathrm{df}=5(\mathrm{P}=0.009)\right.$; $\left.\mathrm{I}^{2}=67 \%\right)$. We rated the certainty of the evidence for this outcome as very low meaning that the true effect is likely to be substantially different from the estimate of effect.

\subsubsection{Six months follow-up}

No data were available for this outcome at this time point.

\subsubsection{Twelve months follow-up}


No data were available for this outcome at this time point.

\subsection{Health-related quality of life}

\subsubsection{End of treatment}

Two studies collected health-related quality of life data and the results indicated that there was a significant difference in favour of CBT (SMD $-0.38,95 \% \mathrm{Cl}-0.67$ to -0.08 ; 2 studies; 179 participants; Analysis 1.5). Heterogeneity was not likely to be important $\left(\mathrm{Tau}^{2}=0.00 ; \mathrm{Chi}^{2}=0.26, \mathrm{df}=1(\mathrm{P}=0.61) ; \mathrm{I}^{2}=0 \%\right)$. Beukes 2018a used the Satisfaction with Life Scales and reported that the CBT group's quality of life improved significantly more than the wait list control group. Westin 2011 used the Quality of Life Index and linear mixed model regression to examine group by time effects over the 18-month follow-up period of the study. Although means and standard deviations were reported at the end of the treatment phase for the groups (i.e. ACT, TRT and wait list control) the differences between the groups were not analysed at this time point.

We rated the certainty of the evidence as very low meaning that the true effect is likely to be substantially different from the estimate of effect.

\subsubsection{Six months follow-up}

No data were available for this outcome at this time point.

\subsubsection{Twelve months follow-up}

No data were available for this outcome at this time point.

\subsection{Negatively biased interpretations of tinnitus}

\subsubsection{End of treatment}

Two studies reported multi-item questionnaire measures of negatively biased interpretations of tinnitus (Henry 1996; Westin 2011). The analysis of the combined data revealed no difference between the CBT and wait list control groups. We rated the certainty of the evidence as very low, which suggests that the true effect is likely to be substantially different from the estimate of effect. There was high statistical heterogeneity $\left(I^{2}=73 \%\right)$, which could be explained by the different CBT interventions and units of delivery: Henry 1996 compared group cognitive therapy whereas Westin 2011 compared ACT delivered to individuals. Both studies, however, employed psychologists to provide the face-to-face intervention.

\subsubsection{Six months follow-up}

No data were available for this outcome at this time point.

\subsubsection{Twelve months follow-up}

No data were available for this outcome at this time point.

\subsection{Other adverse effects}

\subsubsection{End of treatment}


Seven studies, rated as moderate certainty, either reported or informed us via personal communication that no other adverse effects occurred in the CBT or wait list conditions at the end of treatment (Andersson 2002; Andersson 2005; Beukes 2018a; Kaldo 2007; Malinvaud 2016; Oron (unpublished); Westin 2011).

\subsubsection{Six months follow-up}

No data were available for this outcome at this time point.

\subsubsection{Twelve months follow-up}

No data were available for this outcome at this time point.

\section{Comparison 2: CBT versus usual audiological care}

Three studies compared CBT to audiological care (Beukes 2018b; Cima 2012; Schmidt 2018). No follow-up data were available at 6 or 12 months following the interventions. Two studies reported follow-up data at two months (Beukes 2018b; Schmidt 2018) and one at four months (Cima 2012).

We did not conduct subgroup analyses for this comparison as only three studies were included.

\section{Primary outcomes}

\subsection{Impact of tinnitus on quality of life}

\subsubsection{End of treatment}

Three studies each reported results using the THI and thus we pooled and analysed the data using mean differences (MD). The results indicated a statistically significant difference in favour of CBT compared to usual audiological care (MD $-5.65,95 \% \mathrm{CI}$ -9.79 to -1.50 ; 3 studies; 430 participants; MCID $=7$ points; Analysis 2.1). Statistical heterogeneity was unlikely to be important $\left(\mathrm{Tau}^{2}=0.00\right.$; $\mathrm{Chi}^{2}=0.08, \mathrm{df}=2(\mathrm{P}=0.96)$; $\mathrm{I}^{2}=0 \%$ ). The forest plot is shown in Figure 5 . We rated the certainty of the evidence as moderate reflecting that we are moderately confident that the true effect is likely to be close to the effect estimate but that there is a possibility that it could be substantially different (Summary of findings table 2). 


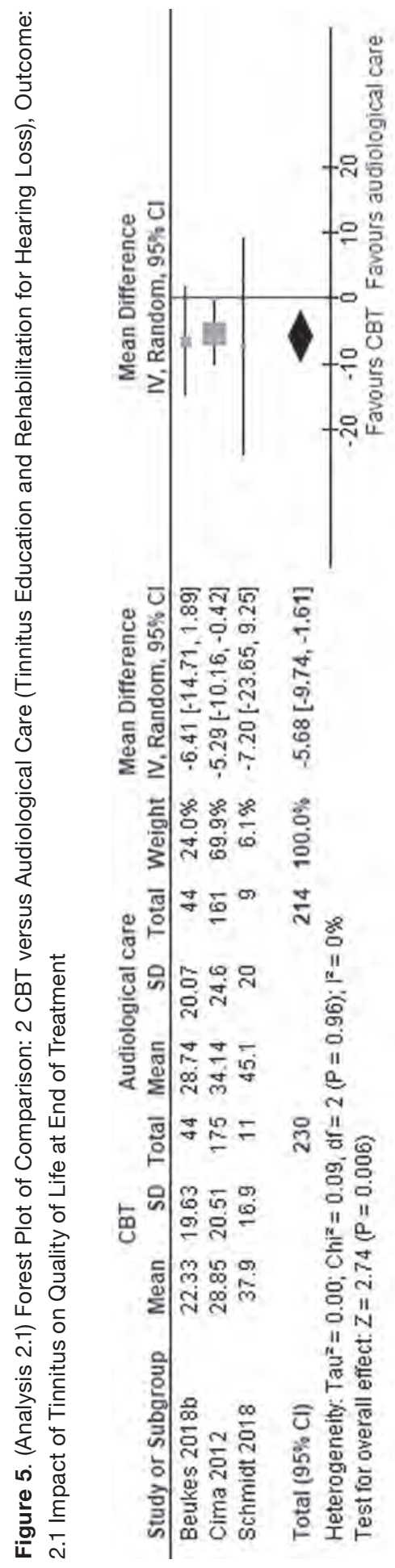


Chapter 3

\subsubsection{Six months follow-up}

No data were available for this outcome at this time point.

\subsubsection{Twelve months follow-up}

No data were available for this outcome at this time point.

\subsection{Serious adverse effects}

\subsubsection{End of treatment}

Two studies reported that no serious adverse effects occurred as a result of the intervention (Beukes 2018b; Cima 2012), and one study did not report information related to this outcome (Schmidt 2018). We rated the certainty of the evidence as moderate for this outcome (Summary of findings table 2).

\subsubsection{Six months follow-up}

No data were available for this outcome at this time point.

\subsubsection{Twelve months follow-up}

No data were available for this outcome at this time point.

\section{Secondary outcomes}

\subsection{Depression}

\subsubsection{End of treatment}

Beukes 2018b used the PHQ-9 and Cima 2012 used the HADS-D to measure depression on multi-item questionnaires. The results indicated that there was no difference between CBT and audiological care (Analysis 2.3). We rated the certainty of the evidence as low suggesting that the true effect is likely to be different from the estimate of the effect (Summary of findings table 2).

\subsubsection{Six months follow-up}

No data were available for this outcome at this time point.

\subsubsection{Twelve months follow-up}

No data were available for this outcome at this time point.

\subsection{Anxiety}

\subsubsection{End of treatment}

Beukes 2018b used the Generalised Anxiety Disorder-7 (GAD-7) scale and Cima 2012 the Hospital Anxiety and Depression Scale - Anxiety subscale (HADS-A) to measure anxiety on multi-item questionnaires. The results indicated that there was no difference between CBT and audiological care (Analysis 2.4). We rated the certainty of the evidence as low suggesting that the true effect is likely to be different from the estimate of the effect (Summary of findings table 2).

\subsubsection{Six months follow-up}

No data were available for this outcome at this time point.

\subsubsection{Twelve months follow-up}


No data were available for this outcome at this time point.

\subsection{Health-related quality of life}

\subsubsection{End of treatment}

Beukes 2018b used the Satisfaction with Life Scale (SLWS) and Cima 2012 used the Health Utilities Index (HUI) to measure quality of life on multi-item questionnaires. The results indicated that there was no difference between CBT and audiological care (Analysis 2.5). We rated the certainty of the evidence as low, suggesting that the true effect is likely to be different from the estimate of the effect (Summary of findings table 2).

\subsubsection{Six months follow-up}

No data were available for this outcome at this time point.

\subsubsection{Twelve months follow-up}

No data were available for this outcome at this time point.

\subsection{Negatively biased interpretations of tinnitus}

\subsubsection{End of treatment}

Cima 2012 used the Tinnitus Catastrophizing Scale to measure negatively biased interpretations of tinnitus. At the end of treatment there was a statistically significant difference in favour of CBT based on an intention-to-treat analysis (group difference-4.683, $95 \% \mathrm{Cl}-6.938$ to $-2.428, \mathrm{P}<0.0001$ ), which equated to a moderate effect size (Cohen's $\mathrm{d}=0.60$ ). We rated the certainty of the evidence as low, suggesting that the true effect is likely to be different from the estimate of the effect (Summary of findings table 2).

\subsubsection{Six months follow-up}

No data were available for this outcome at this time point.

\subsubsection{Twelve months follow-up}

No data were available for this outcome at this time point.

\subsection{Other adverse effects}

No other adverse effects were reported at the end of treatment or at 6 and 12 months respectively in any of the three studies that compared CBT to usual audiological care. We rated the certainty of the evidence as moderate for this outcome (Summary of findings table 2).

\section{Comparison 3: CBT versus tinnitus retraining therapy (TRT)}

One study compared a CBT intervention (ACT) with TRT and a wait list control condition (Westin 2011). For this comparison the wait list control group was included in the analyses reported in Comparison 1.

We did not conduct subgroup analyses for this comparison as only one study was included.

We assessed the certainty of the evidence for all the outcomes in this comparison as low (Summary of findings table 3). 
Chapter 3

\section{Primary outcomes}

\subsection{Impact of tinnitus on quality of life}

Westin 2011 conducted linear mixed model analyses, which revealed a significant linear (time by group) interaction effect on the main outcome measure (THI) at all time points in the study (post-treatment, 6-month and 18-month follow-up; $F(1,114)=8.49 ; P=0.04$ ).

\subsubsection{End of treatment}

At end of treatment, there was a statistically significant difference in favour of ACT compared with TRT (MD -15.79, 95\% Cl -27.91 to -3.67; 1 study; 42 participants; Analysis 3.1).

\subsubsection{Six months follow-up}

At six months follow-up, there was a statistically significant difference in favour of ACT compared with TRT (MD $-13.10,95 \%$ Cl -26.08 to - 0.12; 1 study; 42 participants Analysis 3.2).

\subsubsection{Twelve months follow-up}

No data were available for this outcome at this time point.

\subsection{Serious adverse effects}

\subsubsection{End of treatment}

Three participants deteriorated over the course of the study: one participant was in the ACT group $(n=22)$, and two participants were in the TRT group $(n=20)$. (See section 1.2.1 for information about the participant in the ACT group who deteriorated according to reliable change index calculations (Jacobson 1991; Westin 2011)).

\subsubsection{Six months follow-up}

The two participants in the TRT condition who deteriorated did so between 10 weeks and six months, although they also continued use of the wearable noise generators. (See section 1.2.2 for information about the participant in the ACT group who deteriorated (Westin 2011)).

\subsubsection{Twelve months follow-up}

No data were available for this outcome at this time point.

\section{Secondary outcomes}

\subsection{Depression}

\subsubsection{End of treatment}

At the end of treatment there were no time, group or interaction effects on depression scores as measured by the HADS-D (Westin 2011).

\subsubsection{Six months follow-up}

At six months follow-up there were no time, group or interaction effects on depression scores as measured by the HADS-D (Westin 2011).

\subsubsection{Twelve months follow-up}

No data were available for this outcome at this time point. 


\subsection{Anxiety}

\subsubsection{End of treatment}

At the end of treatment there were no time, group or interaction effects on anxiety scores as measured by the HADS-A (Westin 2011).

\subsubsection{Six months follow-up}

At six months after treatment there were no time, group or interaction effects on anxiety scores as measured by the HADS-A (Westin 2011).

\subsubsection{Twelve months follow-up}

No data were available for this outcome at this time point.

\subsection{Health-related quality of life}

\subsubsection{End of treatment}

At the end of treatment there were no time, group or interaction effects on health-related quality of life scores as measured by the Quality of Life Inventory (QOLI) (Westin 2011).

\subsubsection{Six months follow-up}

At six months after treatment there were no time, group or interaction effects on healthrelated quality of life scores as measured by the QOLI (Westin 2011)

\subsubsection{Twelve months follow-up}

No data were available for this outcome at this time point.

\subsection{Negatively biased interpretations of tinnitus}

Westin 2011 used the Tinnitus Acceptance Questionnaire (TAQ) as a process measure rather than an outcome measure. Before treatment no differences were found between the three groups (ACT, TRT, wait list) concerning tinnitus acceptance $F(2,61)=1.21$; $\mathrm{P}=0.31$ (higher scores indicate better levels of tinnitus acceptance).

\subsubsection{End of treatment}

At the end of treatment there was a clear difference in favour of CBT compared with TRT (MD -9.78, 95\% Cl -16.40 to -3.16; 1 study; 42 participants; Analysis 3.4).

\subsubsection{Six months follow-up}

At 6 months follow-up, there was a difference in favour of CBT compared with TRT (MD $-8.28,95 \% \mathrm{Cl}-15.34$ to -1.22 ; 1 study; 42 participants; Analysis 3.5$)$.

\subsubsection{Twelve months follow-up}

No data were available for this outcome at this time point.

\subsection{Other adverse effects}

3.7.1 End of treatment

No data were available for this outcome at this time point. 
Chapter 3

\subsubsection{Six months follow-up}

No data were available for this outcome at this time point.

\subsubsection{Twelve months follow-up}

No data were available for this outcome at this time point.

\section{Comparison 4: CBT versus other active control}

Sixteen studies compared CBT to an active experimental control group not otherwise included in the previous comparisons (Abbott 2009; Arif 2017; Davies 1995; Henry 1996; Hesser 2012; Jakes 1986; Jakes 1992; Jasper 2014; Malinvaud 2016; Martz 2018; McKenna 2017; Nyenhuis 2013a; Oron (unpublished); Philippot 2012a; Weise 2016; Zhong 2014). Four of these studies did not provide any data that could be included in meta-analyses (Jakes 1986; Martz 2018; Philippot 2012a; Zhong 2014). For a description of the types of active comparison conditions used see Description of studies.

Abbott 2009 used a cluster-randomised controlled trial design but did not report the intracluster correlation coefficients and did not take into consideration the clustered nature of the data in their analyses. In consultation with a statistician, we obtained an estimate of an appropriate intracluster correlation coefficient from Meijerink 2017 and used this to adjust the sample size for the study.

\section{Primary outcomes}

\subsection{Impact of tinnitus on quality of life}

\subsubsection{End of treatment}

Twelve studies provided data on the impact of tinnitus on quality of life using multiitem questionnaires. Analysis indicated that the results were clearly in favour of CBT regardless of what the active control condition was (SMD $-0.30,95 \% \mathrm{Cl}-0.55$ to -0.05; 12 studies; 966 participants; Analysis 4.1). The forest plot is shown in Figure 6. Overall we rated the certainty of the evidence as low, which indicates that the true effect might be substantially different from the effect estimate (Summary of findings table 4). Heterogeneity was moderate to substantial $\left(\mathrm{Tau}^{2}=0.12 ; \mathrm{Chi}^{2}=33.27, \mathrm{df}=11\right.$ $\left.(P=0.0005), I^{2}=67 \%\right)$.

Subgroup analysis examining the type of therapy indicated that there were no significant differences between the types of therapy, and that heterogeneity was not a problem $\left(\mathrm{Chi}^{2}=0.29, \mathrm{df}=3 ; \mathrm{P}=0.96 ; \mathrm{I}^{2}=0 \%\right.$; Analysis 4.14).

Subgroup analysis comparing face-to-face delivery to Internet-based delivery of CBT indicated that there was no significant difference between the respective modes of delivery $\left(\mathrm{Chi}^{2}=1.54, \mathrm{df}=1(\mathrm{P}=0.21), \mathrm{I}^{2}=35 \%\right.$; Analysis 4.16$)$

Subgroup analysis examining whether there was a difference between CBT delivered in groups compared to individually found no difference $\left(\mathrm{Chi}^{2}=1.328\right.$, $\mathrm{df}=1 ; \mathrm{P}=0.25$; $\mathrm{I}^{2}=24 \%$; Analysis 4.17). 
There were no significant group differences regarding who delivers the CBT (psychologists, Internet-based or "therapists") $\left(\mathrm{Chi}^{2}=0.15\right.$, df $=2 ; \mathrm{P}=0.93 ; \mathrm{I}^{2}=0 \%$; Analysis 4.18).

No data were available for subgroup analysis comparing selection of participants based on inclusion/exclusion criteria relating to severe hearing loss. 
Chapter 3

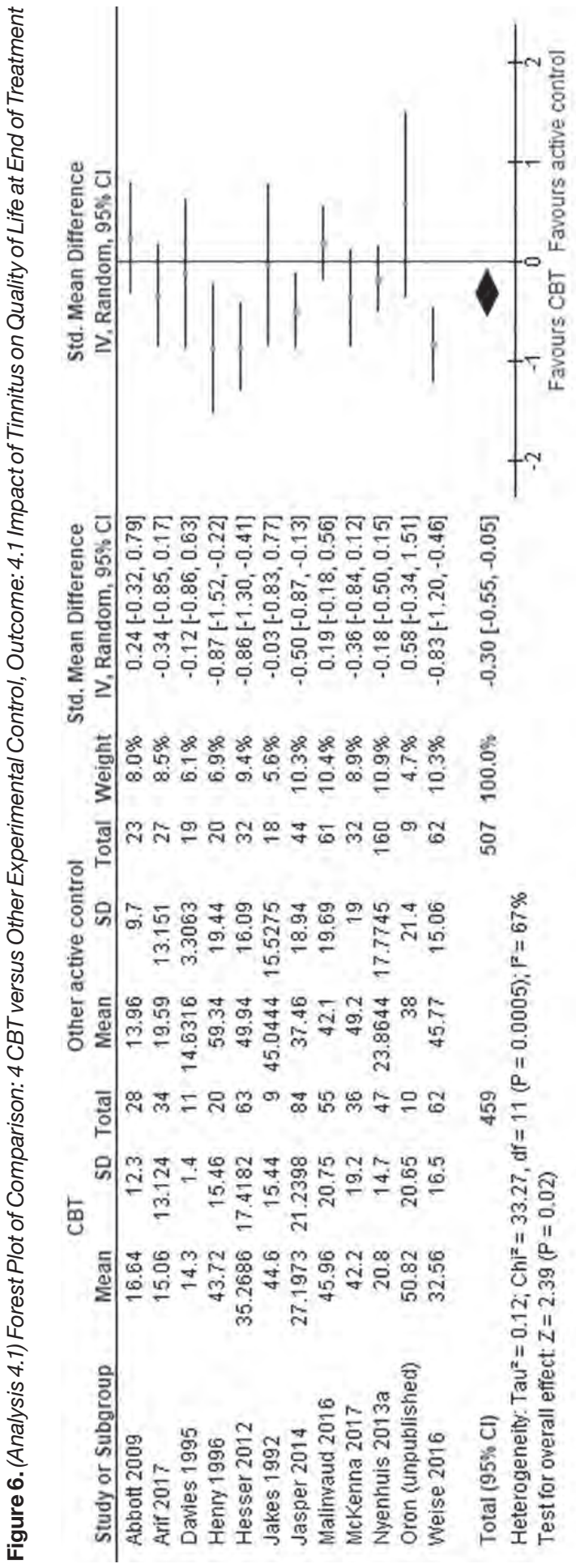




\subsubsection{Six months follow-up}

McKenna 2017 reported that at six months after treatment there was a statistically significant difference in favour of CBT compared to relaxation therapy in the impact of tinnitus on quality of life, as measured by the Tinnitus Questionnaire (TQ). Specifically, the adjusted mean score in Mindfulness Based Cognitive Therapy (MBCT) (mean $=23$, $\mathrm{SD}-18.1)$ was 7.2 points lower $(95 \% \mathrm{Cl} 2.1-12.3, \mathrm{P}=0.006)$ than in relaxation therapy (mean $=35.6, \mathrm{SD}=16.8)$, with a standardised effect size of $0.56(95 \% \mathrm{Cl} 0.16$ to 0.96$)$.

\subsubsection{Twelve months follow-up}

One study provided 12-month follow-up data comparing cognitive therapy combined with education to education only, on the impact of tinnitus on quality of life, as measured by the THQ (Henry 1996). Results from repeated measures ANOVAs indicated that there was no significant difference between the conditions.

\subsection{Serious adverse effects}

\subsubsection{End of treatment}

Nyenhuis 2013a reported that three participants deteriorated using the scores from the TQ in reliable change index calculations (Jacobson 1991); one participant was from the group CBT intervention and two from the information only control. We assessed the certainty of the evidence as low, meaning that the true effect could be substantially different from the effect estimate (Summary of findings table 4).

\subsubsection{Six months follow-up}

No data were available for this outcome at this time point.

\subsubsection{Twelve months follow-up}

Weise 2016 reported that three participants showed "reliable deterioration" using the reliable change index for measures of the impact of tinnitus on quality of life (THI, $n=2$; Mini-TQ, $n=1$ ). (Note: by this time point there were no longer comparator participants since they had been offered and received CBT).

\section{Secondary outcomes}

\subsection{Depression}

\subsubsection{End of treatment}

Eleven studies supplied data for this analysis (Abbott 2009; Arif 2017; Davies 1995; Henry 1996; Hesser 2012; Jasper 2014; Malinvaud 2016; McKenna 2017; Nyenhuis 2013a; Philippot 2012a; Weise 2016). The results indicated a small difference in favour of CBT over other active interventions (SMD -0.17, 95\% Cl -0.33 to -0.01; 11 studies; 943 participants; Analysis 4.5), and heterogeneity was unlikely to be important $\left(\mathrm{Chi}^{2}=13.00, \mathrm{df}=10 ; \mathrm{P}=0.22\right.$; $\mathrm{I}^{2}=23 \%$ ). We assessed the certainty of the evidence as low, meaning that the true effect could be substantially different from the effect estimate (Summary of findings table 4).

\subsubsection{Six months follow-up}

At six months follow-up, McKenna 2017 reported that there were no differences between the MBCT group and active control (relaxation therapy) in HADS-D scores after pre- 
treatment scores had been taken into consideration (MD $-1.90,95 \% \mathrm{Cl}-3.87$ to $0.07 ; 1$ study; 62 participants; Analysis 4.6).

\subsubsection{Twelve months follow-up}

Henry 1996 compared cognitive therapy combined with education to an education only control condition, and reported that there was no significant difference between the groups (MD -2.00, 95\% Cl -7.88 to 3.88; 1 study; 33 participants; Analysis 4.7).

\subsection{Anxiety}

\subsubsection{End of treatment}

Nine studies supplied data for the comparison of CBT to an active control (Abbott 2009; Arif 2017; Davies 1995; Hesser 2012; Jasper 2014; Malinvaud 2016; McKenna 2017; Philippot 2012a; Weise 2016). There was a significant difference in favour of CBT (SMD -0.25, 95\% Cl -0.48 to -0.02 ; 9 studies; 696 participants; Analysis 4.8) with moderate to substantial heterogeneity $\left(\mathrm{Chi}^{2}=16.54\right.$, df $\left.=8 ; \mathrm{P}=0.04 ; \mathrm{I}^{2}=52 \%\right)$. We rated the certainty of the evidence as low, which indicated that the true effect might be substantially different from the effect estimate (Summary of findings table 4).

\subsubsection{Six months follow-up}

At six months follow-up, McKenna 2017 reported that there were no differences between the MBCT group and active control (relaxation therapy) in HADS-A scores after pretreatment scores had been taken into consideration (MD $-1.20,95 \% \mathrm{Cl}-3.07$ to $0.67 ; 1$ study; 62 participants; Analysis 4.9).

\subsubsection{Twelve months follow-up}

No data were available for this outcome at this time point.

\subsection{Health-related quality of life}

\subsubsection{End of treatment}

One study reported quality of life data for the comparison of CBT and other active control (Hesser 2012), but found no difference between the conditions (MD -0.05, 95\% $\mathrm{Cl}-0.68$ to 0.59 ; 1 study, 95 participants; Analysis 4.10 ). We rated the certainty of the evidence for this outcome as very low, reflecting that we have little confidence in the effect estimate and that the true effect is likely to be substantially different (Summary of findings table 4).

4.5.2 Six months follow-up

No data were available for this outcome at this time point.

4.5.3 Twelve months follow-up

No data were available for this outcome at this time point.

\subsection{Negatively biased interpretations of tinnitus}

\subsubsection{End of treatment}

Data were supplied from five studies (Henry 1996; Hesser 2012; Jasper 2014; McKenna 2017; Weise 2016). These yielded results that found a significant difference in favour of CBT at the end of treatment (SMD $-0.55,95 \% \mathrm{Cl}-0.75$ to $-0.35 ; 5$ studies; 455 participants; Analysis 4.11). Heterogeneity might not be important $\left(\operatorname{Tau}^{2}=0.00\right.$; 
$\left.\mathrm{Chi}^{2}=4.23, \mathrm{df}=4(\mathrm{P}=0.38) ; \mathrm{I}^{2}=5 \%\right)$. We rated the certainty of the evidence for this outcome as moderate, indicating that the true effect is likely to be close to the effect estimate, but could possibly be substantially different (Summary of findings table 4).

\subsubsection{Six months follow-up}

At six months follow-up, McKenna 2017 reported that there was a statistically significant difference between participants' scores in the MBCT group compared to the active control (relaxation therapy) on catastrophizing as measured by the Tinnitus Catastrophizing Scale (TCS) (MD -7.20, 95\% Cl -13.65 to -0.75; 1 study; 62 participants; Analysis 4.12). McKenna 2017 reported an adjusted mean difference of $-4.6(95 \% \mathrm{Cl}$ -8.7 to -0.5$)$.

\subsubsection{Twelve months follow-up}

One study provided 12-month follow-up data that compared cognitive therapy combined with education, to education only, on the negatively biased interpretations of tinnitus as measured by the Tinnitus Cognitions Questionnaire (Henry 1996). Results from repeated measures ANOVAs indicated that there were no significant differences between the conditions.

\subsection{Other adverse effects}

\subsubsection{End of treatment}

No other adverse effects were reported at end of treatment.

\subsubsection{Six months follow-up}

No data were available for this outcome at this time point.

\subsubsection{Twelve months follow-up}

One study reported some slight deterioration in sleep quality in three participants (Weise 2016). (Note: by this time point there were no longer comparator participants since they had been offered and received CBT).

\section{Sensitivity analyses}

\section{Random-effects models compared to fixed-effect models}

There were no substantial differences between results depending on whether random-effects or fixed-effect modelling was used in the respective meta-analyses for the comparisons:

- $\quad$ CBT versus wait list control (Analysis 1.7; Analysis 1.8);

- CBT versus usual audiological care (Analysis 2.1; Analysis 2.7);

- $\quad$ CBT versus other active control (Analysis 4.14; Analysis 4.15).

\section{Excluding studies at high risk of bias for incomplete data}

Comparison 1: CBT versus no intervention/wait list control at end of treatment - impact of tinnitus on quality of life

We judged one study to be at high risk of bias for incomplete data (Malinvaud 2016). Retaining or excluding Malinvaud 2016 from the meta-analysis did not change the 
conclusion that CBT was more effective than wait list control, and did not change the effect size (moderate). With Malinvaud 2016 included the SMD was $-0.56(95 \% \mathrm{Cl}-0.83$ to $-0.30 ; 10$ studies, 537 participants; Analysis 1.1) and with Malinvaud 2016 excluded the SMD was -0.64 (95\% Cl -0.88 to $-0.40 ; 9$ studies, 454 participants; Analysis 1.15). Excluding Malinvaud 2016 from the analysis reduced the heterogeneity from $\mathrm{I}^{2}=49 \%$ $\left(\mathrm{Tau}^{2}=0.08 ; \mathrm{Chi}^{2}=17.62, \mathrm{df}=9(\mathrm{P}=0.04)\right)$ to $\mathrm{I}^{2}=28 \%\left(\mathrm{Tau}^{2}=0.04 ; \mathrm{Chi}^{2}=11.18, \mathrm{df}=8\right.$ $(P=0.19))$.

Comparison 4: CBT versus other active control at the end of treatment - impact of tinnitus on quality of life

We judged three studies to be at high risk of bias for incomplete outcome data (Abbott 2009; Davies 1995; Malinvaud 2016). With Abbott 2009, Davies 1995 and Malinvaud 2016 included the SMD was -0.32 (95\% Cl -0.56 to -0.08 ; 12 studies; 967 participants; Analysis 4.1), therefore lower than with the studies excluded (SMD $-0.48,95 \% \mathrm{Cl}-0.71$ to -0.26; 9 studies; 770 participants; Analysis 4.21). Excluding the studies did not change the finding that CBT was more effective at decreasing the impact of tinnitus on quality of life, but did lead to a change in the effect size from 'small' to 'moderate'. Statistical heterogeneity was also reduced when the three studies were excluded, from I $\mathrm{I}^{2}=66 \%$ $\left(\mathrm{Tau}^{2}=0.11 ; \mathrm{Chi}^{2}=32.28, \mathrm{df}=11(\mathrm{P}=0.0007)\right)$ to $\mathrm{I}^{2}=47 \%\left(\mathrm{Tau}^{2}=0.05 ; \mathrm{Chi}^{2}=15.18\right.$, $d f=8(P=0.06))$.

\section{Effects of replacing missing data with a 'conservative' compared with an 'optimistic' approach}

One study that was included in two comparisons had missing data that were replaced with (baseline) data showing no difference between the groups (Malinvaud 2016). To examine the effect of entering data on a 'conservative' basis showing no effect, we undertook a sensitivity analysis replacing it with data at three months follow-up, which showed a change in participants' scores in favour of CBT.

Comparison 1: CBT versus no intervention/wait list control at the end of treatment - impact of tinnitus on quality of life

Using data showing a more 'optimistic' response at the end of treatment resulted in a small increase in the SMD but did not change the effect size (moderate) or conclusion that CBT was more effective than wait list control at reducing the impact of tinnitus on quality of life at the end of treatment. Specifically, using the 'conservative' approach, the SMD was $-0.56(95 \% \mathrm{Cl}-0.83$ to -0.30 ; Analysis 1.1$)$ compared to the 'optimistic' scenario using three-month follow-up data (SMD -0.65, 95\% Cl-0.85 to -0.44; Analysis 1.16). Heterogeneity decreased from $\mathrm{I}^{2}=49 \%\left(\mathrm{Tau}^{2}=0.08 ; \mathrm{Chi}^{2}=17.62, \mathrm{df}=9, \mathrm{P}=0.04\right)$ to $\mathrm{I}^{2}=20 \%\left(\mathrm{Tau}^{2}=0.02 ; \mathrm{Chi}^{2}=11.29, \mathrm{df}=9, \mathrm{P}=0.26\right)$ with the use of three-month follow-up data.

Comparison 4: CBT versus other active control at the end of treatment - impact of tinnitus on quality of life

Using data that showed a more 'optimistic' response at the end of treatment resulted in a small increase in SMD score in favour of CBT, but did not change the effect size (small). Specifically, the SMD increased from $-0.32(95 \% \mathrm{Cl}-0.56$ to -0.08 ; Analysis 4.1$)$ 
to -0.37 ( $95 \% \mathrm{Cl}-0.58$ to -0.17 ; Analysis 4.23$)$ when the more 'optimistic' data were used. Furthermore, heterogeneity decreased from $\mathrm{I}^{2}=66 \%\left(\mathrm{Tau}^{2}=0.11 ; \mathrm{Chi}^{2}=32.28, \mathrm{df}=11\right.$, $\mathrm{P}=0.0007)$ to $\mathrm{I}^{2}=54 \%\left(\mathrm{Tau}^{2}=0.07 ; \mathrm{Chi}^{2}=23.74, \mathrm{df}=11, \mathrm{P}=0.01\right)$.

\section{Discussion}

\section{Summary of main results}

The objective of this review was to assess the effects and safety of cognitive behavioural therapy (CBT) for tinnitus in adults. Twenty-eight randomised controlled trials (RCTs) were included in the review with 21 of these supplying data for inclusion in metaanalyses. The four main comparisons of interest were wait list control, usual audiological care, tinnitus retraining therapy (TRT) and 'other' active control conditions.

Ten studies supplied data for the comparison of CBT with no intervention/wait list control. However, not all of them provided data on all the outcomes of interest for this review. There was evidence to indicate that CBT was superior to not providing any intervention in reducing the impact of tinnitus on quality of life and depression. However, there is limited evidence for CBT for tinnitus reducing anxiety, improving health-related quality of life, or reducing negatively biased interpretations of tinnitus (Summary of findings table 1). Furthermore, information from six of the 10 studies indicated that there were no serious adverse effects at the end of treatment. One study reported that one participant who received CBT experienced a deterioration in their symptoms. Although the results appear promising, effect sizes were small or medium, and the certainty of the evidence was very low, low or moderate, depending on the specific outcome.

Three studies supplied data for the comparison of CBT with audiological care. With regard to reducing the impact of tinnitus on quality of life, there was evidence to indicate that CBT was superior to audiological care and without serious adverse effects. The mean difference across the three studies (5.65) was less than the difference of seven points that has been reported to reflect a clinically important change (Zeman 2011). For this comparison though it is relevant to consider that one large study $(n=336)$ reported a large effect in favour of CBT and influences the overall result. There were negligible differences between CBT and audiological care on measures of depression, anxiety or quality of life. There was insufficient evidence to conclude that either CBT or audiological care is superior or inferior with regard to reducing negatively biased interpretations of tinnitus. No other adverse effects occurred. (See Summary of findings table 2).

There was insufficient evidence to conclude that CBT is superior or inferior to TRT on the outcomes of interest as only one study was included for this comparison (Westin 2011). In one of the few examples of studies reporting adverse effects, Westin 2011 found that three participant's tinnitus worsened following treatment (one from the CBT and two from the TRT group). (See Summary of findings table 3).

Twelve studies compared CBT to another active control condition. Across the studies there was variation in what form of CBT was used, what the comparison group received, 
whether it was individual or group treatment, delivered in person or not, and who delivered the treatment(s). Despite this, there was evidence to indicate that overall and albeit with small effect sizes, CBT is superior to other active treatments (e.g. relaxation, information sessions) in reducing the impact of tinnitus on quality of life. Similarly, small effect sizes in favour of CBT were found for reducing symptoms of depression, anxiety and negatively biased interpretations of tinnitus. Data from six of the 12 studies also suggest that adverse effects were less likely with CBT than with other active treatments. There was insufficient evidence to support the superiority or inferiority of CBT compared to other active treatments on quality of life. (See Summary of findings table 4).

Sensitivity analyses indicated that the findings and conclusions were robust to tests of assumptions and the methods used.

\section{Results from subgroup analyses}

The subgroup analyses examined whether there were differences in effects between: the types of CBT; modes of delivery; unit of delivery; health professional involved in delivery; and whether effects differed between studies that excluded participants according to hearing thresholds. Results from each of these analyses are briefly discussed below.

\section{Type of CBT}

Subgroup analyses for both the comparison of CBT versus wait list control and an active control intervention (excluding usual audiological care and TRT) found no statistically significant differences between the types of CBT (Analysis 1.7; Analysis 4.14). It is important to note, however, that the most frequent intervention type included in the subgroup analyses was CBT.

In summary, the type of CBT intervention used might not matter, but a relatively low number of studies using mindfulness or acceptance and commitment therapy (ACT) (i.e. 'third wave' CBT) prevents more definitive conclusions from being drawn.

Mode of delivery

While there was no statistically significant difference between the effects of CBT when mode of delivery was taken into account, there was greater variation in effect size when CBT was delivered face-to-face compared with as an Internet-based intervention. If CBT delivered as an Internet-based intervention is indeed as effective as face-to-face CBT, this has implications for access to treatment and cost-effectiveness. (See Implications for practice).

\section{Unit of delivery}

Subgroup analyses in the respective comparisons examining whether there were difference in effectiveness of CBT delivered individually compared with groups were consistent in their results. They both found that there was no statistical difference when CBT was delivered individually compared to groups (Analysis 1.11; Analysis 4.17). 
Health professional involved in delivery

There were no statistically significant differences in effect between who (or what) delivered CBT when comparing CBT to wait list (Analysis 1.13) or an 'other' active control (Analysis 4.18). Two issues are important to consider. First, the Internet-delivered and bibliotherapy CBT interventions were designed by psychologists but were not delivered by psychologists. Secondly, it was unclear exactly in what training or profession the "other clinicians" were in four studies across the two subgroup analyses (Andersson 2005; Arif 2017; Jakes 1992; Kreuzer 2012). These issues prevent conclusions being drawn with regard to the question of whether it matters who delivers CBT.

Hearing thresholds as a selection criteria in RCTs of CBT for tinnitus

Only one study included in this review excluded participants with severe hearing loss (Westin 2011). On one hand, this means that it is not possible to establish whether there are differences in results obtained between studies that examine whether there are differences in effectiveness of CBT depending on hearing loss, but on the other hand, it means that the results from the review can be applied to all people seeking help for tinnitus-related distress regardless of whether or not they have hearing loss.

\section{Additional results and considerations}

Many articles did not report or collect data on adverse effects or other outcomes of interest

Adverse effects of CBT for tinnitus are rare. However, this conclusion comes with the caveat that relatively few studies actually reported whether adverse effects occurred or not, and when they did there was little or no information on the method used to identify adverse effects. The exception to this being in four studies (Beukes 2018a; Nyenhuis 2013a; Weise 2016; Westin 2011), where deterioration on measures of tinnitus-related quality of life was established using the calculation of reliable change indices. When not reported, information about adverse effects was sought from and/or obtained from personal email communication with authors involved in the studies. Although a lot of authors responded to our inquiries, not all were able to recall whether there were adverse effects or not.

In addition to the absence of data relating to adverse effects, it was relatively rare for studies to collect outcome data for quality of life (6 of 28 studies) and negatively biased interpretations of tinnitus (10 of 28 studies).

Few studies collected six- and 12-month follow-up data

Collecting longer-term follow-up data is vital for examining whether or not any intervention effects are sustained over time. This in turn can have an effect on treatment preferences of the patient, service provider and at a policy level. The time points of interest for this review were post-treatment, six- and 12-month follow-up, which in the context of 'longer-term follow-up' is not actually that long. Unfortunately, only two studies reported outcome data that were collected at six months and six studies collected outcome data at 12 months follow-up. Even then, not all the data collected at these time points could be included in meta-analyses as the comparators were mostly wait list control conditions and participants had already received CBT by the six- or 
12-month time point. The average duration for which follow-up data were collected was four months and the median was three months.

Short-term follow-up data might also limit the ability of multi-item questionnaires to detect changes in overall quality of life in particular. This might be the case here because a change in one domain (e.g. tinnitus-related quality of life) might not immediately generalise or have an effect on other domains of life in the short term, but might do so in the longer term.

Origin of CBT materials

Viktor Kaldo and Gerhard Andersson co-authored a manual (in Swedish) for conducting CBT for tinnitus (Kaldo 2004a), which subsequently formed the basis for many bibliotherapy and Internet-based interventions. Nine of the 22 studies that supplied data for meta-analyses are derived from this work. In addition to the Swedish studies (Andersson 2002; Andersson 2005; Hesser 2012; Kaldo 2007), the treatment manual and Internet-based interventions have been adapted, translated and used in English(Abbott 2009; Beukes 2018a; Beukes 2018b) and German-speaking populations (Jasper 2014; Weise 2016). With the exception of Abbott 2009, which used a cluster-randomised control design and had a high participant dropout rate, results from the other eight studies were consistently in favour of CBT over comparison conditions. This suggests that the CBT content delivered with little human interaction is efficacious and robust to different contexts and languages.

Issues of sample size

With the exception of one study, all studies involved samples of fewer than 150 participants. Cima 2012 included 492 participants. This one large study reported a large effect size in favour of CBT and was weighted highly in the meta-analysis for the comparison in which it was included (CBT compared to audiological care). Ultimately the result showing that CBT was superior to audiological treatment was thus largely determined by the results of Cima 2012.

Outlier study

In general the results of studies included in this review found that CBT is superior to other control/comparator conditions with regard to reducing the impact tinnitus has on quality of life. The notable exception to this was Oron (unpublished), which found that the participants in the comparator intervention (coping effectiveness training; CET) had significantly lower scores on the THI than participants in the acceptance and commitment therapy (ACT) condition. A possible explanation for this result could be that CET appears to have some intervention components that are commonly found in CBT interventions. For example, CET included problem-solving skills with the intention of reducing stress, relaxation and pleasant activity scheduling.

Clinical significance

All of the included studies report group-level statistical significance of changes in the outcomes of the interventions. However, only 15 studies discussed clinical significance. Reporting the clinical significance of an intervention is important in order to provide an 
indication of size of effect, and the numbers of individual participants who might have benefited, remained the same or deteriorated following treatment. Clinical significance can be calculated in many ways and there is no standardised criteria or method for doing this. For example, within the included studies Kaldo 2007 used a pre-specified criteria of a $50 \%$ reduction in the Tinnitus Reaction Questionnaire (TRQ) score as a sign of clinically significant change. In contrast, Weise 2016 used two methods to estimate clinical significance. Firstly, the Reliable Change Index (Jacobson 1991) to establish the change in score required to reflect change beyond measurement error, and second, calculating the numbers of participants who were "highly functioning" following treatment according to if they scored in the "mild" range on the THI (i.e. less than or equal to 36$)$.

Given that there were only small to moderate effect sizes achieved, it seems that there is still room for improvement in the design and implementation of CBT interventions for tinnitus. However, by way of comparison, the magnitudes of the effect sizes reported in this review are similar to those reported for psychological interventions for treating chronic pain (Ecclestone 2013), which has many conceptual similarities to chronic tinnitus.

Studies at high risk of bias for missing data affected heterogeneity

Sensitivity analyses revealed that including studies judged to be at high risk for missing data inflated the level of heterogeneity (Abbott 2009; Davies 1995; Malinvaud 2016). Furthermore, for Comparison 1, the inclusion of Malinvaud 2016 also contributed to us downgrading the level of evidence for inconsistency in the results - that is, inconsistency that we introduced ourselves into the data set by including the study.

\section{Limitations}

The results of this review should be interpreted with five main limitations in mind. First, information about adverse effects associated with CBT was rarely reported, and the methods associated with monitoring or seeking information about adverse effects were not reported. Where information about adverse effects (serious or otherwise) was not reported, we made efforts to contact authors to obtain this information. Not every author we contacted responded to our requests for information and in many cases, information was dependent on recall of studies that occurred many years ago. It is conceivable that authors who responded to our inquiries were less likely to have observed adverse effects in their studies, thus leading to an underestimation of the risks associated with CBT. Nonetheless, CBT is considered to be a safe intervention, compared to surgical and pharmacological treatments since it is non-invasive.

A second limitation within this review concerns the lack of six- and 12-month follow-up data. Very few studies collected longer-term follow-up data, which in turn limits our ability to draw any conclusions about the longer-term efficacy of CBT for tinnitus. This has long been recognised as an issue within tinnitus research (Henry 1996), but little has changed in recent years.

'Risk of bias' assessments revealed that there were very few studies at low risk of performance and detection biases. This is partially a product of CBT as intervention 
being difficult to mask from participants and that trialists depended on multi-item selfreport questionnaires of subjective variables such as tinnitus-related quality of life. None of the included studies had, for example, an independent outcome assessor to complement the responses obtained from the self-report questionnaires.

Another limitation within the review is the limited number of studies included for the subgroup analyses: those results should be interpreted with caution.

Lastly, although not an inherent limitation, we only included evidence from parallelgroup and cluster-randomised controlled trials. Excluding non-randomised studies may limit the generalisability of drawing conclusions on how well CBT for tinnitus will work in everyday clinical practice. Although accompanied by higher risks of bias, the (potential) benefit of non-randomised trials is that they can inform judgments of the effectiveness of CBT when implemented in everyday practice.

\section{Overall completeness and applicability of evidence}

\section{Completeness}

The completeness of the evidence was variable with regard to the four main comparisons included in this review. There is sufficient evidence of moderate certainty to conclude that CBT is superior to wait list control conditions at the end of treatment. However, there were too few studies comparing CBT with audiological care and TRT respectively to reach a conclusion. For the comparison of CBT with any other active intervention, the rating of the evidence (i.e. low certainty) means that our confidence in the effect estimate is limited.

Information about adverse effects (serious or otherwise) was rarely reported (although we obtained information about adverse effects from some authors). Three-quarters of the studies provided data for inclusion in meta-analyses of the primary outcome (impact of tinnitus on quality of life). Outcome data for depression and anxiety were more frequently reported than data related to quality of life and negatively biased interpretations of tinnitus.

Across all comparisons and outcomes there was an absence of evidence of the efficacy of CBT at 6 or 12 months after treatment.

For the subgroup analyses, there were considerable differences in the number of studies that provided data within the respective comparisons. For example, there was a comparable number of studies using different modes and units of delivery, but not of types of therapy, professional delivering therapy or of studies using and not using hearing loss as an exclusion criteria.

\section{Applicability}

There was a relatively complete representation of the population of people living with and seeking psychological help for tinnitus. There was a wide age range, a wide range of tinnitus duration, and a balance of males and females. Only one study excluded 
participants based on hearing thresholds (Westin 2011). The most frequent exclusion criteria were objective tinnitus and/or severe mental health conditions. In summary, the evidence appears applicable to people suffering from chronic tinnitus. People suffering from tinnitus and seeking pharmacological, electrical or electromagnetic stimulation therapy, or bio- neuro-feedback treatments would not have sought to participate in the included studies, and thus could be different in some characteristics.

Between the studies there was considerable variation in the possible combinations and permutations of CBT; that is, there was variation in the type of CBT, how it was delivered to participants, the unit of delivery, who delivered CBT and the duration of treatment. With the exception of the studies linked to Andersson and Kaldo's work there was also variation in the content of the CBT interventions included/used in the studies. The planned comparisons we specified meant that almost all RCTs of CBT for tinnitus have been included, making the results applicable to CBT for tinnitus in general.

The results from this review are most applicable to when either a psychologist (or psychologist in training) delivers the interventions and/or designed the CBT intervention. Although 'therapists' were referred to in some studies there was, overall, an absence of evidence from studies that had professionals other than psychologists delivering CBT. The absence of follow-up data also means that the results are only applicable to CBT in the short rather than medium or long term.

It is also important to recognise that the data included in this review came from studies conducted in outpatient clinics in a relatively small number of European countries, America and Australia. One study from China was included in the review, but did not provide data suitable for inclusion in meta-analyses. We are thus unclear about the effectiveness of CBT for tinnitus when conducted in inpatient settings or in other countries beyond those included here. Similarly, it is unclear what effects CBT might have when delivered by professionals such as audiologists due to an absence of data.

\section{Quality of the evidence}

For the comparison 'CBT versus no intervention/wait list control' the certainty of evidence for the two primary outcomes was low and moderate respectively. We downgraded the evidence (10 studies) by one level due to risk of bias associated with an absence of blinding of participants and personnel with regard to both the intervention and outcome assessment. We also downgraded the evidence for the outcome impact of tinnitus on quality of life by one level due to inconsistency in the results.

For the comparison 'CBT versus audiological care' the certainty of evidence for the primary outcomes was moderate. We downgraded the evidence (three studies) by one level due to risk of bias. We judged all studies in this comparison to be at high risk of performance bias due to an absence of blinding of participants and personnel.

For the comparison 'CBT versus tinnitus retraining therapy' we rated the certainty of evidence for the primary outcomes as low. We downgraded the evidence (one study) primarily due to high risk of bias associated with an absence of allocation concealment, 
and also an unclear risk of bias due to performance, detection and attrition biases. We also downgraded the evidence by one level due to imprecision (sample size fewer than 350 participants).

For the comparison 'CBT versus other experimental control' we rated the certainty of evidence for the primary outcomes as low. For the outcome of impact of tinnitus on quality of life we downgraded the evidence by two levels due to risk of bias (unclear or high risk associated with performance bias and blinding) and inconsistency (not all confidence levels overlapped and there was high statistical heterogeneity). For the outcome of serious adverse effects, we downgraded the evidence by two levels due to unclear or high risk of performance and attrition bias respectively.

\section{Potential biases in the review process}

The searches of the electronic databases were conducted independently of the group of authors by Information Specialists within the Cochrane ENT group. We also searched the reference lists of the included studies and previous Cochrane Reviews (MartinezDevesa 2010). Date of publication and language were not barriers to inclusion in this review. In addition to English, we reviewed full-text articles in Chinese and German for eligibility assessment. Where authors of this review were listed as authors of included studies, they were not involved in decisions related to screening, data extraction or risk of bias assessment.

We conscientiously followed Cochrane guidelines and the methods described in the protocol (Fuller 2017b). This, however, does not entirely prevent biases from influencing the outcome of the review. It is possible that biases influenced decisions in the development of the protocol and thus the procedures that we followed thereafter. For example, how we defined CBT determined the studies that were included. There is some debate about whether ACT and mindfulness constitute a new form of CBT or should be considered independently (Hofmann 2008). Similarly, after some debate within the group, we excluded Malouff 2010 on the grounds that giving participants a book based on CBT principles, but without any contact from a psychologist or other therapist, does not constitute CBT. It is possible that a different group of authors might have reached different conclusions about these issues.

There were three minor changes to the roles of authors in relation to data extraction and risk of bias assessments (see Differences between protocol and review). However, given that this led to additional participation from more rather than fewer authors, it is likely that biased thinking was decreased rather than increased as a result of greater scrutiny amongst the review team.

In this review, one cluster-randomised controlled trial was included (Abbott 2009). The analysis conducted by Abbott 2009 did not take into account the clustered nature of the data, and the corresponding author informed us that the data set was no longer available. The Cochrane Handbook for Systematic Reviews of Interventions advises that a post-hoc calculation can be made to adjust the sample size to account for the clustered nature of the data, using a value for the intracluster correlation (ICC) (Higgins 
2011). If an ICC is not reported in the article, a suitable alternative should be identified. Ultimately, we used an ICC value from a cluster-RCT with hearing aid users as the best/closest approximation (Meijerink 2017). This ICC was identified by the Cochrane Methods Support Unit (see Acknowledgements) and the decision to use it was taken in consultation with the Cochrane ENT Managing Editor.

\section{Agreements and disagreements with other studies or reviews}

The conclusions of this review are mostly consistent with previous systematic reviews. The previous Cochrane Review of CBT for tinnitus concluded that "CBT has a positive effect on the management of tinnitus" (p.2; Martinez-Devesa 2010). This conclusion was based on results showing significant improvement in the impact of tinnitus on quality of life and depression scores. While we agree with this conclusion in principle, our results are more nuanced as a result of being able to include more recent studies and subsequently conduct subgroup analyses. Furthermore our conclusions about the efficacy of CBT are limited to post-treatment as there is an absence of evidence at six and 12 months follow-up.

The effect sizes for the impact of tinnitus on quality of life reported for CBT versus wait list control at end of treatment in Martinez-Devesa 2010 were larger (standardised mean difference (SMD) 0.91, 95\% confidence interval (Cl) 0.50 to 1.32; 5 studies; 309 participants) than the ones we report (SMD $0.56,95 \% \mathrm{Cl}-0.83$ to $-0.30 ; 10$ studies; 537 participants). We identified a few examples of participant deterioration following CBT, though Martinez-Devesa 2010 did not.

Regarding the secondary outcomes, for depression our results for the comparison of CBT versus wait list control and versus another active treatment were in agreement. Specifically, each review reported medium and small effect sizes respectively. MartinezDevesa 2010, however, did not examine outcomes related to anxiety, quality of life or negatively biased interpretations of tinnitus. We on the other hand did not include subjective loudness of tinnitus as an outcome despite it being the primary outcome of Martinez-Devesa 2010. Research has consistently reported that CBT does not affect the perceptual characteristics of tinnitus.

While our conclusions are consistent with Martinez-Devesa 2010 there are differences in the studies that were included and excluded. We excluded the following studies that Martinez-Devesa 2010 included: Kröner-Herwig 1995; Kröner-Herwig 2003; Rief 2005; Weise 2008; Zachriat 2004. (See Excluded studies for reasons why the studies were excluded from this review). In contrast, we included the following studies that MartinezDevesa 2010 excluded: Abbott 2009; Andersson 2002; Davies 1995; Henry 1998; Jakes 1986; Jakes 1992; Lindberg 1989; Robinson 2008. Five of these studies were excluded by Martinez-Devesa 2010 due to high participant dropout rates, a criterion that is no longer considered as valid for exclusion. One study was excluded for not having usable data (Henry 1998), one for not being randomised (Jakes 1986), and one study was judged not to have used CBT as an intervention (Lindberg 1989). We included these studies although none provided data that could be included in the meta-analyses. 
Two studies were listed in Martinez-Devesa 2010 as ongoing studies at the time of publication. We included one, Schmidt 2018 (referred to as "Kendall 2009" in MartinezDevesa 2010, p. 12) and excluded one, Zenner 2013 (referred to as "Zenner 2010" in Martinez-Devesa 2010, p. 12).

In addition to the previous Cochrane Review of CBT for tinnitus, there have been nine other systematic reviews that have examined the efficacy of CBT for tinnitus (Andersson 1999; Hesser 2011; Hoare 2011b; Nyenhuis 2013b; Cima 2014; Beukes 2019; Landry 2019; Mehta 2019; Rademaker 2019). Although these reviews differ from each other and our review in their focus and methodology, the conclusions are consistent in that CBT appears to be superior to other control conditions for alleviating the impact of tinnitus on quality of life. The moderate effect sizes for CBT versus wait list control reported by Hesser 2011 and Hoare 2011b are comparable with our results but smaller than the large effect size reported by Landry 2019.

\section{Authors' conclusions}

\section{Implications for practice}

There are six main implications for practice generated by this review:

1. The main results of this review indicate that cognitive behavioural therapy (CBT) may be effective in reducing the impact of tinnitus on quality of life at the end of treatment, and that there are few if any adverse effects from receiving CBT (although further research on this is recommended below). These results provide further evidence or justification for recommendations made in two prominent clinical guidelines endorsing the provision of CBT for patients with chronic bothersome tinnitus (Cima 2019; Tunkel 2014). Consequently, policy-makers and service providers should feel confident that CBT for tinnitus is beneficial for patients at least in the short term. This is not to say, however, that CBT is an easy form of treatment to engage in; it is often personally challenging and can require a considerable investment of time and money from the patient (assuming that CBT is even available and/or covered by insurance in a given country).

2. CBT for tinnitus appears to have some benefit for people who also experience depression, but the effects are small and there are some concerns with regards to the quality of the evidence. Thus, in addition to receiving tinnitus-specific CBT, people with co-morbid depression should also seek depression-specific treatment. Overall, there is either low-certainty evidence, small effects and/or an insufficient amount of evidence currently to recommend CBT for tinnitus if the primary intention is to improve anxiety or general quality of life, or to change negatively biased interpretations of tinnitus.

3. CBT for tinnitus delivered in person and delivered via the Internet, with some additional email communication from a professional, appear similarly effective, as does CBT delivered individually and group-wise. Alternative modes of delivery should be considered depending on patient preference, accessibility and cost. 
4. There is insufficient evidence to support a recommendation for whom should provide CBT for tinnitus, although it is noted that psychologists and/or psychiatrists were involved in the design, conduct and/or supervision of all CBT treatments.

5. The results from this review are relevant to tinnitus patients with varying levels of hearing loss and thus they should also be eligible to access treatment. We do not know, however, to what extent the study populations represent the whole patient population.

6. It is important to keep in mind that approximately half of the included studies in the review only reported group-level data/analyses. This means that the results represent an average of the outcomes for participants in the study. In other words, on average, people improved receiving CBT compared with waiting for it (tinnitus) to get better, or another available treatment. It is likely that individual patients might respond better or worse than the average treatment effects reported here and that patients should make informed choices aligned with personal preferences where possible.

\section{Implications for research}

Future research into the effectiveness of CBT for tinnitus should use the most rigorous methods available. Researchers can do this at least in part by using the SPIRIT statement when designing study protocols (Chan 2013), pre-registering trials at sites (such as clinicaltrials.gov, osf.io or aspredicted.org) and using the CONSORT statement to guide reporting of the results from the study (Schulz 2010). Using reporting guidelines and pre-registering trials can help ensure transparency in the conduct and reporting of studies and potentially increase confidence in the results and help future systematic reviewers make decisions with regard to whether a particular study meets eligibility criteria for inclusion. In this review only 10 of the 28 included studies were pre-registered, only one protocol was published ahead of the study and one protocol fully published as a supplementary file. While the aforementioned regards the general planning and reporting of a study, researchers should in future make efforts to assess adverse effects, report how they did so and record whether adverse effects occurred. Researchers should also give greater consideration to assessment of outcomes and use complementary methods to self-report questionnaires (e.g. blinded outcome assessors; healthcare use; multi-item self-report questionnaires completed by carers or partners of people receiving treatment). This would reduce some of the risks of bias associated with outcome assessment.

Recommendations for future research include the following:

1. Use follow-up measurement points of at least 6 to 12 months in order to assess the longer-term efficacy of CBT for tinnitus. Presently there is insufficient evidence available to comment on the efficacy of CBT at these time points. Researchers could also investigate the impact that 'booster' sessions might have in enhancing any of the effects from treatment and/or delaying or preventing relapse from occurring.

2. Use pre-specified primary outcomes including serious adverse effects. If multiple primary outcome measures are to be used, a rationale for doing so should be included. Ongoing work is currently being conducted to establish a (minimum) core set of outcome measures (Hall 2018), which should be included in all future studies in order to improve evidence synthesis and the ability to compare results between 
studies. We urge researchers to keep informed of future recommendations resulting from this work.

3. Systematically examine components of CBT interventions or compare specific CBT protocols, in order to reduce heterogeneity and further refine treatment protocols.

4. Ensure that a power analysis is conducted to inform the target sample size for a study and report the results. If the target sample size is not reached this should be reported as well as a discussion on how this affected the power of the study and interpretation of results.

5. Examine the efficacy of CBT delivered by healthcare professionals other than psychologists (or trainee psychologists). If other health professionals can provide effective CBT for tinnitus, then there would be scope to increase access to treatment.

6. Establish which form of treatment works best for whom, especially since there is considerable heterogeneity in tinnitus. Randomised controlled trials (RCTs) are not typically designed to examine this issue and thus they would either need to be specifically designed to do so and/or other methods should be used. An alternative research method, which is increasingly used to inform such decisions, is the single case experimental design (Schork 2015). Single case experimental design studies have high internal validity and require fewer participants and resources to conduct.

7. Establish differences in effectiveness between types of CBT. This issue should be investigated in RCTs, single case experimental trials and future systematic reviews.

8. Ensure that estimates of clinical significance are included in the results of studies.

9. Incorporate treatment fidelity checks into RCTs to verify that interventions are delivered as intended. This is particularly relevant and important if the same therapists are delivering two or more interventions in the same study in order to assess if there is 'contamination' or insufficient 'protocol adherence' in the interventions.

\section{Acknowledgements}

We would like to thank the corresponding authors of the following included studies for providing additional information: Abbott 2009; Andersson 2002; Andersson 2005; Arif 2017; Beukes 2018a; Beukes 2018b; Cima 2012; Kaldo 2007; Lindberg 1989; Malinvaud 2016; Martz 2018; Oron (unpublished); Philippot 2012a; Robinson 2008; Schmidt 2018; Westin 2011. We would also like to thank the corresponding authors of Kröner-Herwig 1995, Kröner-Herwig 1999, Kröner-Herwig 2003, Kröner-Herwig 2006 and Tucker 2013, who provided additional information about their studies that ultimately helped inform our decisions on whether they met the inclusion criteria.

We would like to acknowledge Jenny Bellorini for all the practical assistance and guidance throughout the review. We thank Samantha Cox for preparing and conducting the initial and final literature searches, and Vittoria Lutje for conducting an update search. We would also like to thank Aidan Tan and Yu-Tian Xiao who extracted data from and conducted the risk of bias assessment for the Zhong 2014 study. 
We would like to thank Nuala Livingstone and Kerry Dwan from the Cochrane Methods Support Unit for providing guidance and an intracluster correlation coefficient to enable data from Abbott 2009 to be included in meta-analyses.

Thomas Fuller was supported by SWOL Limburgs Fonds voor Revalidatie and the Netherlands Organisation for Health Research and Development (ZonMW), Research programme: Health Care Efficiency, Subprogramme: Effects \& Costs, Grant number: 945-07-715.

Rilana Cima received funding from the Innovational Research Incentives Scheme Veni, from the Netherlands Organisation for Scientific Research (NWO).

Derek $\mathrm{J}$ Hoare is funded through the National Institute for Health Research (NIHR) Biomedical Research Centre Programme. The view expressed are those of the author and not necessarily those of the NIHR, the NHS, or the Department of Health and Social Care.

Johan WS Vlaeyen received funding from the Research Foundation, Flanders FWO, Belgium (Fonds Wetenschappelijk Onderzoek Vlaanderen) and the Netherlands Organisation for Health Research and Development (ZonMW).

This project was supported by the National Institute for Health Research, via Cochrane Infrastructure, Cochrane Programme Grant or Cochrane Incentive funding to Cochrane ENT. The views and opinions expressed therein are those of the authors and do not necessarily reflect those of the Systematic Reviews Programme, NIHR, NHS or the Department of Health.

\section{Contributions of authors}

The author contributions were as follows:

- TF, RC and JWSV conceived and all authors contributed to the design of the study.

- The Cochrane ENT Information Specialist developed and ran the search strategy.

- TF obtained copies of the studies with the assistance of Maastricht University Library.

- TF, RC and DH were responsible for the selection of studies.

- TF, RC, BM and DH were responsible for data extraction.

- $\mathrm{TF}, \mathrm{RC}, \mathrm{BM}$ and $\mathrm{DH}$ were responsible for assessing risk of bias.

- TF entered data into RevMan.

- TF, RC and DH, in consultation with Cochrane Methods Support Unit, conducted the analysis.

- All authors contributed to the interpretation of the analysis.

- TF drafted and revised the review and all authors commented critically on intellectual content.

- All authors will contribute to the drafting and updating of the review. 


\section{Declarations of interest}

Where review authors were also study authors, they had no involvement in study selection, data extraction or risk of bias assessment.

Thomas Fuller: none known.

Rilana Cima: was an investigator and author of the Cima 2012 study, which was a RCT comparing stepped CBT-based care with treatment as usual on the impact of tinnitus on quality of life.

Berthold Langguth: has received funding for research from the Deutsche Forschungsgemeinschaft, the German Ministry for Research, the American Tinnitus Association, the Tinnitus Research Initiative, the European Union, Otonomy and Sivantos. He has received consultancy and speaker honoraria from Autifony, ANM, Astra Zeneca, Kyorin, Merz, McKinsey, Microtransponder, Neuromod, Novartis, Pfizer, Lundbeck and Servier.

Birgit Mazurek: has received funding for research from the Deutsche Forschungsgemeinschaft, the German Ministry for Research, the American Tinnitus Association and the German Tinnitus Association Charité.

Derek J Hoare: is vice chair of the British Society of Audiology.

Johan WS Vlaeyen: was an investigator and author of the Cima 2012 study, which was a RCT comparing stepped CBT-based care with treatment as usual on the impact of tinnitus on quality of life.

\section{Differences between protocol and review}

"Tinnitus reactivity", defined as being tinnitus-specific health-related quality of life, as measured by multi-item questionnaires (e.g. TFI), was specified as the primary outcome in our protocol (Fuller 2017b). This terminology was replaced in the review with "impact of tinnitus on quality of life" in order to be consistent with other reviews related to tinnitus in the Cochrane ENT group (Sereda 2018; Wegner 2018).

We had not specified in our protocol what outcome measure we would use if studies used more than one multi-item questionnaire to measure the secondary outcomes for depression, anxiety, quality of life and negatively biased interpretations of tinnitus. Whenever possible we chose the multi-item questionnaire that would allow pooling of data and analysis using mean differences. Failing that, we chose the measure that had the better psychometric properties. 
In the protocol we had not clearly described how we would treat cluster-RCTs that did not include information about the intracluster correlation coefficient (ICC). We followed the recommendations in Chapter 16.3.4 'Approximate analyses of cluster-randomised trials for a meta-analysis: effective sample sizes' in the Cochrane Handbook for Systematic Reviews of Interventions (Higgins 2011). After a search for examples of cluster-RCTs with tinnitus patients yielded no results, we contacted the Cochrane ENT group Managing Editor for further guidance. Courtesy of the Cochrane Methods Support Unit, we obtained an estimate of an ICC that was reasonable for us to use with this population. We also conducted a sensitivity analysis including and excluding the data from Abbott 2009.

We have provided some clarification in the report of the review regarding 'mindfulness'. We have added the following text: "We considered interventions as 'mindfulness' if they involved: exercises that involved self-regulation of attention on experience and emphasised openness, curiosity and acceptance (Bishop 2004)".

In the protocol we stated that if the $\mathrm{I}^{2}$ was $>30 \%$, the $\mathrm{Chi}^{2}$ value was greater than the degrees of freedom and/or the confidence intervals of the included studies did not show overlap, we would not pool studies and instead describe the findings in a narrative form. Although the $\mathrm{I}^{2}$ values were above $30 \%$ in numerous analyses, there was clear overlap of almost all the confidence intervals, and the trend of the effect across studies was consistently towards the interventions favouring CBT regardless of the comparator. Furthermore, we expected that the underlying reason for this high level of statistical heterogeneity was the anticipated clinical or methodological factors for which we had planned subgroup analyses. Given this and the potential value of a meta-analysis compared with only providing a narrative summary of the literature, we pooled the data.

We conducted an additional sensitivity analysis to examine the effect of using a 'conservative' compared to an 'optimistic' approach in one study (Malinvaud 2016), which did not report outcome data at the end of treatment but did so at three months follow-up.

Compared with what we initially described in the protocol, there were changes in the contributions of authors to two tasks. DH also contributed to data extraction, and BM and $\mathrm{RC}$ also conducted 'Risk of bias' assessments. AW withdrew from the review team due to increased work commitments elsewhere.

\section{Published notes}

The publication of this review will lead to the previous one becoming obsolete. A link to the superseded review will be available (Martinez-Devesa 2010).

\section{Characteristics of studies}

Details of characteristics of included, excluded and ongoing studies are provided in: Chapter 3_Supplementary Information File 


\section{Summary of findings tables}

Summary of Findings Table 1. CBT Compared to No Intervention/Waiting List Control for Tinnitus

\section{CBT compared to no intervention/waiting list control for tinnitus at end of treatment}

Patient or population: adults with tinnitus

Settings: online, hospitals, psychology department, self-help (with phone calls)

Intervention: CBT

Comparison: no intervention/waiting list control

\begin{tabular}{|c|c|c|c|c|c|c|}
\hline \multirow[b]{2}{*}{$\begin{array}{l}\text { Outcomes } \\
\text { at end of } \\
\text { treatment }\end{array}$} & \multicolumn{2}{|c|}{$\begin{array}{l}\text { Anticipated absolute } \\
\text { effects* }(95 \% \mathrm{Cl})\end{array}$} & \multirow[b]{2}{*}{$\begin{array}{l}\text { Relative } \\
\text { effect } \\
\text { (95\% } \\
\text { Cl) }\end{array}$} & \multirow[b]{2}{*}{$\begin{array}{l}\text { № of } \\
\text { partici- } \\
\text { pants } \\
\text { (studies) }\end{array}$} & \multirow[b]{2}{*}{$\begin{array}{l}\text { Certainty } \\
\text { of the } \\
\text { evidence } \\
\text { (GRADE) }\end{array}$} & \multirow[b]{2}{*}{ Comments } \\
\hline & $\begin{array}{l}\text { Risk with no } \\
\text { intervention/ } \\
\text { waiting list } \\
\text { control }\end{array}$ & $\begin{array}{l}\text { Risk with } \\
\text { CBT }\end{array}$ & & & & \\
\hline $\begin{array}{l}\text { Impact of } \\
\text { tinnitus on } \\
\text { quality of life } \\
\text { at treatment } \\
\text { end } \\
\text { Assessed } \\
\text { with: TFI, } \\
\text { THI, TRQ, } \\
\text { TEQ }\end{array}$ & - & $\begin{array}{l}\text { SMD } 0.56 \\
\text { lower } \\
\text { ( } 0.83 \text { lower } \\
\text { to } 0.30 \\
\text { lower) }\end{array}$ & - & $\begin{array}{l}537 \\
(10 \\
\text { RCTs })\end{array}$ & $\begin{array}{l}\oplus \oplus \Theta \Theta \\
\text { low }^{1,2}\end{array}$ & $\begin{array}{l}\text { CBT may reduce } \\
\text { the impact of } \\
\text { tinnitus on quality } \\
\text { of life at treatment } \\
\text { end. } \\
\text { The SMD can be } \\
\text { interpreted as the } \\
\text { THI score in the } \\
\text { CBT group being } \\
\text { on average } 10.91 \\
\text { points lower than } \\
\text { in the no interven- } \\
\text { tion/waiting list } \\
\text { control group. (The } \\
\text { minimal clinically } \\
\text { important change } \\
\text { score has been } \\
\text { estimated to be } 7 \\
\text { points on the THI). }\end{array}$ \\
\hline
\end{tabular}


Summary of Findings Table 1. Continued.

\begin{tabular}{|c|c|c|c|c|c|c|}
\hline $\begin{array}{l}\text { Outcomes } \\
\text { at end of } \\
\text { treatment }\end{array}$ & $\begin{array}{l}\text { Anticipated abs } \\
\text { effects }(95 \% \mathrm{CI}) \\
\text { Risk with no in- } \\
\text { tervention/wait- } \\
\text { ing list control }\end{array}$ & $\begin{array}{l}\text { olute } \\
\text { Risk with } \\
\text { CBT }\end{array}$ & $\begin{array}{l}\text { Relative } \\
\text { effect } \\
(95 \% \mathrm{Cl})\end{array}$ & $\begin{array}{l}\text { № of par- } \\
\text { ticipants } \\
\text { (studies) }\end{array}$ & $\begin{array}{l}\text { Certainty } \\
\text { of the } \\
\text { evidence } \\
\text { (GRADE) }\end{array}$ & Comments \\
\hline \multirow{2}{*}{$\begin{array}{l}\text { Serious ad- } \\
\text { verse effects } \\
\text { at end of } \\
\text { treatment }\end{array}$} & \multicolumn{2}{|c|}{ Study population } & \multirow{2}{*}{$\begin{array}{l}\text { RR } 3.00 \\
(0.13 \text { to } \\
69.87)\end{array}$} & \multirow{2}{*}{$\begin{array}{l}447 \\
\text { (7 RCTs) }\end{array}$} & \multirow{2}{*}{$\begin{array}{l}\oplus \oplus \oplus \Theta \\
\text { moderate }^{1}\end{array}$} & One participant \\
\hline & 0 per 1000 & $\begin{array}{l}0 \text { per } 1000 \\
(0 \text { to } 0)\end{array}$ & & & & $\begin{array}{l}\text { allocated to CBT } \\
\text { deteriorated. How- } \\
\text { ever, the deteriora- } \\
\text { tion in symptoms } \\
\text { occurred between } \\
\text { two assessments } \\
\text { prior to the inter- } \\
\text { vention commenc- } \\
\text { ing but was still } \\
\text { detectable at end } \\
\text { of treatment. } \\
\text { CBT probably } \\
\text { results in little or } \\
\text { no difference in } \\
\text { adverse effects. }\end{array}$ \\
\hline $\begin{array}{l}\text { Depression } \\
\text { at end of } \\
\text { treatment } \\
\text { Assessed } \\
\text { with: } \\
\text { BDI, } \\
\text { HADS-D, } \\
\text { PHQ-9 }\end{array}$ & - & $\begin{array}{l}\text { SMD } 0.34 \\
\text { lower } \\
\text { (0.60 lower } \\
\text { to } 0.08 \\
\text { lower) }\end{array}$ & - & $\begin{array}{l}502 \\
\text { (8 RCTs) }\end{array}$ & $\begin{array}{l}\oplus \oplus \Theta \Theta \\
\text { low }^{1,2}\end{array}$ & $\begin{array}{l}\text { CBT may result in } \\
\text { a slight reduction } \\
\text { in depression at } \\
\text { end of treatment. }\end{array}$ \\
\hline $\begin{array}{l}\text { Anxiety at } \\
\text { end of treat- } \\
\text { ment } \\
\text { Assessed } \\
\text { with: } \\
\text { HADS-A, } \\
\text { GAD-7 }\end{array}$ & - & $\begin{array}{l}\text { SMD } 0.45 \\
\text { lower } \\
\text { (0.82 lower } \\
\text { to } 0.09 \\
\text { lower) }\end{array}$ & - & $\begin{array}{l}429 \\
\text { (6 RCTs) }\end{array}$ & $\begin{array}{l}\oplus \Theta \Theta \Theta \\
\text { very } \\
\text { low }^{1,3,4}\end{array}$ & $\begin{array}{l}\text { The evidence is } \\
\text { very uncertain } \\
\text { about whether } \\
\text { CBT reduces } \\
\text { anxiety at end of } \\
\text { treatment. }\end{array}$ \\
\hline $\begin{array}{l}\text { Health-re- } \\
\text { lated quality } \\
\text { of life } \\
\text { Assessed } \\
\text { with: } \\
\text { SWLS, QoLI }\end{array}$ & - & $\begin{array}{l}\text { SMD } 0.38 \\
\text { lower } \\
\text { (0.67 lower } \\
\text { to } 0.08 \\
\text { lower) }\end{array}$ & - & $\begin{array}{l}179 \\
\text { (2 RCTs) }\end{array}$ & $\begin{array}{l}\oplus \Theta \Theta \Theta \\
\text { very } \\
\text { low }^{5,6,7}\end{array}$ & $\begin{array}{l}\text { The evidence is } \\
\text { very uncertain } \\
\text { about whether } \\
\text { CBT improves } \\
\text { health-related } \\
\text { quality of life. }\end{array}$ \\
\hline
\end{tabular}


Summary of Findings Table 1. Continued.

\begin{tabular}{|c|c|c|c|c|c|c|}
\hline \multirow[b]{2}{*}{$\begin{array}{l}\text { Outcomes } \\
\text { at end of } \\
\text { treatment }\end{array}$} & \multicolumn{2}{|c|}{$\begin{array}{l}\text { Anticipated absolute } \\
\text { effects" }(95 \% \mathrm{Cl})\end{array}$} & \multirow[b]{2}{*}{$\begin{array}{l}\text { Relative } \\
\text { effect } \\
(95 \% \mathrm{Cl})\end{array}$} & \multirow{2}{*}{$\begin{array}{l}\text { № of par- } \\
\text { ticipants } \\
\text { (studies) }\end{array}$} & \multirow{2}{*}{$\begin{array}{l}\text { Certainty } \\
\text { of the } \\
\text { evidence } \\
\text { (GRADE) }\end{array}$} & \multirow[b]{2}{*}{ Comments } \\
\hline & $\begin{array}{l}\text { Risk with no in- } \\
\text { tervention/wait- } \\
\text { ing list control }\end{array}$ & $\begin{array}{l}\text { Risk with } \\
\text { CBT }\end{array}$ & & & & \\
\hline $\begin{array}{l}\text { Negatively } \\
\text { biased inter- } \\
\text { pretations of } \\
\text { tinnitus } \\
\text { Assessed } \\
\text { with: TAQ,TCQ }\end{array}$ & - & $\begin{array}{l}\text { SMD } 0.4 \\
\text { lower } \\
(1.25 \text { lower } \\
\text { to } 0.45 \\
\text { higher })\end{array}$ & - & $\begin{array}{l}84 \\
(2 \mathrm{RCTs})\end{array}$ & $\begin{array}{l}\oplus \Theta \Theta \Theta \\
\text { very } \\
\text { low }^{2,7,8}\end{array}$ & $\begin{array}{l}\text { The evidence is } \\
\text { very uncertain } \\
\text { about whether CBT } \\
\text { reduces negatively } \\
\text { biased interpreta- } \\
\text { tions of tinnitus. }\end{array}$ \\
\hline $\begin{array}{l}\text { Other ad- } \\
\text { verse effects }\end{array}$ & No adverse effec & ts occurred. & - & $\begin{array}{l}447 \\
\text { (7 RCTs) }\end{array}$ & $\begin{array}{l}\oplus \oplus \oplus \Theta \\
\text { moderate }^{1}\end{array}$ & - \\
\hline
\end{tabular}

${ }^{*}$ The risk in the intervention group (and its 95\% confidence interval) is based on the assumed risk in the comparison group and the relative effect of the intervention (and its 95\% Cl).

BDI: Beck Depression Inventory; CBT: cognitive behavioural therapy; CI: confidence interval; GAD-7: Generalized Anxiety Disorder-7; HADS-A: Hospital Anxiety and Depression Scale-Anxiety; HADS-D: Hospital Anxiety and Depression Scale-Depression; PHQ-9: Patient Health Questionnaire; QoLI: Quality of Life Inventory; RCT: randomised controlled trial; RR: risk ratio; SWLS: Satisfaction With Life Survey; TAQ: Tinnitus Acceptance Questionnaire; TCQ: Tinnitus Cognitions Questionnaire.; TEQ: Tinnitus Effects Questionnaire; TFI: Tinnitus Functional Inventory; THI: Tinnitus Handicap Inventory; TRQ: Tinnitus Reaction Questionnaire

GRADE Working Group grades of evidence

High certainty: We are very confident that the true effect lies close to that of the estimate of the effect Moderate certainty: We are moderately confident in the effect estimate: The true effect is likely to be close to the estimate of the effect, but there is a possibility that it is substantially different Low certainty: Our confidence in the effect estimate is limited: The true effect may be substantially different from the estimate of the effect

Very low certainty: We have very little confidence in the effect estimate: The true effect is likely to be substantially different from the estimate of effect

\section{Footnotes}

${ }^{1}$ Downgraded one level due to study limitations (risk of bias): all studies included for this outcome were judged to be either unclear or at high risk of performance bias due to an absence of blinding of participants and personnel.

${ }^{2}$ Downgraded one level due to inconsistency: significant heterogeneity in the studies.

${ }^{3}$ Downgraded one level due to study limitations (risk of bias): two studies with a weighting of $36 \%$ were judged to be at high risk of selection bias.

${ }^{4}$ Downgraded one level due to inconsistency: confidence intervals of two studies did not overlap and the $\mathrm{I}^{2}$ value was $67 \%$.

${ }^{5}$ Downgraded one level due to study limitations (risk of bias): studies judged to be at high risk of selection bias.

${ }^{6}$ Downgraded one level due to study limitations (risk bias): studies judged to be at unclear or high risk of performance and detection bias. 
${ }^{7}$ Downgraded one level due to imprecision: small sample size.

${ }^{8}$ Downgraded one level due to study limitations (risk of bias): one study judged to be at high risk of selection bias and both were judged to be at uncertain risk of performance and detection biases.

Summary of Findings Table 2. CBT Compared to Audiological Care (Tinnitus Education and Rehabilitation for Hearing Loss) for Tinnitus

\section{CBT compared to audiological care (tinnitus education and rehabilitation for hearing} loss) for tinnitus at end of treatment

Patient or population: adults with tinnitus

Settings: audiological rehabilitation centre, hospital, Veterans Affairs, online Intervention: CBT

Comparison: audiological care (tinnitus education and rehabilitation for hearing loss)

\begin{tabular}{|c|c|c|c|c|c|c|}
\hline \multirow[b]{2}{*}{$\begin{array}{l}\text { Outcomes at } \\
\text { end of } \\
\text { treatment }\end{array}$} & \multicolumn{2}{|c|}{$\begin{array}{l}\text { Anticipated absolute } \\
\text { effects }^{*}(95 \% \mathrm{Cl})\end{array}$} & \multirow[b]{2}{*}{$\begin{array}{l}\text { Relative } \\
\text { effect } \\
(95 \% \mathrm{Cl})\end{array}$} & \multirow[b]{2}{*}{$\begin{array}{l}\text { № of par- } \\
\text { ticipants } \\
\text { (studies) }\end{array}$} & \multirow[b]{2}{*}{$\begin{array}{l}\text { Certainty } \\
\text { of the } \\
\text { evidence } \\
\text { (GRADE) }\end{array}$} & \multirow[b]{2}{*}{ Comments } \\
\hline & $\begin{array}{l}\text { Risk with } \\
\text { audiological } \\
\text { care (tinnitus } \\
\text { education and } \\
\text { rehabilitation } \\
\text { for hearing } \\
\text { loss) }\end{array}$ & $\begin{array}{l}\text { Risk with } \\
\text { CBT }\end{array}$ & & & & \\
\hline $\begin{array}{l}\text { Impact of } \\
\text { tinnitus on } \\
\text { quality of life } \\
\text { Assessed } \\
\text { with: Tinnitus } \\
\text { Handicap } \\
\text { Inventory } \\
\text { Scale from: } 0 \\
\text { to } 100\end{array}$ & 34.14 & $\begin{array}{l}\text { MD } 5.65 \\
\text { lower } \\
\text { ( } 9.79 \text { lower } \\
\text { to } 1.5 \\
\text { lower) }\end{array}$ & - & $\begin{array}{l}430 \\
\text { (3 RCTs) }\end{array}$ & $\begin{array}{l}\oplus \oplus \oplus \Theta \\
\text { moder- } \\
\text { ate }^{1}\end{array}$ & $\begin{array}{l}\text { The MD is } \\
\text { reported here } \\
\text { because the } 3 \\
\text { studies all re- } \\
\text { ported outcome } \\
\text { data from the } \\
\text { THI. } \\
\text { CBT probably } \\
\text { reduces the } \\
\text { impact of tinnitus } \\
\text { on quality of life } \\
\text { when compared } \\
\text { with audiological } \\
\text { care. }\end{array}$ \\
\hline $\begin{array}{l}\text { Serious ad- } \\
\text { verse effects }\end{array}$ & $\begin{array}{l}\text { No serious adve } \\
\text { occurred. }\end{array}$ & rse effects & - & $\begin{array}{l}410 \\
(2 \mathrm{RCTs})\end{array}$ & $\begin{array}{l}\oplus \oplus \oplus \Theta \\
\text { moder- } \\
\text { ate }^{1}\end{array}$ & $\begin{array}{l}\text { Meta-analysis } \\
\text { was not con- } \\
\text { ducted for this } \\
\text { outcome. }\end{array}$ \\
\hline $\begin{array}{l}\text { Depression at } \\
\text { end of treat- } \\
\text { ment } \\
\text { Assessed } \\
\text { with: HADS-D, } \\
\text { PHQ-9 }\end{array}$ & - & $\begin{array}{l}\text { SMD } 0.18 \\
\text { lower } \\
\text { ( } 0.38 \text { lower } \\
\text { to } 0.01 \\
\text { higher) }\end{array}$ & - & $\begin{array}{l}410 \\
\text { (2 RCTs) }\end{array}$ & $\begin{array}{l}\oplus \oplus \Theta \Theta \\
\text { low }^{1,2}\end{array}$ & $\begin{array}{l}\text { CBT may slightly } \\
\text { reduce depres- } \\
\text { sion at end of } \\
\text { treatment when } \\
\text { compared with } \\
\text { audiological } \\
\text { care. }\end{array}$ \\
\hline
\end{tabular}


Summary of Findings Table 2. Continued.

\begin{tabular}{|c|c|c|c|c|c|c|}
\hline \multirow[b]{2}{*}{$\begin{array}{l}\text { Outcomes at } \\
\text { end of } \\
\text { treatment }\end{array}$} & \multicolumn{2}{|c|}{$\begin{array}{l}\text { Anticipated absolute } \\
\text { effects }^{*}(95 \% \mathrm{Cl})\end{array}$} & \multirow[b]{2}{*}{$\begin{array}{l}\text { Relative } \\
\text { effect } \\
\text { (95\% CI) }\end{array}$} & \multirow[b]{2}{*}{$\begin{array}{l}\text { № of par- } \\
\text { ticipants } \\
\text { (studies) }\end{array}$} & \multirow[b]{2}{*}{$\begin{array}{l}\text { Certainty } \\
\text { of the } \\
\text { evidence } \\
\text { (GRADE) }\end{array}$} & \multirow[b]{2}{*}{ Comments } \\
\hline & $\begin{array}{l}\text { Risk with } \\
\text { audiological } \\
\text { care (tinnitus } \\
\text { education and } \\
\text { rehabilitation } \\
\text { for hearing loss) } \\
\end{array}$ & $\begin{array}{l}\text { Risk with } \\
\text { CBT }\end{array}$ & & & & \\
\hline $\begin{array}{l}\text { Anxiety at end } \\
\text { of treatment } \\
\text { Assessed } \\
\text { with: GAD-7, } \\
\text { HADS-A }\end{array}$ & - & $\begin{array}{l}\text { SMD } 0.06 \\
\text { lower } \\
(0.26 \text { lower } \\
\text { to } 0.13 \\
\text { higher) }\end{array}$ & - & $\begin{array}{l}410 \\
(2 \mathrm{RCTs})\end{array}$ & $\begin{array}{l}\oplus \oplus \Theta \Theta \\
\text { low }^{1,2}\end{array}$ & $\begin{array}{l}\text { CBT may result in } \\
\text { little to no differ- } \\
\text { ence in anxiety at } \\
\text { end of treatment } \\
\text { when compared } \\
\text { with audiological } \\
\text { care. }\end{array}$ \\
\hline $\begin{array}{l}\text { Health-related } \\
\text { quality of life } \\
\text { Assessed } \\
\text { with: HUI, } \\
\text { SWLS }\end{array}$ & - & $\begin{array}{l}\text { SMD } 0.07 \\
\text { lower } \\
(0.26 \text { lower } \\
\text { to } 0.13 \\
\text { higher) }\end{array}$ & - & $\begin{array}{l}410 \\
(2 \mathrm{RCTs})\end{array}$ & $\begin{array}{l}\oplus \oplus \Theta \Theta \\
\text { low }^{1,2}\end{array}$ & $\begin{array}{l}\text { CBT may result in } \\
\text { little to no differ- } \\
\text { ence in health-re- } \\
\text { lated quality of life } \\
\text { when compared } \\
\text { with audiological } \\
\text { care. }\end{array}$ \\
\hline $\begin{array}{l}\text { Negatively } \\
\text { biased inter- } \\
\text { pretations of } \\
\text { tinnitus } \\
\text { Assessed } \\
\text { with: TCS } \\
\text { Scale from: } 0 \\
\text { to } 65\end{array}$ & $\begin{array}{l}\text { At end of } \\
\text { treatment TCS } \\
\text { scores had } \\
\text { decreased from } \\
\text { a mean of } 21 . \\
42 \text { (SD 12.56) } \\
\text { to } 17.14 \text { (SD } \\
11.54) .\end{array}$ & $\begin{array}{l}\text { At end of } \\
\text { treatment } \\
\text { TCS } \\
\text { scores had } \\
\text { decreased } \\
\text { from a } \\
\text { mean of } \\
20.89 \text { (SD } \\
11.83 \text { ) } \\
\text { to } 12.45 \\
(10.30) .\end{array}$ & - & $\begin{array}{l}336 \\
(1 \mathrm{RCT})\end{array}$ & $\begin{array}{l}\oplus \oplus \Theta \Theta \\
\text { low }^{3,4}\end{array}$ & $\begin{array}{l}\text { CBT may reduce } \\
\text { negatively biased } \\
\text { interpretations } \\
\text { of tinnitus when } \\
\text { compared with } \\
\text { audiological care. }\end{array}$ \\
\hline $\begin{array}{l}\text { Other adverse } \\
\text { effects }\end{array}$ & No adverse effec & ts occurred. & - & $\begin{array}{l}410 \\
\text { (2 RCTs) }\end{array}$ & $\begin{array}{l}\oplus \oplus \oplus \Theta \\
\text { moderate }^{1}\end{array}$ & - \\
\hline
\end{tabular}

${ }^{\star}$ The risk in the intervention group (and its 95\% confidence interval) is based on the assumed risk in the comparison group and the relative effect of the intervention (and its $95 \% \mathrm{Cl}$ ). The assumed risk score in the comparison group (34.14) was obtained from the median control group score from the largest study (Cima 2012) in this comparison.

CBT: cognitive behavioural therapy; CI: confidence interval; GAD-7: Generalized Anxiety Disorder-7; HADS-A: Hospital Anxiety and Depression Scale-Anxiety; HADS-D: Hospital Anxiety and Depression Scale-Depression; HUI: Health Utilities Index; MD: mean difference; PHQ-9: Patient Health Questionnaire; RCT: randomised controlled trial; RR: risk ratio; SWLS: Satisfaction With Life Survey; TCS: Tinnitus Catastrophizing Scale

GRADE Working Group grades of evidence

High certainty: We are very confident that the true effect lies close to that of the estimate of 
the effect

Moderate certainty: We are moderately confident in the effect estimate: The true effect is likely to be close to the estimate of the effect, but there is a possibility that it is substantially different Low certainty: Our confidence in the effect estimate is limited: The true effect may be substantially different from the estimate of the effect

Very low certainty: We have very little confidence in the effect estimate: The true effect is likely to be substantially different from the estimate of effect.

\section{Footnotes}

'Downgraded one level due to study limitations (risk of bias): all studies included for this outcome were judged to be either unclear or at high risk of performance bias due to an absence of blinding of participants and personnel.

2Downgraded by one level due to imprecision: the confidence intervals cross the line of no effect. ${ }^{3}$ Downgraded one level due to study limitations (risk of bias): performance and detection bias judged as unclear.

${ }^{4}$ Downgraded one level due to imprecision: small sample size. 
Chapter 3

Summary of Findings Table 3. CBT Compared to TRT (Directive Counselling and Bilateral Masking) for Tinnitus

CBT compared to TRT (directive counselling and bilateral masking) for tinnitus at end of treatment

Patient or population: adults with tinnitus

Setting: hospital

Intervention: CBT

Comparison: TRT (directive counselling and bilateral masking)

\begin{tabular}{|c|c|c|c|c|c|c|}
\hline \multirow[b]{2}{*}{$\begin{array}{l}\text { Outcomes } \\
\text { at end of } \\
\text { treatment }\end{array}$} & \multicolumn{2}{|c|}{$\begin{array}{l}\text { Anticipated absolute } \\
\text { effects* }(95 \% \mathrm{Cl})\end{array}$} & \multirow[b]{2}{*}{$\begin{array}{l}\text { Relative } \\
\text { effect } \\
(95 \% \mathrm{Cl})\end{array}$} & \multirow[b]{2}{*}{$\begin{array}{l}\text { № of par- } \\
\text { ticipants } \\
\text { (studies) }\end{array}$} & \multirow[b]{2}{*}{$\begin{array}{l}\text { Certainty } \\
\text { of the } \\
\text { evidence } \\
\text { (GRADE) }\end{array}$} & \multirow[b]{2}{*}{ Comments } \\
\hline & $\begin{array}{l}\text { Risk with TRT } \\
\text { (directive } \\
\text { counselling } \\
\text { and bilateral } \\
\text { masking) }\end{array}$ & $\begin{array}{l}\text { Risk with } \\
\text { CBT }\end{array}$ & & & & \\
\hline $\begin{array}{l}\text { Impact of } \\
\text { tinnitus on } \\
\text { quality of life } \\
\text { Assessed } \\
\text { with: } \text { THI } \\
\text { Scale from: } 0 \\
\text { to } 100\end{array}$ & $\begin{array}{l}\text { At } 10 \text { weeks } \\
\text { the THI score } \\
\text { had decreased } \\
\text { from an aver- } \\
\text { age of } 47.00 \\
\text { (SD 18.19) to } \\
\text { an average } \\
\text { of } 43.22 \text { (SD } \\
20.75 \text { ). }\end{array}$ & $\begin{array}{l}\text { At } 10 \\
\text { weeks the } \\
\text { THI score } \\
\text { had de- } \\
\text { creased } \\
\text { from an } \\
\text { average } \\
\text { of } 45.27 \\
\text { (SD 14.99) } \\
\text { to an } \\
\text { average } \\
\text { of } 27.43 \\
\text { (19.18). }\end{array}$ & - & $\begin{array}{l}42 \\
(1 \mathrm{RCT})\end{array}$ & $\begin{array}{l}\oplus \oplus \Theta \Theta \\
\text { low }^{1,2}\end{array}$ & $\begin{array}{l}\text { CBT may reduce } \\
\text { the impact of } \\
\text { tinnitus on quality } \\
\text { of life compared } \\
\text { with TRT. }\end{array}$ \\
\hline \multirow{2}{*}{$\begin{array}{l}\text { Serious ad- } \\
\text { verse effects }\end{array}$} & \multicolumn{2}{|c|}{ Study population } & RR 0.45 & & $\oplus \oplus \Theta \Theta$ & Three participants \\
\hline & 100 per 1000 & $\begin{array}{l}45 \text { per } \\
1000 \\
\text { (4 to } 464)\end{array}$ & $\begin{array}{l}(0.04 \text { to } \\
4.64)\end{array}$ & (1 RCT) & $\operatorname{low}^{1,2}$ & $\begin{array}{l}\text { deteriorated over } \\
\text { the course of the } \\
\text { study: } 1 \text { partici- } \\
\text { pant was from the } \\
\text { intervention group } \\
\text { (ACT; } n=22 \text { ) and } \\
2 \text { participants } \\
\text { were from the } \\
\text { comparison group } \\
\text { (TRT; } n=20 \text { ). }\end{array}$ \\
\hline
\end{tabular}


Summary of Findings Table 3. Continued.

\begin{tabular}{|c|c|c|c|c|c|c|}
\hline \multirow[b]{2}{*}{$\begin{array}{l}\text { Outcomes } \\
\text { at end of } \\
\text { treatment }\end{array}$} & \multicolumn{2}{|c|}{$\begin{array}{l}\text { Anticipated absolute } \\
\text { effects }^{*}(95 \% \mathrm{Cl})\end{array}$} & \multirow[b]{2}{*}{$\begin{array}{l}\text { Relative } \\
\text { effect } \\
(95 \% \mathrm{Cl})\end{array}$} & \multirow[b]{2}{*}{$\begin{array}{l}\text { № of par- } \\
\text { ticipants } \\
\text { (studies) }\end{array}$} & \multirow[b]{2}{*}{$\begin{array}{l}\text { Certainty } \\
\text { of the } \\
\text { evidence } \\
\text { (GRADE) }\end{array}$} & \multirow[b]{2}{*}{ Comments } \\
\hline & $\begin{array}{l}\text { Risk with TRT } \\
\text { (directive } \\
\text { counselling } \\
\text { and bilateral } \\
\text { masking) } \\
\end{array}$ & $\begin{array}{l}\text { Risk with } \\
\text { CBT }\end{array}$ & & & & \\
\hline $\begin{array}{l}\text { Depression } \\
\text { Assessed } \\
\text { with: } \\
\text { HADS-D } \\
\text { Scale from: } 0 \\
\text { to } 21\end{array}$ & $\begin{array}{l}\text { At } 10 \text { weeks } \\
\text { the HADS-D } \\
\text { scores had de- } \\
\text { creased from } \\
\text { a mean of } 5.80 \\
\text { (SD 3.79) to } \\
5.78 \text { (SD 3.73). }\end{array}$ & $\begin{array}{l}\text { At } 10 \\
\text { weeks the } \\
\text { HADS-D } \\
\text { scores } \\
\text { had de- } \\
\text { creased } \\
\text { from a } \\
\text { mean of } \\
4.05 \text { (SD } \\
3.06 \text { ) to } \\
3.20 \text { (SD } \\
3.47) \text {. }\end{array}$ & - & $\begin{array}{l}42 \\
(1 \mathrm{RCT})\end{array}$ & $\begin{array}{l}\oplus \oplus \Theta \Theta \\
\text { low }^{1,2}\end{array}$ & $\begin{array}{l}\text { We are uncertain } \\
\text { whether CBT } \\
\text { reduces depres- } \\
\text { sion compared } \\
\text { with TRT. }\end{array}$ \\
\hline $\begin{array}{l}\text { Anxiety } \\
\text { Assessed } \\
\text { with: } \\
\text { HADS-A } \\
\text { Scale from: } 0 \\
\text { to } 21\end{array}$ & $\begin{array}{l}\text { At } 10 \text { weeks } \\
\text { the HADS-A } \\
\text { scores had de- } \\
\text { creased from } \\
\text { a mean of } 8.2 \\
\text { (SD 3.75) to } 7.0 \\
\text { (SD 4.20). }\end{array}$ & $\begin{array}{l}\text { At } 10 \\
\text { weeks the } \\
\text { HADS-A } \\
\text { scores } \\
\text { had de- } \\
\text { creased } \\
\text { from a } \\
\text { mean of } \\
6.24 \text { (SD } \\
4.00 \text { ) to } \\
3.6 \text { (SD } \\
3.14) \text {. }\end{array}$ & - & $\begin{array}{l}42 \\
(1 \mathrm{RCT})\end{array}$ & $\begin{array}{l}\oplus \oplus \Theta \Theta \\
\text { low }^{1,2}\end{array}$ & $\begin{array}{l}\text { We are uncertain } \\
\text { whether CBT } \\
\text { reduces anxiety } \\
\text { compared with } \\
\text { TRT. }\end{array}$ \\
\hline $\begin{array}{l}\text { Health-re- } \\
\text { lated quality } \\
\text { of life } \\
\text { Assessed } \\
\text { with: QoLI } \\
\text { Scale from: } \\
-6 \text { to } 6\end{array}$ & $\begin{array}{l}\text { At } 10 \text { weeks } \\
\text { QoLI scores } \\
\text { had increased } \\
\text { from a mean of } \\
2.24 \text { (SD 1.42) } \\
\text { to } 2.47 \text { (SD } \\
1.72 \text { ). }\end{array}$ & $\begin{array}{l}\text { At } 10 \\
\text { weeks } \\
\text { QoLI } \\
\text { scores } \\
\text { had } \\
\text { increased } \\
\text { from a } \\
\text { mean of } \\
2.43 \text { (SD } \\
1.30 \text { ) to } \\
2.78 \text { (SD } \\
1.53) \text {. }\end{array}$ & - & $\begin{array}{l}42 \\
(1 \mathrm{RCT})\end{array}$ & $\begin{array}{l}\oplus \oplus \Theta \Theta \\
\text { low }^{1,2}\end{array}$ & $\begin{array}{l}\text { We are uncertain } \\
\text { whether CBT im- } \\
\text { proves health-re- } \\
\text { lated quality of } \\
\text { life compared } \\
\text { with TRT. }\end{array}$ \\
\hline
\end{tabular}


Summary of Findings Table 3. Continued.

\begin{tabular}{|c|c|c|c|c|c|c|}
\hline \multirow[b]{2}{*}{$\begin{array}{l}\text { Outcomes } \\
\text { at end of } \\
\text { treatment }\end{array}$} & \multicolumn{2}{|c|}{$\begin{array}{l}\text { Anticipated absolute } \\
\text { effects }^{*}(95 \% \mathrm{Cl})\end{array}$} & \multirow[b]{2}{*}{$\begin{array}{l}\text { Relative } \\
\text { effect } \\
(95 \% \mathrm{Cl})\end{array}$} & \multirow[b]{2}{*}{$\begin{array}{l}\text { № of par- } \\
\text { ticipants } \\
\text { (studies) }\end{array}$} & \multirow[b]{2}{*}{$\begin{array}{l}\text { Certainty } \\
\text { of the } \\
\text { evidence } \\
\text { (GRADE) }\end{array}$} & \multirow[b]{2}{*}{ Comments } \\
\hline & $\begin{array}{l}\text { Risk with TRT } \\
\text { (directive } \\
\text { counselling } \\
\text { and bilateral } \\
\text { masking) }\end{array}$ & $\begin{array}{l}\text { Risk with } \\
\text { CBT }\end{array}$ & & & & \\
\hline $\begin{array}{l}\text { Negatively } \\
\text { biased inter- } \\
\text { pretations of } \\
\text { tinnitus } \\
\text { Assessed } \\
\text { with: TAQ } \\
\text { Scale from: } 0 \\
\text { to } 72\end{array}$ & $\begin{array}{l}\text { At } 10 \text { weeks } \\
\text { TAQ scores } \\
\text { had increased } \\
\text { from a mean } \\
\text { of } 36.65 \text { (9.96) } \\
\text { to } 37.89 \text { (SD } \\
10.73) \text {. }\end{array}$ & $\begin{array}{l}\text { At } 10 \\
\text { weeks } \\
\text { TAQ } \\
\text { scores } \\
\text { had } \\
\text { increased } \\
\text { from a } \\
\text { mean of } \\
41.05 \text { (SD } \\
9.49 \text { ) to } \\
47.67 \text { (SD } \\
11.15) \text {. }\end{array}$ & - & $\begin{array}{l}42 \\
(1 \mathrm{RCT})\end{array}$ & $\begin{array}{l}\oplus \oplus \Theta \Theta \\
\text { low }^{1,2}\end{array}$ & $\begin{array}{l}\text { CBT may reduce } \\
\text { negatively biased } \\
\text { interpretations of } \\
\text { tinnitus compared } \\
\text { with TRT. }\end{array}$ \\
\hline $\begin{array}{l}\text { Other ad- } \\
\text { verse effects }\end{array}$ & $\begin{array}{l}\text { No other advers } \\
\text { were reported. }\end{array}$ & se effects & - & $\begin{array}{l}42 \\
(1 \mathrm{RCT})\end{array}$ & $\begin{array}{l}\oplus \oplus \Theta \Theta \\
\text { low }^{1,2}\end{array}$ & - \\
\hline
\end{tabular}

${ }^{*}$ The risk in the intervention group (and its 95\% confidence interval) is based on the assumed risk in the comparison group and the relative effect of the intervention (and its $95 \% \mathrm{Cl}$ ).

ACT: acceptance and commitment therapy; CI: confidence interval; HADS-A: Hospital Anxiety and Depression Scale-Anxiety; HADS-D: Hospital Anxiety and Depression Scale-Depression; QoLI: Quality of Life Inventory; RCT: randomised controlled trial; RR: risk ratio; SD: standard deviation; TAQ: Tinnitus Acceptance Questionnaire; THI: Tinnitus Handicap Inventory; TRT: tinnitus retraining therapy

GRADE Working Group grades of evidence

High certainty: We are very confident that the true effect lies close to that of the estimate of the effect Moderate certainty: We are moderately confident in the effect estimate: The true effect is likely to be close to the estimate of the effect, but there is a possibility that it is substantially different Low certainty: Our confidence in the effect estimate is limited: The true effect may be substantially different from the estimate of the effect

Very low certainty: We have very little confidence in the effect estimate: The true effect is likely to be substantially different from the estimate of effect

\section{Footnotes}

'Downgraded one level due to study limitations (risk of bias). There was high risk of bias associated with allocation concealment and unclear risk of bias for performance, detection and attrition bias respectively.

${ }^{2}$ Downgraded one level due to imprecision: small sample size. 
Summary of Findings Table 4. CBT Compared to Other Experimental Control for Tinnitus

\section{CBT compared to other experimental control for tinnitus}

Patient or population: adults with tinnitus

Settings: hospital, online

Intervention: CBT

Comparison: other experimental control

\begin{tabular}{|c|c|c|c|c|c|c|}
\hline $\begin{array}{l}\text { Outcomes } \\
\text { at end of } \\
\text { treatment }\end{array}$ & $\begin{array}{l}\text { Anticipated ab } \\
\text { effects }^{*}(95 \% \text { C } \\
\text { Risk with oth- } \\
\text { er experimen- } \\
\text { tal control }\end{array}$ & $\begin{array}{l}\text { solute } \\
\text { Il) } \\
\text { Risk with } \\
\text { CBT }\end{array}$ & $\begin{array}{l}\text { Relative } \\
\text { effect } \\
(95 \% \mathrm{Cl})\end{array}$ & $\begin{array}{l}\text { № of par- } \\
\text { ticipants } \\
\text { (studies) }\end{array}$ & $\begin{array}{l}\text { Certainty } \\
\text { of the } \\
\text { evidence } \\
\text { (GRADE) }\end{array}$ & Comments \\
\hline $\begin{array}{l}\text { Impact of } \\
\text { tinnitus on } \\
\text { quality of } \\
\text { life at end of } \\
\text { treatment } \\
\text { Assessed } \\
\text { with: TFI, THI, } \\
\text { THQ, TQ, } \\
\text { TRQ, TEQ-ED }\end{array}$ & - & $\begin{array}{l}\text { SMD } 0.30 \\
\text { lower } \\
\text { (0.55 low- } \\
\text { er to } 0.05 \\
\text { lower) }\end{array}$ & - & $\begin{array}{l}966 \\
\text { (12 RCTs) }\end{array}$ & $\begin{array}{l}\oplus \oplus \Theta \Theta \\
\text { low }^{1,2}\end{array}$ & $\begin{array}{l}\text { CBT may reduce } \\
\text { the impact of } \\
\text { tinnitus on quality } \\
\text { of life when com- } \\
\text { pared with other } \\
\text { treatments. } \\
\text { The SMD can be } \\
\text { interpreted as } \\
\text { the THI score in } \\
\text { the CBT group } \\
\text { being on average } \\
5.84 \text { points lower } \\
\text { than in the other } \\
\text { experimental con- } \\
\text { trol group. (The } \\
\text { minimal clinically } \\
\text { important change } \\
\text { score has been } \\
\text { estimated to be } 7 \\
\text { points on the THI). }\end{array}$ \\
\hline \multirow{2}{*}{$\begin{array}{l}\text { Serious ad- } \\
\text { verse effects }\end{array}$} & \multicolumn{2}{|l|}{ Study population } & RR 1.70 & 595 & $\oplus \oplus \Theta \Theta$ & Three participants \\
\hline & 6 per 1000 & $\begin{array}{l}10 \text { per } \\
1000 \\
(1 \text { to } 105)\end{array}$ & $\begin{array}{l}\text { (0.16 to } \\
18.36)\end{array}$ & (6 RCTs) & $\operatorname{low}^{1,3}$ & $\begin{array}{l}\text { deteriorated ac- } \\
\text { cording to reliable } \\
\text { change calcula- } \\
\text { tions using the } \\
\text { TQ; } 1 \text { was from } \\
\text { the group CBT } \\
\text { intervention and } 2 \\
\text { received "informa- } \\
\text { tion only" control. }\end{array}$ \\
\hline
\end{tabular}


Summary of Findings Table 4. Continued.

\begin{tabular}{|c|c|c|c|c|c|c|}
\hline $\begin{array}{l}\text { Outcomes } \\
\text { at end of } \\
\text { treatment }\end{array}$ & $\begin{array}{l}\text { Anticipated abs } \\
\text { effects }{ }^{*}(95 \% \mathrm{C} \\
\text { Risk with oth- } \\
\text { er experimen- } \\
\text { tal control }\end{array}$ & $\begin{array}{l}\text { Risk with } \\
\text { CBT }\end{array}$ & $\begin{array}{l}\text { Relative } \\
\text { effect } \\
(95 \% \mathrm{Cl})\end{array}$ & $\begin{array}{l}\text { № of par- } \\
\text { ticipants } \\
\text { (studies) }\end{array}$ & $\begin{array}{l}\text { Certainty } \\
\text { of the } \\
\text { evidence } \\
\text { (GRADE) }\end{array}$ & Comments \\
\hline $\begin{array}{l}\text { Depression } \\
\text { at end of } \\
\text { treatment } \\
\text { Assessed } \\
\text { with: BDI, } \\
\text { DASS, } \\
\text { HADS-D, } \\
\text { HRSD, PHQ-9 }\end{array}$ & - & $\begin{array}{l}\text { SMD } 0.17 \\
\text { lower } \\
\text { (0.33 low- } \\
\text { er to } 0.01 \\
\text { lower) }\end{array}$ & - & $\begin{array}{l}943 \\
(11 \mathrm{RCTs})\end{array}$ & $\begin{array}{l}\oplus \oplus \Theta \Theta \\
\text { low }^{1,4}\end{array}$ & $\begin{array}{l}\text { CBT may reduce } \\
\text { depression when } \\
\text { compared with } \\
\text { other treatments. }\end{array}$ \\
\hline $\begin{array}{l}\text { Anxiety at } \\
\text { end of treat- } \\
\text { ment } \\
\text { Assessed } \\
\text { with: DASS, } \\
\text { HADS-A, } \\
\text { STAI }\end{array}$ & - & $\begin{array}{l}\text { SMD } 0.25 \\
\text { lower } \\
(0.48 \text { low- } \\
\text { er to } 0.02 \\
\text { lower) }\end{array}$ & - & $\begin{array}{l}696 \\
\text { (9 RCTs) }\end{array}$ & $\begin{array}{l}\oplus \oplus \Theta \Theta \\
\text { low }^{12}\end{array}$ & $\begin{array}{l}\text { CBT may reduce } \\
\text { anxiety when } \\
\text { compared with } \\
\text { other treatments. }\end{array}$ \\
\hline $\begin{array}{l}\text { Health-relat- } \\
\text { ed quality of } \\
\text { life at end of } \\
\text { treatment } \\
\text { Assessed } \\
\text { with: QoLI } \\
\text { Scale from: -6 } \\
\text { to } 6\end{array}$ & $\begin{array}{l}\text { By the end of } \\
\text { treatment, the } \\
\text { mean quality } \\
\text { of life score } \\
\text { increased from } \\
\text { a mean of } 1.98 \\
\text { (SD 1.58) to } \\
2.27(1.5) \text {. }\end{array}$ & $\begin{array}{l}\text { By the } \\
\text { end of } \\
\text { treatment, } \\
\text { the qual- } \\
\text { ity of life } \\
\text { score had } \\
\text { increased } \\
\text { from a } \\
\text { mean of } \\
1.67 \text { (SD } \\
1.71 \text { ) to } \\
2.32 \text { (SD } \\
1.51) \text {. }\end{array}$ & - & $\begin{array}{l}95 \\
(1 \mathrm{RCT})\end{array}$ & $\begin{array}{l}\oplus \Theta \Theta \Theta \\
\text { very } \\
\text { low }^{1,4,5}\end{array}$ & $\begin{array}{l}\text { We are uncertain } \\
\text { whether CBT im- } \\
\text { proves health-re- } \\
\text { lated quality of life } \\
\text { compared with } \\
\text { other treatments. }\end{array}$ \\
\hline $\begin{array}{l}\text { Negatively } \\
\text { biased inter- } \\
\text { pretations of } \\
\text { tinnitus at end } \\
\text { of treatment } \\
\text { Assessed } \\
\text { with: TAQ, } \\
\text { TCS, TCQ }\end{array}$ & - & $\begin{array}{l}\text { SMD } 0.55 \\
\text { lower } \\
\text { ( } 0.75 \text { lower } \\
\text { to } 0.35 \\
\text { lower) }\end{array}$ & - & $\begin{array}{l}455 \\
\text { (5 RCTs) }\end{array}$ & $\begin{array}{l}\oplus \oplus \oplus \ominus \\
\text { moderate }^{1}\end{array}$ & $\begin{array}{l}\text { CBT probably re- } \\
\text { duces negatively } \\
\text { biased interpre- } \\
\text { tations of tinnitus } \\
\text { when compared } \\
\text { with other treat- } \\
\text { ments. }\end{array}$ \\
\hline $\begin{array}{l}\text { Other adverse } \\
\text { effects }\end{array}$ & $\begin{array}{l}\text { No other advers } \\
\text { reported. }\end{array}$ & se effects & - & $\begin{array}{l}595 \\
\text { (6 RCTs) }\end{array}$ & $\begin{array}{l}\oplus \oplus \Theta \Theta \\
\text { low }^{13}\end{array}$ & - \\
\hline $\begin{array}{l}\text { Other adverse } \\
\text { effects }\end{array}$ & $\begin{array}{l}\text { No other advers } \\
\text { reported. }\end{array}$ & se effects & - & $\begin{array}{l}595 \\
\text { (6 RCTs) }\end{array}$ & $\begin{array}{l}\oplus \oplus \Theta \Theta \\
\text { low }^{13}\end{array}$ & - \\
\hline
\end{tabular}


${ }^{*}$ The risk in the intervention group (and its 95\% confidence interval) is based on the assumed risk in the comparison group and the relative effect of the intervention (and its $95 \% \mathrm{Cl}$ ).

BDI: Beck Depression Inventory; CI: confidence interval; DASS: Depression Anxiety and Stress Scale; GAD-7: Generalized Anxiety Disorder-7; HADS-A: Hospital Anxiety and Depression Scale-Anxiety; HADS-D: Hospital Anxiety and Depression Scale-Depression; HRSD: Hamilton Rating Scale for Depression; PHQ-9: Patient Health Questionnaire; QoLI: Quality of Life Inventory; RR: risk ratio; STAI: State Trait Anxiety Inventory; TAQ: Tinnitus Acceptance Questionnaire; TCQ: Tinnitus Cognitions Questionnaire; TCS: Tinnitus Catastrophizing Scale; TEQ-ED: Tinnitus Effects Questionnaire-Emotional Distress; TFI: Tinnitus Functional Inventory; THI: Tinnitus Handicap Inventory; THQ: Tinnitus Handicap Questionnaire; TQ: Tinnitus Questionnaire; TRQ: Tinnitus Reaction Questionnaire

\section{GRADE Working Group grades of evidence}

High certainty: We are very confident that the true effect lies close to that of the estimate of the effect

Moderate certainty: We are moderately confident in the effect estimate: The true effect is likely to be close to the estimate of the effect, but there is a possibility that it is substantially different

Low certainty: Our confidence in the effect estimate is limited: The true effect may be substantially different from the estimate of the effect

Very low certainty: We have very little confidence in the effect estimate: The true effect is likely to be substantially different from the estimate of effect

\section{Footnotes}

'Downgraded one level due to study limitations (risk of bias): all studies included for this outcome were judged to be either unclear or at high risk of performance bias due to an absence of blinding of participants and personnel.

${ }^{2}$ Downgraded one level due to inconsistency: not all the confidence intervals overlap, and statistical heterogeneity is relatively high and statistically significant.

${ }^{3}$ Downgraded one level due to study limitations (risk of bias): one study judged to be at high risk and all others, except one, at unclear risk of attrition bias.

${ }^{4}$ Downgraded one level due to imprecision: overall confidence interval crosses the line of no effect.

${ }^{5}$ Downgraded one level due to imprecision: small sample size. 


\section{References to studies}

\section{Included studies}

Abbott 2009

[DOI: 10.1080/16506070902763174]

Abbott JA, Kaldo V, Klein B, Austin D, Hamilton C, Piterman L, et al. A cluster randomised trial of an internet-based intervention program for tinnitus distress in an industrial setting. Cognitive Behaviour Therapy 2009;38(3):162-73. [DOI: 10.1080/16506070902763174]

Andersson 2002

* Andersson G, Stromgren T, Strom L, Lyttkens L. Randomized controlled trial of internetbased cognitive behavior therapy for distress associated with tinnitus. Psychosomatic Medicine 2002;64(5):810-6. [DOI: 10.1097/01.PSY.0000031577.42041.F8]

Andersson 2005

* Andersson G, Porsaeus D, Wiklund M, Kaldo V, Larsen HC. Treatment of tinnitus in the elderly: a controlled trial of cognitive behavior therapy. International Journal of Audiology 2005;44(11):671-5. [DOI: 10.1080/14992020500266720]

Arif 2017

Arif M, Sadlier M, Rajenderkumar D, James J, Tahir T. A randomised controlled study of mindfulness meditation versus relaxation therapy in the management of tinnitus. Journal of Laryngology and Otology 2017;131(6):501-7. [DOI: 10.1017/ S002221511700069X]

Beukes 2018a

Published and unpublished data [ClinicalTrials.gov: NCT02370810]

* Beukes EW, Baguley DM, Allen PM, Manchaiah V, Andersson G. Audiologist- guided internet-based cognitive behavior therapy for adults with tinnitus in the United Kingdom: a randomized controlled trial. Ear and Hearing 2018;39(3):423-33. [Other: 0196-0202]

NCT02370810. A CBT-based internet intervention for adults with tinnitus in the United Kingdom [Study protocol for a CBT-based internet intervention for adults with tinnitus in the United Kingdom: a randomised controlled trial]. clinicaltrials.gov/ show/NCT02370810 (first received 25 February 2015). [CENTRAL: CN-01050683; CRS: 1781268; CRSREP: 1781268]

Beukes 2018b

[ClinicalTrials.gov: NCT02665975]

* Beukes EW, Andersson G, Allen PM, Manchaiah V, Baguley D. Guided internet-based versus face-to-face clinical care in the treatment of tinnitus: a multicentre randomized non-inferiority trial. JAMA Otolaryngology-Head \& Neck Surgery 2018;144(12):112633. [DOI: 10.1001/jamaoto.2018.2238] 
NCT02665975. Internet-based versus face-to-face clinical care for tinnitus [Internetbased versus face-to-face clinical care for tinnitus: a multi-study randomised control trial]. clinicaltrials.gov/show/NCT02665975 (first received 28 January 2016). [CENTRAL: CN-01180255; CRS: 4390326; CRSREP: 4390326]

Cima 2012

Published and unpublished data [ClinicalTrials.gov: NCT00733044]

* Cima RF, Maes IH, Joore MA, Scheyen DJ, El Refaie A, Baguley DM, et al. Specialised treatment based on cognitive behaviour therapy versus usual care for tinnitus: a randomised controlled trial. Lancet 2012;379:1951-9.

NCT00733044. Cost-effectiveness of multidisciplinary management of tinnitus [Costeffectiveness of multidisciplinary management of tinnitus at a specialised tinnitus centre]. clinicaltrials.gov/show/NCT00733044 (first received 12 August 2008). [CENTRAL: CN-00874706; CRS: 1648542; CRSREP: 1648542]

Davies 1995

[DOI: 10.1080/08870449508401943]

Davies S, McKenna L, Hallam RS. Relaxation and cognitive therapy: a controlled trial in chronic tinnitus. Psychology \& Health 1995;10(2):129-43.

Henry 1996

* Henry JL, Wilson PH. The psychological management of tinnitus: comparison of a combined cognitive educational program, education alone and a waiting-list control. International Tinnitus Journal 1996;2:9-20. [PubMed: 10753339]

Henry 1998

Published data only (unpublished sought but not used)

* Henry JL, Wilson PH. An evaluation of two types of cognitive intervention in the management of chronic tinnitus. Scandinavian Journal of Behaviour Therapy 1998;27(4):156-66. [DOI: 10.1080/02845719808408510]

Hesser 2012

Hesser H, Gustafsson T, Lunden C, Henrikson O, Fattahi K, Johnsson E, et al. A randomized controlled trial of internet-delivered cognitive behavior therapy and acceptance and commitment therapy in the treatment of tinnitus. Journal of Consulting and Clinical Psychology 2012;80(4):649-61. [DOI: 10.1037/a0027021]

Jakes 1986

* Jakes S, Hallam R, Rachman S, Hinchcliffe R. The effects of reassurance relaxation training and distraction on chronic tinnitus sufferers. Behavior Research and Therapy 1986;24(5):497-507. [DOI: 10.1016/0005-7967(86)90029-X]

Jakes 1992

* Jakes SC, Hallam RS, McKenna L, Hinchcliffe R. Group cognitive therapy for medical patients - an application to tinnitus. Cognitive Therapy and Research 1992;16(1):6782. [DOI: 10.1007/BF01172957]

Jasper 2014

[ClinicalTrials.gov: NCT01205906] 
* Jasper K, Weise C, Conrad I, Andersson G, Hiller W, Kleinstauber M. Internet-based guided self-help versus group cognitive behavioral therapy for chronic tinnitus: a randomized controlled trial. Psychotherapy and Psychosomatics 2014;83(4):234-46.

NCT01205906. Comparison of an internet-based guided self-help and a group therapy for chronic tinnitus [Comparison of the efficacy of an internet-based self-help training and a well-established outpatient group therapy for the treatment of chronic tinnitus: a randomized controlled trial]. clinicaltrials.gov/show/nct01205906 (first received 21 September 2010). [CENTRAL: CN-00921448; CRS: 1689266; CRSREP: 1689266]

Kaldo 2007

Kaldo V, Cars S, Rahnert M, Larsen HC, Andersson G. Use of a self-help book with weekly therapist contact to reduce tinnitus distress: a randomized controlled trial. Journal of Psychosomatic Research 2007;63(2):195-202.

Kreuzer 2012

[ClinicalTrials.gov: NCT01540357]

Kreuzer PM, Goetz M, Holl M, Schecklmann M, Landgrebe M, Staudinger S, et al. Mindfulness-and body-psychotherapy-based group treatment of chronic tinnitus: a randomized controlled pilot study. BMC Complementary and Alternative Medicine 2012;12:235.

NCT01540357. Mindfulness-based therapy in chronic tinnitus [Mindfulness-based therapy for the treatment of chronic tinnitus: a randomized controlled pilot study]. clinicaltrials.gov/show/NCT01540357 (first received 28 February 2012). [CENTRAL: CN-00921453; CRS: 1689271; CRSREP: 1689271]

Lindberg 1989

Published data only (unpublished sought but not used)

Lindberg P, Scott B, Melin L, Lyttkens L. The psychological treatment of tinnitus: an experimental evaluation. Behaviour Research and Therapy 1989;27(6):593-603. [DOI: 10.1016/0005-7967(89)90143-5]

Malinvaud 2016

Published data only (unpublished sought but not used)

Malinvaud D, Londero A, Niarra R, Peignard P, Warusfel O, Viaud-Delmon I, et al. Auditory and visual $3 \mathrm{D}$ virtual reality therapy as a new treatment for chronic subjective tinnitus: results of a randomized controlled trial. Hearing Research 2016;333:127-35. [DOI: 10.1016/j.heares.2015.12.023]

Martz 2018

[ClinicalTrials.gov: 02293512]

Martz E, Chesney M, Livneh H, Jelleberg C, Fuller B, Henry JA. A pilot randomized clinical trial comparing three brief group interventions for individuals with tinnitus. Global Advances in Health and Medicine 2018;7:1-11. 
NCT02293512. A comparison of three psychoeducational group interventions for veterans with tinnitus [A comparison of $\mathrm{CBT}$ and CET interventions for veterans with tinnitus]. clinicaltrials.gov/show/NCT02293512 (first received 18 November 2014). [CENTRAL: CN-01039743; CRS: 1770339; CRSREP: 1770339]

\section{McKenna 2017}

[ClinicalTrials.gov: NCT02059447]

McKenna L, Marks EM, Hallsworth CA, Schaette R. Mindfulness-based cognitive therapy as a treatment for chronic tinnitus: a randomized controlled trial. Psychotherapy and Psychosomatics 2017;86(6):351-61. [Other: 0033-3190]

NCT02059447. A comparison of the benefits of mindfulness based cognitive therapy, relaxation therapy and a waiting list control in the management of distress in chronic tinnitus patients. clinicaltrials.gov/show/nct02059447 (first received 11 February 2014). [CENTRAL: CN-00994037; CRS: 1724717; CRSREP: 1724717]

Nyenhuis 2013a

Nyenhuis N, Zastrutzki S, Weise C, Jager B, Kroner-Herwig B. The efficacy of minimal contact interventions for acute tinnitus: a randomised controlled study. Cognitive Behaviour Therapy 2013;42(2):127-38. [DOI: 10.1080/16506073.2012.655305]

Oron (unpublished)

Unpublished data only [ClinicalTrials.gov: NCT03068871]

Martz E, Chesney MA, Livneh H, Ungar OJ, Harel S, Terracini D, Oron Y. Two brief group interventions for individuals with tinnitus in Israel. Unpublished.

NCT03068871. A comparison of two psycho-educational group interventions for tinnitus patients. https://clinicaltrials.gov/show/nct03068871 (first received 3 March 2017). [CENTRAL: CN-01343644; CRS: 5479734; CRSREP: 5479734]

Philippot 2012a

Philippot P, Nef F, Clauw L, Romree M, Segal Z. A randomized controlled trial of mindfulness-based cognitive therapy for treating tinnitus. Clinical Psychology \& Psychotherapy 2012;19(5):411-9. [Other: 10.1002/cpp.756]

Robinson 2008

Robinson SK, Viirre ES, Bailey KA, Kindermann S, Minassian AL, Goldin PR, et al. A randomized controlled trial of cognitive-behavior therapy for tinnitus. International Tinnitus Journal 2008;14(2):119-26.

Schmidt 2018

Published and unpublished data [ClinicalTrials.gov: NCT00724152]

NCT00724152. Cognitive behavioral therapy (CBT) for tinnitus. clinicaltrials.gov/show/ NCT00724152 (first received 29 July 2008). [CENTRAL: CN-00921437; CRS: 1689255; CRSREP: 1689255]

* Schmidt CJ, Kerns RD, Finkel S, Michaelides E, Henry JA. Cognitive-behavioral therapy for veterans with tinnitus. Federal Practitioner 2018;35(8):36-46. [PubMed: 30766380] 
Chapter 3

Weise 2016

[ClinicalTrials.gov: NCT01205919]

NCT01205919. Internet-based guided self-help for chronic tinnitus [Efficacy of an internet-based guided self-help training for chronic tinnitus: a randomized controlled trial]. clinicaltrials.gov/show/NCT01205919 (first received 21 September 2010). [CENTRAL: CN-00921442; CRS: 1689260; CRSREP: 1689260]

* Weise C, Kleinstauber M, Andersson G. Internet-delivered cognitive-behavior therapy for tinnitus: a randomized controlled trial. Psychosomatic medicine 2016;78(4):501-10.

Westin 2011

Westin VZ, Schulin M, Hesser H, Karlsson M, Noe RZ, Olofsson U, et al. Acceptance and commitment therapy versus tinnitus retraining therapy in the treatment of tinnitus: a randomised controlled trial. Behaviour Research and Therapy 2011;49(11):737-47. [DOI: 10.1016/j.brat.2011.08.001]

Zhong 2014

Published data only (unpublished sought but not used)

* Zhong C, Zhong Z, Luo Q, Qiu Y, Yang Q, Liu Y. The curative effect of cognitive behavior therapy for the treatment of chronic subjective tinnitus. Lin Chuang $\mathrm{Er} \mathrm{Bi}$ Yan Hou Tou Jing Wai Ke za Zhi [Journal of Clinical Otorhinolaryngology, Head, and Neck Surgery] 2014;29(8):709-11. [PubMed: 26248442]

\section{Excluded studies}

Delb 2002

Delb W, D'Amelio R, Boisten CJ, Plinkert PK. Evaluation of the tinnitus retraining therapy as combined with a cognitive behavioral group therapy. HNO 2002;50(11):997-1004.

Henry 2017

Henry JA, Thielman EJ, Zaugg TL, Kaelin C, Schmidt CJ, Griest S, et al. Randomized controlled trial in clinical settings to evaluate effectiveness of coping skills education used with progressive tinnitus management. Journal of Speech, Language, and Hearing Research 2017;60(5):1378-97.

Kaldo 2008

* Kaldo V, Levin S, Widarsson J, Buhrman M, Larsen HC, Andersson G. Internet versus group cognitive-behavioral treatment of distress associated with tinnitus: a randomized controlled trial. Behavior Therapy 2008;39(4):348-59.

Konzag 2006

Konzag TA, Rubler D, Bloching M, Bandemer-Greulich U, Fikentscher E, Frommer J. Counselling versus a self-help manual for tinnitus outpatients: a comparison of effectiveness. HNO 2006;54(8):599-604.

Kröner-Herwig 1995

Kröner-Herwig B, Hebing G, van Rijn-Kalkmann U, Frenzel A, Schilkowsky G, Esser G. The management of chronic tinnitus--comparison of a cognitive-behavioural group training with yoga. Journal of Psychosomatic Research 1995;39(2):153-65. 
Kröner-Herwig 1999

Kroener-Herwig B, Esser G, Frenzel A, Fritsche G, Schilkowsky G. Result of an outpatient cognitive-behavioral group treatment for chronic tinnitus. In: Proceedings of the Sixth International Tinnitus Seminar, Cambridge, UK, 5-9 September, 1999. 1999:369-72.

Kröner-Herwig 2003

Kroner-Herwig B, Frenzel A, Fritsche G, Schilkowsky G, Esser G. The management of chronic tinnitus: comparison of an outpatient cognitive-behavioral group training to minimal-contact interventions. Journal of Psychosomatic Research 2003;54(4):381-9.

Kröner-Herwig 2006

Kroner-Herwig B, Zachriat C, Weigand D. Do patient characteristics predict outcome in the outpatient treatment of chronic tinnitus? Psycho-social Medicine 2006;3:Doc07.

Li 2019

Juan L, Jianhua J, Songli X, Qian Z, Yuqin C, Min H, et al. Clinical efficacy of cognitive behavioral therapy for chronic subjective tinnitus. American Journal of Otolaryngology 2019;40:253-6.

Luyten 2019

Luyten T, Van de Heyning P, Jacquemin L, Van Looveren N, Declau F, Fransen E, et al. The value of Eye Movement Desensitization Reprocessing in the treatment of tinnitus: study protocol for a randomized controlled trial. Trials 2019;20:32.

Malouff 2010

Malouff JM, Noble W, Schutte NS, Bhullar N. The effectiveness of bibliotherapy in alleviating tinnitus-related distress. Journal of Psychosomatic Research 2010;68(3):245-51.

Rief 2005

Rief W, Weise C, Kley N, Martin A. Psychophysiologic treatment of chronic tinnitus: a randomized clinical trial. Psychosomatic Medicine 2005;67(5):833-8.

\section{Scott 1985}

Scott B, Lindberg P, Lyttkens L, Melin L. Psychological treatment of tinnitus. An experimental group study. Scandinavian Audiology 1985;14(4):223-30.

Tan 2018

Tan Y, Chen L, Luo Z, Ye W. The therapeutic effect of rehabilitation exercise and cognitive-behavioral intervention on the patients with tinnitus. Modern Clinical Nursing 2018;17(2):34-9.

Tavakoli 2019

Tavakoli M, Marashi S, Hamid N, Beshlideh K. The effects of cognitive-behavioral training and muscle relaxation on the degree of tinnitus and the quality of sleep. Auditory and Vestibular Research 2019;28(3):182-9. 


\section{Tucker 2013}

Tucker EM. Tinnitus in Cochlear Implantees: Cognitive Behavioural Therapy for Cochlear Implant Users [Doctoral Thesis]. Available from: https://eprints.soton.ac.uk/. University of Southampton, Faculty of Engineering and the Environment, 2013.

Weise 2008

Weise C, Heinecke K, Rief W. Biofeedback-based behavioral treatment for chronic tinnitus: results of a randomized controlled trial. Journal of Consulting and Clinical Psychology 2008;76(6):1046-57.

Zachriat 2004

Zachriat C, Kroner-Herwig B. Treating chronic tinnitus: comparison of cognitivebehavioural and habituation-based treatments. Cognitive Behaviour Therapy 2004;33(4):187-98.

Zenner 2013

[ClinicalTrials.gov: NCT 00719940]

NCT00719940. Tinnitus treatment by structured cognitive behavioral therapy [Randomized controlled clinical trial of efficacy and safety of individual cognitive behavioral therapy (CBT) within the setting of the structured therapy program sTCP (structured tinnitus care program) in patients with tinnitus aurium]. clinicaltrials.gov/ show/nct00719940 (first received 22 July 2008). [CENTRAL: CN-00725092; CRS: 1526940; CRSREP: 1526940]

Zenner HP, Vonthein R, Zenner B, Leuchtweis R, Plontke SK, Torka W, et al. Standardized tinnitus-specific individual cognitive-behavioral therapy: a controlled outcome study with 286 tinnitus patients. Hearing Research 2013;298:117-25.

Zhicheng 2019

[Other: ChiCTR1900023179]

Zhicheng L. Resilience training to improve tinnitus distress: a randomized controlled trial. International Clinical Trials Registry 2019.

\section{Ongoing studies}

NCT03022084

[ClinicalTrials.gov: NCT03022084]

NCT03022084. Clinical trial of sound-based versus behavioral therapy for tinnitus. clinicaltrials.gov/show/NCT03022084 (first received 16 January 2017).

NCT03386123

[ClinicalTrials.gov: NCT03386123]

NCT03386123. A comparison of CBTi and usual treatment for tinnitus related insomnia [A comparison of cognitive behaviour therapy for insomnia (CBTi) and usual audiological rehabilitation in the management of tinnitus related insomnia]. clinicaltrials.gov/show/NCT03386123 (first received 29 December 2017). 
NCT04004260

[ClinicalTrials.gov: NCT04004260]

Manchaiah V. CBT-based internet intervention for adults with tinnitus in the United States. https://clinicaltrials.gov/ct2/show/nct04004260 (first received 2 July 2019).

\section{NTR6415}

[Other: NTR6415]

NTR6415. Bold exposure or safe masking? A fear-conditioning approach to chronic tinnitus suffering [Bold exposure or safe masking? A fear-conditioning approach to chronic tinnitus suffering; a randomised controlled trial]. trialregister.nl/trial/6235 (first received 24 April 2017).

SLCTR/2018/005

[Other: SLCTR/2018/005]

SLCTR/2018/005. Effectiveness of cognitive behavioral therapy for tinnitus [Effectiveness of modified cognitive behavioral therapy (CBT) in reducing distress for patients with tinnitus who are receiving treatment at two tertiary care hospitals in Sri Lanka, a randomised controlled trial]. slctr.lk/trials/slctr-2018-005 (first received 2 February 2018).

\section{Other references}

\section{Additional references}

Andersson 1999

Andersson G, Lyttkens L. A meta-analytic review of psychological treatments for tinnitus. British Journal of Audiology 1999;33(4):201-10. [PubMed: 10509855]

Andersson 2001

Andersson G. The role of psychology in managing tinnitus: a cognitive behavioural approach. Seminars in Hearing 2001;22:65-76.

Andersson 2004

Andersson G, Kaldo V. Internet-based cognitive behavioral therapy for tinnitus. Journal of Clinical Psychology 2004;60(2):171-8. [0021-9762: (Print)]

Andersson 2009

Andersson G. Tinnitus patients with cognitive problems: causes and possible treatments. The Hearing Journal 2009;62:27-8,30.

Baguley 2013

Baguley D, Andersson G, McFerran D, McKenna L. Tinnitus: A Multidisciplinary Approach. Second edition. Chichester: John Wiley \& Sons, Ltd, 2013.

Bakal 1972

Bakal D. The Psychobiology of Chronic Headache. New York: Springer, 1972.

Beck 1979

Beck AT. Cognitive Therapy and the Emotional Disorders. New York: International Universities Press, 1979. 
Chapter 3

\section{Beck 1988}

Beck AT, Epstein N, Brown G, Steer RA. An inventory for measuring clinical anxiety: psychometric properties. Journal of Consulting and Clinical Psychology 1988;56(6):893-7.

\section{Beck 1996}

Beck AT, Steer RA, Brown GK. Manual for the Beck Depression Inventory-II. San Antonio, TX: Psychological Corporation, 1996.

\section{Bernstein 1973}

Bernstein DA, Borkovec TD. Progressive Relaxation Training: A Manual for the Helping Professions. Champaign, IL, US: Research Press, 1973.

\section{Bernstein 1984}

Bernstein DA, Borkovec TD. Progressive Relaxation Training. Champaign, Illinois: Research Press, 1984.

\section{Beukes 2018c}

Beukes E, Allen P, Baguley D, Manchaiah V, Andersson G. Long-term efficacy of audiologist-guided internet-based cognitive behavior therapy for tinnitus. American Journal of Audiology 2018;27:431-47. [DOI: 10.1044/2018_AJA-IMIA3-18-0004]

\section{Beukes 2019}

Beukes E, Manchaiah V, Allen P, Baguley D, Andersson G. Internet-based interventions for adults with hearing loss, tinnitus and vestibular disorders: A systematic review and meta-analysis. Trends in Hearing 2019;23:1-22.

Biesinger 1998

Biesinger E, Heiden C, Greimel V, Lendle T, Höing R, Albegger K. Strategien in der ambulaten Behandlung des Tinnitus. HNO 1998;46(2):157-69. [Other: 0017-6192]

\section{Bishop 2004}

Bishop SR, Lau M, Shapiro S, Carlson L, Anderson ND, Carmody J, et al. Mindfulness: a proposed operational definition. Clinical Psychology: Science and Practice 2004;11(3):230-41.

\section{Bouton 2007}

Bouton M. Learning and Behavior: A Contemporary Synthesis. Sunderland: MA Sinauer, 2007.

\section{Buss 1998}

Buss E, Hall III JW, Grose JH, Hatch DR. Perceptual consequences of peripheral hearing loss: do edge effects exist for abrupt cochlear lesions? Hearing Research 1998;125:98-108.

\section{Carver 1989}

Carver CS, Scheier MF, Weintraub JK. Assessing coping strategies: a theoretically based approach. Journal of Personality and Social Psychology 1989;56(2):267-83.

\section{Carver 1997}

Carver CS. You want to measure coping but your protocol's too long: consider the brief COPE. International Journal of Behavioral Medicine 1997;4(1):92-100. 
Chan 2013

Chan A-W, Tetzlaff JM, Altman DG, Laupacis A, Gøtzsche PC, Krleža-Jerić K, et al. SPIRIT 2013 Statement: defining standard protocol items for clinical trials. Annals of Internal Medicine 2013;158(3):200-7.

Cima 2011a

Cima RF, Vlaeyen JW, Maes IH, Joore MA, Anteunis LJ. Tinnitus interferes with daily life activities: a psychometric examination of the Tinnitus Disability Index. Ear and Hearing 2011;32(5):623-33. [PubMed: 21336139]

Cima 2011b

Cima RF, Crombez G, Vlaeyen JW. Catastrophizing and fear of tinnitus predict quality of life in patients with chronic tinnitus. Ear and Hearing 2011;32(5):634-64.

Cima 2014

Cima RFF, Andersson G, Schmidt CJ, Henry JA. Cognitive-behavioral treatments for tinnitus: a review of the literature. Journal of the American Academy of Audiology 2014;25:29-61.

Cima 2019

Cima RFF, Mazurek B, Haider H, Kikidis D, Lapira A, Norena A, et al. A multidisciplinary European guideline for tinnitus: diagnostics, assessment, and treatment. HNO 2019;67(Suppl 1):10-42.

Crönlein 2007

Crönlein T, Langguth B, Geisler P, Hajak G. Tinnitus and insomnia. Progress in Brain Research 2007;166:227-33.

Davis 2000

Davis A, El Refaie A. Epidemiology of tinnitus. In: Tyler RS, editor(s). Tinnitus Handbook. Singular, Thomson Learning, 2000.

de Ridder 2011

De Ridder D, Elgoyhen AB, Romo R, Langguth B. Phantom percepts: tinnitus and pain as persisting aversive memory networks. Proceedings of the National Academy of Sciences of the United States of America 2011;108(20):8075-80.

de Ridder 2014

De Ridder D, Vanneste S, Weisz N, Londero A, Schlee W, Elgoyhen AB, et al. An integrative model of auditory phantom perception: tinnitus as a unified percept of interacting separable subnetworks. Neuroscience and Biobehavioral Reviews 2014;44:16-32.

Department of Health 2009

Department of Health. Provision of Services for Adults with Tinnitus. A Good Practice Guide. London: Central Office of Information, 2009.

Diener 1985

Diener E, Emmons RA, Larsen RJ, Griffin S. The Satisfaction With Life Scale. Journal of Personality Assessment 1985;49:71-5. 
Chapter 3

Dietrich 2001

Dietrich V, Nieschalk M, Stoll W, Rajan R, Pantev C. Cortical reorganization in patients with high frequency cochlear hearing loss. Hearing Research 2001;158:95-101.

Dobie 1999

Dobie RA. A review of randomized clinical trials in tinnitus. Laryngoscope 1999;109:1202-11.

Dong 2010

Dong S, Rodger J, Mulders WH, Robertson D. Tonotopic changes in GABA receptor expression in guinea pig inferior colliculus after partial unilateral hearing loss. Brain Research 2010;1342:24-32.

Ecclestone 2013

Eccleston C, Morley SJ, Williams AC. Psychological approaches to chronic pain management: evidence and challenges. British Journal of Anaesthesia 2013;111(1):59-63.

Eggermont 2004

Eggermont JJ, Roberts LE. The neuroscience of tinnitus. Trends in Neuroscience 2004;27:676-82.

Eggermont 2014

Eggermont JJ, Roberts LE. Tinnitus: animal models and findings in humans. Cell and Tissue Research 2014;361(1):311-36.

El-Shunnar 2011

El-Shunnar S, Hoare DJ, Smith S, Gander PE, Kang S, Fackrell K, et al. Primary care for tinnitus: practice and opinion among GPs in England. Journal of Evaluation in Clinical Practice 2011;17:684-92.

Elgoyhen 2015

Elgoyhen AB, Langguth B, De Ridder D, Vanneste S. Tinnitus: perspectives from human neuroimaging. Nature Reviews. Neuroscience 2015;16(10):632-42.

Ellis 1977

Ellis A, Grieger R (editors). Handbook of Rational-Emotive Therapy. Vol. 1. New York: Springer, 1977.

Engineer 2011

Engineer ND, Riley JR, Seale JD, Vrana WA, Shetake JA, Sudanagunta SP, et al. Reversing pathological neural activity using targeted plasticity. Nature 2011;470:101-4.

Fackrell 2014

Fackrell K, Hall DA, Barry J, Hoare DJ. Tools for tinnitus measurement: development and validity of questionnaires to assess handicap and treatment effects. In: Signorelli F, Turjman F, editor(s). Tinnitus: Causes, Treatment and Short \& Long-Term Health Effects. New York: Nova Science Publisher, 2014:13-60.

Fowler 1944

Fowler EP. Head noises in normal and disordered ears: significance, measurement, differentiation and treatment. Archives of Otolaryngology 1944;39:490-503. 


\section{Frisch 1992}

Frisch MB, Cornell J, Villanueva M, \& PJ. Clinical validation of the Quality of Life Inventory: a measure of life satisfaction of use in treatment planning and outcome assessment. Psychological Assessment 1992;4:92-101.

Fuller 2017a

Fuller TE, Haider HF, Kikidis D, Lapira A, Mazurek B, Norena A, et al. Different teams, same conclusions? A systematic review of existing clinical guidelines for the assessment and treatment of tinnitus in adults. Frontiers in Psychology 2017;8:206. [DOI: 10.3389/fpsyg.2017.00206]

Furlong 2001

Furlong WJ, Feeny DH, Torrance GW, Barr RD. The Health Utilities Index (HUI®) system for assessing health-related quality of life in clinical studies. Annals of Medicine 2001;33:375-84.

Gallus 2015

Gallus S, Lugo A, Garavello W, Bosetti C, Santoro E, Colombo P, et al. Prevalence and determinants of tinnitus in the Italian adult population. Neuroepidemiology 2015;45(1):12-9.

Goebel 1994

Goebel G, Hiller W. The Tinnitus Questionnaire. A standard instrument for grading the degree of tinnitus. Results of a multicenter study with the tinnitus questionnaire [Tinnitus-Fragebogen (TF). Standardinstrument zur Graduierung des Tinnitusschweregrades. Ergebnisse einer Multicenterstudie mit dem TinnitusFragebogen (TF)]. HNO 1994;42(3):166-72.

Hahn 2008

Hahn A, Radkova R, Achiemere G, Klement V, Alpini D, Strouhal J. Multimodal therapy for chronic tinnitus. International Tinnitus Journal 2008;14:69-71.

Hall 2011

Hall D, Láinez M, Newman C, Sanchez T, Egler M, Tennigkeit F, et al. Treatment options for subjective tinnitus: self reports from a sample of general practitioners and ENT physicians within Europe and the USA. BMC Health Services Research 2011;11(1):302.

Hall 2016

Hall D, Haider H, Szczepek A, Lau P, Rabau S, Jones-Diette J, et al. Systematic review of outcome domains and instruments used in clinical trials of tinnitus treatments in adults. Trials 2016;17(1):270. [DOI: 10.1186/s13063-016-1399-9]

Hall 2018

Hall DA, Smith H, Hibbert A, Colley V, Haider HF, Horobin A, et al; Core Outcome Measures in Tinnitus (COMiT) initiative. The COMiT'ID Study: developing core outcome domains sets for clinical trials of sound-, psychology-, and pharmacologybased interventions for chronic subjective tinnitus in adults. Trends in Hearing 2018;22:1-16. 
Hallam 1984

Hallam RS, Rachman S, Hinchcliffe R. Psychological aspects of tinnitus. In: Rachman S, editor(s). Contributions to Medical Psychology. Oxford: Pergamon Press, 1984.

Hallam 1988

Hallam RS, Jakes SC, Hinchcliffe R. Cognitive variables in tinnitus annoyance. British Journal of Clinical Psychology 1988;27:213-22.

Hallam 2008

Hallam RS. Manual of the Tinnitus Questionnaire (TQ). Revised and updated. London: Polpresa Press, 2008.

Hamilton 1960

Hamilton M. A rating scale for depression. Journal of Neurology, Neurosurgery \& Psychiatry 1960;23(1):56-62.

Han 2009

Han BI, Lee HW, Kim TY, Lim JS, Shin KS. Tinnitus: characteristics, causes, mechanisms, and treatments. Journal of Clinical Neurology 2009;5:11-9.

Hayes 1999

Hayes SC, Strosahl KD, Wilson KG. Acceptance and Commitment Therapy: An Experiential Approach to Behavior Change. New York: Guildford Press, 1999.

Hays 1993

Hays RD, Sherbourne CD, Mazel RM. The RAND 36-item health survey 1.0. Health Economics 1993;2(3):217-27.

Henry 1996a

Henry JL, Wilson PH. The psychological management of tinnitus: comparison of a combined cognitive educational program, education alone and a waiting-list control. International Tinnitus Journal 1996;2:9-20.

Henry 1998a

Henry JL, Wilson P. The psychometric properties of two measures of tinnitus complaint and handicap. International Tinnitus Journal 1998;4:114-21.

Henry 2005

Henry JA, Dennis KC, Schechter MA. General review of tinnitus: prevalence, mechanisms, effects, and management. Journal of Speech, Language, and Hearing Research 2005;48:1204-35.

Hesser 2009

Hesser H, Westin V, Hayes SC, Andersson G. Clients' in-session acceptance and cognitive defusion behaviors in acceptance-based treatment of tinnitus distress. Behaviour Research and Therapy 2009;47(6):523-8. [PubMed: 19268281]

Hesser 2011

Hesser H, Weise C, Westin VZ, Andersson G. A systematic review and meta-analysis of randomized controlled trials of cognitive-behavioral therapy for tinnitus distress. Clinical Psychology Review 2011;31(4):545-53. 
Higgins 2011

Higgins JPT, Green S (editors). Cochrane Handbook for Systematic Reviews of Interventions Version 5.1.0 [updated March 2011]. The Cochrane Collaboration, 2011. Available from www.cochrane-handbook.org.

Higgins 2013

Higgins JPT, Lasserson T, Chandler J, Tovey D, Churchill R. Standards for the conduct of new Cochrane Intervention Reviews 2012 V2.3. Methodological Expectations of Cochrane Intervention Reviews: Standards for the conduct and reporting of new Cochrane Intervention Reviews 2012. Version 2. London: Cochrane, 2013.

Higgins 2016

Higgins JPT, Lasserson T, Chandler J, Tovey D, Churchill R. Methodological Expectations of Cochrane Intervention Reviews. London: Cochrane, 2016.

Hiller 2006

Hiller W, Goebel G. Factors influencing tinnitus loudness and annoyance. Archives of Otolaryngology--Head and Neck Surgery 2006;132:1323-30.

Hoare 2011a

Hoare DJ, Hall DA. Clinical guidelines and practice: a commentary on the complexity of tinnitus management. Evaluation and the Health Professions 2011;34(4):413-20.

Hoare 2011b

Hoare DJ, Kowalkowski V, Kang S, Hall DA. Systematic review and meta-analyses of RCTs examining tinnitus management. Laryngoscope 2011;121:1555-64.

Hoare 2014

Hoare DJ, Edmondson-Jones M, Sereda M, Akeroyd MA, Hall D. Amplification with hearing aids for patients with tinnitus and co-existing hearing loss. Cochrane Database of Systematic Reviews 2014, Issue 1. Art. No.: CD010151. DOI: 10.1002/14651858.CD010151.pub2.

Hobson 2012

Hobson J, Chisholm E, El Refaie A. Sound therapy (masking) in the management of tinnitus in adults. Cochrane Database of Systematic Reviews 2012, Issue 11. Art. No.: CD006371. DOI: 10.1002/14651858.CD006371.pub3.

Hofmann 2008

Hofmann SG, Asmundson G. Acceptance and mindfulness-based therapy: new wave or old hat? Clinical Psychology Review 2008;28(1):1-16.

Holl 2011

Holl M. Die Tinnitus-Atemtherapie: So gehen Sie aktiv gegen Ihr Ohrgeräusch vor. Hannover: Schluetersche, 2011.

Jacobson 1957

Jacobson E. You Must Relax. New York: McGraw-Hill, 1957. 
Jacobson 1991

Jacobson NS, Truax P. Clinical significance: a statistical approach to defining meaningful change in psychotherapy research. Journal of Consulting and Clinical Psychology 1991;59(1):12-9.

Jastreboff 1988

Jastreboff PJ, Brennan JF, Coleman JK, Sasaki CT. Phantom auditory sensation in rats: an animal model for tinnitus. Behavioral Neuroscience 1988;102:811-22.

Jastreboff 1990

Jastreboff PJ. Phantom auditory perception (tinnitus): mechanisms of generation and perception. Neuroscience Research 1990;8(4):221-54.

Jastreboff 1993

Jastreboff PJ, Hazell JW. A neurophysiological approach to tinnitus: clinical implications. British Journal of Audiology 1993;27(1):7-17. [PubMed: 8339063]

Jastreboff 2004

Jastreboff PJ, Hazell JWP. Tinnitus Retraining Therapy. Cambridge University Press, 2004.

Kabat-Zinn 1982

Kabat-Zinn J. An outpatient program in behavioral medicine for chronic pain patients based on the practice of mindfulness meditation: Theoretical considerations and preliminary results. General Hospital Psychiatry 1982;4(1):33-47.

Kaldo 2004a

Kaldo V, Andersson G. Kognitiv Beteendeterapivid Tinnitus [Cognitive-Behavioural Therapy of Tinnitus]. Lund, Sweden: Studentlitteratur, 2004.

Kaplan 1996

Kaplan R, Ganiats T, Sieber W. The Quality of Well-Being Scale, Self Administered (QWB-SA). San Diego: University of California, San Diego, 1996.

Kim 2015

Kim HJ, Lee HJ, An SY, Sim S, Park B, Kim SW, et al. Analysis of the prevalence and associated risk factors of tinnitus in adults. PloS One 2015;10(5):e0127578.

Kircher 2008

Kircher ML, Standring RT, Leonetti JP. Neuroaudiologic assessment of pulsatile tinnitus. In: Paper presented at the Annual Meeting of the American Academy of Otolaryngology - Head Neck Surgery. Chicago, 2008.

Kleinjung 2016

Kleinjung T. Pulsatile tinnitus. In: Baguley D, Fagelson M, editor(s). Tinnitus. Clinical and Research Perspectives. San Diego: Plural Publishing, 2016:163-80.

Kleinstauber 2013

Kleinstauber M, Jasper K, Schweda I, Hiller W, Andersson G, Weise C. The role of fear-avoidance cognitions and behaviors in patients with chronic tinnitus. Cognitive Behaviour Therapy 2013;42(2):84-99. [PubMed: 23199238] 
Kluk 2006

Kluk K, Moore BCJ. Dead regions in the cochlea and enhancement of frequency discrimination: effects of audiogram slope, unilateral versus bilateral loss, and hearing-aid use. Hearing Research 2006;221:1-15.

Kroenke 2001

Kroenke K, Spitzer RL, Williams JB. The PHQ-9: validity of a brief depression severity measure. Journal of General Internal Medicine 2001;16(1):606-13.

Kröner-Herwig 1997

Kröner-Herwig B. Psychologische Behandlung des Chronischen Tinnitus. Stuttgart: Beltz Psychologie Verlags Union, 1997.

Kuk 1990

Kuk FK, Tyler RS, Russell D, Jordan H. The psychometric properties of a tinnitus handicap questionnaire. Ear and Hearing 1990;11:434-45.

König 2006

König O, Schaette R, Kempter R, Gross M. Course of hearing loss and occurrence of tinnitus. Hearing Research 2006;221:59-64.

Landry 2019

Landry EC, Sandoval XCR, Simeone CN, Tidball G, Lea J, Westerberg BD. Systematic review and network meta-analysis of cognitive and/or behavioral therapies (CBT) for tinnitus. Otology \& Neurotology 2019 Nov 15 [Epub ahead of print].

Langers 2012

Langers DRM, de Kleine E, van Dijk P. Tinnitus does not require macroscopic tonotopic map reorganization. Frontiers in Systems Neuroscience 2012;6:2. [DOI: 10.3389/ fnsys.2012.00002]

Langguth 2011

Langguth B. A review of tinnitus symptoms beyond 'ringing in the ears': a call to action. Current Medical Research and Opinion 2011;27(8):1635-43.

Langguth 2013

Langguth B, Kreuzer PM, Kleinjung T, De Ridder D. Tinnitus: causes and clinical management. Lancet Neurology 2013;12(9):920-30.

Lethem 1983

Lethem J, Slade PD, Troup JD, Bentley G. Outline of a fear-avoidance model of exaggerated pain perception--I. Behaviour Research and Therapy 1983;21(4):4018. [PubMed: 6626110]

Lewinsohn 1978

Lewinsohn PM, Munoz R, Youngren MA, Zeiss AM. Control Your Depression. New Jersey: Prentice-Hall, 1978.

Lovibond 1995

Lovibond SH, Lovibond PF. Manual for the Depression Anxiety Stress Scales. Sydney, Australia: Psychology Foundation, 1995. 
Maes 2014

Maes IH, Cima RF, Anteunis LJ, Scheijen DJ, Baguley DM, El Refaie A, et al. Costeffectiveness of specialized treatment based on cognitive behavioral therapy versus usual care for tinnitus. Otology \& Neurotology 2014;35(5):787-95. [PubMed: 24829038]

Marciano 2003

Marciano E, Varrabba L, Giannini P, Sementina C, Verde P, Bruno C, et al. Psychiatric comorbidity in a population of outpatients affected by tinnitus. International Journal of Audiology 2003;42:4-9.

Martines 2010

Martines F, Bentivegna D, Di Piazza F, Martines E, Sciacca V, Martinciglio G. Investigation of tinnitus patients in Italy: clinical and audiological characteristics. International Journal of Otolaryngology 2010 Jun 23 [Epub ahead of print].

Martinez-Devesa 2010

Martinez-Devesa P, Perera R, Theodoulou M, Waddell A. Cognitive behavioural therapy for tinnitus. Cochrane Database of Systematic Reviews 2010, Issue 9. Art. No.: CD005233. DOI: 10.1002/14651858.CD005233.pub3.

Mazurek 2015

Mazurek B, Szczepek AJ, Hebert S. Stress and tinnitus. HNO 2015;63(4):258-65.

McDermott 1998

McDermott HJ, Lech M. Loudness perception and frequency discrimination in subjects with steeply sloping hearing loss: possible correlates of neural plasticity. Journal of the Acoustical Society of America 1998;104:2314-25.

McKenna 2004

McKenna L. Models of tinnitus suffering and treatment compared and contrasted. Audiological Medicine 2004;2:41-53.

McKenna 2014

McKenna L, Handscomb L, Hoare DJ, Hall DA. A scientific cognitive-behavioral model of tinnitus: novel conceptualizations of tinnitus distress. Frontiers in Neurology 2014;5:196. [PubMed: 25339938]

Mehta 2019

Mehta S, Peynenburg VA, Hadjistavropoulos HD. Internet-delivered cognitive behaviour therapy for chronic health conditions: a systematic review and meta-analysis. Journal of Behavioral Medicine 2019;42:169-87.

Meijerink 2017

Meijerink JF, Pronk M, Paulissen B, Witte BI, Wouden BV, Jansen V, et al. Effectiveness of an online SUpport PRogramme (SUPR) for older hearing aid users: study protocol for a cluster randomised controlled trial. BMJ Open 2017;7(5):e.015012. [Other: ]

Meikle 2012

Meikle MB, Henry JA, Griest SE, Stewart BJ, Abrams HB, McArdle R, et al. The Tinnitus Functional Index: development of a new clinical measure for chronic, intrusive tinnitus. Ear and Hearing 2012;33:153-76. 
Michie 2013

Michie S, Richardson M, Johnston M, Abraham C, Francis J, Hardeman W, et al. The behavior change technique taxonomy (v1) of 93 hierarchically clustered techniques: building an international consensus for the reporting of behavior change interventions. Annals of Behavioral Medicine 2013;46(1):81-95.

Middleton 2011

Middleton JW, Kiritanid T, Pedersen C, Turner JG, Shepherd GMG, Tzounopoulos T. Mice with behavioral evidence of tinnitus exhibit dorsal cochlear nucleus hyperactivity because of decreased GABAergic inhibition. Proceedings of the National Academy of Science 2011;108:7601-6.

Milerova 2013

Milerova J, Anders M, Dvorak T, Sand PG, Koniger S, Langguth B. The influence of psychological factors on tinnitus severity. General Hospital Psychiatry 2013;35(4):412-6.

Moher 2009

Moher D, Liberati A, Tetzlaff J, Altman DG, The PRISMA Group. Preferred Reporting Items for Systematic Reviews and Meta-Analyses: The PRISMA Statement. PLoS Medicine 2009;6(7):e1000097.

Moore 2009

Moore BCJ, Vinay SN. Enhanced discrimination of low-frequency sounds for subjects with high-frequency dead regions. Brain 2009;132:524-36.

Mulders 2010

Mulders WH, Seluakumaran K, Robertson D. Efferent pathways modulate hyperactivity in inferior colliculus. Journal of Neuroscience 2010;30:9578-87.

Munoz 1993

Munoz R, Miranda J. Group Therapy Manual for Cognitive Behavioral Treatment for Depression. Unpublished, 1993.

Møller 2000

Møller AR. Similarities between severe tinnitus and chronic pain. Journal of the American Academy of Audiology 2000;11:115-24.

Mühlnickel 1998

Mühlnickel W, Elbert T, Taub E, Flor H. Reorganization of auditory cortex in tinnitus (plasticity magnetic source imaging). Proceedings of the National Academy of Sciences of the United States of America 1998;95:10340-3.

Newman 1996

Newman CW, Jacobson GP, Spitzer JB. Development of the Tinnitus Handicap Inventory. Archives of Otolaryngology--Head and Neck Surgery 1996;122:143-8.

Noreña 2005

Noreña AJ, Eggermont JJ. Enriched acoustic environment after noise trauma reduces hearing loss and prevents cortical map reorganization. Journal of Neuroscience 2005;25:699-705. 
Noreña 2011

Noreña AJ. An integrative model of tinnitus based on a central gain controlling neural sensitivity. Neuroscience and Biobehavioral Reviews 2011;35:1089-109.

Nyenhuis 2013b

Nyenhuis N, Golm D, Kroner-Herwig B. A systematic review and meta-analysis on the efficacy of self-help interventions in tinnitus. Cognitive Behaviour Therapy 2013;42(2):159-69.

Ost 1987

Ost LG. Applied relaxation: description of a coping technique and review of controlled studies. Behaviour Research and Therapy 1987;25(5):397-409. [0005-7967: (Print)]

Pavlov 1927

Pavlov I. Conditioned Reflexes. London: Oxford University Press, 1927.

Philippot 2012

Philippot P, Nef F, Clauw L, de Romree M, Segal Z. A randomized controlled trial of mindfulness-based cognitive therapy for treating tinnitus. Clinical Psychology \& Psychotherapy 2012;19(5):411-9. [PubMed: 21567655]

Phillips 2010

Phillips JS, McFerran D. Tinnitus Retraining Therapy (TRT) for tinnitus. Cochrane Database of Systematic Reviews 2010, Issue 3. Art. No.: CD007330. DOI: 10.1002/14651858.CD007330.pub2.

Pilati 2012

Pilati N, Large C, Forsythe ID, Hamann M. Acoustic over-exposure triggers burst firing in dorsal cochlear nucleus fusiform cells. Hearing Research 2012;283:98-106.

Pitrou 2009

Pitrou I, Boutron I, Ahmad N, Ravaud P. Reporting of safety results in published reports of randomized controlled trials. Archives of Internal Medicine 2009;169(19):1756-61.

Rademaker 2019

Rademaker MM, Stegeman I, Ho-Kang-You KE, Stokroos RJ, Smit AL. The effect of mindfulness-based interventions on tinnitus distress. A systematic review. Frontiers in Neurology 2019;10:1135.

Ratnayake 2009

Ratnayake SA, Jayarajan V, Bartlett J. Could an underlying hearing loss be a significant factor in the handicap caused by tinnitus? Noise and Health 2009;11:156-60.

Rauschecker 1999

Rauschecker JP. Auditory cortical plasticity: a comparison with other sensory systems. Trends in Neurosciences 1999;22:74-80.

Rauschecker 2010

Rauschecker JP, Leaver AM, Mühlau M. Tuning out the noise: limbic-auditory interaction in tinnitus. Neuron 2010;66:819-26. 


\section{Reiss 1986}

Reiss S, Peterson RA, Gursky DM, McNally RJ. Anxiety sensitivity, anxiety frequency and the prediction of fearfulness. Behaviour Research and Therapy 1986;24(1):1-8.

RevMan 2014

Review Manager (RevMan) [Computer program]. Version 5.3. Copenhagen: The Nordic Cochrane Centre, The Cochrane Collaboration, 2014.

Rief 2005

Rief W, Weise C, Kley N, Martin A. Psychophysiologic treatment of chronic tinnitus: a randomized clinical trial. Psychosomatic Medicine 2005;67:833-8.

Roberts 2010

Roberts L, Eggermont J, Caspary D, Shore S, Melcher J, Kaltenbach J. Ringing ears: the neuroscience of tinnitus. Journal of Neuroscience 2010;30:14972-9.

Sanchez 2002

Sanchez TG, Ferrari GMS. The control of tinnitus through hearing aids: suggestions for optimal use [O controle do zumbido por meio de prótese auditiva: sugestões para otimização do uso]. Pró-Fono Revista de Atualização Científica 2002;14:111-8.

Schaette 2011

Schaette R, McAlpine D. Tinnitus with a normal audiogram: physiological evidence for hidden hearing loss and computational model. Journal of Neuroscience 2011;31:13452-7.

Schecklmann 2013

Schecklmann M, Lehner A, Poeppl TB, Kreuzer PM, Rupprecht R, Rackl J, et al. Auditory cortex is implicated in tinnitus distress: a voxel-based morphometry study. Brain Structure \& Function 2013;218(4):1061-70.

Schork 2015

Schork N. Personalized medicine: time for one-person trials. Nature 2015;520:609-11. [DOI: 10.1038/520609]

Schulz 2010

Schulz KF, Altman DG, Moher D. CONSORT 2010 Statement: updated guidelines for reporting parallel group randomised trials. BMJ 2010;340:c332.

Segal 2012

Segal ZV, Williams JM, Teasdale JD. Mindfulness-based Cognitive Therapy for Depression. 2nd edition. New York: Guildford Press, 2012.

Seki 2003

Seki S, Eggermont JJ. Changes in spontaneous firing rate and neural synchrony in cat primary auditory cortex after localized tone-induced hearing loss. Hearing Research 2003;180:28-38.

\section{Sereda 2018}

Sereda M, Xia J, El Refaie A, Hall DA, Hoare DJ. Sound therapy (using amplification devices and/or sound generators) for tinnitus. Cochrane Database of Systematic Reviews 2018, Issue 12. Art. No.: CD013094. DOI: 10.1002/14651858.CD013094.pub2. 
Chapter 3

\section{Shore 2016}

Shore SE, Roberts LE, Langguth B. Maladaptive plasticity in tinnitus triggers, mechanisms and treatment. Nature Reviews. Neurology 2016;12(3):150-60.

Skevington 2004

Skevington SM, Lofty M, O'Connell KA. The World Health Organization's WHOQOLBREF quality of life assessment: psychometric properties and results of the international field trial. A report from the WHOQOL group. Quality of Life Research 2004;13(2):299-310.

Skinner 1938

Skinner BF. The Behavior of Organisms. New York: Appleton-Century-Crofts, 1938.

Spielberger 1983

Spielberger C, Gorsuch R, Lushene R, Vagg P, Jacobs, G. Manual for the State-Trait Anxiety Inventory. Palo Alto, CA: Consulting Psychologists Press, 1983.

Spitzer 2006

Spitzer RL, Kroenke K, Williams JB, Löwe BI. A brief measure for assessing generalized anxiety disorder: the GAD-7. Archives of Internal Medicine 2006;166(10):1092-7.

Sundell 1982

Sundell M, Sundell SS. Behavior Modification in the Human Services. Englewood Cliffs, NJ: Prentice-Hall, 1982.

Sweetow 1990

Sweetow R, Levy M. Tinnitus severity scaling for diagnostic/therapeutic usage. Hearing Instruments 1990;41:20-1.

Tass 2012

Tass PA, Adamchic I, Freund H-J, von Stackelberg T, Hauptmann C. Counteracting tinnitus by acoustic coordinated reset neuromodulation. Restorative Neurology and Neuroscience 2012;30:137-59.

Thai-Van 2002

Thai-Van H, Micheyl C, Noreña A, Collet L. Local improvement in auditory frequency discrimination is associated with hearing loss slope in subjects with cochlear damage. Brain 2002;125:524-37.

Thai-Van 2003

Thai-Van H, Micheyl C, Moore BCJ, Collet L. Enhanced frequency discrimination near the hearing loss cut-off: a consequence of central auditory plasticity induced by cochlear damage? Brain 2003;126:2235-45.

Thompson 2016

Thompson DM, Hall DA, Walker D-M, Hoare DJ. Psychological therapy for people with tinnitus: a scoping review of treatment components. Ear and Hearing 2016 Aug 18 [Epub ahead of print]. 
Tunkel 2014

Tunkel DE, Bauer CA, Sun GH, Rosenfeld RM, Chandrasekhar SS, Cunningham ER, et al. Clinical Practice Guideline: Tinnitus. Otolaryngology - Head and Neck Surgery 2014;151(2 Suppl):S1-40.

\section{Turk 1983}

Turk DC, Meichenbaum D, Genest M. Pain and Behavioural Medicine - A Cognitive Behavioural Perspective. New York: Guilford Press, 1983.

Van Rillaer 1997

Van Rillaer J. Learning relaxation in cognitive behavioural therapy [L'apprentissage de la relaxation en thérapie cognitivocomportementale]. Revue Francophone de Clinique Comportementale et Cognitives 1997;2:16-20.

Vanneste 2012

Vanneste S, De Ridder D. The auditory and non-auditory brain areas involved in tinnitus. An emergent property of multiple parallel overlapping subnetworks. Frontiers in Systems Neuroscience 2012;6:31.

Vlaeyen 2000

Vlaeyen JW, Linton SJ. Fear-avoidance and its consequences in chronic musculoskeletal pain: a state of the art. Pain 2000;85(3):317-32. [PubMed: 10781906]

Vlaeyen 2012

Vlaeyen JW, Linton SJ. Fear-avoidance model of chronic musculoskeletal pain: 12 years on. Pain 2012;153(6):1144-7. [PubMed: 22321917]

Wegner 2018

Wegner I, Hall DA, Smit AL, McFerran D, Stegeman I. Betahistine for tinnitus. Cochrane Database of Systematic Reviews 2018, Issue 12. Art. No.: CD013093. DOI: 10.1002/14651858.CD013093.pub2.

Weise 2013

Weise C, Kleinstäuber M, Hesser H, Westin VZ, Andersson G. Acceptance of tinnitus: validation of the tinnitus acceptance questionnaire. Cognitive Behaviour Therapy 2013;42:100-15.

Weisz 2005

Weisz N, Moratti S, Meinzer M, Dohrmann K, Elbert T. Tinnitus perception and distress is related to abnormal spontaneous brain activity as measured by magnetoencephalography. PLoS Medicine 2005;2:e153.

Wilson 1991

Wilson PH, Henry J, Bowen M, Haralambous G. Tinnitus Reaction Questionnaire: psychometric properties of a measure of distress associated with tinnitus. Journal of Speech and Hearing Research 1991;34:197-201.

Wilson 1998

Wilson PH, Henry JL. Tinnitus Cognitions Questionnaire: development and psychometric properties of a measure of dysfunctional cognitions associated with tinnitus. International Tinnitus Journal 1998;4(1):23-30. 
Zeman 2011

Zeman F, Koller M, Figueiredo R, Aazevedo A, Rates M, Coelho C, et al. Tinnitus Handicap Inventory for evaluating treatment effects: which changes are clinically relevant? Otolaryngology - Head and Neck Surgery 2011;145(2):282-7. [DOI: 10.1177/0194599811403882]

Zigmond 1983

Zigmond AS, Snaith RP. The Hospital Anxiety and Depression Scale. Acta Psychiatrica Scandinavica 1983;67:361-70.

Zirke 2013a

Zirke N, Seydel C, Szczepek AJ, Olze H, Haupt H, Mazurek B. Psychological comorbidity in patients with chronic tinnitus: analysis and comparison with chronic pain, asthma or atopic dermatitis patients. Quality of Life Research 2013;22(2):263-72.

Zirke 2013b

Zirke N, Seydel C, Arsoy D, Klapp BF, Haupt H, Szczepek AJ, et al. Analysis of mental disorders in tinnitus patients performed with Composite International Diagnostic Interview. Quality of Life Research 2013;22(8):2095-104.

\section{Other published versions of this review}

Fuller 2017b

Fuller TE, Cima RFF, Langguth B, Mazurek B, Waddell A, Hoare DJ, et al. Cognitive behavioural therapy for tinnitus [Protocol]. Cochrane Database of Systematic Reviews 2017, Issue 4. Art. No.: CD012614. DOI: 10.1002/14651858.CD012614. 


\section{Data and analyses}

Results Table 1. CBT versus No Intervention/Waiting List Control

\begin{tabular}{|c|c|c|c|c|}
\hline Outcome or Subgroup & Studies & Participants & Statistical Method & Effect Estimate \\
\hline $\begin{array}{l}1.1 \text { Impact of tinnitus on } \\
\text { quality of life at end of } \\
\text { treatment }\end{array}$ & 10 & 537 & $\begin{array}{l}\text { Std. Mean Difference } \\
\text { (IV, Random, 95\% CI) }\end{array}$ & $\begin{array}{l}-0.56[-0.83 \\
-0.30]\end{array}$ \\
\hline $\begin{array}{l}1.2 \text { Serious adverse ef- } \\
\text { fects at end of treatment }\end{array}$ & 7 & 447 & $\begin{array}{l}\text { Risk Ratio (IV, Random, } \\
95 \% \mathrm{Cl})\end{array}$ & $\begin{array}{l}3.00[0.13, \\
69.87]\end{array}$ \\
\hline $\begin{array}{l}1.3 \text { Depression at end of } \\
\text { treatment }\end{array}$ & 8 & 502 & $\begin{array}{l}\text { Std. Mean Difference } \\
\text { (IV, Random, 95\% Cl) }\end{array}$ & $\begin{array}{l}-0.34[-0.60 \\
-0.08]\end{array}$ \\
\hline $\begin{array}{l}1.4 \text { Anxiety at end of } \\
\text { treatment }\end{array}$ & 6 & 429 & $\begin{array}{l}\text { Std. Mean Difference } \\
\text { (IV, Random, 95\% Cl) }\end{array}$ & $\begin{array}{l}-0.45[-0.82, \\
-0.09]\end{array}$ \\
\hline $\begin{array}{l}1.5 \text { Health-related quality } \\
\text { of life at end of treatment }\end{array}$ & 2 & 179 & $\begin{array}{l}\text { Std. Mean Difference } \\
\text { (IV, Random, 95\% Cl) }\end{array}$ & $\begin{array}{l}-0.38[-0.67 \\
-0.08]\end{array}$ \\
\hline $\begin{array}{l}1.6 \text { Negatively biased } \\
\text { interpretations of tinnitus } \\
\text { at end of treatment }\end{array}$ & 2 & 84 & $\begin{array}{l}\text { Std. Mean Difference } \\
\text { (IV, Random, 95\% Cl) }\end{array}$ & $\begin{array}{l}-0.40[-1.25 \\
0.45]\end{array}$ \\
\hline $\begin{array}{l}1.7 \text { Subgroup analysis } \\
\text { (random-effects model): } \\
\text { type of therapy - impact } \\
\text { of tinnitus on quality of } \\
\text { life at end of treatment }\end{array}$ & 10 & 537 & $\begin{array}{l}\text { Std. Mean Difference } \\
\text { (IV, Random, 95\% CI) }\end{array}$ & $\begin{array}{l}-0.56[-0.83 \\
-0.30]\end{array}$ \\
\hline 1.7.1 CBT & 6 & 402 & $\begin{array}{l}\text { Std. Mean Difference } \\
\text { (IV, Random, 95\% Cl) }\end{array}$ & $\begin{array}{l}-0.47[-0.81 \\
-0.13]\end{array}$ \\
\hline 1.7.2 ACT & 2 & 62 & $\begin{array}{l}\text { Std. Mean Difference } \\
\text { (IV, Random, 95\% Cl) }\end{array}$ & $\begin{array}{l}-0.61[-1.53, \\
0.32]\end{array}$ \\
\hline 1.7.3 Cognitive therapy & 1 & 40 & $\begin{array}{l}\text { Std. Mean Difference } \\
\text { (IV, Random, 95\% Cl) }\end{array}$ & $\begin{array}{l}-0.97[-1.63 \\
-0.31]\end{array}$ \\
\hline 1.7.4 Mindfulness & 1 & 33 & $\begin{array}{l}\text { Std. Mean Difference } \\
\text { (IV, Random, 95\% Cl) }\end{array}$ & $\begin{array}{l}-0.67[-1.37 \\
0.04]\end{array}$ \\
\hline $\begin{array}{l}1.8 \text { Subgroup analysis } \\
\text { (fixed-effect model): type } \\
\text { of therapy - impact of } \\
\text { tinnitus on quality of life } \\
\text { at end of treatment }\end{array}$ & 10 & 537 & $\begin{array}{l}\text { Std. Mean Difference } \\
\text { (IV, Fixed, 95\% CI) }\end{array}$ & $\begin{array}{l}-0.53[-0.71 \\
-0.35]\end{array}$ \\
\hline 1.8.1 CBT & 6 & 402 & $\begin{array}{l}\text { Std. Mean Difference } \\
\text { (IV, Fixed, 95\% Cl) }\end{array}$ & $\begin{array}{l}-0.45[-0.65 \\
-0.24]\end{array}$ \\
\hline 1.8.2 ACT & 2 & 62 & $\begin{array}{l}\text { Std. Mean Difference } \\
\text { (IV, Fixed, 95\% CI) }\end{array}$ & $\begin{array}{l}-0.72[-1.24 \\
-0.20]\end{array}$ \\
\hline 1.8.3 Cognitive therapy & 1 & 40 & $\begin{array}{l}\text { Std. Mean Difference } \\
\text { (IV, Fixed, 95\% Cl) }\end{array}$ & $\begin{array}{l}-0.97[-1.63, \\
-0.31]\end{array}$ \\
\hline
\end{tabular}


Chapter 3

Results Table 1. CBT versus No Intervention/Waiting List Control

\begin{tabular}{|c|c|c|c|c|}
\hline Outcome or Subgroup & Studies & Participants & Statistical Method & Effect Estimate \\
\hline 1.8.4 Mindfulness & 1 & 33 & $\begin{array}{l}\text { Std. Mean Difference } \\
\text { (IV, Fixed, 95\% CI) }\end{array}$ & $\begin{array}{l}-0.67[-1.37 \\
0.04]\end{array}$ \\
\hline $\begin{array}{l}1.9 \text { Subgroup analysis } \\
\text { (random-effects model): } \\
\text { mode of delivery - im- } \\
\text { pact of tinnitus on quality } \\
\text { of life at end of treatment }\end{array}$ & 10 & 537 & $\begin{array}{l}\text { Std. Mean Difference } \\
\text { (IV, Random, 95\% Cl) }\end{array}$ & $\begin{array}{l}-0.56[-0.83, \\
-0.30]\end{array}$ \\
\hline 1.9.1 Bibliotherapy & 1 & 72 & $\begin{array}{l}\text { Std. Mean Difference } \\
\text { (IV, Random, 95\% Cl) }\end{array}$ & $\begin{array}{l}-0.39[-0.86, \\
0.08]\end{array}$ \\
\hline 1.9.2 Face-to-face & 7 & 258 & $\begin{array}{l}\text { Std. Mean Difference } \\
\text { (IV, Random, 95\% Cl) }\end{array}$ & $\begin{array}{l}-0.66[-1.09 \\
-0.22]\end{array}$ \\
\hline $\begin{array}{l}\text { 1.9.3 Internet-based } \\
\text { CBT }\end{array}$ & 2 & 207 & $\begin{array}{l}\text { Std. Mean Difference } \\
\text { (IV, Random, 95\% Cl) }\end{array}$ & $\begin{array}{l}-0.51[-0.92 \\
-0.09]\end{array}$ \\
\hline $\begin{array}{l}1.10 \text { Subgroup analysis } \\
\text { (fixed-effect model): } \\
\text { mode of delivery - } \\
\text { impact of tinnitus on } \\
\text { quality of life at end of } \\
\text { treatment }\end{array}$ & 10 & 537 & $\begin{array}{l}\text { Std. Mean Difference } \\
\text { (IV, Fixed, 95\% CI) }\end{array}$ & $\begin{array}{l}-0.53[-0.71 \\
-0.35]\end{array}$ \\
\hline 1.10.1 Bibliotherapy & 1 & 72 & $\begin{array}{l}\text { Std. Mean Difference } \\
\text { (IV, Fixed, 95\% CI) }\end{array}$ & $\begin{array}{l}-0.39[-0.86, \\
0.08]\end{array}$ \\
\hline 1.10.2 Face-to-face & 7 & 258 & $\begin{array}{l}\text { Std. Mean Difference } \\
\text { (IV, Fixed, 95\% CI) }\end{array}$ & $\begin{array}{l}-0.56[-0.82, \\
-0.30]\end{array}$ \\
\hline 1.10.3 Web-based CBT & 2 & 207 & $\begin{array}{l}\text { Std. Mean Difference } \\
\text { (IV, Fixed, 95\% CI) }\end{array}$ & $\begin{array}{l}-0.54[-0.83, \\
-0.26]\end{array}$ \\
\hline $\begin{array}{l}\text { 1.11 Subgroup analysis } \\
\text { (random-effects model): } \\
\text { unit of delivery - impact } \\
\text { of tinnitus on quality of } \\
\text { life at end of treatment }\end{array}$ & 10 & 537 & $\begin{array}{l}\text { Std. Mean Difference } \\
\text { (IV, Random, 95\% Cl) }\end{array}$ & $\begin{array}{l}-0.56[-0.83 \\
-0.30]\end{array}$ \\
\hline 1.11.1 Individual & 4 & 323 & $\begin{array}{l}\text { Std. Mean Difference } \\
\text { (IV, Random, 95\% CI) }\end{array}$ & $\begin{array}{l}-0.57[-0.85, \\
-0.28]\end{array}$ \\
\hline 1.11.2 Group & 6 & 214 & $\begin{array}{l}\text { Std. Mean Difference } \\
\text { (IV, Random, 95\% Cl) }\end{array}$ & $\begin{array}{l}-0.59[-1.07 \\
-0.10]\end{array}$ \\
\hline $\begin{array}{l}1.12 \text { Subgroup analysis } \\
\text { (fixed-effect model): unit } \\
\text { of delivery - impact of } \\
\text { tinnitus on quality of life } \\
\text { at end of treatment }\end{array}$ & 10 & 537 & $\begin{array}{l}\text { Std. Mean Difference } \\
\text { (IV, Fixed, 95\% CI) }\end{array}$ & $\begin{array}{l}-0.53[-0.71 \\
-0.35]\end{array}$ \\
\hline 1.12.1 Individual & 4 & 323 & $\begin{array}{l}\text { Std. Mean Difference } \\
\text { (IV, Fixed, 95\% CI) }\end{array}$ & $\begin{array}{l}-0.57[-0.79, \\
-0.34]\end{array}$ \\
\hline
\end{tabular}


Results Table 1. CBT versus No Intervention/Waiting List Control

\begin{tabular}{|c|c|c|c|c|}
\hline Outcome or Subgroup & Studies & Participants & Statistical Method & Effect Estimate \\
\hline 1.12.2 Group & 6 & 214 & $\begin{array}{l}\text { Std. Mean Difference } \\
\text { (IV, Fixed, 95\% Cl) }\end{array}$ & $\begin{array}{l}-0.47[-0.75 \\
-0.19]\end{array}$ \\
\hline $\begin{array}{l}1.13 \text { Subgroup analysis } \\
\text { (random-effects model): } \\
\text { who delivers CBT - im- } \\
\text { pact of tinnitus on quality } \\
\text { of life at end of treatment }\end{array}$ & 10 & 537 & $\begin{array}{l}\text { Std. Mean Difference } \\
\text { (IV, Random, 95\% CI) }\end{array}$ & $\begin{array}{l}-0.56[-0.83 \\
-0.30]\end{array}$ \\
\hline 1.13.1 Psychologist & 4 & 185 & $\begin{array}{l}\text { Std. Mean Difference } \\
\text { (IV, Random, 95\% Cl) }\end{array}$ & $\begin{array}{l}-0.52[-1.11 \\
0.08]\end{array}$ \\
\hline 1.13.2 Bibliotherapy & 1 & 72 & $\begin{array}{l}\text { Std. Mean Difference } \\
\text { (IV, Random, 95\% Cl) }\end{array}$ & $\begin{array}{l}-0.39[-0.86, \\
0.08]\end{array}$ \\
\hline 1.13.3 Other clinician & 3 & 73 & $\begin{array}{l}\text { Std. Mean Difference } \\
\text { (IV, Random, 95\% Cl) }\end{array}$ & $\begin{array}{l}-0.89[-1.51 \\
-0.27]\end{array}$ \\
\hline 1.13.4 Internet-based & 2 & 207 & $\begin{array}{l}\text { Std. Mean Difference } \\
\text { (IV, Random, 95\% Cl) }\end{array}$ & $\begin{array}{l}-0.51[-0.92 \\
-0.09]\end{array}$ \\
\hline $\begin{array}{l}1.14 \text { Subgroup analysis } \\
\text { (fixed-effect model): who } \\
\text { delivers CBT - impact of } \\
\text { tinnitus on quality of life } \\
\text { at end of treatment }\end{array}$ & 10 & 537 & $\begin{array}{l}\text { Std. Mean Difference } \\
\text { (IV, Fixed, 95\% Cl) }\end{array}$ & $\begin{array}{l}-0.53[-0.71 \\
-0.35]\end{array}$ \\
\hline 1.14.1 Psychologist & 4 & 185 & $\begin{array}{l}\text { Std. Mean Difference } \\
\text { (IV, Fixed, 95\% Cl) }\end{array}$ & $\begin{array}{l}-0.45[-0.75 \\
-0.14]\end{array}$ \\
\hline 1.14.2 Other clinician & 3 & 73 & $\begin{array}{l}\text { Std. Mean Difference } \\
\text { (IV, Fixed, 95\% CI) }\end{array}$ & $\begin{array}{l}-0.87[-1.36, \\
-0.38]\end{array}$ \\
\hline 1.14.3 Computer & 3 & 279 & $\begin{array}{l}\text { Std. Mean Difference } \\
\text { (IV, Fixed, 95\% CI) }\end{array}$ & $\begin{array}{l}-0.50[-0.74, \\
-0.26]\end{array}$ \\
\hline $\begin{array}{l}1.15 \text { Sensitivity analysis } \\
\text { without Malinvaud (high } \\
\text { risk of bias) impact of } \\
\text { tinnitus on quality of life } \\
\text { at end of treatment }\end{array}$ & 9 & 454 & $\begin{array}{l}\text { Std. Mean Difference } \\
\text { (IV, Random, 95\% Cl) }\end{array}$ & $\begin{array}{l}-0.64[-0.88 \\
-0.40]\end{array}$ \\
\hline $\begin{array}{l}1.16 \text { Sensitivity analysis } \\
\text { with optimistic assump- } \\
\text { tion for Malinvaud - im- } \\
\text { pact of tinnitus on quality } \\
\text { of life at end of treatment }\end{array}$ & 10 & 526 & $\begin{array}{l}\text { Std. Mean Difference } \\
\text { (IV, Random, 95\% Cl) }\end{array}$ & $\begin{array}{l}-0.65[-0.85 \\
-0.44]\end{array}$ \\
\hline
\end{tabular}

Results Table 2. CBT versus Audiological Care (Tinnitus Education and Rehabilitation for Hearing Loss)

\begin{tabular}{|c|c|c|c|c|}
\hline Outcome or Subgroup & Studies & Participants & Statistical Method & Effect Estimate \\
\hline $\begin{array}{l}2.1 \text { Impact of tinnitus on qual- } \\
\text { ity of life at end of treatment }\end{array}$ & & 444 & $\begin{array}{l}\text { Mean Difference (IV, } \\
\text { Random, 95\% Cl) }\end{array}$ & $\begin{array}{l}-5.68[-9.74 \\
-1.61]\end{array}$ \\
\hline
\end{tabular}


Chapter 3

Results Table 2. Continued.

\begin{tabular}{lllll}
\hline Outcome or Subgroup & Studies & Participants & Statistical Method & Effect Estimate \\
\hline $\begin{array}{l}\text { 2.2 Serious adverse ef- } \\
\text { fects at end of treatment }\end{array}$ & 2 & 410 & $\begin{array}{l}\text { Risk Ratio (M-H, Fixed, } \\
95 \% \mathrm{Cl})\end{array}$ & Not estimable \\
\hline $\begin{array}{l}2.3 \text { Depression at end of } \\
\text { treatment }\end{array}$ & 2 & 410 & $\begin{array}{l}\text { Std. Mean Difference } \\
\text { (IV, Random, 95\% Cl) }\end{array}$ & $\begin{array}{l}-0.18[-0.38, \\
0.01]\end{array}$ \\
\hline $\begin{array}{l}2.4 \text { Anxiety at end of } \\
\text { treatment }\end{array}$ & 2 & 410 & $\begin{array}{l}\text { Std. Mean Difference } \\
\text { (IV, Random, 95\% Cl) }\end{array}$ & $-0.06[-0.26$, \\
\hline $\begin{array}{l}2.5 \text { Health-related quality } \\
\text { of life }\end{array}$ & 2 & 410 & $\begin{array}{l}\text { Std. Mean Difference } \\
\text { (IV, Random, 95\% Cl) }\end{array}$ & $-0.07[-0.26$, \\
\hline $\begin{array}{l}2.6 \text { Negatively biased } \\
\text { interpretations of tinnitus }\end{array}$ & 1 & 336 & Mean Difference (IV, & $-4.69[-7.04$, \\
\hline $\begin{array}{l}2.7 \text { Sensitivity analysis } \\
\text { (fixed-effect model): impact } \\
\text { of tinnitus on quality of life }\end{array}$ & 3 & 430 & Random, 95\% Cl) & $-2.34]$ \\
\hline
\end{tabular}

Results Table 3. CBT versus TRT (Directive Counselling and Bilateral Masking)

\begin{tabular}{|c|c|c|c|c|}
\hline Outcome or Subgroup & Studies & Participants & Statistical Method & Effect Estimate \\
\hline $\begin{array}{l}3.1 \text { Impact of tinnitus on } \\
\text { quality of life at end of } \\
\text { treatment }\end{array}$ & 1 & 42 & $\begin{array}{l}\text { Mean Difference (IV, } \\
\text { Random, } 95 \% \mathrm{Cl} \text { ) }\end{array}$ & $\begin{array}{l}-15.79[-27.91, \\
-3.67]\end{array}$ \\
\hline $\begin{array}{l}3.2 \text { Impact of tinnitus on } \\
\text { quality of life at } 6 \text { months } \\
\text { follow-up }\end{array}$ & 1 & 42 & $\begin{array}{l}\text { Mean Difference (IV, } \\
\text { Random, 95\% CI) }\end{array}$ & $\begin{array}{l}-13.10[-26.08 \\
-0.12]\end{array}$ \\
\hline $\begin{array}{l}\text { 3.3 Serious adverse effects } \\
\text { at end of treatment }\end{array}$ & 1 & 42 & $\begin{array}{l}\text { Risk Ratio (IV, Ran- } \\
\text { dom, 95\% CI) }\end{array}$ & $0.45[0.04,4.64]$ \\
\hline $\begin{array}{l}3.4 \text { Negatively biased inter- } \\
\text { pretations of tinnitus at end } \\
\text { of treatment }\end{array}$ & 1 & 42 & $\begin{array}{l}\text { Mean Difference (IV, } \\
\text { Random, 95\% Cl) }\end{array}$ & $\begin{array}{l}-9.78[-16.40 \\
-3.16]\end{array}$ \\
\hline $\begin{array}{l}3.5 \text { Negatively biased } \\
\text { interpretations of tinnitus at } \\
6 \text { months follow-up }\end{array}$ & 1 & 42 & $\begin{array}{l}\text { Mean Difference (IV, } \\
\text { Random, 95\% Cl) }\end{array}$ & $\begin{array}{l}-8.28[-15.34, \\
-1.22]\end{array}$ \\
\hline
\end{tabular}

Results Table 4. CBT versus Other Active Control

\begin{tabular}{lllll}
\hline Outcome or Subgroup & Studies & Participants & Statistical Method & Effect Estimate \\
\hline $\begin{array}{l}4.1 \text { Impact of tinnitus on } 12 \\
\text { quality of life at end of }\end{array}$ & 966 & $\begin{array}{l}\text { Std. Mean Difference } \\
\text { (IV, Random, 95\% Cl) }\end{array}$ & $\begin{array}{l}-0.30[-0.55, \\
\text { treatment }\end{array}$ \\
\hline $\begin{array}{l}4.2 \text { Impact of tinnitus on } 1 \\
\begin{array}{l}\text { quality of life at } 6 \text { months } \\
\text { follow-up }\end{array}\end{array}$ & 62 & $\begin{array}{l}\text { Mean Difference } \\
\text { (IV, Random, 95\% Cl) }\end{array}$ & $-0.54]$ \\
\hline
\end{tabular}


Results Table 4. Continued.

\begin{tabular}{|c|c|c|c|c|}
\hline Outcome or Subgroup & Studies & Participants & Statistical Method & Effect Estimate \\
\hline $\begin{array}{l}4.3 \text { Impact of tinnitus } \\
\text { on quality of life at } 12 \\
\text { months follow-up }\end{array}$ & 1 & 33 & $\begin{array}{l}\text { Mean Difference } \\
(\mathrm{IV}, \text { Random, } 95 \% \mathrm{Cl})\end{array}$ & $-2.76[-14.69,9.17]$ \\
\hline $\begin{array}{l}4.4 \text { Serious adverse } \\
\text { effects at the end of } \\
\text { treatment }\end{array}$ & 6 & 595 & $\begin{array}{l}\text { Risk Ratio (M-H, } \\
\text { Random, 95\% Cl) }\end{array}$ & $1.70[0.16,18.36]$ \\
\hline $\begin{array}{l}4.5 \text { Depression at end of } \\
\text { treatment }\end{array}$ & 11 & 943 & $\begin{array}{l}\text { Std. Mean Difference } \\
\text { (IV, Random, 95\% CI) }\end{array}$ & $-0.17[-0.33,-0.01]$ \\
\hline $\begin{array}{l}\text { 4.6 Depression at } 6 \\
\text { months follow-up }\end{array}$ & 1 & 62 & $\begin{array}{l}\text { Mean Difference } \\
\text { (IV, Random, 95\% Cl) }\end{array}$ & $-1.90[-3.87,0.07]$ \\
\hline $\begin{array}{l}\text { 4.7 Depression at } 12 \\
\text { months }\end{array}$ & 1 & 33 & $\begin{array}{l}\text { Mean Difference } \\
(\mathrm{IV}, \text { Random, } 95 \% \mathrm{Cl})\end{array}$ & $-2.00[-7.88,3.88]$ \\
\hline $\begin{array}{l}4.8 \text { Anxiety at end of } \\
\text { treatment }\end{array}$ & 9 & 696 & $\begin{array}{l}\text { Std. Mean Difference } \\
\text { (IV, Random, 95\% Cl) }\end{array}$ & $\begin{array}{l}-0.25[-0.48 \\
-0.02]\end{array}$ \\
\hline $\begin{array}{l}4.9 \text { Anxiety at } 6 \text { months } \\
\text { follow-up }\end{array}$ & 1 & 62 & $\begin{array}{l}\text { Mean Difference } \\
\text { (IV, Random, 95\% Cl) }\end{array}$ & $-1.20[-3.07,0.67]$ \\
\hline $\begin{array}{l}4.10 \text { Health-related } \\
\text { quality of life at end of } \\
\text { treatment }\end{array}$ & 1 & 95 & $\begin{array}{l}\text { Mean Difference } \\
\text { (IV, Random, 95\% CI) }\end{array}$ & $-0.05[-0.68,0.59]$ \\
\hline $\begin{array}{l}4.11 \text { Negatively biased } \\
\text { interpretations of tinnitus } \\
\text { at end of treatment }\end{array}$ & 5 & 455 & $\begin{array}{l}\text { Std. Mean Difference } \\
\text { (IV, Random, 95\% Cl) }\end{array}$ & $\begin{array}{l}-0.55[-0.75 \\
-0.35]\end{array}$ \\
\hline $\begin{array}{l}4.12 \text { Negatively biased } \\
\text { interpretations of tinnitus } \\
\text { at } 6 \text { months follow-up }\end{array}$ & 1 & 62 & $\begin{array}{l}\text { Mean Difference } \\
\text { (IV, Random, 95\% Cl) }\end{array}$ & $\begin{array}{l}-7.20[-13.65 \\
-0.75]\end{array}$ \\
\hline $\begin{array}{l}4.13 \text { Negatively biased } \\
\text { interpretations of tinnitus } \\
\text { at } 12 \text { months follow-up }\end{array}$ & 1 & 33 & $\begin{array}{l}\text { Mean Difference } \\
(\mathrm{IV}, \text { Random, } 95 \% \mathrm{Cl})\end{array}$ & $\begin{array}{l}-14.05[-24.80 \\
-3.30]\end{array}$ \\
\hline $\begin{array}{l}4.14 \text { Subgroup analysis } \\
\text { (random-effects model): } \\
\text { type of therapy - impact } \\
\text { of tinnitus on quality of life }\end{array}$ & 12 & 966 & $\begin{array}{l}\text { Std. Mean Difference } \\
\text { (IV, Random, 95\% CI) }\end{array}$ & $\begin{array}{l}-0.30[-0.55 \\
-0.05]\end{array}$ \\
\hline 4.14.1 CBT & 5 & 626 & $\begin{array}{l}\text { Std. Mean Difference } \\
\text { (IV, Random, 95\% Cl) }\end{array}$ & $-0.23[-0.62,0.15]$ \\
\hline 4.14.2 Cognitive therapy & 3 & 97 & $\begin{array}{l}\text { Std. Mean Difference } \\
\text { (IV, Random, } 95 \% \mathrm{Cl} \text { ) }\end{array}$ & $-0.38[-0.92,0.17]$ \\
\hline 4.14.3 ACT & 2 & 114 & $\begin{array}{l}\text { Std. Mean Difference } \\
\text { (IV, Random, } 95 \% \mathrm{Cl} \text { ) }\end{array}$ & $-0.20[-1.60,1.21]$ \\
\hline 4.14.4 Mindfulness & 2 & 129 & $\begin{array}{l}\text { Std. Mean Difference } \\
\text { (IV, Random, 95\% Cl) }\end{array}$ & $\begin{array}{l}-0.35[-0.70 \\
-0.00]\end{array}$ \\
\hline
\end{tabular}


Chapter 3

Results Table 4. Continued.

\begin{tabular}{|c|c|c|c|c|}
\hline Outcome or Subgroup & Studies & Participants & Statistical Method & Effect Estimate \\
\hline $\begin{array}{l}4.15 \text { Subgroup analysis } \\
\text { (fixed-effect model): type } \\
\text { of therapy - impact of } \\
\text { tinnitus on quality of life }\end{array}$ & 12 & 966 & $\begin{array}{l}\text { Std. Mean Difference } \\
\text { (IV, Fixed, 95\% CI) }\end{array}$ & $-0.33[-0.46,-0.19]$ \\
\hline 4.15.1 CBT & 5 & 626 & $\begin{array}{l}\text { Std. Mean Difference } \\
\text { (IV, Fixed, 95\% CI) }\end{array}$ & $-0.27[-0.44,-0.10]$ \\
\hline 4.15.2 Cognitive therapy & 3 & 97 & $\begin{array}{l}\text { Std. Mean Difference } \\
\text { (IV, Fixed, 95\% CI) }\end{array}$ & $-0.40[-0.82,0.02]$ \\
\hline 4.15.3 ACT & 2 & 114 & $\begin{array}{l}\text { Std. Mean Difference } \\
\text { (IV, Fixed, 95\% CI) }\end{array}$ & $-0.59[-0.99,-0.19]$ \\
\hline 4.15.4 Mindfulness & 2 & 129 & $\begin{array}{l}\text { Std. Mean Difference } \\
\text { (IV, Fixed, 95\% CI) }\end{array}$ & $\begin{array}{l}-0.35[-0.70, \\
-0.00]\end{array}$ \\
\hline $\begin{array}{l}\text { 4.16 Subgroup analysis: } \\
\text { mode of delivery - impact } \\
\text { of tinnitus on quality of life }\end{array}$ & 12 & 966 & $\begin{array}{l}\text { Std. Mean Difference } \\
\text { (IV, Random, 95\% Cl) }\end{array}$ & $\begin{array}{l}-0.31[-0.55 \\
-0.08]\end{array}$ \\
\hline 4.16.1 Face-to-face & 9 & 633 & $\begin{array}{l}\text { Std. Mean Difference } \\
\text { (IV, Random, 95\% CI) }\end{array}$ & $-0.20[-0.43,0.03]$ \\
\hline $\begin{array}{l}4.16 .2 \text { Internet-based } \\
\text { CBT }\end{array}$ & 4 & 333 & $\begin{array}{l}\text { Std. Mean Difference } \\
\text { (IV, Random, 95\% CI) }\end{array}$ & $\begin{array}{l}-0.52[-0.98, \\
-0.07]\end{array}$ \\
\hline $\begin{array}{l}4.17 \text { Subgroup analysis: } \\
\text { unit of delivery - impact of } \\
\text { tinnitus on quality of life }\end{array}$ & 12 & 966 & $\begin{array}{l}\text { Std. Mean Difference } \\
\text { (IV, Random, 95\% Cl) }\end{array}$ & $\begin{array}{l}-0.31[-0.55 \\
-0.08]\end{array}$ \\
\hline 4.17.1 Individual & 6 & 424 & $\begin{array}{l}\text { Std. Mean Difference } \\
\text { (IV, Random, 95\% Cl) }\end{array}$ & $-0.45[-0.79,-0.11]$ \\
\hline 4.17.2 Group & 7 & 542 & $\begin{array}{l}\text { Std. Mean Difference } \\
\text { (IV, Random, 95\% Cl) }\end{array}$ & $-0.19[-0.48,0.10]$ \\
\hline $\begin{array}{l}\text { 4.18 Subgroup analysis: } \\
\text { who delivers intervention } \\
\text { - impact of tinnitus on } \\
\text { quality of life }\end{array}$ & 12 & 966 & $\begin{array}{l}\text { Std. Mean Difference } \\
\text { (IV, Random, 95\% Cl) }\end{array}$ & $\begin{array}{l}-0.31[-0.55 \\
-0.08]\end{array}$ \\
\hline 4.18.1 Psychologists & 8 & 669 & $\begin{array}{l}\text { Std. Mean Difference } \\
\text { (IV, Random, 95\% Cl) }\end{array}$ & $-0.29[-0.61,0.02]$ \\
\hline 4.18.2 Internet-based & 3 & 209 & $\begin{array}{l}\text { Std. Mean Difference } \\
\text { (IV, Random, 95\% Cl) }\end{array}$ & $-0.40[-1.03,0.23]$ \\
\hline 4.18.3 Other 'therapists' & 2 & 88 & $\begin{array}{l}\text { Std. Mean Difference } \\
\text { (IV, Random, 95\% Cl) }\end{array}$ & $-0.25[-0.68,0.18]$ \\
\hline $\begin{array}{l}4.19 \text { Subgroup analysis: } \\
\text { type of control - impact of } \\
\text { tinnitus on quality of life }\end{array}$ & 12 & 965 & $\begin{array}{l}\text { Std. Mean Difference } \\
\text { (IV, Random, 95\% Cl) }\end{array}$ & $\begin{array}{l}-0.30[-0.54 \\
-0.06]\end{array}$ \\
\hline 4.19.1 Information & 3 & 171 & $\begin{array}{l}\text { Std. Mean Difference } \\
\text { (IV, Random, 95\% Cl) }\end{array}$ & $-0.32[-0.91,0.27]$ \\
\hline
\end{tabular}


Results Table 4. Continued.

\begin{tabular}{|c|c|c|c|c|}
\hline Outcome or Subgroup & Studies & Participants & Statistical Method & Effect Estimate \\
\hline $\begin{array}{l}\text { 4.19.2 Coping effective- } \\
\text { ness training }\end{array}$ & 1 & 19 & $\begin{array}{l}\text { Std. Mean Difference } \\
\text { (IV, Random, 95\% Cl) }\end{array}$ & $0.58[-0.34,1.51]$ \\
\hline 4.19.3 Relaxation & 3 & 159 & $\begin{array}{l}\text { Std. Mean Difference } \\
\text { (IV, Random, 95\% Cl) }\end{array}$ & $-0.31[-0.63,0.01]$ \\
\hline 4.19.4 Discussion forum & 3 & 347 & $\begin{array}{l}\text { Std. Mean Difference } \\
\text { (IV, Random, 95\% Cl) }\end{array}$ & $\begin{array}{l}-0.72[-0.95 \\
-0.49]\end{array}$ \\
\hline 4.19.5 Masking & 1 & 27 & $\begin{array}{l}\text { Std. Mean Difference } \\
\text { (IV, Random, 95\% Cl) }\end{array}$ & $-0.03[-0.83,0.77]$ \\
\hline 4.19.6 Virtual reality & 1 & 116 & $\begin{array}{l}\text { Std. Mean Difference } \\
\text { (IV, Random, 95\% Cl) }\end{array}$ & $0.19[-0.18,0.56]$ \\
\hline 4.19.7 Self-help & 1 & 126 & $\begin{array}{l}\text { Std. Mean Difference } \\
\text { (IV, Random, 95\% Cl) }\end{array}$ & $-0.06[-0.52,0.39]$ \\
\hline $\begin{array}{l}4.20 \text { Sensitivity analysis } \\
\text { (fixed-effect model): im- } \\
\text { pact of tinnitus on quality } \\
\text { of life at end of treatment }\end{array}$ & 12 & 966 & $\begin{array}{l}\text { Std. Mean Difference } \\
\text { (IV, Fixed, 95\% CI) }\end{array}$ & $-0.33[-0.46,-0.19]$ \\
\hline $\begin{array}{l}4.21 \text { Sensitivity analysis: } \\
\text { without studies at high } \\
\text { risk of bias for incom- } \\
\text { plete outcome data } \\
\text { - impact of tinnitus on } \\
\text { quality of life }\end{array}$ & 9 & 770 & $\begin{array}{l}\text { Std. Mean Difference } \\
\text { (IV, Random, 95\% Cl) }\end{array}$ & $-0.48[-0.71,-0.26]$ \\
\hline $\begin{array}{l}4.22 \text { Sensitivity analy- } \\
\text { sis: without high risk of } \\
\text { bias of missing outcome } \\
\text { data, by subgroups (ran- } \\
\text { dom-effects model): type } \\
\text { of therapy - impact of } \\
\text { tinnitus on quality of life }\end{array}$ & 9 & 769 & $\begin{array}{l}\text { Std. Mean Difference } \\
\text { (IV, Random, 95\% Cl) }\end{array}$ & $-0.45[-0.70,-0.21]$ \\
\hline 4.22.1 CBT & 3 & 459 & $\begin{array}{l}\text { Std. Mean Difference } \\
\text { (IV, Random, 95\% Cl) }\end{array}$ & $-0.49[-0.87,-0.12]$ \\
\hline 4.22.2 Cognitive therapy & 2 & 67 & $\begin{array}{l}\text { Std. Mean Difference } \\
\text { (IV, Random, 95\% Cl) }\end{array}$ & $-0.48[-1.31,0.34]$ \\
\hline 4.22.3 ACT & 2 & 114 & $\begin{array}{l}\text { Std. Mean Difference } \\
\text { (IV, Random, 95\% Cl) }\end{array}$ & $-0.20[-1.60,1.21]$ \\
\hline 4.22.4 Mindfulness & 2 & 129 & $\begin{array}{l}\text { Std. Mean Difference } \\
\text { (IV, Random, 95\% Cl) }\end{array}$ & $\begin{array}{l}-0.35[-0.70 \\
-0.00]\end{array}$ \\
\hline $\begin{array}{l}\text { 4.23 Sensitivity analysis: } \\
\text { optimistic assumption } \\
\text { for Malinvaud - impact of } \\
\text { tinnitus on quality of life }\end{array}$ & 12 & 955 & $\begin{array}{l}\text { Std. Mean Difference } \\
\text { (IV, Random, 95\% Cl) }\end{array}$ & $-0.35[-0.57,-0.13]$ \\
\hline
\end{tabular}


Chapter 3

Results Table 4. Continued.

\begin{tabular}{|c|c|c|c|c|}
\hline Outcome or Subgroup & Studies & Participants & Statistical Method & Effect Estimate \\
\hline $\begin{array}{l}\text { 4.24 Sensitivity analysis: } \\
\text { optimistic assumption } \\
\text { for Malinvaud - depres- } \\
\text { sion }\end{array}$ & 11 & 921 & $\begin{array}{l}\text { Std. Mean Difference } \\
\text { (IV, Random, 95\% Cl) }\end{array}$ & $\begin{array}{l}-0.21[-0.34 \\
-0.07]\end{array}$ \\
\hline $\begin{array}{l}\text { 4.25 Sensitivity analysis: } \\
\text { optimistic assumption } \\
\text { for Malinvaud - anxiety }\end{array}$ & 9 & 674 & $\begin{array}{l}\text { Std. Mean Difference } \\
\text { (IV, Random, 95\% Cl) }\end{array}$ & $\begin{array}{l}-0.28[-0.48 \\
-0.09]\end{array}$ \\
\hline
\end{tabular}

\section{Sources of support}

Internal sources

No sources of support provided

\section{External sources}

National Institute for Health Research, UK

Infrastructure funding for Cochrane ENT

SWOL Limburgs Fonds voor Revalidatie and Netherlands Organisation for Health Research and Development (ZonMW), Netherlands

Research programme: Health Care Efficiency, Subprogramme: Effects \& Costs, Grant number: 945-07-715 provided funding for the employment of Thomas Fuller 


\section{Appendices}

\section{Pathophysiology of tinnitus}

In the central auditory system, tinnitus-related alterations have been described along the whole central auditory pathway including the dorsal cochlear nucleus (Middleton 2011; Pilati 2012), the inferior colliculus (Dong 2010; Mulders 2010), and the auditory and non-auditory cortex (for review see Elgoyhen 2015). There is a strong rationale that these structural and functional alterations are a direct consequence of maladaptive neuroplastic responses to hearing loss (Møller 2000; Mühlnickel 1998), or to altered somatosensory input from the face or the neck (Shore 2016). Presumably sensory deafferentation triggers a release from inhibition in the central auditory system resulting in spontaneous hyperactivity and increased spontaneous synchronous activity within the central neuronal networks involved in sound processing (Dietrich 2001; Eggermont 2004; Rauschecker 1999; Schaette 2011; Seki 2003; Tass 2012; Weisz 2005). Another physiological change thought to be related to tinnitus generation is a process of functional reorganisation. This amounts to a change in the response properties of neurons within the primary auditory cortex to external sounds. This effect is well demonstrated physiologically in animal models of hearing loss (Engineer 2011; Noreña 2005). Evidence in humans, however, is limited to behavioural evidence of cortical reorganisation after hearing loss, demonstrating improved frequency discrimination ability at the audiometric edge (Kluk 2006; McDermott 1998; Moore 2009; Thai-Van 2002; Thai-Van 2003), although Buss 1998 did not find this effect. Imaging studies in tinnitus patients without hearing loss as shown in a normal audiogram did not demonstrate functional reorganisation of the brain's auditory system macroscopically altered tonotopic organisation (Langers 2012), indicating that altered tonotopic organisation is rather a consequence of hearing loss and not causally related to tinnitus. This indicates that such reorganisation is a consequence of hearing loss, but is not sufficient to cause tinnitus.

\section{Psychological models of the effects of tinnitus}

Several influential models have been proposed to explain the development and maintenance of distress and interference associated with chronic subjective tinnitus. Each of the models are briefly described here as they underlie the development of and rationale for applying cognitive behavioural therapy for the treatment of the impact of tinnitus on quality of life.

The concept of habituation - a process whereby reaction(s) decrease in response to repeated presentation of a stimulus (Bouton 2007) - was first applied in 1984 by Hallam and colleagues to explain reduction in the impact of tinnitus on quality of life over time. They proposed that for most people repeated perception of the tinnitus sound led them to learn that the stimulus was not worthy of attentional resources (Hallam 1984). However, lower tinnitus-related quality of life occurs when there are failures in these attentional processes that might especially happen at times of stress and high arousal, which put strain on cognitive resources (Mazurek 2015). Operant conditioning (Skinner 1938), which attributes importance to the consequences of actions, was later included in the model to account for learning mechanisms and avoidant behaviours (Kröner-Herwig 2003). The 
difficulty for the person arises though when significant or continuous resources (cognitive or otherwise) are needed to avoid the tinnitus to experience relief. To treat the impact of tinnitus on quality of life (or facilitate habituation to tinnitus), it was recommended that stress levels and central nervous system arousal levels should be reduced in order to change the meaning of the tinnitus signal for the patient (McKenna 2004). To date there is mixed evidence in support of the habituation model (Baguley 2013).

Jastreboff expanded this model by postulating that the association between tinnitus and an aversive emotional state emerges through classical conditioning mechanisms (Jastreboff 1988; Jastreboff 1990). Classical (or Pavlovian) conditioning refers to a process whereby a person learns a relationship between the two stimuli, a neutral one (conditioned stimulus) and a biologically relevant one (unconditioned stimulus) (Pavlov 1927). Subsequent presentation of either will activate the representation of the biologically relevant one and elicit a conditioned response. While Jastreboff described how an association developed between the tinnitus perception and an aversive emotional state, it was not clearly specified what the unconditioned stimulus, conditioned stimulus and conditioned responses respectively were (Baguley 2013). Regardless, to counter the effect, treatment should aim to break the negative association with the tinnitus percept by using directive cognitive therapy and sound therapy (Jastreboff 1993; Jastreboff 2004).

More recently a cognitive model (McKenna 2014), and cognitive-behavioural (i.e. fear avoidance) model (Cima 2011b; Kleinstauber 2013; Lethem 1983; Vlaeyen 2000; Vlaeyen 2012), have been applied to tinnitus. The cognitive model stresses the importance of primary and secondary cognitive appraisals and the effect on attentional processes (McKenna 2014). The negative evaluation of the tinnitus can be viewed as being comprised of primary and secondary appraisals. That is, a person might initially appraise the tinnitus as being threatening to their health, and then make a secondary appraisal of their (in) ability to control it. The fear avoidance model of tinnitus shares features with both the neurophysiological and the cognitive model including attributing a fundamental role to the negative evaluation of tinnitus. The fear avoidance model offers predictions about behavioural factors (e.g. safety behaviours) in the maintenance of lower tinnitus related quality of life. It is proposed that regardless of the cause of the tinnitus, once it is detected, attention, cognitive appraisals and emotional reactions elicit behavioural responses, which are relieving in the short term but paradoxically lead to severe impairment in the long term.

In the fear avoidance model, the role of fear reactions and safety behaviours is purported to be the key mechanism in the maintenance of chronic tinnitus suffering (Cima 2011b; Kleinstauber 2013). Its central tenet is that the main reactions to tinnitus depend on the initial response. In case of misinterpretations, increased threat value will be associated 
with tinnitus. That is to say, negative autonomic psychophysiological reactivity may lead to catastrophic (mis)interpretations (i.e. a bias towards misinterpreting the tinnitus as something extremely harmful). Fear responses, such as avoidance and escape tendencies, will in turn lead to task-interference, depression, inactivity and ultimately to severe impairment in daily life (Cima 2011a; Cima 2011b). These fear behaviours are reinforced since they offer relief by reducing fear and acute reactivity in the short term, but unfortunately prolong fear-avoidance responsiveness and therefore impairment in the long term.

Although these psychological models slightly differ in their main premise and in some of the terminology used, they all identify mechanisms, either of a cognitive and/or behavioural nature, which have been targeted in therapy to tinnitus related quality of life.

\section{Tinnitus measurement tools}

There are numerous tools used for tinnitus evaluation including the Tinnitus Questionnaire (TQ) (Hallam 1988), the Tinnitus Reaction Questionnaire (TRQ) (Wilson 1991), the Tinnitus Functional Index (TFI) (Meikle 2012) and the Tinnitus Handicap Inventory (THI) (Newman 1996). For a discussion of the development and validity of questionnaires for measuring the impact of tinnitus on quality of life see Fackrell 2014.

\section{For illustrative purposes, the THI is presented below.}

The THI is a self-administered tool to measure the impact of the tinnitus in daily life (Newman 1996). It consists of 25 items that may be answered yes (four points), sometimes (two points) or no (zero points), summing up a total of 100 points, with higher scores corresponding to a higher handicap. The items are divided into three subscales:

- The functional subscale (F) (11 items) encompasses role limitations in the areas of mental functioning, social/occupational functioning and physical functioning.

- The emotional subscale (E) (nine items) includes items addressing affective responses to tinnitus (anger, frustration, irritability, depression).

- The catastrophic subscale (C) (five items) reflects patients' desperation, inability to escape from tinnitus, perception of having a terrible disease, lack of control and inability to cope.

1. Because of your tinnitus is it difficult for you to concentrate? (F)

2. Does the loudness of your tinnitus make it difficult for you to hear people? (F)

3. Does your tinnitus make you angry? (E)

4. Does your tinnitus make you confused? (F)

5. Because of your tinnitus are you desperate? (C)

6. Do you complain a great deal about your tinnitus? (E)

7. Because of your tinnitus do you have trouble falling asleep at night? $(\mathrm{F})$

8. Do you feel as though you cannot escape from your tinnitus? (C) 
9. Does your tinnitus interfere with your ability to enjoy social activities (such as going out to dinner, to the cinema)? (F)

10. Because of your tinnitus do you feel frustrated? $(E)$

11. Because of your tinnitus do you feel that you have a terrible disease? (C)

12. Does your tinnitus make it difficult to enjoy life? (F)

13. Does your tinnitus interfere with your job or household responsibilities? $(F)$

14. Because of your tinnitus do you find that you are often irritable? (F)

15. Because of your tinnitus is it difficult for you to read? (F)

16. Does your tinnitus make you upset? (E)

17. Do you feel that your tinnitus has placed stress on your relationships with members of your family and friends? (E)

18. Do you find it difficult to focus your attention away from your tinnitus and on to other things? $(F)$

19. Do you feel that you have no control over your tinnitus? (C)

20. Because of your tinnitus do you often feel tired? $(F)$

21. Because of your tinnitus do you feel depressed? (E)

22. Does your tinnitus make you feel anxious? (E)

23. Do you feel you can no longer cope with your tinnitus? (C)

24. Does your tinnitus get worse when you are under stress? $(\mathrm{F})$

25. Does your tinnitus make you feel insecure? (E)

According to the score, tinnitus can be classified into five categories:

Category 1: 0 to 16. Slight (only heard in quiet environments).

Category 2: 18 to 36 . Mild (easily masked by environmental sounds and easily forgotten with activities).

Category 3: 38 to 56. Moderate (noticed in the presence of background noise, though daily activities can still be performed).

Category 4: 58 to 76. Severe (almost always heard, leads to disturbed sleep patterns and can interfere with daily activities).

Category 5: 78 to 100. Catastrophic (always heard, disturbed sleep patterns, difficulty with any activities).

\section{Outcome measures and citations}

- Tinnitus Questionnaire (Hallam 1988; Hallam 2008).

- German version of Tinnitus Questionnaire (Goebel 1994).

- Tinnitus Functional Index (Meikle 2012).

- Tinnitus Handicap Inventory (Newman 1996).

- Tinnitus Handicap Questionnaire (Kuk 1990).

- Tinnitus Reaction Questionnaire (Wilson 1991).

- Tinnitus Severity Scale (Sweetow 1990).

- Tinnitus Disability Index (Cima 2011a). 


\section{Search strategies}

\begin{tabular}{|c|c|c|}
\hline L (CRS Web) & MEDLINE (Ovid) & Embase (Ovid) \\
\hline $\begin{array}{l}\text { \#1 MESH DESCRIPTOR Tinnitus EX- } \\
\text { PLODE ALL AND CENTRAL:TARGET }\end{array}$ & $\begin{array}{l}\text { 1. } \exp \text { Tinnitus/ } \\
\text { 2. "tinnit"”.ab,ti. }\end{array}$ & $\begin{array}{l}\text { 1. ex } \\
\text { 2. } " \mathrm{t}\end{array}$ \\
\hline \#2 (tinn & 3. exp Behavior Therapy/ & 3. exp behavior therapy/ \\
\hline & 4. exp Adaptation, Psy- & 4. $\exp a d$ \\
\hline \#3 MEs & & 5. $\exp \mathrm{m}$ \\
\hline $\begin{array}{l}\text { DLODE ALL AND CEN- } \\
\text { ET }\end{array}$ & $\begin{array}{l}\text { 5. } \exp \text { Med } \\
\text { 6. (CBT or }\end{array}$ & $\begin{array}{l}\text { or } \\
\text { or MBTR }\end{array}$ \\
\hline $\begin{array}{l}\text { RIPTOR Adaptation, } \\
\text { PLODE ALL AND } \\
\text { ET }\end{array}$ & $\begin{array}{l}\text { or MBTR } \\
\text { MBTSR } \\
\text { ducation }\end{array}$ & $\begin{array}{l}\text { or } \mathrm{N} \\
\text { or } \mathrm{p} \\
\text { or } \mathrm{i}\end{array}$ \\
\hline $\begin{array}{l}\text { CRIPTOR Meditation } \\
\text { AND CENTRAL:TARGET }\end{array}$ & $\begin{array}{l}\text { iCBT or } \\
\text { ti. }\end{array}$ & $\begin{array}{r}\mathrm{GC} \\
\text { 7. }((\mathrm{co}\end{array}$ \\
\hline $\begin{array}{l}\text { \#6 (CBT or ACT or mi } \\
\text { MBTR or MBSR or M } \\
\text { choeducation or } \mathrm{ACT} \\
\text { GCBT):AB,EH,KW,KY } \\
\text { AND CENTRAL:TARG }\end{array}$ & $\begin{array}{l}\text { 7. ((cogniti* } \\
\text { or accep } \\
\text { mitment } \\
\text { adj6 (the } \\
\text { havior }^{\star} \text { o }\end{array}$ & $\begin{array}{l}\text { ance } \\
\text { or } \\
6 \\
\text { av- } \\
\text { ur }^{\star} \text { or }\end{array}$ \\
\hline $\begin{array}{l}\# 7 \text { ((cogniti* or relaxation or accep- } \\
\text { tance or commitment or adaptation) } \\
\text { near (therap* or behavior* or behaviour* } \\
\text { or strateg }^{*} \text { or intervention* or ap- } \\
\text { proach or psychotherap* or training } \\
\text { or treatment or technique }{ }^{*} \text { or program* } \\
\text { or counselling or counselling)):AB,E- } \\
\text { H,KW,KY,MC,MH,TI,TO AND CEN- } \\
\text { TRAL:TARGET }\end{array}$ & $\begin{array}{l}\text { or strateg* or inter- } \\
\text { vention* or approach* } \\
\text { or psychotherap* or } \\
\text { training or treatment } \\
\text { or technique* or pro- } \\
\text { gram or counselling }^{*} \text { or counselling)).ab,ti. } \\
\text { 8. ((behaviour* or behav- }\end{array}$ & $\begin{array}{l}\text { strateg* or interven- } \\
\text { tion* or approach* }^{*} \\
\text { or psychotherap* or } \\
\text { training or treatment } \\
\text { or technique* or pro- } \\
\text { gram* or counselling } \\
\text { or counselling)).ab,ti. } \\
\text { 8. ((behaviour or be- }\end{array}$ \\
\hline $\begin{array}{l}\text { \#8 ((behaviour* or behavior }{ }^{\star} \text { or medi- } \\
\text { tation) near (strateg* or intervention }{ }^{\star} \text { or } \\
\text { therap* or approach* or psychotherap* } \\
\text { or technique* or counselling or coun- } \\
\text { selling)):AB,EH,KW,KY,MC,MH,TI,TO } \\
\text { AND CENTRAL:TARGET }\end{array}$ & $\begin{array}{l}\text { (strateg* or interven- } \\
\text { tion* or therap* or }^{*} \\
\text { approach }^{\star} \text { or psycho- } \\
\text { therap* or technique } \\
\text { or counselling or }\end{array}$ & $\begin{array}{l}\text { havior* or meditation) } \\
\text { adj6 (strateg* or inter- } \\
\text { vention* or therap* or } \\
\text { approach* or psycho- }^{*} \\
\text { therap* or technique }^{\star} \\
\text { or counselling or }\end{array}$ \\
\hline \#9 \# & & \\
\hline & 9. 1 or 2 & 9. 1 or 2 \\
\hline \#3 & & 10.3 or $4 c$ \\
\hline & 11.9 and 10 & 11.9 and 10 \\
\hline
\end{tabular}

Web of Science 
Chapter 3

5 Search strategies. Continued.

\begin{tabular}{|c|c|c|}
\hline \#1 TOPIC: (tinnit*) & S11 S9 AND S10 & \multirow{17}{*}{$\begin{array}{l}\text { CT.gov } \\
\text { Condition: tinnitus } \\
\text { Study Type: Interventional }\end{array}$} \\
\hline Indexes=SCI-EXPANDED, SSCI, CP- & S10 S7 OR S8 & \\
\hline & S9 S1 OR S2 OR S3 OR S4 & \\
\hline \#2 TOPIC: (CBT or ACT or mindful- & OR S5 OR S6 & \\
\hline $\begin{array}{l}\text { ness or MBTR or MBSR or MBTSR or } \\
\text { psychoeducation or IACT or iCBT or }\end{array}$ & S8 TX tinnit* & \\
\hline GCBT) & S7 (MH “tinnitus") & \\
\hline $\begin{array}{l}\text { Indexes=SCI-EXPANDED, SSCI, CP- } \\
\mathrm{Cl}-\mathrm{S}, \mathrm{CPCl}-\mathrm{SSH} \text { Timespan=All years }\end{array}$ & $\begin{array}{l}\text { S6 TX (behaviour }{ }^{\star} \text { or } \\
\text { behavior }^{\star} \text { or meditation) n6 }\end{array}$ & \\
\hline $\begin{array}{l}\text { \#3 TOPIC: ((cogniti* or relaxation or } \\
\text { acceptance or commitment or adapta- } \\
\text { tion) near/6 (therap* or behavior* or be- } \\
\text { haviour* or strateg* or intervention* or }\end{array}$ & $\begin{array}{l}\text { (strateg* }^{*} \text { intervention* or } \\
\text { therap* or approach* or psy- } \\
\text { chotherap* or technique }^{*} \text { or } \\
\text { counseling or counselling) }\end{array}$ & \\
\hline $\begin{array}{l}\text { approach }^{*} \text { or psychotherap* or training } \\
\text { or treatment or technique }{ }^{\star} \text { or program* } \\
\text { or counselling or counselling)) }\end{array}$ & $\begin{array}{l}\text { S5 TX (cogniti* or relax- } \\
\text { ation or acceptance or } \\
\text { commitment or adaptation) }\end{array}$ & \\
\hline $\begin{array}{l}\text { Indexes=SCI-EXPANDED, SSCI, CP- } \\
\text { CI-S, CPCI-SSH Timespan=All years }\end{array}$ & $\begin{array}{l}\text { n6 } \text { (therap* or behavior* or }^{*} \\
\text { behaviour }^{*} \text { or strateg* or } \\
\text { intervention* or approach }\end{array}$ & \\
\hline $\begin{array}{l}\text { \#4 TOPIC: ((behaviour }{ }^{\star} \text { or behavior } \text { br }^{\star} \\
\text { meditation) near/6 (strateg }{ }^{\star} \text { or interven- } \\
\text { tion }^{\star} \text { or therap* or approach }{ }^{\star} \text { or psy- } \\
\text { chotherap* or technique }^{*} \text { or counselling }\end{array}$ & $\begin{array}{l}\text { or psychotherap* or training } \\
\text { or treatment or technique* } \\
\text { or program* or counseling } \\
\text { or counselling) }\end{array}$ & \\
\hline or counselling)) & S4 TX CBT or ACT or mind- & \\
\hline $\begin{array}{l}\text { Indexes=SCI-EXPANDED, SSCI, CP- } \\
\text { Cl-S, CPCl-SSH Timespan=All years }\end{array}$ & & \\
\hline \#5 \#4 OR \#3 OR \#2 & $\begin{array}{l}\text { cation or } \mathrm{AACT} \text { or iCBT or } \\
\text { GCBT }\end{array}$ & \\
\hline $\begin{array}{l}\text { Indexes=SCI-EXPANDED, SSCI, CP- } \\
\mathrm{Cl}-\mathrm{S}, \mathrm{CPCl}-\mathrm{SSH} \text { Timespan=All years }\end{array}$ & S3 (MH "Meditation+") & \\
\hline \#6 \#5 AND \#1 & $\begin{array}{l}\text { S2 (MH "Behavior Thera- } \\
\text { py+") }\end{array}$ & \\
\hline $\begin{array}{l}\text { Indexes=SCI-EXPANDED, SSCI, CP- } \\
\mathrm{Cl}-\mathrm{S}, \mathrm{CPCl}-\mathrm{SSH} \text { Timespan=All years }\end{array}$ & $\begin{array}{l}\text { S1 (MH "Adaptation, Psy- } \\
\text { chological+") }\end{array}$ & \\
\hline
\end{tabular}


Meta-analysis of CBT for tinnitus 



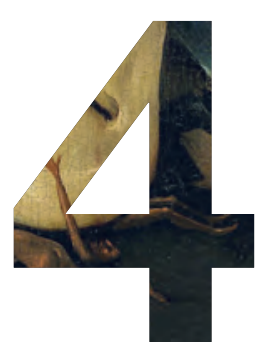

\section{THE FEAR OF TINNITUS}

\section{QUESTIONNAIRE: TOWARD A}

\section{VALID AND RELIABLE MEANS OF}

\section{ASSESSING FEAR IN ADULTS}

\section{WITH TINNITUS}

Published as: Fuller, T. E., Cima, R. F. F., Van den Bussche, E., \& Vlaeyen, J. W. S. (2019). The Fear of Tinnitus Questionnaire: Toward a Reliable and Valid Means of Assessing Fear in Adults with Tinnitus. 


\section{Abstract}

Objective: The purpose of this study was to assess the factor structure, reliability and validity of the Fear of Tinnitus Questionnaire (FTQ); a brief self-report measure of people's fears about potential cognitive, emotional, behavioral and social consequences of living with tinnitus.

Design: Five hundred and eighty-eight Dutch-speaking adults with tinnitus completed an online battery of questionnaires measuring tinnitus related distress, fear, catastrophizing and quality of life. The sample was randomly split in two in order to perform exploratory and Bayesian confirmatory factor analyses. A subsample of participants $(\mathrm{N}=144)$ completed the battery of questionnaires a second time after a two week interval in order to calculate test-retest reliability and conduct a Bland-Altman analysis. Convergent and concurrent validity of the FTQ was assessed with the complete data set and measures of tinnitus related distress as the outcome.

Results: Exploratory factor analyses indicated that a single and three-factor FTQ model were both valid solutions. Posterior predictive $p$ values for the Bayesian confirmatory factor analyses ranged between .51 and .53 indicating that the respective models were an excellent fit for the data. The FTQ showed excellent test-retest reliability (average value ICC $=.92 ; 95 \% \mathrm{Cl}, 0.89-0.95$ ) and in the Bland-Altman analysis, satisfactory agreement between participants' scores after a two week interval. Furthermore the FTQ demonstrated good internal reliability $(a=.83,95 \% \mathrm{Cl}[.81-.85])$ and added statistically significant amounts of variance to models predicting tinnitus related distress and interference in daily life.

Conclusions: The FTQ has good psychometric properties and can be used to assess people's fear of tinnitus in research or clinical settings. Further work to establish the reliability and validity should be conducted and include an examination of a version of the FTQ that uses Likert-type response scales which might offer improved sensitivity.

Keywords: tinnitus, fear, tinnitus, psychometric, validity, reliability 


\section{Introduction}

Subjective tinnitus is defined as the perception of sound in the absence of an observable or objectively measureable internal or external source. Approximately $15-20 \%$ of the adult population reports tinnitus (Davis \& El Refaie, 2000; Kim et al., 2015) which can be considered to have two main elements - the perceived sound and the reaction to it (Hoare, Edmondson-Jones, Gander, \& Hall, 2014). Subjective tinnitus (hereafter simply referred to as tinnitus) is frequently, but not always, associated with hearing loss and other otological conditions such as hyperacusis and otosclerosis (Langguth, Kreuzer, Kleinjung, \& De Ridder, 2013). It is as of yet unclear how acute tinnitus develops into chronic debilitating complaints. While many people experience tinnitus only a small proportion (i.e. 1-3\%; Davis \& El Refaie, 2000; Fujii et al., 2011; Kim et al., 2015) report severe levels of distress, interference in social and occupational functioning, difficulties with concentration, memory dysfunction, sleep problems, lower quality of life, and severe psychological suffering (Andersson \& Edvinsson, 2008; Joo, Han, \& Park, 2015; Tyler \& Baker, 1983). The psychological consequences associated with living with bothersome tinnitus include increased chances of higher levels of depression and anxiety symptoms (Langguth, 2011; Pattyn et al., 2015; Pinto et al., 2014; M. D. Sullivan et al., 1988; Zöger, Svedlund, \& Holgers, 2006), and lower self-esteem (Krog, Engdahl, \& Tambs, 2010). These potential undesirable consequences combined with troubling thoughts about possible physical or psychological damage in all likelihood add to the conviction in many patients that tinnitus is something to fear.

Fear is an emotional state or response triggered by existing or imminent threats to one's health or safety. It is usually considered adaptive as it motivates people to try to minimise or prevent harm from occurring. However, persistent efforts to avoid or escape (perceived) threatening situations where (increased) tinnitus is expected can be considered maladaptive. That is, in the long term the level of distress and disability might worsen as a consequence, for example, of people avoiding or withdrawing from social or work situations that they fear might worsen the tinnitus.

\section{Fear avoidance model of tinnitus}

The fear avoidance model (FA) has, as its name suggests, the concepts of fear and avoidance as its central tenets. It is a cognitive behavioural model that was developed in an attempt to better understand and predict the distress and interference in mood and daily life activities of people living with chronic pain (Lethem, Slade, Troup, \& Bentley, 1983; Vlaeyen, Crombez, \& Linton, 2016; Vlaeyen \& Linton, 2000, 2012). The similarities between chronic pain and chronic tinnitus (Møller, 1997, 2000) have proved informative for the understanding of tinnitus suffering and hence the FA model has recently been applied to tinnitus (Cima, Crombez, \& Vlaeyen, 2011; Kleinstäuber et al., 2013). [See Cima, Crombez, et al. (2011) or Kleinstäuber et al. (2013) for a visual representation of the FA model of tinnitus.] The FA model of tinnitus is predictive in that it suggests directional relationships between the respective variables within it. The model offers the possibility to formulate hypotheses about how the perception of tinnitus triggers cognitive, emotional and behavioural responses leading to distress and interference 
in some people but not others. Specifically, it predicts that through catastrophic (mis) interpretations about the sound such as that it is a sign of impending harm (Andersson \& McKenna, 2006), the subsequent fear of the tinnitus leads to behavioural strategies to avoid instances of worsened tinnitus (Budd \& Pugh, 1996; Hallberg, Erlandsson, \& Carlsson, 1992). The model also predicts that people who interpret the tinnitus sound as benign will not have fearful reactions, and therefore will not engage in avoidant, escape or safety behaviours.

\section{Development of and previous research with the Fear of Tinnitus Questionnaire} Tinnitus-related fear as a concept has thus far received relatively little attention in the research literature and was not specifically measured until the Fear of Tinnitus Questionnaire (FTQ; Chapter 4 Supplementary Information File) was developed. It is a brief self-report measure that specifically aims to measure levels of fear of experiencing tinnitus and possible consequences associated with it. The FTQ was based on two measures of fear associated with pain [the Tampa scale for Kinesiophobia (TSK; Roelofs et al., 2007) and the Pain Anxiety Symptoms Scale (PASS; McCracken, Zayfert, \& Gross, 1992)], was designed to produce a total score indicative of fear of tinnitus, and to be used as an outcome measure rather than a diagnostic tool. Higher scores on the FTQ are intended to indicate higher levels of tinnitus-related fear.

Recent research in a clinical sample has found that high levels of fear of tinnitus are indeed associated with higher tinnitus-distress and poorer quality of life (Cima, Crombez, et al., 2011; Cima, van Breukelen, \& Vlaeyen, 2017). Comprehensive mediation analyses indicated that reductions in tinnitus related fear explain the improvements in quality of life and decrease in tinnitus related distress following specialised CBT for tinnitus (Cima et al., 2017).

Additionally in a large randomised controlled trial, results showed that the FTQ total score changed over time and between two clinical treatment groups (Cima et al., 2012). While the FTQ total score appears to work as intended, researchers have not previously examined its psychometric properties. Further, we do not know if the FTQ is actually a unifactorial measure, has redundant items, or has subscales that explain/ predict tinnitus-related distress. The purpose of this study was thus to investigate the psychometric properties of the FTQ by examining the validity and reliability of the factor structure and the ability of the measure to predict levels of tinnitus related distress. It extends existing research examining data from the FTQ (e.g.Cima, Crombez, et al., 2011) by using data from a sample of people experiencing tinnitus but not participating in a clinical trial. No hypotheses were made regarding the test-retest reliability or the number of underlying factors likely to be identified in the FTQ. We expected that the FTQ total score would positively correlate with health anxiety, illness behaviour, and physical and mental health. Finally, based on the FA model (Lethem et al., 1983; Vlaeyen et al., 2016; Vlaeyen \& Linton, 2000, 2012), fear of tinnitus, as measured by the FTQ, was predicted to be associated with measures of tinnitus related disability, distress and intensity. 


\section{Materials and Methods}

A brief overview of the participants and procedures are provided here as they have been described in detail in a previous publication (Cima, Crombez, et al., 2011). Ethics approval to conduct the study was given by the Ethics Committee of the Faculty of Psychology and Neuroscience at Maastricht University, the Netherlands (approval number: 72_04_07_2008).

\section{Participants}

Advertisements placed on the websites of the Dutch Association for Hearing Disorders (www.nvvs.nl) and the Dutch Tinnitus Platform (www.tinnitus.nl) between November 2008 and April 2009 invited visitors to the website to participate in a large crosssectional study that included a battery of tinnitus-specific, general health, and mood related measures. Participants who experienced, but were not necessarily bothered or distressed by, tinnitus were eligible to participate in this study. We have chosen to select a broad range of participants to maximize generalizability. We included a broad variety of tinnitus sufferers, including variety concerning their experienced level of distress.

Seven-hundred and ninety-one respondents requested additional information about the study and consent forms. Of the 615 participants who returned their consent form and completed at least one questionnaire, 588 completed the FTQ. For the purposes of this study, data from the 588 participants who completed the Fear of Tinnitus questionnaire were retained for analysis. That is, there were no missing data on the FTQ, but potentially on other questionnaires (see Table 5). (Note: not all participants provided response to questions about age, gender, tinnitus duration and other demographic variables and thus the sample size numbers do not always equal 588.) The sample included 230 women with a mean age of 50 years (range 18-80 years; SD 11.33 years) and 342 men with a mean age of 53.5 years (range 17-81 years; SD 53.46). Most of the participants were employed and approximately $80 \%$ were not currently receiving any treatment for tinnitus. Table 1 and 2 report demographic characteristics for the samples used for exploratory and confirmatory factor analysis respectively.

\section{Procedure}

After giving consent, participants received unique login codes via email to access the online assessment by questionnaires. The measures used in this study were selected because of their sound psychometric properties, relevance for the research question and frequent use in tinnitus research as outcome measures. The battery of questionnaires which were all presented in Dutch was hosted on the 'Emium'website (www.emium.nl; Janssen, 2008). The order of questionnaires and questions was fixed, with questionnaires being presented as a self-contained set of questions with specific instructions and clear demarcations of the start and end points. The questionnaires were presented in the following order: Short Form-36, Tinnitus Catastrophizing Scale, Tinnitus Questionnaire, Illness Attitudes Scale, Fear of Tinnitus Questionnaire, Tinnitus Disability Index, demographic questions and visual analogue scales measuring tinnitus intensity. Participants were able to read and complete them independently but were not 
required to complete the questionnaires in one sitting (although they were instructed to complete them within a day), and hence could login and out as they wished.

For the purpose of establishing the test-retest reliability of the FTQ, a subset of 250 participants were invited exactly two weeks after first completing the questionnaire battery to do so a second time. Over half $(\mathrm{N}=144,58 \%)$ of these participants who were not undertaking treatment for tinnitus related distress (according to self-report) completed all the measures in the questionnaire battery a second time.

The complete sample $(\mathrm{N}=588)$ was randomly split precisely in half in order to perform the exploratory and confirmatory factor analyses on two separate sub-samples. We chose to split the sample in half for cross validation purposes in accordance with the recommendations of Gerbing and Hamilton (1996). They found - by using Monte Carlo methods - that EFA contributes to model specification prior to cross-validation using CFA and was hence a useful method in identifying a model that could be evaluated with CFA.

\section{Measures}

The Cronbach alpha's for the questionnaires listed below are presented in Table 3 with values shown for the respective samples.

Fear of Tinnitus Questionnaire (FTQ; Cima, Crombez, et al., 2011). The FTQ is a 17item self-report measure designed to assess respondents' level of fear regarding their tinnitus. Items in the questionnaire are presented as a series of statements (e.g. "I am afraid that my tinnitus will become worse") for which respondents are asked to indicate if it is applicable to their current situation. Each statement receives a score of 1 when applicable and 0 when not applicable. The maximum is 17 with higher scores reflecting higher levels of tinnitus related fear. The total score is the sum of all applicable statements and provides an overall rating of fear of tinnitus. The FTQ has shown to be sensitive to change following treatment (Cima et al., 2012).

Tinnitus Catastrophizing Scale (TCS; Cima, Crombez, et al., 2011) assessed thinking the worst about tinnitus (i.e. catastrophizing). The TCS is a 13-item measure based on the Pain Catastrophizing Scale (M. J. L. Sullivan, Bishop, \& Pivik, 1995). Respondents use a five-point scale ranging from 0 to 4 to indicate the degree to which statements (e.g. "It's terrible and I think it's never going to get any better") applies to them. Higher scores indicate higher levels of catastrophizing and with the maximum being 62.

The Tinnitus Questionnaire (TQ; Hallam, Jakes, \& Hinchcliffe, 1988; Meeus, Blaivie, \& Van de Heyning, 2007) is a widely used measure of tinnitus related distress. It contains 52 items, has a three-point scale representing "true" "partly true" and "not true" that respondents use to indicate levels of agreement with the respective items (e.g., "It's unfair that I have to suffer with my noises") and a maximum score of 84 (minimum is zero). Higher scores indicate greater levels of tinnitus-related distress. The TQ has six subscales within the measure that includes emotional and cognitive distress, sleep disturbance, intrusiveness, auditory and perceptual difficulties and somatic complaints attributable to the tinnitus. The TQ has high internal consistency, convergent and 
discriminant validity and is sensitive to change (Baguley, Humphriss, \& Hodgson, 2000; Zeman, Koller, Schecklmann, Langguth, \& Landgrebe, 2012).

The Tinnitus Disability Index (TDI; Cima, Vlaeyen, Maes, Joore, \& Anteunis, 2011) was used to measure tinnitus related functional impairment. The TDI is a 7-item measure that uses an 11 point Likert-type scale (range from 0 to 10; maximum score 70) to assess the degree to which respondents' daily life activities (e.g., social activities) are adversely affected by tinnitus. Higher scores indicate higher levels of disability. The respective anchors are: "no disability" and "total disability". The TDI has sound psychometric properties and is strongly correlated with measures of quality of life and psychological distress associated with tinnitus (Cima, Vlaeyen, et al., 2011).

Three questions were used to measure the intensity of participants' tinnitus. [Note that, at the time of data collection, the Tinnitus Magnitude Index (Schmidt et al., 2014) had not been developed yet.] The respective questions, ("How do you judge your tinnitus when it was at its worst? How do you judge your tinnitus when it was at its least? How did you judge your tinnitus when it was at its usual intensity?") had to be answered using visual analogue scales with the endpoint anchors "not intense at all" and "the most intense imaginable". Participants' were instructed to "click" on a point on the respective horizontal lines that was indicative of the tinnitus severity. Although it was not visible to respondents, the points along the horizontal line represented scores from 0 to 100 . Although it is not usual practice to do so, scores for each question were averaged to produce a single "tinnitus intensity" score to be used in the subsequent analyses.

The IIIness attitudes scale (IAS; Kellner, 1987) is a 29-item measure of fears, beliefs and attitudes related to health. The original version includes nine subscales although a factor analysis of the Dutch version indicated that a two-factor solution provided the best model fit. The two scales - health anxiety (items 2-4, 6, 13-17, 19, and 21) and illness behaviour (items 23-25 and 27-29) (Speckens, Spinhoven, Sloekers, Bolk, \& van Hemert, 1996) are used in this study. The IAS asks respondents to report on a five-point Likert-type scale the frequency with which they experience particular health related thoughts or behaviours. The response options range from "no" (zero points) to "most of the time" (four points) with higher scores indicating poorer greater levels of health anxiety and illness behaviour respectively. The maximum scores for Health Anxiety and IIIness Behaviour subscales are 48 and 24 respectively. The IAS has been demonstrated to possess stable test-retest reliability of the subscales (Fava, Kellner, Zielezny, \& Grandi, 1988; Kellner, 1987) and has good concurrent, convergent and discriminant validity (Stewart \& Watt, 2000).

The Short Form-36 (SF-36; Hays, Sherbourne, \& Mazel, 1993; Mosges, Koberlein, Erdtracht, \& Klingel, 2008; Ware Jr et al., 1998) is a widely used self-report measure of health. It has 36 items that can form two independent global scores that relate to physical and mental health respectively (McHorney, Ware, \& Raczek, 1993). Higher scores (maximum equals 100 and minimum equals 0 ) indicate better quality of life. The SF-36 has been translated into Dutch, validated and is considered appropriate for use with people with or without a chronic health condition (Aaronson et al., 1998). 
Although an SF-36 total score is sometimes calculated, it is not recommended by the developers of the tool (Lins \& Carvalho, 2016), and hence we use the two summary health scores.

Demographics. Participants responded to a brief set of demographic questions asking for details of their gender, age, education, duration of tinnitus, hearing loss, current treatments, employment status, and history of absences from work due to illness.

\section{Analysis}

R version 3.2 and the "userfriendlyscience" package (Peters, 2014) were used to calculate confidence intervals and estimates of reliability for the questionnaires. IBM SPSS Statistics version 24 was used for exploratory factor analysis, to calculate correlations and hierarchical regressions to inform judgements about concurrent and convergent validity and test-retest reliability. We used IBM SPSS AMOS version 24 to conduct Bayesian confirmatory factor analysis. In all analyses where data was missing, pairwise deletion was used instead of imputation methods.

We adopted a conservative approach in testing the assumption that that FTQ is best interpreted as a single-factor measure.

Data used in the analyses are held in a repository managed by Maastricht University and available upon request.

Exploratory factor analysis. The FTQ items have binary response options, in which case it is not advised to use traditional factor analytic methods (Lee \& Song, 2003) as it can lead to "biased standard errors and significance tests, overestimation of the number of factors and underestimation of the factor loadings" (Woods, 2002). Given this, tetrachoric correlation matrices were used instead and were calculated using a macro specifically developed for the purpose by Lorenzo-Seva and Ferrando (2012) in IBM SPSS Statistics version 24 . The assumptions and conditions regarding the suitability of the dataset were examined prior to conducting analyses. Specifically, the ratio of sample size to number of variables, Kaiser-Meyer-Olkin measure of sampling adequacy and Bartlett's test of sphericity were checked. Principal Axis Factoring (PAF) with oblique rotation was conducted on the tetrachoric correlation matrices. The number of factors to extract was determined by examining the scree plot, Kaiser's criterion (i.e. eigenvalues $>1$ ) and parallel analysis. Parallel analysis (Horn, 1965) is an accurate and robust (Zwick \& Velicer, 1986) Monte Carlo simulation technique that estimates the number of possible factors to be retained. The simulations/calculations are based on random data sets that have the same number of variables and sample size as the real correlation matrix being analysed. Factor loadings of .32 or greater were considered sufficient (Comrey \& Lee, 1992). However, we did not automatically discard items with loadings that were below, but still close to, this cut-off value since it was possible that items could be theoretically or conceptually similar to other items on a subscale.

Confirmatory factor analysis. To conduct CFA for dichotomous data, IBM SPSS AMOS version 24 offers Bayesian CFA. This method has several advantages over a frequentist 
approach. For example, it can provide good performance in small samples; avoid inadmissible model parameter values; offer computational advantages and intuitive interpretation of the results, as well as allow for the specification of background knowledge through the specification of prior knowledge of parameters/distributions (Hoofs, van de Schoot, Jansen, \& Kant, 2017). We did not however specify a prior distribution, since with a large sample size selecting priors makes little difference to outcomes and as a result used the Amos default uniform distribution setting (Arbuckle, 2013). A cut off was set at 1.1 for convergence of the model (Hu \& Bentler, 1999) and posterior predictive $p$ was used for assessing goodness of fit of model. Posterior predictive $p$ is a Bayesian estimate of goodness of fit where the closer the posterior predictive $p$ values are to .50 the better the model fit is considered (Gelman, 2013; Muthen \& Asparouhov, 2012). ${ }^{1}$

Test-retest reliability. A subset of 144 respondents not undertaking treatment completed the questionnaires again after a 2-week interval for the purposes of collecting data for test-retest analysis. We used two methods to assess test-retest reliability. First, the intraclass correlation coefficient using a two-way mixed model with absolute agreement was calculated. Second, we conducted a Bland-Altman analysis (Bland \& Altman, 1986) to examine the level of agreement between the FTQ total scores at the respective time points. The Bland-Altman analysis is comprised of three steps: first, a single sample $t$-test to determine if the mean difference between the scores at the two time points was significantly different from zero (from a statistical perspective); second, plotting these differences between the two FTQ total scores by the average FTQ total scores across the two time points (see Figure 1); and finally, conducting a simple linear regression to examine if proportional bias was present.

Convergent validity was investigated by examining the correlations between the total score of the FTQ with measures of tinnitus related distress and impairment (i.e. TQ and TDI), the health anxiety and illness behaviour scales of the IAS, and the physical and mental health summary measure scores of the SF-36.

In order to test concurrent validity (the relationship between a distinct but related concept/measure) of the FTQ, we examined the unique contribution of tinnitus related fear in accounting for variability in tinnitus-distress (i.e. TQ) and tinnitus related disability (i.e. TDI) using hierarchical regression. The criterion for multicolinearity - variance inflation factor (VIF) - was set at 2. The FTQ total scores were entered into the regression analyses after controlling for age, gender, education, self-reported tinnitus severity, health anxiety and illness behaviour, and mental and physical health as measured by the SF- 36 .

\footnotetext{
${ }^{1}$ For further discussion of posterior predictive $p$ values see Meng (1994) and Rubin (1984).
} 


\section{Results}

\section{Sample equivalence}

Chi-square and independent samples $t$-tests using IBM SPSS statistics version 24 revealed that the samples used for the exploratory and confirmatory factor analyses were equivalent. There were no significant differences on gender, highest completed level of education, age, duration of tinnitus, whether they had hearing loss, the severity of the tinnitus, the location of their tinnitus, employment status and whether they were currently receiving treatment (Table 1 and Table 2).

Table 1. Participant Characteristics and P-values from Chi-Square Tests Indicating EFA and CFA Sample Equivalence

\begin{tabular}{llllll}
\hline \multirow{2}{*}{ Characteristic } & & \multicolumn{4}{c}{ Sample } \\
& & $\begin{array}{l}\text { EFA } \\
\mathrm{n}(\%)\end{array}$ & $\begin{array}{l}\text { CFA } \\
\mathrm{n}(\%)\end{array}$ & $\begin{array}{l}\text { Total } \\
\mathrm{n}(\%)\end{array}$ & $\mathrm{p}$ \\
\hline Gender & Female & $118(41.69)$ & $112(38.75)$ & $230(40.20)$ & .47 \\
& Male & $165(58.31)$ & $177(61.25)$ & $342(59.80)$ & \\
Education & Primary & $33(11.66)$ & $39(13.49)$ & $72(12.59)$ & .52 \\
& Secondary & $66(23.32)$ & $63(21.80)$ & $129(22.55)$ & \\
& Vocational training & $55(19.43)$ & $62(21.45)$ & $117(20.45)$ & \\
Location of tinnitus & Left ear & $66(23.32)$ & $58(20.07)$ & $124(21.67)$ & .39 \\
& Higher education & $129(45.56)$ & $125(43.25)$ & $254(44.40)$ & \\
& Right ear & $34(12.01)$ & $48(16.60)$ & $82(14.34)$ & \\
Hearing loss & Both ears & $146(51.59)$ & $143(49.48)$ & $289(50.52)$ & \\
Employed & Middle of head & $37(13.07)$ & $40(13.84)$ & $77(13.46)$ & \\
& Present & $140(49.46)$ & $135(46.71)$ & $275(48.07)$ & .51 \\
Currently receiving & Yes & $174(30.42)$ & $179(31.29)$ & $353(61.71)$ & .49 \\
treatment & Yos & $115(20.10)$ & $104(18.18)$ & $219(38.29)$ & \\
\hline
\end{tabular}

Note. $\mathrm{EFA}=$ Exploratory factor analysis; CFA = Confirmatory factor analysis. 
Table 2. Descriptive Statistics of Participants' Tinnitus and Age by Sample with P-Values from Independent Samples T-Test Indicating Equivalence between Groups

\begin{tabular}{llllll}
\hline \multirow{2}{*}{ Characteristic } & & \multicolumn{4}{c}{ Sample } \\
& & EFA & CFA & Total & $p$ \\
& & $\mathrm{~N}=283$ & $\mathrm{~N}=289$ & & .17 \\
\hline Tinnitus duration & Years & 5.42 & 5.59 & 5.51 & .24 \\
& $(S D)$ & $(1.49)$ & $(1.43)$ & $(1.46)$ & \\
Tinnitus severity & VAS & 5.59 & 5.82 & 5.71 & .24 \\
& $(S D)$ & $(2.00)$ & $(1.97)$ & $(1.99)$ & \\
Age & Years & 51.30 & 52.84 & 52.07 & .11 \\
& $(S D)$ & $(12.07)$ & $(11.14)$ & $(11.63)$ & \\
\hline
\end{tabular}

Note. EFA= Exploratory factor analysis; CFA = Confirmatory factor analysis; VAS= Visual analogue scale. Missing age related data for $n=16$ participants

Table 3. Internal Consistency for Questionnaires Used by Respective Samples.

\begin{tabular}{lllll}
\hline \multirow{2}{*}{ Measure } & \multicolumn{4}{c}{ Sample $a(95 \% \mathrm{Cl})$} \\
& $\begin{array}{l}\text { Entire sample } \\
(\mathrm{N}=588)\end{array}$ & $\begin{array}{l}\text { Test-retest } \\
(\mathrm{n}=144)\end{array}$ & $\begin{array}{l}\text { EFA } \\
(\mathrm{n}=294)\end{array}$ & $\begin{array}{l}\text { CFA } \\
(\mathrm{n}=294)\end{array}$ \\
\hline FTQ & $.83(.81-.85)$ & $.84(.80-.88)$ & $.82(.79-.85)$, & $.84,(.81-.86)$ \\
TCS & $.93(.93-.94)$ & $.94(.93-.95)$ & $.94(.92-.95)$ & $.93(.92-.94)$ \\
TQ & $.94(.93-.95)$ & $.95(.94-0.96)$ & $.94(.93-.95)$ & $.94(.93-.95)$ \\
TDI & $.89(.87-.90)$ & $.89(.86-.92)$ & $.90(.88-.92)$ & $.87(.85-.90)$ \\
IAS & $.85(.83-.86)$ & $.88(.86-.91)$ & $.85(.83-.87)$ & $.85(.82-.87)$ \\
SF-36 PH & $.91(.90-.92)$ & $.91(.89-.92)$ & $.92(.91-.92)$ & $.90(.89-.92)$ \\
SF-36 MH & $.93(.92-.93)$ & $.93(.92-.95)$ & $.93(.91-.94)$ & $.93(.91-.94)$ \\
\hline
\end{tabular}

Note. $\mathrm{EFA}=$ Exploratory factor analysis; $\mathrm{CFA}=$ Confirmatory factor analysis; FTQ = Fear of Tinnitus Questionnaire; IAS = IIIness Attitude Scale; SF-36 PH = Short Form-36 Physical Health; SF-36 $\mathrm{MH}=$ Short Form 36 Mental Health; TCS = Tinnitus Catastrophizing Scale; TDI = Tinnitus Disability Index; TQ = Tinnitus Questionnaire.

\section{Exploratory factor analysis}

Exploratory factor analysis was conducted using Principal Axis Factoring (PAF) on a random selection of half the participants $(n=294)$ on the FTQ with oblique rotation. The Kaiser-Meyer-Olkin (KMO) measure of sampling adequacy was poor (KMO = .121) indicating that some items might need to be removed from the analysis. Bartlett's test of Sphericity $X^{2}(136)=4289.05, p<.001$, however, was sufficient indicating that the correlations between items were large enough to proceed with factor analysis. There was variation between the pre-specified methods for determining how many factors to extract. Specifically, six factors had eigenvalues larger than 1; the scree plot (Chapter 4 Supplementary Information File, p. 2) indicated that retaining up to four factors could be appropriate and parallel analysis indicated that three factors would be optimal. Given 
this variation, we examined four possible factor structures, with one to four retained factors, in attempt to identify the most parsimonious solution.

A single factor solution accounted for $30.6 \%$ of the variance, while two, three or four factor solutions accounted for $43.3 \%, 54.9 \%$ and $62.3 \%$ of the variance respectively. Inspection of the two-factor pattern matrix (Chapter 4 Supplementary Information File, $p$. 3) revealed that the first factor contained 11 items and the second contained four items. Item 12 did not load on either factor (factor loadings of .18 and -.01 respectively) while item 16 loaded on both factors (.50 and .31 respectively). This type of cross loadings is undesirable (Costello \& Osborne, 2005). Based on this, we considered removing items 12 and 16. Item 6 showed a loading of .28 which we considered to be close to the cut-off. Additionally, as this item did not have cross loadings, we decided to retain it in the analysis. However, this item should be treated with some caution. The threefactor pattern matrix (Table 4) yielded three factors with eight, four and three items respectively. Item 12 did not load on any factor and item 16 loaded on two factors, which indicates that they do not appear to be measuring the same construct, and thus could be removed from further analysis. Again, item 6 had a loading of .28 but showed no cross loadings and was conceptually similar to other items on the subscale. In the three-factor solution, the third factor comprised the three items that showed the lowest loadings on the factor with 11 items in the two-factor solution. A four-factor solution (Chapter 4 Supplementary Information File, p. 4) yielded two factors with four items, one factor with three and one factor with only one item respectively. Four items (8, 13, 14 and 16) loaded on two factors. Item 12 did not load on any of the possible four factors.

Table 4. Pattern Matrix of Exploratory Factor Analysis - Three Factor Solution for the Fear of Tinnitus Questionnaire $(\mathrm{N}=294)$

Factor

\begin{tabular}{|c|c|c|c|}
\hline Item number and statement & $\begin{array}{l}\text { Future } \\
\text { consequences }\end{array}$ & Somatic & Deterioration \\
\hline $\begin{array}{l}\text { (5) I am afraid that my tinnitus will drive me } \\
\text { crazy }\end{array}$ & .796 & .154 & -.022 \\
\hline $\begin{array}{l}\text { (8) I am afraid the moment will come that my } \\
\text { head cannot withstand tinnitus anymore }\end{array}$ & .595 & .153 & .148 \\
\hline $\begin{array}{l}\text { ( } 9 \text { ) My mental condition will become severely } \\
\text { affected by my tinnitus }\end{array}$ & .731 & .066 & -.026 \\
\hline $\begin{array}{l}\text { (10) I am afraid that tinnitus will stop me from } \\
\text { ever having a normal life again }\end{array}$ & .696 & -.076 & -.090 \\
\hline $\begin{array}{l}\text { (11) I am afraid that I will never be able to experi- } \\
\text { ence silence again because of tinnitus }\end{array}$ & .701 & -.141 & .049 \\
\hline $\begin{array}{l}\text { (13) I am afraid I will not be able to do anything } \\
\text { anymore because of my tinnitus }\end{array}$ & .503 & .110 & .113 \\
\hline $\begin{array}{l}\text { (14) It worries me to think I may never be able to } \\
\text { learn how to cope with this condition }\end{array}$ & .860 & -.053 & -.090 \\
\hline
\end{tabular}


Table 4. Continued.

\begin{tabular}{|c|c|c|c|}
\hline \multirow[b]{2}{*}{ Item number and statement } & \multicolumn{3}{|c|}{ Factor } \\
\hline & $\begin{array}{l}\text { Future conse- } \\
\text { quences }\end{array}$ & Somatic & Deterioration \\
\hline $\begin{array}{l}\text { (15) It would be terrible if my tinnitus proved a } \\
\text { life-long condition }\end{array}$ & .434 & -.090 & .142 \\
\hline $\begin{array}{l}\text { (16) I am concerned that tinnitus may be a risk } \\
\text { to my physical health }\end{array}$ & .505 & .312 & .009 \\
\hline (3) I fear that my tinnitus is the result of a tumour & -.089 & .876 & .037 \\
\hline $\begin{array}{l}\text { (4) Even though my tinnitus is getting worse. I do } \\
\text { not think it points to a serious disease }\end{array}$ & -.142 & .729 & -.021 \\
\hline $\begin{array}{l}\text { (17) I am afraid that tinnitus may be a preliminary } \\
\text { sign of brain haemorrhage or similar }\end{array}$ & .160 & .579 & .150 \\
\hline $\begin{array}{l}\text { (6) The fact that I have tinnitus does not mean } \\
\text { that my health is at risk }\end{array}$ & .105 & .282 & -.058 \\
\hline $\begin{array}{l}\text { (1) I am afraid that my tinnitus will deteriorate my } \\
\text { hearing }\end{array}$ & -.137 & .005 & .986 \\
\hline $\begin{array}{l}\text { (2) I am afraid that my tinnitus will become } \\
\text { worse }\end{array}$ & .131 & -.066 & .452 \\
\hline (7) I am afraid my tinnitus will leave me deaf & -.077 & .108 & .890 \\
\hline $\begin{array}{l}\text { (12) I am afraid that loud noises will aggravate } \\
\text { my tinnitus }\end{array}$ & .102 & $\underline{-.013}$ & .149 \\
\hline Eigen values & 5.20 & 2.17 & 1.96 \\
\hline$\%$ of variance & 30.57 & 12.75 & 11.55 \\
\hline a & .80 & .53 & .67 \\
\hline$(95 \% \mathrm{Cl})$ & $.77-.84$ & $.45-.61$ & $.60-.73$ \\
\hline
\end{tabular}

Note. Bold indicates factor loadings above or close to the cut-off of.32. Underlined values indicate cross loading of items on two or more factors.

Of these possible solutions, overall, it appeared that the single factor and three-factor solutions were more defensible than the two or four-factor solutions. First, the four-factor solution was clearly less coherent with a factor containing only a single item and four items showed cross loadings. Second, the three-factor solution actually groups the items with lowest loadings from the two-factor solution neatly to form a meaningful third factor. A single factor solution accounts for a little over $30 \%$ of the variance and is most consistent with the intention of the developers of the FTQ. On the other hand, a three factor solution accounts for a greater proportion of variance (55\%), and has clusters of items that could theoretically represent meaningful subscales of fears relating to: future consequences (items: 5, 8, 9, 10, 11, 13, 14, 15); hearing and tinnitus related deterioration (items: 1, 2, 7); and, somatic conditions (items: 3, 4, 6, 17). The internal consistency of these potential FTQ subscales was $a=.80(95 \% \mathrm{Cl}, 0.77-0.84)$ for future consequence, $a=.67(95 \% \mathrm{Cl}, 0.60-0.73)$ for hearing and tinnitus related deterioration, and $a=.53$ $(95 \% \mathrm{Cl}, 0.45-0.61)$ for somatic related fears. 


\section{Confirmatory Factor Analyses}

Given the results of the exploratory factor analysis three models were tested: two single factor models (one model with and one model without items 12 and 16) and a three factor model (items 12 and 16 removed) with the subscales of future consequences, deterioration and somatic related fears, using the confirmatory sample $(n=294)$.

Since the FTQ items have binary response options, the asymptotic distribution function (ADF) was used, as this does not require the assumption of normality to be met. Bayesian estimation was conducted to generate estimates of the goodness of fit (posterior predictive $p$ values) for the respective models. The posterior predictive $p$ values for the respective models for the single factor solution were: FTQ-17 item $=.53$, FTQ-15 item $=.52$ and for the three-factor solution: FTQ-15 item $=.51$. Detailed results from the Bayesian analysis showing the regression weights, intercepts and variance are provided in the Chapter 4 Supplementary Information File, pages 5-16. The posterior predictive $p$ values indicate that each of the respective models provides an excellent fit with the data from the confirmatory sample. No other indices of goodness of fit were calculated due to the binary nature of the data.

The following results all relate to the 17-item version of the FTQ.

\section{Test-retest reliability}

The average value ICC for the FTQ total score was excellent (.92; 95\% Cl, $0.89-0.95)$ and statistically significant $[F(144,144)=13.82, p<.001]$ indicating that respondents' total FTQ scores were very similar when completed after a two-week interval.

The Bland-Altman analysis revealed a more a slightly more complex picture in the data over time. Specifically, the single sample $t$-test indicated that the mean difference $(0.56$ between the scores) was significantly different from zero $[t(144)=3.46$. $p<.01)]$ which suggests that there is limited agreement between the FTQ scores at the respective time points. However, from a clinical perspective, an average difference of less than one is unlikely to be meaningful. Subsequent simple linear regression analysis predicting the difference between the FTQ total scores based on the average FTQ total score indicated that there was no proportional bias in the data $[F(1,143)=0.05, p>.05$, with an $\left.\mathrm{R}^{2}=.00\right]$. This means that there was no systematic variation in the FTQ total scores at time one and two over the range of possible scores. For example, the differences in total FTQ scores between time point one and two did not increase with increasing average FTQ total score.

The Bland-Altman plot (Figure 1) reveals that eight of the 144 participants' results were outside the upper and lower limits of agreement (defined as the mean difference of the scores at time points one and two $\pm 1.96 S D$ of the differences). Overall, this result suggests that there was satisfactory agreement between participants' scores after a two-week interval. 
Figure 1. Bland-Altman Plot for FTQ Total Score at Time Points 1 and $2(\mathrm{~N}=144)$

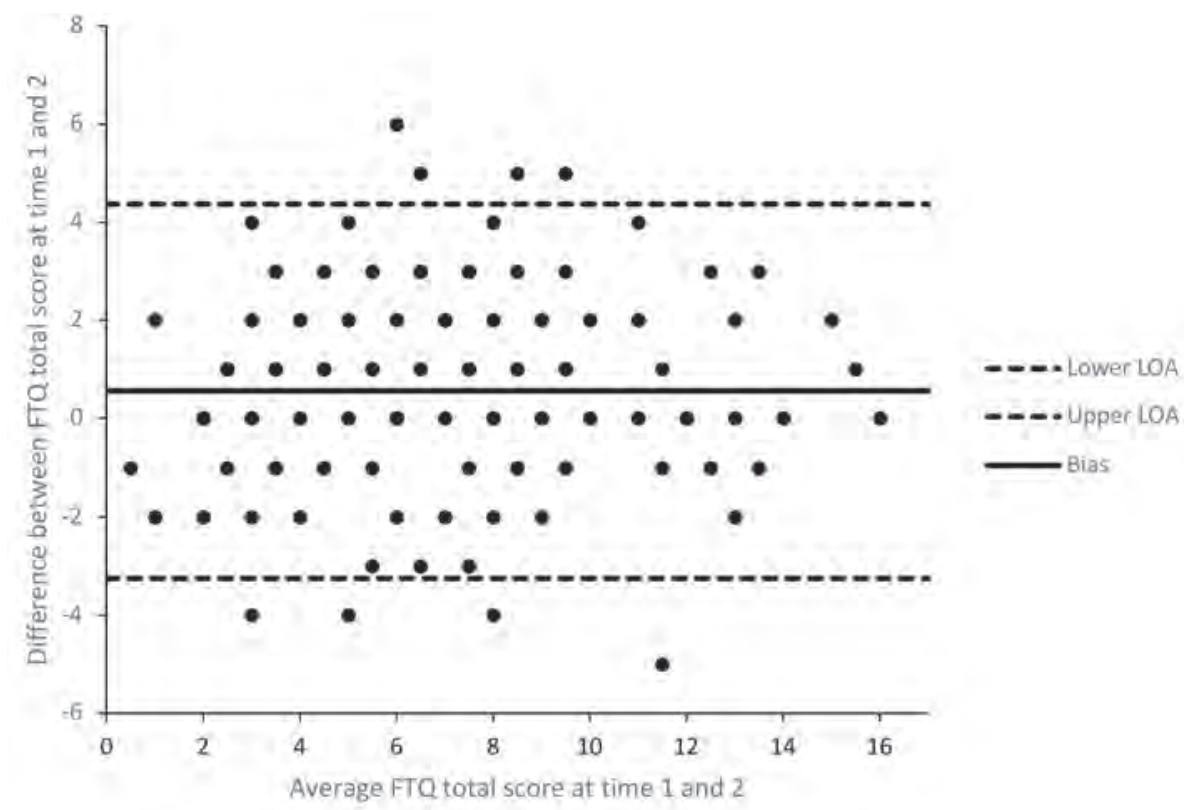

Note: LOA: Limits of agreement defined as the mean difference of the scores at time points one and two $\pm 1.96 S D$ of the differences.

\section{Convergent validity}

Table 5 and 6 respectively show the descriptive statistics and bivariate correlations for the FTQ, and target variables tinnitus disability (TDI), tinnitus intensity, health anxiety, illness behaviour, overall physical and mental health and tinnitus related distress (TQ). Table 6 reveals that virtually all the variables are positively and significantly correlated with each other but that the strength of association varies. Specifically, the FTQ total score is strongly correlated (i.e. $r \geq .7$ ) with the TCS and TQ total scores, moderately correlated (i.e. $r=.3$ to .7) with TDI, SF-36 MH, IAS-HA, IAS-IB and weakly (i.e. $r \leq .3$ ) correlated with SF-36 PH. These results suggest that someone who is more afraid of tinnitus (i.e. high scores on the FTQ) is more likely to also experience higher levels of: (a) tinnitus related distress; (b) catastrophizing; (c) health anxiety; (d) tinnitus-interference in daily life; (e) illness behaviour; (f) tinnitus intensity; (g) mental health problems; (h) physical health problems. 
Chapter 4

Table 5. Descriptive Statistics from Outcome Measures

\begin{tabular}{lllll}
\hline Measure & Mean $(95 \% \mathrm{Cl})$ & $S D$ & Min-Max & $\mathrm{N}$ \\
\hline FTQ & $4.66(4.44-4.88)$ & 2.76 & $0-12$ & 588 \\
TCS & $21.75(20.8-22.71)$ & 11.78 & $0-52$ & 588 \\
TDI & $248(235-261)$ & 154 & $0-648$ & 581 \\
TQ & $47.09(45.57-48.62)$ & 18.83 & $5-96$ & 588 \\
SF-36 PH & $45.97(45.10-46.85)$ & 10.77 & $17-79$ & 588 \\
SF-36 MH & $45.39(44 / 48-46.31)$ & 11.26 & $23-78$ & 588 \\
IAS-HA & $9.43(8.89-9.97)$ & 6.67 & $0-36$ & 588 \\
IAS-IB & $11.97(11.60-12.35)$ & 4.59 & $3-26$ & 588 \\
Tinnitus Intensity & $5.71(5.51-5.91)$ & 1.99 & $0-10$ & 385 \\
\hline
\end{tabular}

Note. FTQ = Fear of Tinnitus Questionnaire; $\mathrm{TCS}=$ Tinnitus Catastrophizing Scale; $\mathrm{TDI}=$ Tinnitus Disability Index; SF-36 PH = Short Form-36 Physical Health; SF-36 MH = Short Form-36 Mental Health; TQ = Tinnitus Questionnaire; IAS-HA = IIIness Attitude Scale-Health Anxiety; IAS-IB = IIIness Attitude Scale-IIIness Behaviour; Tinnitus Intensity = average tinnitus intensity

\section{Concurrent validity}

A series of four hierarchical regressions (see Tables 7 and Table 8) were conducted to examine the respective proportions of variance explained in the TDI, TQ, SF36$\mathrm{PH}$ and SF36-MH by the FTQ total score. In each regression, age, gender, education and average tinnitus intensity were the first group of variables entered into the model. The second block of variables entered into the model included mental and physical health, health anxiety, and illness behaviour except in the instance when SF36-PH and $\mathrm{MH}$ respectively were used as the dependent variable. Multicollinearity was a problem between the FTQ and TCS (VIF = 2.64). Given that, the regression equations were run omitting TCS from the models. The FTQ total score significantly contributes to explaining variance in tinnitus related distress and impairment as measured by the TQ and TDI respectively (Table 7). The model however accounted for less variance of TDI as a dependent variable [adjusted $\left.R^{2}=.43[F(9,375)=33.72, p<.001)\right]$ compared with TQ as a dependent variable [adjusted $\left.R^{2}=.70[F(9,375)=102.07, p<.001)\right]$ indicating that the FTQ total score is better suited for explaining distress rather than tinnitus related disability. The models also predict a larger proportion of variance in mental health [adjusted $\left.R^{2}=.31[F(8,376)=22.72, p<.001)\right]$ compared with physical health [adjusted $\left.R^{2}=.25[F(8,376)=17.37, p<.001)\right]$ as measured by the SF-36 (Table 8). 
Table 6. Pearson's Correlation Coefficients of The Fear of Tinnitus Questionnaire Total Score with Included Measures

\begin{tabular}{|c|c|c|c|c|c|c|c|c|}
\hline Measure & FTQ & TCS & TDI & $\mathrm{TQ}$ & $\begin{array}{l}\text { SF-36 } \\
\mathrm{PH}\end{array}$ & $\begin{array}{l}\text { SF-36 } \\
\mathrm{MH}\end{array}$ & IAS-HA & IAS-IB \\
\hline TCS & $.72^{*}$ & & & & & & & \\
\hline TDI & $.53^{*}$ & $.51^{*}$ & & & & & & \\
\hline TQ & $.75^{\star}$ & $.77^{*}$ & $.71^{*}$ & & & & & \\
\hline SF-36 PH & $.16^{\star}$ & $.16^{*}$ & $.33^{*}$ & $.25^{*}$ & & & & \\
\hline SF-36 MH & $.40^{*}$ & $.38^{*}$ & $.43^{*}$ & $.48^{*}$ & $.41^{*}$ & & & \\
\hline IAS-HA & $.43^{*}$ & $.36^{*}$ & $.21^{*}$ & $.29^{*}$ & .03 & $.24^{*}$ & & \\
\hline IAS-IB & $.33^{*}$ & $.28^{*}$ & $.48^{*}$ & $.45^{*}$ & $.45^{\star}$ & $.43^{*}$ & $.24^{*}$ & \\
\hline Tinnitus intensity & $.45^{\star}$ & $.51^{*}$ & $.48^{*}$ & $.59^{*}$ & $.20^{*}$ & $.29^{*}$ & $.15^{*}$ & $.34^{*}$ \\
\hline
\end{tabular}

Note. There was no missing data on all questionnaires/scores except for TDI $(n=581)$ and Tinnitus Intensity $(n=385)$. We used pairwise deletion rather than imputation of values for missing data. FTQ = Fear of Tinnitus Questionnaire; TCS = Tinnitus Catastrophizing Scale; TDI = Tinnitus Disability Index; SF-36 PH = Short Form-36 Physical health; SF-36 MH = Short-Form-36 Mental health; $\mathrm{TQ}=$ Tinnitus Questionnaire; IAS=HA = IIIness Attitude Scale-Health Anxiety; IAS-IB = IIIness Attitude Scale-IIIness Behaviour; Tinnitus Intensity = average tinnitus intensity ${ }^{*} \mathrm{p}<.01$

Table 7. Summary of Hierarchical Regression Analysis for Predicting Tinnitus Related Disability and Distress

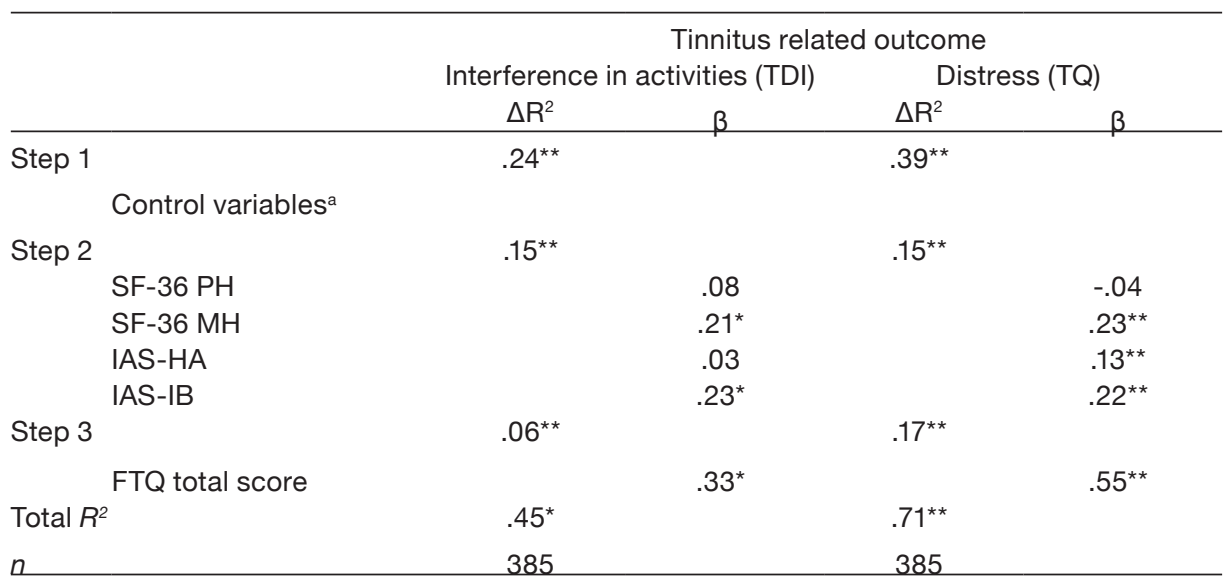

Note: a Control variables included age, gender, education, average tinnitus intensity.

$\Delta \mathrm{R}^{2}=$ change in amount of variance explained at each step in the regression model. $\beta$ values indicate how much the Tinnitus Questionnaire and Tinnitus Disability Index scores change by when the FTQ total score changes by one standard deviation. FTQ = Fear of Tinnitus Questionnaire; IAS-HA = IIIness Attitude Scale-Health Anxiety; IAS-IB = IIIness Attitude Scale-IIIness Behaviour; SF-36 PH = Short Form-36 Physical Health; SF-36 MH = Short Form 36 Mental Health; TDI = Tinnitus Disability Index; TQ = Tinnitus Questionnaire.

${ }^{*} \mathrm{p}<.01^{* *} \mathrm{p}<.001$. 
Chapter 4

Table 8. Summary of Hierarchical Regression Analysis for Variables Predicting Mental and Physical Health (i.e. SF-36 Subscales)

\begin{tabular}{|c|c|c|c|c|}
\hline & \multicolumn{4}{|c|}{ Health outcome } \\
\hline & \multicolumn{2}{|c|}{ Mental Health } & \multicolumn{2}{|c|}{ Physical Health } \\
\hline & $\Delta \mathrm{R}^{2}$ & $\beta$ & $\Delta \mathrm{R}^{2}$ & $\beta$ \\
\hline Step 1 & $.09^{\star \star}$ & & $.07^{\star \star}$ & \\
\hline \multicolumn{5}{|c|}{ Control variables ${ }^{a}$} \\
\hline Step 2 & $.20^{\star \star}$ & & $.20^{\star *}$ & \\
\hline & & $.26^{\star}$ & & (NA) \\
\hline & & (NA) & & $.27^{*}$ \\
\hline & & $.15^{\star}$ & & $-.13^{*}$ \\
\hline & & $.26^{\star}$ & & $.32^{*}$ \\
\hline Step 3 & $.03^{\star \star}$ & & .00 & \\
\hline & & $.23^{*}$ & & -.09 \\
\hline Total $R^{2}$ & $.33^{\star \star}$ & & $.27^{\star \star}$ & \\
\hline$n$ & 385 & & 385 & \\
\hline
\end{tabular}

Note: ${ }^{\text {a }}$ Control variables included age, gender, education, average tinnitus intensity.

$\Delta R^{2}$ = change in amount of variance explained at each step in the regression model. $\beta$ values indicate how much the SF-36 Mental health and SF-36 Physical health scores respectively change by, when the FTQ total score is changes by one standard deviation. FTQ = Fear of Tinnitus Questionnaire; IAS-HA = IIIness Attitude Scale-Health Anxiety; IAS-IB = IIIness Attitude Scale-IIIness Behaviour; SF-36 PH = Short Form-36 Physical Health; SF-36 MH = Short Form 36 Mental Health; $\mathrm{TDI}=$ Tinnitus Disability Index; TQ = Tinnitus Questionnaire.

${ }^{*} \mathrm{p}<.01{ }^{* *} \mathrm{p}<.001$

\section{Response distributions}

A post-hoc, exploratory examination of the response frequency on the FTQ items revealed that five statements (numbers 3, 4, 6, 13 and 17) had poor discriminatory power. Approximately $85 \%$ of participants indicated that items 4, 6 and 13, and $94 \%$ indicated items 3 and 17 did not apply to their current situation.

\section{Discussion}

The main purpose of this study was to examine the reliability, validity and factor structure of the Fear of Tinnitus Questionnaire. One- and three-factor solutions were assessed as providing a good fit for the dataset. The single factor solution accounted for approximately $30 \%$ of variance, compared with $55 \%$ of the variance for the three-factor solution. The internal consistency of the single factor solution was excellent, whereas the internal consistency of the three-factor solution was variable, with all items of one subscale (somatic - items $3,4,6,17$ ) having poor internal consistency and discriminatory power (i.e. most participants indicated that these items did not apply to their current situation). 
There were similarities though in the themes of the subscales identified in the PASS (McCracken et al., 1992; Roelofs et al., 2004) and TSK (Roelofs et al., 2007). For example, the somatic subscale in the three-factor model of the FTQ has similar items to the somatic focus subscale in the TSK (Roelofs et al., 2007). Results indicated that the FTQ (17-items) total score gives stable results over a two-week period (i.e. has good test-retest reliability) and contributes significantly to models predicting tinnitus related distress as measured by the TQ and tinnitus related impairment as measured by the TDI.

Assessing the convergent validity of the FTQ was, at the time of data collection, made difficult by the absence of alternative measures of tinnitus related fear. In lieu of that, the IAS-Health Anxiety subscale was used to provide an indication of convergent validity. The moderate correlation might result from two key differences between the FTQ and IAS. Specifically the FTQ aims to measure fear of a specific, present or imminent threat to health (i.e. tinnitus) whereas the IAS-Health Anxiety subscale assesses levels of worry regarding possible aversive future health concerns that are more general in nature, or potentially even not relevant to respondents. In contrast, strong correlations were found between the FTQ and the TQ, the TCS and the TDI. The TQ, which is a measure of global tinnitus related distress, does not purport to measure tinnitus related fear specifically. The strong correlation with the TQ could be due to many items on the FTQ relating to consequences of having tinnitus - albeit with a focus on being afraid of rather than actually experiencing them which is in accordance with the FA model. The strong correlation between the TCS and FTQ total scores is consistent with the FA model, which predicts that higher levels of catastrophizing results in higher levels of fear. The relatively weak correlations between the FTQ total score and measures of physical health and illness behaviour are also logically consistent (although not explicitly predicted a priori) as a high level of fear of an outcome or condition does not necessarily mean that one already has poor physical health and/or that one acts in a sick role.

To our knowledge, the FTQ is the first questionnaire designed to solely measure tinnitus related fear. The Tinnitus Fear Avoidance Cognitions and Behaviours Scale (T-FAS; Kleinstäuber et al., 2013) assesses tinnitus fear related cognitions and avoidance behaviours. It includes a subscale - cognitions - that contains items that might have a strong conceptual overlap to those found on the FTQ. For example both measures include items regarding the fear that tinnitus will adversely affect quality of life. In their study examining the properties of the T-FAS, Kleinstäuber and colleagues found, with a sample of participants seeking treatment, that the cognitions subscale was moderately $(r=.57)$ correlated with the Tinnitus Handicap Inventory total score and with measures of anxiety $(r=.46)$ and anxiety sensitivity $(r=.45)$. The pattern of results reported here is consistent with these results even though the samples differ (that is, a web-based compared with treatment seeking sample).

\section{Limitations}

There are several potential limitations of the current study that need to be considered. Firstly, the data was collected from questionnaires presented in a fixed order online that might have attracted a specific sample of participants. In addition to there being potential order effects and participants being self-selected, participants did not undergo any examination 
or assessment of hearing loss, tinnitus or verification of other characteristics and could have completed the questionnaires over a period of days even though they were instructed to complete it within a single a day. If respondents' tinnitus fluctuated over time, this might have influenced how they responded to the measures. While these issues might limit the degree to which the findings can be generalised to other samples, participants would have had little to gain by exaggerating or minimising their symptoms or experience with tinnitus. The sample actually shares some similarities with that reported by Kleinstäuber et al. (2013) (e.g., the ratio of female participants, and the average age and education level of participants was nearly identical). The samples differ though on the proportion of participants reporting hearing loss (approximately $48 \%$ in this sample compared with $75 \%$ ) and duration of living with tinnitus (five compared with eight) years.

Although it is not necessarily a methodological limitation of this study, it is possible that the format of the response options (dichotomous) of the FTQ affected the measure's ability to distinguish between respondents with varying degrees of fear of tinnitus that in turn affects the measure's concurrent (predictive) validity. That is a Likert-type scale offers respondents greater choice in how much fear they might report for a specific item rather than simply indicating whether they experience any fear. Debate exists in the literature regarding the optimal number of response options to survey questions and the impact that less compared with more options has on results (Clark \& Watson, 1995). Some have argued that there are extensive problems (e.g. relating to accuracy of response choice, and reliability) with dichotomous response options that affect validity and discriminating power (Comrey, 1988; Preston \& Colman, 2000). Others have argued that reliability and test-retest reliability in particular, are not affected by dichotomous data (Matell \& Jacoby, 1971; Preston \& Colman, 2000). In this case, the dichotomous response options did not affect test-retest reliability.

Given that approximately $90 \%$ of participants indicated that five of the 17 items were irrelevant to them, possibly reflects that participants already had a good level of knowledge about the aetiology of tinnitus. This seems possible given that they had, on average experienced tinnitus for over five years and were visiting websites where information about tinnitus was available. Additionally, participants might suffered only a mild-to-moderate level of tinnitus fear. By way of comparison, after extensive CBT treatment, participants had an average FTQ total score of 4.2012 months after baseline measurements (Cima et al., 2012) compared with participants in this study who had an average FTQ score of 4.66. Similarly, Swedish patients in a recent online cohort study had a mean total FTQ score of 4.00 (Müller et al., 2016). Regardless, the FTQ total score has been shown to be a variable that has explanatory power in predicting/detecting treatment response in a clinical trial of stepped care specialised CBT for tinnitus (Cima et al., 2012).

Lastly, it is noted that we did not predict, a priori, the strength of association between measures and that there is a high level of intercorrelation between the FTQ and the TQ. This high level of intercorrelation is likely to be because there is, in part, conceptual overlap between the FTQ and TQ. The TQ includes several subscales (e.g. emotional distress, somatic complaints) which produce a global assessment of tinnitus-distress severity. In line with this, a recent mediation analysis demonstrated how reductions in tinnitus 
related fear (as measured with the FTQ) partially explain why specialised CBT is effective in improving quality of life and reducing tinnitus related distress (Cima et al., 2017).

\section{Future directions}

Given these issues with the FTQ it might be informative for future research to examine the effect of changing response options to a 5-point Likert-type scale on the factor structure and ability to contribute to models predicting tinnitus related distress or impairment respectively. (A study addressing this is currently in progress.) It would also be informative to examine

- the effect of the mode of completing the FTQ (e.g. online compared with by pen and paper) as there is conflicting research about whether it matters or not (Bowling, 2005; van de Looij-Jansen \& de Wilde, 2008; Walt, Atwood, \& Mann, 2008; Wang, Liu, Cheng, \& Cheng, 2013),

- how the T-FAS and FTQ relate in samples of people with tinnitus seeking and not seeking treatment respectively,

- validate the FTQ in different populations of people with tinnitus who might interpret their tinnitus differently from those in the sample recruited for this study, and

- the reliability and validity of a 15-item version of the FTQ.

In order to assess face validity it would also be of interest to examine what people with tinnitus think of the actual measure. Do they, for example, actually think in terms of being afraid or fearful of particular consequences or risks associated with their tinnitus and or hearing loss? Additionally, examining and/or revising the wording of item 6 would be useful, as this item showed a rather weak factor loading and low relevance to participants' current situation. A qualitative approach similar to one used to examine the subscales of the Tampa Scale of Kinesiophobia (Bunzli, Smith, Watkins, Schutze, \& O’Sullivan, 2015) might yield valuable information in this regard.

Finally, future research could specifically examine the somatic subscale items to investigate their relevance for individuals with tinnitus considering the duration of the complaints. People with recent onset tinnitus might be relatively naïve about it and find the items on the somatic sub-scale to be applicable. In contrast, those who have experienced tinnitus for a longer period may be reasonably well informed about the aetiology and possible implications of tinnitus, and thus are aware that most of the items are unlikely to be true. Additionally, the effects of excluding item 6 from the FTQ based on its low factor loading on the psychometric properties need further study.

\section{Conclusion}

The results of this study indicate that the Dutch version of the FTQ has good psychometric properties and that for tinnitus research purposes the FTQ total score is a valid and informative outcome measure. The three-factor version might be useful for clinicians, since it provides specific information on fear-related thoughts that could subsequently form part of the focus during treatment. 


\section{References}

Aaronson, N. K., Muller, M., Cohen, P. D., Essink-Bot, M. L., Fekkes, M., Sanderman, R., . . Verrips, E. (1998). Translation, validation, and norming of the Dutch language version of the SF-36 Health Survey in community and chronic disease populations. J Clin Epidemiol, 51(11), 1055-1068. doi:10.1016/S0895-4356(98)00097-3

Andersson, G., \& Edvinsson, E. (2008). Mixed feelings about living with tinnitus: A qualitative study. Audiological Medicine, 6(1), 48-54. doi:doi:10.1080/16513860801899355

Andersson, G., \& McKenna, L. (2006). The role of cognition in tinnitus. Acta Otolaryngologica, 126(s556), 39-43. doi:doi:10.1080/03655230600895226

Arbuckle, J. L. (2013). IBM AMOS SPSS 22 User's Guide. Chicago, IL.: SPSS Inc.

Baguley, D. M., Humphriss, R. L., \& Hodgson, C. A. (2000). Convergent validity of the tinnitus handicap inventory and the tinnitus questionnaire. J Laryngol Otol, 114(11), 840-843.

Bland, J. M., \& Altman, D. G. (1986). Statistical methods for assessing agreement between two methods of clinical measurement. Lancet, 1(8476), 307-310.

Bowling, A. (2005). Mode of questionnaire administration can have serious effects on data quality. Journal of Public Health, 27(3), 281-291. doi:10.1093/pubmed/fdi031

Budd, R. J., \& Pugh, R. (1996). Tinnitus coping style and its relationship to tinnitus severity and emotional distress. J Psychosom Res, 41(4), 327-335. doi:10.1016/ S0022-3999(96)00171-7

Bunzli, S., Smith, A., Watkins, R., Schutze, R., \& O'Sullivan, P. (2015). What Do People Who Score Highly on the Tampa Scale of Kinesiophobia Really Believe?: A Mixed Methods Investigation in People With Chronic Nonspecific Low Back Pain. Clin J Pain, 31(7), 621-632. doi:10.1097/ajp.0000000000000143

Cima, R. F. F., Crombez, G., \& Vlaeyen, J. W. (2011). Catastrophizing and fear of tinnitus predict quality of life in patients with chronic tinnitus. Ear Hear, 32(5), 634-641. doi:10.1097/AUD.0b013e31821106dd

Cima, R. F. F., Maes, I. H., Joore, M. A., Scheyen, D. J., El Refaie, A., Baguley, D. M., . . . Vlaeyen, J. W. (2012). Specialised treatment based on cognitive behaviour therapy versus usual care for tinnitus: a randomised controlled trial. Lancet, 379(9830), 19511959. doi:10.1016/s0140-6736(12)60469-3

Cima, R. F. F., van Breukelen, G., \& Vlaeyen, J. W. S. (2017). Tinnitus-related fear: Mediating the effects of a cognitive behavioural specialised tinnitus treatment. Hearing Research, in press. doi:10.1016/j.heares.2017.10.003

Cima, R. F. F., Vlaeyen, J. W., Maes, I. H., Joore, M. A., \& Anteunis, L. J. (2011). Tinnitus interferes with daily life activities: a psychometric examination of the Tinnitus Disability Index. Ear Hear, 32(5), 623-633. doi:10.1097/AUD.0b013e31820dd411 
Clark, L. A., \& Watson, D. (1995). Constructing validity: Basic issues in objective scale development. Psychological Assessment, 7(3), 309-319. doi:10.1037/1040-3590.7.3.309

Comrey, A. L. (1988). Factor-analytic methods of scale development in personality and clinical psychology. J Consult Clin Psychol, 56(5), 754-761. doi:10.1037/0022006X.56.5.754

Comrey, A. L., \& Lee, H. B. (1992). A first course in factor analysis. (2nd ed.). Hillsdale, New Jersey: Lawrence Erlbaum Associates.

Costello, A. B., \& Osborne, J. W. (2005). Best Practices in Exploratory Factor Analysis: Four Recommendations for Getting the Most From Your Analysis. Practical Assessment, Research \& Evaluation, 10(7), epub 1 - 9.

Davis, A., \& El Refaie, A. (2000). Epidemiology of Tinnitus. In R. S. Tyler (Ed.), Handbook of Tinnitus (pp. 1-24). San Diego: Singular thompson Learning.

Fava, G. A., Kellner, R., Zielezny, M., \& Grandi, S. (1988). Hypochondriacal fears and beliefs in agoraphobia. Journal of Affective Disorders, 14(3), 239-244. doi:10.1016/0165-0327(88)90040-7

Fujii, K., Nagata, C., Nakamura, K., Kawachi, T., Takatsuka, N., Oba, S., \& Shimizu, H. (2011). Prevalence of tinnitus in community-dwelling Japanese adults. J Epidemiol, 21(4), 299-304.

Gelman, A. (2013). Two simple examples for understanding posterior p-values whose distributions are far from unform. Electronic Journal of Statistics, 7, 2595-2602. doi:10.1214/13-EJS854

Gerbing, D. W., \& Hamilton, J. G. (1996). Viability of exploratory factor analysis as a precursor to confirmatory factor analysis. Structural Equation Modeling: $A$ Multidisciplinary Journal, 3(1), 62-72. doi:10.1080/10705519609540030

Hallam, R. S., Jakes, S. C., \& Hinchcliffe, R. (1988). Cognitive variables in tinnitus annoyance. Br J Clin Psychol, 27(3), 213-222. doi:10.1111/j.2044-8260.1988.tb00778.x

Hallberg, L. R. M., Erlandsson, S. I., \& Carlsson, S. G. (1992). Coping strategies used by middle-aged males with noise-induced hearing loss, with and without tinnitus. Psychology \& Health, 7(4), 273-288. doi:10.1080/08870449208403157

Hays, R. D., Sherbourne, C. D., \& Mazel, R. M. (1993). The rand 36-item health survey 1.0. Health Economics, 2(3), 217-227. doi:10.1002/hec. 4730020305

Hoare, D. J., Edmondson-Jones, M., Gander, P. E., \& Hall, D. A. (2014). Agreement and Reliability of Tinnitus Loudness Matching and Pitch Likeness Rating. PLoS ONE, 9(12), e114553. doi:10.1371/journal.pone.0114553

Hoofs, H., van de Schoot, R., Jansen, N. W. H., \& Kant, I. (2017). Evaluating Model Fit in Bayesian Confirmatory Factor Analysis With Large Samples: Simulation Study Introducing the BRMSEA. Educational and Psychological Measurement, 78(4), 537568. doi:10.1177/0013164417709314 
Horn, J. L. (1965). A rationale and test for the number of factors in factor analysis. Psychometrika, 30(2), 179-185. doi:10.1007/bf02289447

Hu, L., \& Bentler, P. M. (1999). Cutoff criteria for fit indexes in covariance structure analysis: Conventional criteria versus new alternatives. Structural Equation Modeling: A Multidisciplinary Journal, 6(1), 1-55. doi:10.1080/10705519909540118

Janssen, R. (2008). EMIUM: Effect Monitoring via Internet Universiteit Maastricht (Version 1.3): Maastricht: Research Institute of Experimental Psychopathology, Maastricht University.

Joo, Y. H., Han, K. D., \& Park, K. H. (2015). Association of Hearing Loss and Tinnitus with Health-Related Quality of Life: The Korea National Health and Nutrition Examination Survey. PLoS ONE, 10(6), e0131247. doi:10.1371/journal.pone.0131247

Kellner, R. (1987). Abridged manual of the IIIness Attitudes Scale: Department of Psychiatry, School of Medicine, University of New Mexico.

Kim, H. J., Lee, H. J., An, S. Y., Sim, S., Park, B., Kim, S. W., . . Choi, H. G. (2015). Analysis of the prevalence and associated risk factors of tinnitus in adults. PLoS ONE, 10(5), e0127578. doi:10.1371/journal.pone.0127578

Kleinstäuber, M., Jasper, K., Schweda, I., Hiller, W., Andersson, G., \& Weise, C. (2013). The role of fear-avoidance cognitions and behaviors in patients with chronic tinnitus. Cogn Behav Ther, 42(2), 84-99. doi:10.1080/16506073.2012.717301

Krog, N. H., Engdahl, B., \& Tambs, K. (2010). The association between tinnitus and mental health in a general population sample: Results from the HUNT Study. $J$ Psychosom Res, 69(3), 289-298. doi:10.1016/j.jpsychores.2010.03.008

Langguth, B. (2011). A review of tinnitus symptoms beyond 'ringing in the ears': a call to action. Curr Med Res Opin, 27(8), 1635-1643. doi:10.1185/03007995.2011.595781

Langguth, B., Kreuzer, P. M., Kleinjung, T., \& De Ridder, D. (2013). Tinnitus: causes and clinical management. Lancet Neurol, 12(9), 920-930. doi:10.1016/s14744422(13)70160-1

Lee, S.-Y., \& Song, X.-Y. (2003). Bayesian analysis of structural equation models with dichotomous variables. Stat Med, 22(19), 3073-3088. doi:10.1002/sim.1544

Lethem, J., Slade, P. D., Troup, J. D., \& Bentley, G. (1983). Outline of a Fear-Avoidance Model of exaggerated pain perception-I. Behav Res Ther, 21(4), 401-408. doi:10.1016/0005-7967(83)90009-8

Lins, L., \& Carvalho, F. M. (2016). SF-36 total score as a single measure of healthrelated quality of life: Scoping review. SAGE Open Medicine, 4, 2050312116671725. doi:10.1177/2050312116671725

Lorenzo-Seva, U., \& Ferrando, P. J. (2012). TETRA-COM: a comprehensive SPSS program for estimating the tetrachoric correlation. Behav Res Methods, 44(4), 11911196. doi:10.3758/s13428-012-0200-6 
Matell, M. S., \& Jacoby, J. (1971). Is There an Optimal Number of Alternatives for Likert Scale Items? Study I: Reliability and Validity. Educational and Psychological Measurement, 31(3), 657-674. doi:10.1177/001316447103100307

McCracken, L. M., Zayfert, C., \& Gross, R. T. (1992). The Pain Anxiety Symptoms Scale: development and validation of a scale to measure fear of pain. Pain, 50(1), 67-73. doi:10.1016/0304-3959(92)90113-P

McHorney, C. A., Ware, J. E., Jr., \& Raczek, A. E. (1993). The MOS 36-Item Short-Form Health Survey (SF-36): II. Psychometric and clinical tests of validity in measuring physical and mental health constructs. Med Care, 31(3), 247-263.

Meeus, O., Blaivie, C., \& Van de Heyning, P. (2007). Validation of the Dutch and the French version of the Tinnitus Questionnaire. B-ENT, 3 Suppl 7, 11-17.

Meng, X.-L. (1994). Posterior Predictive p-Values. Ann. Statist., 22(3), 1142-1160. doi:10.1214/aos/1176325622

Møller, A. R. (1997). Similarities between chronic pain and tinnitus. Am J Otol, 18(5), 577-585.

Møller, A. R. (2000). Similarities between severe tinnitus and chronic pain. J Am Acad Audiol, 11(3), 115-124.

Mosges, R., Koberlein, J., Erdtracht, B., \& Klingel, R. (2008). Quality of life in patients with idiopathic sudden hearing loss: comparison of different therapies using the Medical Outcome Short Form (36) Health Survey questionnaire. Otol Neurotol, 29(6), 769-775. doi:10.1097/MAO.0b013e31817e5d03

Müller, K., Edvall, N. K., Idrizbegovic, E., Huhn, R., Cima, R., Persson, V., . . . Cederroth, C. R. (2016). Validation of Online Versions of Tinnitus Questionnaires Translated into Swedish. Frontiers in Aging Neuroscience, 8, 272. doi:10.3389/fnagi.2016.00272

Muthen, B., \& Asparouhov, T. (2012). Bayesian structural equation modeling: a more flexible representation of substantive theory. Psychol Methods, 17(3), 313-335. doi:10.1037/a0026802

Pattyn, T., Van Den Eede, F., Vanneste, S., Cassiers, L., Veltman, D. J., Van De Heyning, P., \& Sabbe, B. C. (2015). Tinnitus and anxiety disorders: A review. Hear Res. doi:10.1016/j.heares.2015.08.014

Peters, G.-J. Y. (2014). The alpha and omega of scale reliability and validity. The European Health Psychologist, 16(2), 56-69.

Pinto, P. C., Marcelos, C. M., Mezzasalma, M. A., Osterne, F. J., de Melo Tavares de Lima, M. A., \& Nardi, A. E. (2014). Tinnitus and its association with psychiatric disorders: systematic review. J Laryngol Otol, 128(8), 660-664. doi:10.1017/s0022215114001030

Preston, C. C., \& Colman, A. M. (2000). Optimal number of response categories in rating scales: reliability, validity, discriminating power, and respondent preferences. Acta Psychologica, 104(1), 1-15. doi:10.1016/S0001-6918(99)00050-5 
Roelofs, J., McCracken, L., Peters, M., Crombez, G., van Breukelen, G., \& Vlaeyen, J. S. (2004). Psychometric Evaluation of the Pain Anxiety Symptoms Scale (PASS) in Chronic Pain Patients. J Behav Med, 27(2), 167-183. doi:10.1023/ B:JOBM.0000019850.51400.a6

Roelofs, J., Sluiter, J. K., Frings-Dresen, M. H. W., Goossens, M., Thibault, P., Boersma, K., \& Vlaeyen, J. W. S. (2007). Fear of movement and (re)injury in chronic musculoskeletal pain: Evidence for an invariant two-factor model of the Tampa Scale for Kinesiophobia across pain diagnoses and Dutch, Swedish, and Canadian samples. Pain, 131(1-2), 181-190. doi:10.1016/j.pain.2007.01.008

Rubin, D. B. (1984). Bayesianly Justifiable and Relevant Frequency Calculations for the Applied Statistician. Ann. Statist., 12(4), 1151-1172. doi:10.1214/aos/1176346785

Schmidt, C. J., Kerns, R. D., Griest, S., Theodoroff, S. M., Pietrzak, R. H., \& Henry, J. A. (2014). Toward development of a tinnitus magnitude index. Ear Hear, 35(4), 476-484. doi:10.1097/aud.0000000000000017

Speckens, A. E. M., Spinhoven, P., Sloekers, P. P. A., Bolk, J. H., \& van Hemert, A. M. (1996). A validation study of the Whitely Index, the Illness Attitude Scales, and the Somatosensory Amplification Scale in general medical and general practice patients. J Psychosom Res, 40(1), 95-104. doi:10.1016/0022-3999(95)00561-7

Stewart, S. H., \& Watt, M. C. (2000). Illness Attitudes Scale dimensions and their associations with anxiety-related constructs in a nonclinical sample. Behav Res Ther, 38(1), 83-99. doi:10.1016/S0005-7967(98)00207-1

Sullivan, M. D., Katon, W., Dobie, R., Sakai, C., Russo, J., \& Harrop-Griffiths, J. (1988). Disabling tinnitus. Association with affective disorder. Gen Hosp Psychiatry, 10(4), 285-291.

Sullivan, M. J. L., Bishop, S. R., \& Pivik, J. (1995). The Pain Catastrophizing Scale: Development and validation. Psychological Assessment, 7(4), 524-532. doi:10.1037/1040-3590.7.4.524

Tyler, R. S., \& Baker, L. J. (1983). Difficulties experienced by tinnitus sufferers. J Speech Hear Disord, 48(2), 150-154. doi:10.1044/jshd.4802.150

van de Looij-Jansen, P. M., \& de Wilde, E. J. (2008). Comparison of Web-Based versus Paper-and-Pencil Self-Administered Questionnaire: Effects on Health Indicators in Dutch Adolescents. Health Services Research, 43(5 Pt 1), 1708-1721. doi:10.1111/ j.1475-6773.2008.00860.x

Vlaeyen, J. W. S., Crombez, G., \& Linton, S. J. (2016). The fear-avoidance model of pain. Pain, 157(8), 1588-1589. doi:10.1097/j.pain.0000000000000574

Vlaeyen, J. W. S., \& Linton, S. J. (2000). Fear-avoidance and its consequences in chronic musculoskeletal pain: a state of the art. Pain, 85(3), 317-332. doi:10.1016/S03043959(99)00242-0

Vlaeyen, J. W. S., \& Linton, S. J. (2012). Fear-avoidance model of chronic musculoskeletal pain: 12 years on. Pain, 153(6), 1144-1147. doi:10.1016/j.pain.2011.12.009 
Walt, N., Atwood, K., \& Mann, A. (2008). Does Survey Medium Affect Responses? An Exploration of Electronic and Paper Surveying in British Colombia Schools. Journal of Technology, Learning, and Assessment, 6(7). Retrieved from http://www.jtla.org website:

Wang, C.-C., Liu, K.-S., Cheng, C.-L., \& Cheng, Y.-Y. (2013). Comparison of web-based versus paper-and-pencil administration of a humor survey. Computers in Human Behavior, 29(3), 1007-1011. doi:https://doi.org/10.1016/j.chb.2012.12.029

Ware Jr, J. E., Kosinski, M., Gandek, B., Aaronson, N. K., Apolone, G., Bech, P., . . . Sullivan, M. (1998). The Factor Structure of the SF-36 Health Survey in 10 Countries: Results from the IQOLA Project. J Clin Epidemiol, 51(11), 1159-1165. doi:http://dx.doi. org/10.1016/S0895-4356(98)00107-3

Woods, C. (2002). Factor Analysis of Scales Composed of Binary Items: Illustration with the Maudsley Obsessional Compulsive Inventory. Journal of Psychopathology and Behavioral Assessment, 24(4), 215-223. doi:10.1023/A:1020770831134

Zeman, F., Koller, M., Schecklmann, M., Langguth, B., \& Landgrebe, M. (2012). Tinnitus assessment by means of standardized self-report questionnaires: Psychometric properties of the Tinnitus Questionnaire (TQ), the Tinnitus Handicap Inventory (THI), and their short versions in an international and multi-lingual sample. Health and Quality of Life Outcomes, 10, 128-128. doi:10.1186/1477-7525-10-128

Zöger, S., Svedlund, J., \& Holgers, K. M. (2006). Relationship between tinnitus severity and psychiatric disorders. Psychosomatics, 47(4), 282-288. doi:10.1176/appi.psy.47.4.282

Zwick, W. R., \& Velicer, W. F. (1986). Comparison of Five Rules for Determining the Number of Components to Retain. Psychol Bull, 99(3), 452-442. doi:10.1207/s15327906mbr1702_5

Author contributions: T.E.F. analysed data and wrote the paper; R.F.F.C. designed the study, collected the data and provided critical revision; E.V.d.B provided statistical advice and critical revision; J.W.S.V. provided critical revisions and study oversight. 


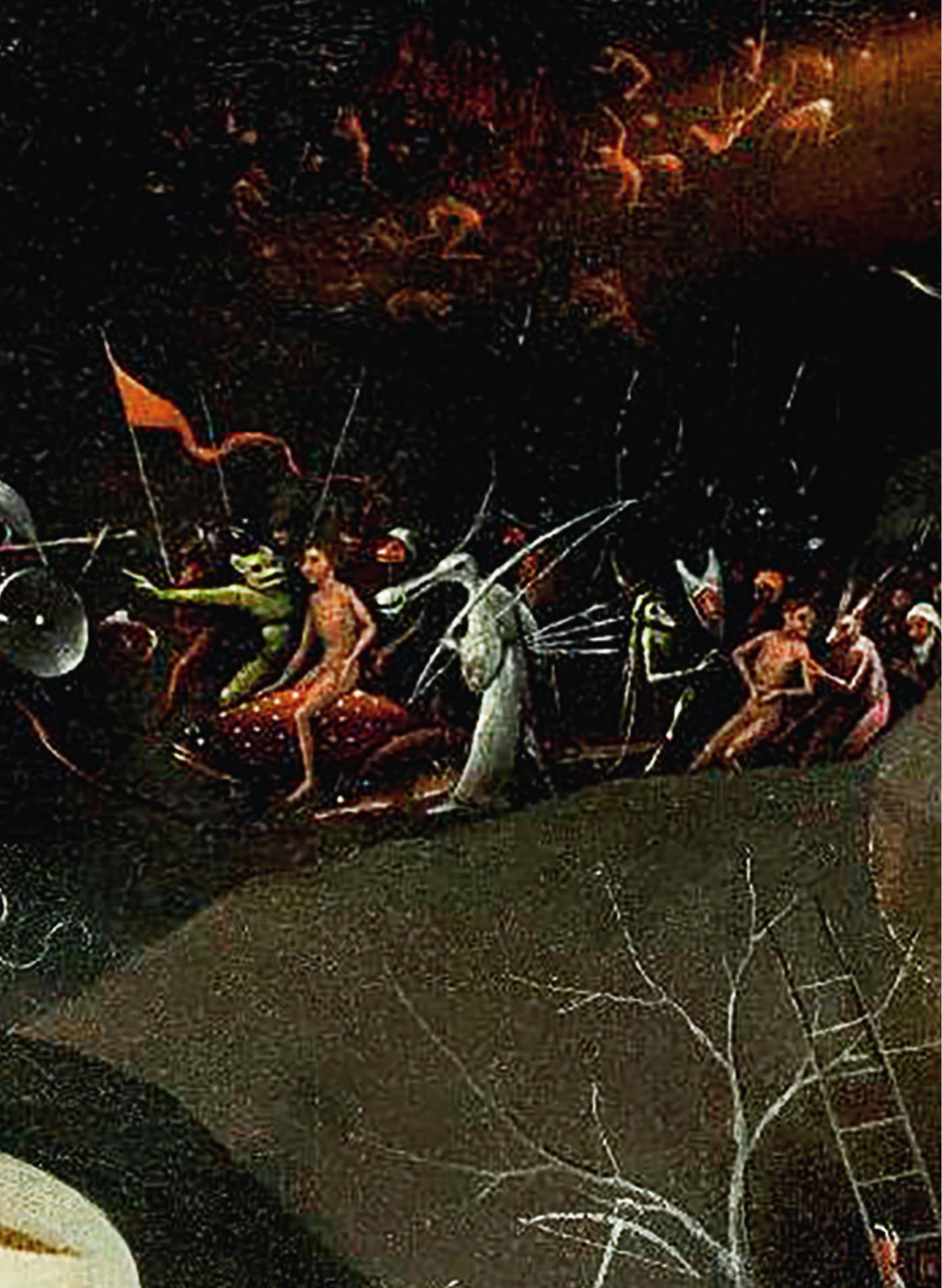




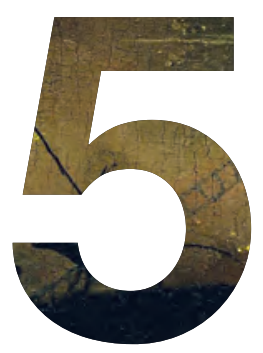

\section{PRAGMATIC UNCONTROLLED}

\section{STUDY OF SPECIALIZED COGNITIVE}

\section{BEHAVIOURAL THERAPY FOR}

\section{ADULTS WITH CHRONIC TINNITUS}

Fuller, T.E., van Breukelen, G.J.P., Vlaeyen, J.W.S., \& Cima, R.F.F. Pragmatic uncontrolled study of specialized cognitive behavioural therapy for adults with chronic tinnitus. Journal of Consulting and Clinical Psychology (under review). 


\section{Abstract}

Objective. Tinnitus is the perception of sound without an external source, affecting quality of life (QoL) that can cause severe distress in approximately $3 \%$ of the population. Randomized controlled trials (RCTs) of cognitive behavioral therapy (CBT) for tinnitus have demonstrated its effectiveness in improving QoL, but the effects of their implementation on a large scale in routine practice remains unknown. Therefore, the main purpose of this study was to examine the effects of a specialised CBT for tinnitus delivered in routine practice. Secondly, we wished to examine predictors of favourable outcome.

Methods. Four-hundred and three participants with chronic tinnitus referred to a tertiary audiological centre were included. The primary outcome was health-related quality of life as measured by the Health Utilities Index III (HUI-III) at 12-months. Secondary outcomes were self-reported levels of tinnitus-related distress, disability, affective distress and tinnitus-related catastrophizing and fear. Measures were completed preintervention, at 3-months, 8-months, and 12-months. Multilevel modelling (MLM) was used to examine effects and their predictors.

Results. MLM analyses revealed, with one exception, no relation between any baseline variable and outcome change over time. Most participants' improvement exceeded minimally clinical important difference (MCID) criteria for QoL, tinnitus-related handicap, and tinnitus distress.

Conclusions. This is the largest pragmatic study of specialized CBT for individuals with chronic tinnitus in routine care. The data complements results from RCTs and supports its implementation under "real world" conditions.

Keywords: Cognitive behavioral therapy, chronic tinnitus, effectiveness, implementation, Quality of Life

\section{Public health significance}

Testing cognitive behavioral therapy for tinnitus under randomized controlled conditions has previously established its efficacy in alleviating distress and interference in daily life. However, results from randomized controlled trials do not indicate to psychologists, policy makers, insurance companies or patients whether it would work in the "real world". This study demonstrates that cognitive behavioral therapy, tailored to patients suffering from chronic tinnitus, and delivered according to a stepped-care model, can be implemented successfully in routine care. This study was designed and conducted to resemble as closely as possible everyday practice (i.e. maximize external validity), but suffers from the absence of a wait-list control condition. 


\section{Introduction}

Developing and testing the effects of psychological interventions striving to be included in routine clinical practice and healthcare policy is a complex and challenging endeavour. Interventions typically need to demonstrate that they can work under ideal conditions (i.e. through efficacy or explanatory trials), are cost-effective and capable of being implemented under "real-world" conditions (i.e. through effectiveness or pragmatic trials) before they can be considered by policy makers, regulatory bodies and insurance companies (Thorpe et al., 2009) After all, implementing efficacious interventions poorly or recommending interventions without a credible evidence base risks scarce financial resources and harm to patients (Grimshaw, Eccles, Lavis, Hill, \& Squires, 2012). While some policymakers consider efficacy and pragmatic studies to be independent, they are better conceived as being on a continuum on which trade-offs between internal and external validity are made depending on the purpose of the trial (Gartlehner, Hansen, Nissman, Lohr, \& Carey, 2006; Sox \& Lewis, 2016).

The challenges of developing and subsequently implementing a new intervention for tinnitus are significant since assessment and intervention pathways are usually fragmented (Hoare \& Hall, 2011) and clinical guidelines are rare (Fuller et al., 2017). Tinnitus itself is a very common audiological symptom frequently correlated with older age and hearing loss, although only between 1-3\% of the population suffers from it (that is, so called bothersome tinnitus) (Davis \& El Refaie, 2000; Kim et al., 2015). Although there are a large variety of intervention options; psychological, sound, electrical and electromagnetic stimulation, tinnitus has no reliable cure (Folmer, Theodoroff, Martin, \& Shi, 2014). Accumulating evidence however suggests that CBT is an efficacious intervention in alleviating tinnitus related distress and interference (Fuller et al., 2020).

Although a recent systematic review and meta-analysis by Fuller et al. (2020) included 28 RCT's of CBT for tinnitus, there are relative few reports of effectiveness studies of CBT for tinnitus. Those that do exist have examined Internet-based CBT for tinnitus (Andersson \& Hedman, 2013; Kaldo-Sandstrom, Larsen, \& Andersson, 2004; Kaldo et al., 2013), audiologist delivered CBT (Aazh \& Moore, 2018), mindfulness-based CBT (McKenna, Marks, \& Vogt, 2018), and CBT for tinnitus related insomnia (Marks, McKenna, \& Vogt, 2019). To date, no study has yet examined the implementation of specialised CBT for tinnitus delivered in a stepped-care model of intervention (Cima et al., 2012). This is noteworthy since this two-step intervention (described below), when compared with 'treatment as usual', delivered under RCT conditions achieved small to medium effects sizes on the outcome measures (Cima et al., 2012). The results challenged a widespread belief amongst clinicians (e.g. audiologists, doctors and psychologists) that nothing could be done for tinnitus sufferers (Langguth, 2012).

The aim of this study was to investigate whether changes in quality of life (QoL) and tinnitus related outcome measures occurred over a 12-month period in participants who completed a specialised CBT for tinnitus intervention in routine tinnitus care and under "real world" conditions. Given the aim of the study, a randomized controlled 
trial design was not appropriate and we used an observational study design with data collected at four time points. It was hypothesized that adult participants with tinnitus receiving stepped care specialised CBT for tinnitus have improved QoL, and decreased levels of tinnitus related distress, catastrophizing and fear after completing specialised CBT for tinnitus.

\section{Method}

\section{Study design and ethics}

This observational study included 403 participants between January 2014 and November 2016. Outcome data was collected at baseline (T0), at the end of step 1 (i.e. 3-months after baseline; T1); at 8-months after baseline (i.e. for some, after completion of step 2; T2); and, at 12-months after baseline (T3). Figure 1 illustrates the flow of participants throughout the study. The study was conducted in accordance with the principles of the Declaration of Helsinki, approved by the Ethical Review Committee Faculty of Psychology and Neuroscience, Maastricht University (approval number: ECP152 05_12_2014) and registered at clinicaltrials.gov (NCT04310605). Participants did not report any harms or adverse events during the course of the study.

\section{Participants}

Participants were recruited from five audiological centres located in the province of Brabant, The Netherlands. People were eligible to participate if they were: 17 years or older, primarily seeking help for tinnitus related distress or its interference in daily activities, and who were able to communicate in Dutch. All prospective participants received detailed written information about the study and the associated requirements of participating. Participation in the study did not require any time commitment beyond that of the intervention. (Note: completing questionnaires was considered part of the intervention protocol, a requirement of funding from health insurers, and used as a quality control mechanism in the course of the study.)

\section{Measures}

Primary outcome measure

The Health Utilities Index-III (HUI; Feeny et al., 2002; Horsman, Furlong, Feeny, \& Torrance, 2003) is a 17-item measure designed to assess health related QoL. It has eight subscales/dimensions (vision, hearing, speech, ambulation, dexterity, emotion, cognition and pain complaints) that have five or six rating options ranked, for example, from "highly impaired" to "normal". Utility scores on the HUI-III range from -.36 to 1.00. The HUI-III has a strong theoretical basis, is widely used in clinical research, and considered a reliable and valid measure (Horsman et al., 2003). The HUI-III has been shown to have adequate responsiveness when used with tinnitus patients (Maes, Joore, Cima, Vlaeyen, \& Anteunis, 2011). The internal reliability of the HUI-III in this was study was excellent $(a=0.88,95 \% \mathrm{Cl}[0.87-0.90])$.

\section{Secondary outcome measures}

Tinnitus Handicap Inventory (THI; Newman, Jacobson, \& Spitzer, 1996; Newman, Sandridge, \& Jacobson, 1998) The THI is a 25-item measure of the impact of tinnitus on daily 
life that includes three subscales (mental, social/occupational and physical functioning; emotional impact; catastrophic responses to tinnitus). The total score of the THI ranges from 0-100 with higher scores indicating greater levels of impact on daily life. The internal reliability of the $\mathrm{THI}$ in this was study was excellent $(\mathrm{a}=0.96,95 \% \mathrm{Cl}[0.95-0.96])$.

Hospital Anxiety and Depression Scale (HADS; Zigmond \& Snaith, 1983) The HADS is a widely used measure of psychological distress in people experiencing a concurrent physical health condition. It has 14 items and respondents use a Likert-type scale to indicate how often they have had a particular feeling in the previous week (e.g. "I feel tense or wound up"). Each item is scored from 0-3 with lower scores indicating better psychological functioning. A large number of studies have investigated the factor structure of the HADS with some confirming a two- factor structure while others have found one, three or four factors (Bjelland, Dahl, Haug, \& Neckelmann, 2002) leading to some debate about its utility (Coyne \& van Sonderen, 2012a, 2012b). For this study the HADS total score (range 0-42) was used as a unidimensional measure of emotional distress. The internal reliability of the HADS in this was study was excellent $(a=0.94$, $95 \% \mathrm{Cl}[0.93-0.94])$.

Tinnitus Catastrophizing Scale (TCS; Cima, Crombez, \& Vlaeyen, 2011) The TCS was used to assess the degree to which people thought or expected the worst about tinnitus. The TCS is a 13-item measure based on the Pain Catastrophizing Scale (Sullivan, Bishop, \& Pivik, 1995) and respondents use a five- point scale to indicate the degree to which statements applies to them (e.g. It's terrible and I think it's never going to get any better). The total score on the TCS ranges from 0-65. The internal reliability of the TCS in this study was excellent $(a=0.95,95 \% \mathrm{Cl}[0.94-0.95])$.

Fear of Tinnitus Questionnaire. The FTQ is a 17-item self-report measure intended and designed to assess respondents' level of fear regarding their tinnitus. Items in the questionnaire are presented as a series of statements (e.g. "I am afraid that my tinnitus will become worse") from which respondents are asked to indicate if it is applicable to their current situation. Each statement receives a score of 1 when applicable (total score range $0-17$ ). The FTQ has been shown to be sensitive to change following intervention (Cima et al., 2012) and have good psychometric properties (Fuller, Cima, Van den Bussche, \& Vlaeyen, 2019). The internal reliability of the FTQ in this was study was excellent $(\alpha=0.91,95 \% \mathrm{Cl}[0.90-0.92])$.

The Tinnitus Questionnaire (TQ; Hallam, Jakes, \& Hinchcliffe, 1988) is a self-report questionnaire designed to asses distress and interference in daily activities associated with tinnitus. It has 52 items and uses a three-point scale to indicate levels of distress on six subscales. The total possible score on the TQ ranges from 0-84. The TQ has high internal consistency, convergent and discriminant validity and is sensitive to change (Baguley, Humphriss, \& Hodgson, 2000; Zeman, Koller, Schecklmann, Langguth, \& Landgrebe, 2012). The internal reliability of the TQ in this was study was excellent $(a=0.96,95 \% \mathrm{Cl}[0.95-0.96])$. 
The Tinnitus Disability Index (TDI; Cima, Vlaeyen, Maes, Joore, \& Anteunis, 2011) is a 7 item self-report questionnaire that assesses the level of interferences in daily activities attributed to tinnitus. Respondents use an 11-point scale to indicate the level of interference ranging from 0 no disability, to 10 total disability. The total score ranges from 0 to 70 with higher scores indicating higher levels of interference. The TDI has been shown to be reliable over time, and higher scores on the measure have been shown to be correlated with higher ratings of tinnitus intensity and distress, and lower levels of QoL. In the present study the internal reliability of the TDI was excellent $(a=0.90,95 \%$ $\mathrm{Cl}[0.89-9.92])$.

\section{Procedure}

Preparation/therapist training

Prior to implementing the intervention, psychologists, clinical physicists in audiology, audiometricians, movement therapists, physical therapists and social workers) at the audiology rehabilitation centre who expressed interest in participating in the study underwent training in specialised CBT for tinnitus. Two audiologists, four audiometric technicians, one social worker and three psychologists participated in a one week (36 hours) intensive step-1-treatment training course. This consisted of observing, practicing and providing the step-1 treatment elements per discipline, participation in multidisciplinary case-triage and instructions on the organizational and logistic aspects of the intervention. The step-2 trainees followed a 6-month training course at Adelante Audiology and Communication, Hoensbroek, The Netherlands. In sum, training was extensive and consisted of observation of therapeutic methods, practicing under supervision, and performing the intervention independently. Members of the trainee team were also provided with protocols describing in detail the aims, instructions and primary therapeutic processes for each session for the respective intervention pathway. [Details of the training are provided in Chapter 5 Supplementary Information File 1, p.3-4.]

Description of the intervention

Step 1 of the intervention comprised individual audiometric diagnostics and counselling about hearing and tinnitus with an audiologist (one hour); an educational group session with a maximum of 10 participants and their partners (two hours); and psychological assessment regarding tinnitus and its impact on daily life (one hour). Each patient was discussed in a multidisciplinary case-discussion (10 min) after step 1 was concluded

Step 2 included psycho-education; exposure therapy to reduce fear and avoidance of stimuli that predict the occurrence or the increase of tinnitus complaints, and extinction of associated safety-seeking behaviors; movement therapy and attentionfocused/mindfulness exercises to facilitate the exposure to tinnitus-related stimuli; cognitive restructuring of catastrophic thoughts about the meaning of tinnitus and the consequences of living with tinnitus; attention-redirecting techniques; stress reduction; and relaxation techniques (e.g. progressive muscle relaxation). Step 2 group sessions were two-hours long weekly, and held over a 12-week period. The eligibility for step 2 included a baseline TQ score of 47 or more in addition to a perceived need for further help at the end of step 1 by participants and the treatment team (Additional details of step 2 intervention are provided Chapter 5, Supplementary Information File 1, p. 4-5). 
Implementation

During the implementation phase, frequent supervisory visits and weekly onlinemeetings between expert and novice therapists were conducted to address clinical issues. Project team meetings were also held on a monthly basis to enable the project coordinators, management of the rehabilitation centre and the researchers to monitor the progress of the inclusion, intervention allocation, and intervention fidelity.

\section{Statistical analyses}

Predicting dropout by three months and predicting receipt of step 2

We conducted two logistic regression analyses prior to examining changes in outcome measures over time. The first was conducted to inform our understanding of participants' characteristics of those who did (or did not) complete outcome measures beyond baseline measurement; and the second was a manipulation check of the (main) criteria for participants to receive step 2 . The first regression included the total sample ( $N=403$ ) to examine what factors predicted dropout by Time 1; and, the second included the total sample, minus dropouts at T1. We expected that baseline TQ score would be a predictor of step 2 intervention if the study protocol/criteria were followed. However, we did not know if other variables measured at baseline would also predict the likelihood of undertaking step 2.

For the purposes of the first logistic regression (predictors of dropout) participants who did not complete any outcome measures after baseline were considered to have "dropped out" (see Figure 1). The regression model included as predictors of dropout: demographic and clinical variables (i.e. age, tinnitus duration, hearing loss, education, gender, employment status, season in which participants commenced intervention), and baseline scores on outcome measures (i.e. HUI-III, THI, HADS, TCS, FTQ, TQ, TDI). In the second logistic regression (predictors of step 2 involvement), the scores on the outcome measures at three months after baseline (i.e. T1) were also included as predictors in addition to those used in the first logistic regression examining dropout. Participants who had dropped out were not included in this analysis.

For both logistic regressions, a decision rule for reducing/simplifying the model was applied whereby predictors with $p$ values greater than .05 were removed in a stepwise fashion. Using an iterative process, and following the removal of the variable with the highest $p$ value, the model was re-run and $p$ values examined until the model only included variables with $p$ values less than $a=.05$ (two-tailed). To compensate for multiple testing, a Bonferroni correction was applied for drawing conclusions, that is, alpha was divided by the number of predictors in the initial full model (14), giving: $.05 / 14=.0036$. Since the Bonferroni correction is considered an overcorrection, we rounded it up slightly to set the alpha at .005 in order to minimize the chance of type 2 errors.

\section{Examining changes over time}

The results from the RCT of specialised CBT compared to usual care for tinnitus indicated that participants in the specialised care condition improved more than those receiving usual care, and that the improvement in each treatment group was maintained over the follow-up period (Cima et al., 2012). Additionally, tinnitus-related fear appeared 
to mediate part of the intervention effect on other outcomes (Cima, van Breukelen, \& Vlaeyen, 2017). While we might expect that participants will improve over the course of the intervention, without a control group we cannot specifically assess treatment effects (or mediators thereof) per se. Given that, no specific hypotheses regarding these effects were made.

As in the RCT, mixed (multilevel) regression was used to examine if changes in outcomes occurred over the intervention and follow-up period, and if so, which variables predicted the change. Mixed regression is able to account for nesting of data (i.e. repeated measures within participants), and is robust against missing outcome data. This means that all patients with at least one measurement can be included into the analysis and that the results are valid even if outcome missingness depends on any measurement prior to dropout (Verbeke \& Molenberghs, 2000). We conducted three sets of analyses on the primary (HUI-III) and each of the secondary outcomes (THI, HADS, TCS FTQ, $\mathrm{TQ}$ and TDI). The first set examined participants who only completed step 1 of CBT for tinnitus, i.e. baseline (T0) to 3-months (T1). This was done to assess whether there was any interaction between the predictor variables included in the model and time, or equivalently, to assess predictors of change from baseline to T1. The second set included the same participants but now the outcome measures at all time points. The third set of analyses focused on participants who undertook both step 1 and 2 at each time point.

Before conducting the mixed regressions, we checked for collinearity between predictor variables. All predictor variables had a Variance Inflation Factor (VIF) below 10 indicating absence of collinearity 10 (VIF's range: $1.04-6.44$ ), and hence were included into the regression analyses. For each set of analyses, we initially conducted maximum likelihood (ML) estimation, assuming an unstructured covariance matrix of the repeated measures (which is the most flexible and thus safe choice). A Bonferroni correction was applied to compensate for multiple testing (i.e. testing 14 predictor ${ }^{\star}$ time interactions on each of the seven outcomes), giving an alpha of .05/98, which was rounded upward to.001 as Bonferroni gives an overcorrection.

Model selection for step 1 only participants, and step 1 and 2 participants respectively, proceeded through a stepwise process. Models initially included the set of predictor variables, time (with baseline as reference category and a dummy indicator for each other time point) and the interaction of each predictor with time. We ran a model and subsequently deleted the predictor by time interaction with the highest $p$-value and checked its accuracy by a likelihood ratio test. This process continued until all predictor by time interactions either were below the .001 criterion or were deleted from the model. As an additional check for statistically significant interaction effects, we also re-introduced each predictor by time interaction separately into the final model to examine if any reached the level of significance (i.e. we started with a backward stepwise regression from the full model, followed by a forward regression from the final model). We used restricted maximum likelihood (REML) regression to produce a final estimate of effects of the predictor variables and their standard errors, as REML is the best method for estimating standard errors, but ML was needed for likelihood ratio comparisons between models (Verbeke \& Molenberghs, 2000). Histograms were used to check for normality of the residual distribution and outliers in the residuals per 
time point. If outliers were identified, the effect of removing the respective participants from the analysis was checked by re-running the particular model. In all analyses with outliers, removing participants from the analysis did not affect the predictor selection or effects. Therefore, we report the results using the data set including all participants.

Participants were post hoc classified as having improved or not using observed data points, and on available minimal clinically important difference (MCID) criteria for HUIIII, THI, TQ and HADS. Specifically, participants were classified as 'improved' if there was at least: an increase of .03 for HUI-III (Horsman et al., 2003; Marra et al., 2005); a decrease of 7 points on the THI (Zeman et al., 2012); and a decrease of 12 points on the TQ (Hall, Mehta, \& Argstatter, 2018). In the absence of tinnitus-specific data, participants were also classified as 'improved' on HADS if their total score decreased by 1.7 points (reference group cardiovascular patients; Lemay, Tulloch, Pipe, \& Reed, 2019) and 1.5 points (reference group chronic obstructive pulmonary disease patients; Puhan, Frey, Büchi, \& Schünemann, 2008). For each outcome, the percentage of participants who improved was calculated between baseline and 3-months, baseline and 8-months and baseline and 12-months.

\section{Results}

\section{Participant flow and sample characteristics}

All participants completed step 1 of the intervention, with $42.4 \%$ subsequently entering into step 2, and $40.4 \%$ not requiring further intervention after step1 (see Figure 1). Although the main criterion for entering step 2 was a baseline TQ score greater than or equal to 47, actually 25 of the 171 participants in step 2 (14.6\%) had a baseline TQ score below this level. Participants who had suffered from tinnitus for an average of 54 months, were more likely to be male, employed, perceive tinnitus in both ears, and did not use a hearing aid. Nearly $30 \%$ (28.9\%) of the participants who were employed received sickness benefits (Tables 1 and 2). Sixty-nine of 403 participants (17.1\%) did not complete any outcome measures at 3-, 8-, or 12-months after baseline. Although they did not complete outcome measures, most completed some or all treatment elements of step 1. Specifically, $68(98.5 \%)$ participants attended an appointment with the audiologist, 67 (97.1\%) attended the tinnitus information session and $41(59.4 \%)$ participants attended the appointment with the psychologist. 
Chapter 5

Figure 1. Participant Flow Diagram

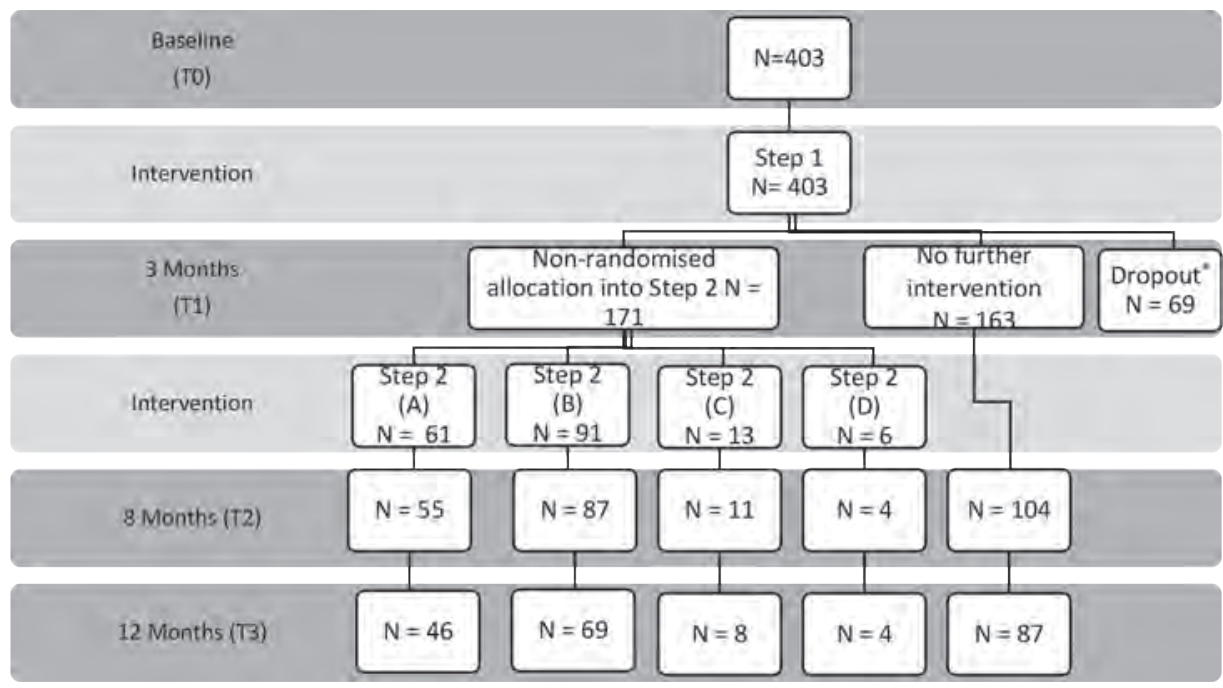

Note: *Participants who completed baseline measures but not T1, T2 or T3.

Table 1. Participant Characteristics (Continuous Variables)

\begin{tabular}{|c|c|c|c|c|c|}
\hline Characteristic & & $\mathrm{M}$ & SD & SE & $\mathrm{N}$ \\
\hline Age & & 54.5 & 12.1 & .60 & 403 \\
\hline Tinnitus duration & (months) & 54.2 & 89.2 & 4.5 & 397 \\
\hline \multirow[t]{2}{*}{ Hearing loss } & $\begin{array}{l}\text { Fletcher Index - Right - High } \\
-1,2,4 \mathrm{kHz}\end{array}$ & 29.4 & 21.6 & 1.1 & 400 \\
\hline & $\begin{array}{l}\text { Fletcher Index - Left - High - } \\
1,2,4 \mathrm{kHz}\end{array}$ & 30.0 & 22.2 & 1.1 & 399 \\
\hline
\end{tabular}

Table 2. Participant Characteristics (Categorical Variables)

\begin{tabular}{llll}
\hline Characteristic & N (\% of responses) & $\begin{array}{l}\text { Missing data } \\
\text { N (\% of total sample) }\end{array}$ \\
\hline Gender & & 0 \\
& Female & $157(39)$ & \\
Male & $246(61)$ & $227(56.3)$ \\
& & \\
& Secondary & $10(5.7)$ & \\
& Vocational training & $64(36.4)$ & \\
& University & $16(9.1)$ & \\
& Applied Sciences & $35(19.9)$ & \\
& Other & $51(28.9)$ & \\
&
\end{tabular}


Table 2. Continued.

\begin{tabular}{|c|c|c|c|}
\hline Characteristic & & $\mathrm{N}$ (\% of responses) & $\begin{array}{l}\text { Missing data } \\
\mathrm{N}(\% \text { of total sample) }\end{array}$ \\
\hline \multirow[t]{3}{*}{ Employed } & & & $12(3.0)$ \\
\hline & Yes & 238 (60.9) & \\
\hline & No & $153(39.1)$ & \\
\hline \multirow[t]{3}{*}{ Sickness benefits } & & & $164(40.7)$ \\
\hline & Yes & $69(28.9)$ & \\
\hline & No & $170(71.1)$ & \\
\hline \multirow[t]{7}{*}{ Location of tinnitus ${ }^{\star *}$} & & & $18(4.5)$ \\
\hline & Left side & $107(21.0)$ & \\
\hline & Right side & $68(13.1)$ & \\
\hline & Both ears & 199 (38.9) & \\
\hline & Middle of head & $33(6)$. & \\
\hline & Whole head & $84(16.4)$ & \\
\hline & Other & $21(4.1)$ & \\
\hline \multirow[t]{3}{*}{ Use of hearing aid } & & & $8(2.0)$ \\
\hline & Yes & $73(18.4)$ & \\
\hline & No & $322(81.5)$ & \\
\hline \multirow{3}{*}{$\begin{array}{l}\text { Use of other hearing } \\
\text { related aid }\end{array}$} & & & $4(1.0)$ \\
\hline & Yes & $27(6.8)$ & \\
\hline & No & $372(93.2)$ & \\
\hline
\end{tabular}

Note. *Education was recoded into: 1 = 'low'; 2 = middle-high'; and, 3 = 'other \& missing' for use in the logistic and mixed regression analyses respectively.

${ }^{* *}$ multiple responses were possible to this question hence $\mathrm{N}>403$.

\section{Predicting dropout at three months}

The final logistic regression model for dropout at 3-months explained $14.3 \%$ of the dropout (Nagelkerke $R^{2}$ ), and classified $84.4 \%$ of the cases correctly in terms of being a dropout or not (Note, however that a model with no predictors at all classified $83 \%$ of dropouts correctly by using the data that $17 \%$ dropped out). When using alpha $=.005$ for drawing conclusions in view of multiple testing, the final model revealed that a younger age and lower THI baseline score were associated with an increased likelihood of dropping out (Table 3). 
Chapter 5

Table 3. Final/Reduced Logistic Regression Predicting Dropout by 3-Months after Baseline. (N=403)

\begin{tabular}{|c|c|c|c|c|c|c|c|}
\hline & & B & S.E. & Wald & Df & Sig. & $\operatorname{Exp}(B)$ \\
\hline \multirow[t]{12}{*}{ Step $1^{a}$} & Age & -.050 & .012 & 16.384 & 1 & .000 & .952 \\
\hline & HUI baseline score & -1.474 & .564 & 6.828 & 1 & .009 & .229 \\
\hline & THI baseline score & -.035 & .012 & 8.502 & 1 & .004 & .966 \\
\hline & TCS baseline score & .043 & .021 & 4.241 & 1 & .039 & 1.044 \\
\hline & Education* & & & 8.312 & 2 & .016 & \\
\hline & Education(1) & -.110 & .494 & .050 & 1 & .823 & .895 \\
\hline & Education(2) & .844 & .415 & 4.137 & 1 & .042 & 2.325 \\
\hline & $\begin{array}{l}\text { Season in which Step } 1 \\
\text { started }^{\star \star}\end{array}$ & & & 11.188 & 3 & .011 & \\
\hline & $\begin{array}{l}\text { Season in which Step } 1 \\
\text { started(1) }\end{array}$ & -1.069 & .524 & 4.166 & 1 & .041 & .343 \\
\hline & $\begin{array}{l}\text { Season in which Step } 1 \\
\text { started(2) }\end{array}$ & .468 & .383 & 1.490 & 1 & .222 & 1.597 \\
\hline & $\begin{array}{l}\text { Season in which Step } 1 \\
\text { started(3) }\end{array}$ & -.054 & .481 & .013 & 1 & .910 & .947 \\
\hline & Constant & -2.256 & .506 & 19.871 & 1 & .000 & .105 \\
\hline
\end{tabular}

Note: a. Variable(s) entered on step 1: Age, HUI baseline score, THI baseline score, TCS baseline score, Education, Season in which Step 1 started.

b. *Education was recoded into: 1 = 'low'; 2 = middle-high'; and, 3 = 'other \& missing'; reference category $=3 .{ }^{*}$ Season in which step 1 started was coded as: $1=$ Spring; $2=$ Summer; $3=$ Autumn; $4=$ Winter (reference category).

\section{Predicting who would receive step 2 of specialised CBT for tinnitus}

We used logistic regression to examine the variables predicting participants who received step 2 . The chi-square test of the final model was statistically significant $\mathrm{X}^{2}(4)=95.722, \mathrm{p}<.001$ and explained $33.2 \%$ (Nagelkerke $R^{2}$ ) of the variance in the prediction of entering step 2 of the CBT intervention. The final model correctly predicted $72.5 \%$ of cases (to compare, the model without predictors correctly predicted $51.2 \%$ of cases). Higher scores on QoL (HUI-III) and tinnitus handicap (THI) at baseline, and on tinnitus distress (TQ) scores at T1 were associated with increased likelihood of participating in step 2 of the intervention (Table 4). 
Table 4. Final/Reduced Model of Logistic Regression Predicting Participation in Step 2 of Specialised CBT for Tinnitus $(\mathrm{N}=334)$

\begin{tabular}{llllllll}
\hline & & B & S.E. & Wald & Df & Sig. & Exp(B) \\
\hline Step 1 $1^{\mathrm{a}}$ & HUl baseline score & 2.258 & .538 & 17.638 & 1 & .000 & 9.562 \\
& THI baseline score & .034 & .011 & 9.533 & 1 & .002 & 1.034 \\
& FTQ baseline score & -.105 & .052 & 4.021 & 1 & .045 & .901 \\
& TQ score at T1 & .056 & .011 & 26.612 & 1 & .000 & 1.057 \\
& Constant & -2.685 & .540 & 24.718 & 1 & .000 & .068 \\
\hline
\end{tabular}

Note: a. Variable(s) entered on step 1: HUI baseline score, THI baseline score, FTQ baseline score, TQ score at T1 (3 months after baseline)

\section{Changes over time on outcome measures}

The primary aim of this research was to investigate participants' change over time and predictors of change. Observed mean and standard deviations on the respective outcome measures of interest by time and intervention trajectory are shown in Table 5. In sum, the observed scores across all outcomes reveal that, at a group level, participants improve over time. Note however, that the time courses might be biased due to the dropout (17.1\% after baseline measures) as each mean (and SD) is based on the observed cases at that time point. Therefore, plots of predicted values based on mixed regression including dropouts were generated to correct for this bias as much as possible.

Table 5. Mean Observed Score and Standard Deviations on Outcome Measures by Time Point and Intervention.

\begin{tabular}{|c|c|c|c|c|c|c|c|c|c|c|c|c|c|}
\hline \multirow{2}{*}{$\begin{array}{l}\text { Treatment } \\
\text { group }\end{array}$} & \multirow{2}{*}{ Outcome } & \multicolumn{3}{|c|}{ Baseline (T0) } & \multicolumn{3}{|c|}{3 months (T1) } & \multicolumn{3}{|c|}{8 months (T2) } & \multicolumn{3}{|c|}{12 months (T3) } \\
\hline & & Mean & SD & $\mathrm{N}$ & Mean & SD & $\mathrm{N}$ & Mean & SD & $\mathrm{N}$ & Mean & SD & $\mathrm{N}$ \\
\hline \multirow{7}{*}{$\begin{array}{l}\text { ते } \\
\text { o } \\
\frac{0}{\circ} \\
\frac{0}{d} \\
\dot{\omega}\end{array}$} & HUI-III & 0.53 & 0.31 & 231 & 0.62 & 0.29 & 162 & 0.65 & 0.28 & 104 & 0.64 & 0.29 & 87 \\
\hline & THI & 42.95 & 22.49 & 231 & 32.06 & 22.79 & 163 & 27.38 & 22.41 & 104 & 25.24 & 22.05 & 87 \\
\hline & HADS & 6.82 & 4.05 & 231 & 5.65 & 3.92 & 163 & 5.1 & 3.88 & 104 & 4.68 & 3.8 & 87 \\
\hline & TCS & 21.62 & 11.78 & 231 & 15.77 & 11.91 & 162 & 13.03 & 11.39 & 104 & 11.15 & 10.16 & 87 \\
\hline & FTQ & 7.69 & 3.83 & 231 & 5.37 & 3.82 & 163 & 4.3 & 3.59 & 104 & 3.89 & 3.62 & 87 \\
\hline & TQ & 51.2 & 19.11 & 230 & 39.51 & 20.3 & 163 & 34.71 & 19.95 & 104 & 32.16 & 18.13 & 87 \\
\hline & TDI & 21.9 & 16.0 & 231 & 16.3 & 15.4 & 161 & 14.5 & 16.8 & 104 & 14.3 & 15.9 & 87 \\
\hline \multirow{7}{*}{$\begin{array}{l}\sim \\
\infty \\
\frac{0}{0} \\
\frac{0}{\omega}\end{array}$} & HUI-III & 0.53 & 0.3 & 172 & 0.54 & 0.28 & 169 & 0.57 & 0.3 & 157 & 0.62 & 0.25 & 126 \\
\hline & THI & 57.33 & 18.33 & 172 & 52.96 & 19.67 & 169 & 45.26 & 20.62 & 157 & 39.86 & 20.3 & 127 \\
\hline & HADS & 8.9 & 3.7 & 172 & 8.28 & 3.78 & 170 & 7.57 & 3.77 & 157 & 6.71 & 3.61 & 127 \\
\hline & TCS & 26.92 & 9.61 & 172 & 24.08 & 10.55 & 169 & 19.18 & 10.96 & 155 & 16.97 & 11.3 & 127 \\
\hline & FTQ & 9.11 & 3.37 & 172 & 8.04 & 3.62 & 170 & 6.43 & 3.8 & 157 & 5.42 & 3.78 & 127 \\
\hline & $\mathrm{TQ}$ & 62.47 & 14.98 & 172 & 57.11 & 15.62 & 171 & 47.94 & 18.7 & 154 & 43.44 & 18.63 & 126 \\
\hline & TDI & 29.6 & 15.3 & 172 & 26.1 & 14.4 & 168 & 24.0 & 16.3 & 153 & 20.0 & 14.3 & 125 \\
\hline
\end{tabular}


Chapter 5

Table 5. Continued.

\begin{tabular}{|c|c|c|c|c|c|c|c|c|c|c|c|c|c|}
\hline \multirow{2}{*}{$\begin{array}{l}\text { Treatment } \\
\text { group }\end{array}$} & \multirow[b]{2}{*}{ Outcome } & \multicolumn{3}{|c|}{ Baseline (T0) } & \multicolumn{3}{|c|}{3 months (T1) } & \multicolumn{3}{|c|}{8 months (T2) } & \multicolumn{3}{|c|}{12 months (T3) } \\
\hline & & Mean & SD & $\mathrm{N}$ & Mean & SD & $\mathrm{N}$ & Mean & SD & $\mathrm{N}$ & Mean & SD & $\mathrm{N}$ \\
\hline \multirow{7}{*}{ 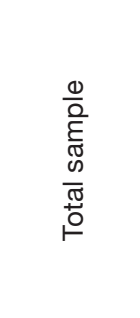 } & HUI-III & 0.53 & 0.3 & 403 & 0.58 & 0.29 & 331 & 0.6 & 0.3 & 261 & 0.63 & 0.27 & 213 \\
\hline & THI & 49.09 & 21.98 & 403 & 42.7 & 23.67 & 332 & 38.14 & 23.04 & 261 & 33.92 & 22.18 & 214 \\
\hline & HADS & 7.71 & 4.03 & 403 & 6.99 & 4.06 & 333 & 6.59 & 4 & 261 & 5.89 & 3.81 & 214 \\
\hline & TCS & 23.88 & 11.21 & 403 & 20.01 & 11.97 & 331 & 16.71 & 11.51 & 259 & 14.6 & 11.2 & 214 \\
\hline & FTQ & 8.3 & 3.7 & 403 & 6.73 & 3.94 & 333 & 5.58 & 3.86 & 261 & 4.79 & 3.78 & 214 \\
\hline & $\mathrm{TQ}$ & 56.02 & 18.31 & 402 & 48.52 & 20.07 & 334 & 42.61 & 20.25 & 258 & 38.84 & 19.21 & 213 \\
\hline & TDI & 25.2 & 16.2 & 403 & 21.3 & 15.7 & 329 & 20.1 & 17.1 & 257 & 17.7 & 15.2 & 212 \\
\hline
\end{tabular}

Note: $\mathrm{T} 0=$ baseline; $\mathrm{T} 1=3$ months after baseline; $\mathrm{T} 2=8$ months after baseline; $\mathrm{T} 3=12$ months after baseline

Mixed (multilevel) regression analyses were used to examine what variables might predict change in the outcome over the 12-month follow-up. These analyses generate a large amount of output. For the sake of transparency and completeness, this is presented in Chapter 5 Supplementary Information Files 1 (SIF 5.1). Tables 1 to 3 in SIF 5.1 (p. 6-11) present a summary of the statistically significant predictors of change (predictor by time effect) and of average outcome (predictor main effect) per participant subgroup (step 1only / step 1\&2), per outcome. Briefly, no significant predictor by time interactions were found, except for an interaction of baseline TCS score with time with respect to the outcome HADS in the group of participants who completed both step 1\&2. This suggests that the effect of time on HADS score is dependent on the baseline TCS score. This means that the HADS change over time is related to the baseline TCS score. The results also reveal that no single predictor was consistently associated with all outcomes in both participant groups. The absence of a consistent pattern also applied to near-significant predictors. Log ratio tests of initial full model (all interactions in) versus final model (no interactions), using ML estimation, were also conducted for each outcome as another check for interactions. These tests confirmed the absence of predictor by time interactions except for a possible season by time effect on TQ for participants who undertook step 1 only (SIF 5.2).

Figure 2 shows the predicted values based on the final REML models for the primary outcome (HUI-III) for the two groups of participants. On the average, step 1 participants improved between 0 and 3 months and remained stable thereafter, whereas step1\&2 participants showed improvement between 3 and 12 months, that is, after step 2 onset. The scenario is somewhat different for the secondary outcomes (Figures 1 to 6, SIF 5.1, p. 12-17), where for each outcome, participants who were allocated into the step1 only, intervention started better in QoL and finished better than participants who received step 1\&2. This difference between groups can be understood since stepped care was organized to allocate step 2 to those who needed it most (i.e. higher TQ score at baseline). 
Classifying participants as 'improved' (or not) using MCID criteria, from baseline to 12-months and observed data, revealed that over half of participants improved on QoL (i.e. HUI-III, $n=115 / 213,54.0 \%$ ), tinnitus impact on daily life (i.e. THI, $n=145 / 214$, $67.8 \%$ ), and tinnitus distress (i.e. TQ, $n=133 / 213,62.45 \%$ ). Just under and slightly over half respectively also 'improved' on levels of psychological distress when using cardiovascular patients' (i.e. HADS, n=100/214, 46.7\%) and when using COPD patients' (i.e. HADS, $n=115 / 214,53.7 \%$ ) MCID criteria. On these four outcomes, the percentage of participants who improved increased over time (Table 6).

Figure 2. Predicted HUI-III Scores by Time Using Final (REML) Model

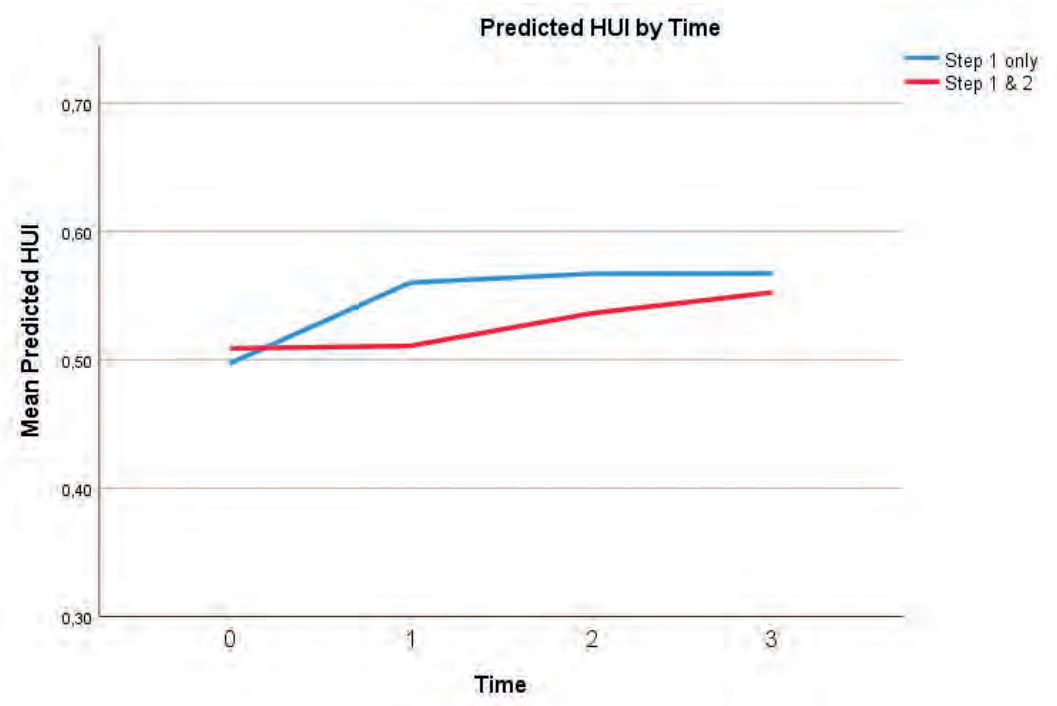


Chapter 5

Table 6. Numbers of Participants Improved by Outcome Measure

\begin{tabular}{lllll}
\hline Outcome & MCID & $\begin{array}{l}\text { Baseline to } 3 \text { months } \\
\%(n \text { improved/n at T1) }\end{array}$ & $\begin{array}{l}\text { Baseline to } 8 \text { months } \\
\text { (n improved/n at T2) }\end{array}$ & $\begin{array}{l}\text { Baseline to 12-months } \\
\text { ( } \mathrm{n} \text { improved/n at T3) }\end{array}$ \\
\hline HUI & .03 & $46.8(155 / 331)$ & $51.0(133 / 261)$ & $54.0(115 / 213)$ \\
THI & 7 & $43.7(145 / 332)$ & $59.0(154 / 261)$ & $67.8(145 / 214)$ \\
TQ & 12 & $32.9(110 / 334)$ & $55.4(143 / 258)$ & $62.4(133 / 213)$ \\
HADS (CVD) & 1.7 & $30.0(100 / 333)$ & $39.1(102 / 261)$ & $46.7(100 / 214)$ \\
HADS & 1.5 & $37.8(126 / 333)$ & $48.3(126 / 261)$ & $53.7(115 / 214)$ \\
(COPD) & & & & \\
\hline
\end{tabular}

Note: HADS CVD: Hospital Anxiety and Depression Scale for cardiovascular patients; HADS COPD: Hospital Anxiety and Depression Scale for chronic obstructive pulmonary disease patients HUI: Health Utilities Index; THI: Tinnitus Handicap Inventory; TQ: Tinnitus Questionnaire; MCID: minimal clinically important difference criteria.

In the absence of existing data for tinnitus patients, participants were classified as 'improved' on HADS if their total score decreased by 1.7 points (reference group cardiovascular patients; Lemay, Tulloch, Pipe, \& Reed, 2019) and 1.5 points (reference group chronic obstructive pulmonary disease patients; Puhan, Frey, Büchi, \& Schünemann, 2008).Sources for MCID cut-off by outcome: HUI (Horsman et al., 2003; Marra et al., 2005); THI (Zeman, Koller, Schecklmann, Langguth, \& Landgrebe, 2012); TQ (Hall, Mehta, \& Argstatter, 2018);HADS reported for chronic conditions such as cardiovascular disease (1.7 points) (Lemay, Tulloch, Pipe, \& Reed, 2019) and chronic obstructive pulmonary disease patients (1.5 points) (Puhan, Frey, Büchi, \& Schünemann, 2008).

\section{Discussion}

This pragmatic uncontrolled study collected data at four time points over a 12-month period, from 403 participants undertaking a specialised CBT intervention for tinnitusrelated distress. Participants had been suffering from tinnitus for over 4.5 years on average and were all assigned to step 1 of the intervention. Based on eligibility criteria for entry into step 2 of CBT for tinnitus which mirrored (as closely as possible) the treatment as reported previously (Cima et al., 2012), 171 participants also received step 2 over the course of 12 weeks ( $n=25<$ criteria of baseline TQ score of $47 \geq n=146$ ). Results from multilevel modelling indicated that on average, participants' scores on all outcomes showed improvement over a 12-month period. Given that few inclusion and exclusion criteria were applied, the results from this study could be generalized to other contexts or at least similarly resourced healthcare settings and countries.

Analyses revealed that participants younger in age and with lower $\mathrm{THI}$ scores at baseline were more likely to drop out from the study - defined as not completing outcome measures beyond baseline - than other participants. Given that many of these participants completed the audiological assessment and tinnitus information session of step1, they could be better thought of as being less likely to complete outcome measures rather than actually 'dropping out' from the intervention. Furthermore, fewer participants subsequently attended the session with the psychologist, which could indicate that they did not consider their tinnitus a psychological problem or felt some 
stigma at the prospect of seeing a psychologist. Without documentation of the reasons for dropping out though, we can only speculate.

The predictors of step 2 involvement - higher baseline THI, TQ at 3-months, and higher QoL - appear to partially reflect the selection criteria for step 2. Scores between THI and TQ have been found to be highly correlated (Zeman et al., 2012), and it is possible that participants with higher QoL have greater personal resources to be able to 'afford' the time investment required for step 2 of the intervention.

With the exception of HUI-III score at baseline, step 1-only participants reported higher QoL at all time points than participants completing step 1\&2. This suggests that there is further room for improvement for those participants who received both interventions. Nevertheless, the change from baseline to 12-months was greater than established minimal clinically important difference (MCID) criteria for four outcome measures (HUI-III, THI, TQ, HADS) for most of participants (the exception being when a higher cut off was applied for MCID on HADS). Improvement occurred regardless of whether participants received step 1 or step 1\&2 (see Figure 2 and Figures 1 to 6 in SIF 3). Currently, MCID reference data does not exist for the TCS, FTQ and TDI, making it impossible to ascertain whether the observed changes on these measures are meaningful from a clinical perspective.

These results complement those of the RCT of specialised CBT for tinnitus reported by Cima et al. (2012). The samples in the respective studies shared similarities in demographic characteristics (e.g. average age of participants 54 years, and percentage of females $37 \%$ female versus $39 \%$ ), but differed on most patient reported outcome measures at baseline. Specifically, the sample in the current study reported poorer QoL (HUI-III, .53 vs .64), higher mean levels of tinnitus-related interference in daily life (THI, 49 vs 39), tinnitus related distress (TQ, 56 vs 49), tinnitus-related catastrophizing (TCS, 24 vs 21), and tinnitus-related fear (FTQ, 8.3 vs 7.3) at baseline. The sample in Cima et al. (2012) however reported higher levels of psychological distress as measured by the HADS (7.7 vs 12.2). Overall this suggests that the beneficial effects of undertaking specialised stepped care CBT for tinnitus can be achieved in other audiological rehabilitation centres beyond the one in which the original trial was conducted. In other words, the results provide additional "real world" evidence to support the claim that specialised CBT for tinnitus is an effective intervention for reducing tinnitus distress and improving quality of life. This claim is also consistent with Andersson and Hedman (2013) who in a review of effectiveness studies of CBT delivered via the Internet (including for tinnitus patients, though using a different protocol from the one reported here), concluded that "... it is possible to transfer iCBT to clinical practice" (p. 146). The decrease that occurred in THI scores was also comparable to that reported in an effectiveness trial of audiologist-delivered CBT for tinnitus (Aazh \& Moore, 2018).

\section{Limitations and strengths of the study}

Four limitations should be considered in the interpretation and weight given to the results. Firstly, as with any uncontrolled observational study, and thus by definition without randomized allocation of participants to intervention condition, it is not possible 
to draw conclusions relating to the efficacy of the intervention itself. In other words, it is not possible to rule out that the changes between participants' baseline and 12-month scores on the outcome measures were caused by spontaneous recovery, non-specific factors, response shift effects, or other unknown factors. Similarly, without randomized allocation to a control intervention for step 2 after 3 months, it is not possible to comment specifically on the added benefit of step 2 for participants (Van Breukelen, 2006).

A further general limitation related to the design of this study is that it treats and analyses participants at the group level. That is, the results only give an indication of the change that occurred in participants from baseline to 12-months, on average and consequently do not generalize to the participating individuals. An alternative design that could be used in future to examine implementation under real world conditions, but with higher internal validity would be to use Single Case Experimental Designs (Schork, 2015). Single case experimental designs typically require few participants, resources and can relatively easily be incorporated into routine clinical practice (Onghena, Michiels, Jamshidi, Moeyaert, \& Van den Noortgate, 2018; Vlaeyen et al., in press).

Methodological (Podsakoff, MacKenzie, Lee, \& Podsakoff, 2003) and personal biases (Dunning, Heath, \& Suls, 2004) are known to affect measures of health-related outcomes and could also have also had an impact in this study. In particular, the reliance on selfreport questionnaires could be problematic. That is, if participants were optimistic about the benefits of participating in the study, it is possible that this biased the way they responded to the outcome measures. For example, they might have overestimated the benefits of the intervention to align with considerable investment they made in participating in the intervention. Alternatively, if they had high expectations for the intervention that were not met, they might have systematically underestimated the changes/benefits that occurred. The use of independent assessors of outcome (e.g. a psychologist separate from the audiological rehabilitation centre involved in the study) at, for example, 12-months could have generated data that provided an indication of the magnitude and direction of any response bias. Although this limitation applies here, it should be noted that it is also common a limitation of many randomized controlled trials of CBT for tinnitus (Fuller et al., 2020).

Lastly, the fidelity to the protocol with which the intervention was delivered was not taken into consideration in the analysis. Intervention sessions were recorded, but limited resources prevented an unbiased assessment of protocol adherence being undertaken in time to be considered within this study. The absence of protocol fidelity data limits also prevents any assessment of the degree to which sessions might be "contaminated" by intervention components that were not intended to be included in the sessions. Regular meetings with the intervention developer and expert professionals from Adelante throughout the trial were organized to discuss various issues regarding the implementation and to ensure that the intervention was delivered as intended.

Despite these limitations, it should be noted that this study was intended to examine participants' changes over time in a "real world" context, and a randomized controlled trial design was not appropriate for the research question. The sample size included 
here is the largest to date for an implementation study of any CBT protocol for tinnitus and along with the analytical procedures followed, engenders confidence that the results are applicable to a treatment seeking population of tinnitus sufferers in the Netherlands (at least) if not beyond. Furthermore, the steps followed for the analysis mean that we can be confident that we have not overlooked any consistent interactions between the predictors and time or any consistent main effects. MLM analyses are able to include all observed data including of participants with missing outcome data. In our analyses, we also considered the question of whether to include intervention related variables for participants who completed step 2 (e.g. number of sessions in step 2 completed) but ultimately did not do so. This decision was taken on the grounds that any results obtained from such analysis would be difficult to interpret because the number of sessions taken and any other process measure that might have been affected by the outcome as measured at T0 and T1 which in turn correlate strongly with outcome at T2 and T3. This might have produced spurious correlations between process measures and outcome at T2 and T3 if the process measure has no effect on the outcome. For the same reason, an analysis of the full sample, with participation in step 2 (or not) as extra predictor is questionable, as the decision to give a patient step 2 is partly based on that participants' outcome scores at baseline and perceptions of progress/need after completing step 1.

\section{Conclusions}

In sum, data collected at 3-, 8- and 12-months showed, that on average, most participants improved by clinically meaningful amounts compared to baseline scores on all outcome measures regardless of whether they received step 1 only or both steps $1 \& 2$ of the intervention. The observational study design prevents attributions of causality to the intervention itself from being made, but does suggest that specialised CBT for tinnitus as described by Cima et al. (2012) can be implemented at other audiological rehabilitation centres providing that appropriate resources are available. Specialised stepped care CBT for tinnitus is an intervention intended for those suffering from tinnitus (as opposed to people living with tinnitus but not suffering from it), and step 2 in particular for those more severely affected by it. In addition to those of the earlier RCT (Cima et al., 2012), the findings of the current study can be used to inform patients, policy makers, health authorities, and insurance companies in deciding whether to use, fund or promote this promising intervention for reducing tinnitus related distress and improving health related quality of life.

Acknowledgements: We would like to extend our thanks to all the participants and staff at Libra Audiologisch Centrum who contributed to the study. In particular, from Libra, we would like to thank, Petra Karsmakers, Marjo Brans and Guido van den Hombergh who were essential to the recruitment, screening and daily management of the study. 


\section{References}

Aazh, H., \& Moore, B. C. J. (2018). Effectiveness of Audiologist-Delivered Cognitive Behavioral Therapy for Tinnitus and Hyperacusis Rehabilitation: Outcomes for Patients Treated in Routine Practice. Am J Audiol, 27(4), 547-558. doi:10.1044/2018_aja-17-0096

Andersson, G., \& Hedman, E. (2013). Effectiveness of Guided Internet-Based Cognitive Behavior Therapy in Regular Clinical Settings. Verhaltenstherapie, 23(3), 140-148. doi:10.1159/000354779

Baguley, D. M., Humphriss, R. L., \& Hodgson, C. A. (2000). Convergent validity of the tinnitus handicap inventory and the tinnitus questionnaire. J Laryngol Otol, 114(11), 840-843.

Bjelland, I., Dahl, A. A., Haug, T. T., \& Neckelmann, D. (2002). The validity of the Hospital Anxiety and Depression Scale. An updated literature review. J Psychosom Res, 52(2), 69-77. doi:http://dx.doi.org/10.1016/S0022-3999(01)00296-3

Cima, R. F. F., Crombez, G., \& Vlaeyen, J. W. (2011). Catastrophizing and fear of tinnitus predict quality of life in patients with chronic tinnitus. Ear Hear, 32(5), 634-641. doi:10.1097/AUD.0b013e31821106dd

Cima, R. F. F., Maes, I. H., Joore, M. A., Scheyen, D. J., El Refaie, A., Baguley, D. M., . . . Vlaeyen, J. W. (2012). Specialised treatment based on cognitive behaviour therapy versus usual care for tinnitus: a randomised controlled trial. Lancet, 379(9830), 19511959. doi:10.1016/s0140-6736(12)60469-3

Cima, R. F. F., van Breukelen, G., \& Vlaeyen, J. W. S. (2017). Tinnitus-related fear: Mediating the effects of a cognitive behavioural specialised tinnitus treatment. Hear Res. doi:10.1016/j.heares.2017.10.003

Cima, R. F. F., Vlaeyen, J. W., Maes, I. H., Joore, M. A., \& Anteunis, L. J. (2011). Tinnitus interferes with daily life activities: a psychometric examination of the Tinnitus Disability Index. Ear Hear, 32(5), 623-633. doi:10.1097/AUD.0b013e31820dd411

Coyne, J. C., \& van Sonderen, E. (2012a). The Hospital Anxiety and Depression Scale (HADS) is dead, but like Elvis, there will still be citings. J Psychosom Res, 73(1), 77-78. doi:http://dx.doi.org/10.1016/j.jpsychores.2012.04.002

Coyne, J. C., \& van Sonderen, E. (2012b). No further research needed: abandoning the Hospital and Anxiety Depression Scale (HADS). J Psychosom Res, 72(3), 173-174. doi:10.1016/j.jpsychores.2011.12.003

Davis, A., \& El Refaie, A. (2000). Epidemiology of Tinnitus. In R. S. Tyler (Ed.), Handbook of Tinnitus (pp. 1-24). San Diego: Singular thompson Learning.

Dunning, D., Heath, C., \& Suls, J. M. (2004). Flawed Self-Assessment: Implications for Health, Education, and the Workplace. Psychological Science in the Public Interest, 5(3), 69-106. doi:10.1111/j.1529-1006.2004.00018.x 
Feeny, D., Furlong, W., Torrance, G. W., Goldsmith, C. H., Zhu, Z., Depauw, S., . . Boyle, M. (2002). Multiattribute and Single-Attribute Utility Functions for the Health Utilities Index Mark 3 System. Medical Care, 40(2), 113-128.

Folmer, R. L., Theodoroff, S. M., Martin, W. H., \& Shi, Y. (2014). Experimental, controversial, and futuristic treatments for chronic tinnitus. J Am Acad Audiol, 25(1), 106-125. doi:10.3766/jaaa.25.1.7

Fuller, T. E., Cima, R., Langguth, B., Mazurek, B., Vlaeyen, J. W., \& Hoare, D. J. (2020). Cognitive behavioural therapy for tinnitus. Cochrane Database Syst Rev, 1 , Cd012614. doi:10.1002/14651858.CD012614.pub2

Fuller, T. E., Cima, R. F. F., Van den Bussche, E., \& Vlaeyen, J. W. S. (2019). The Fear of Tinnitus Questionnaire: Toward a Reliable and Valid Means of Assessing Fear in Adults with Tinnitus. Ear Hear. doi:10.1097/aud.0000000000000728

Fuller, T. E., Haider, H. F., Kikidis, D., Lapira, A., Mazurek, B., Norena, A., . . Cima, R. F. F. (2017). Different Teams, Same Conclusions? A Systematic Review of Existing Clinical Guidelines for the Assessment and Treatment of Tinnitus in Adults. Frontiers in Psychology, 8(206). doi:10.3389/fpsyg.2017.00206

Gartlehner, G., Hansen, R. A., Nissman, D., Lohr, K. N., \& Carey, T. S. (2006). A simple and valid tool distinguished efficacy from effectiveness studies. J Clin Epidemiol, 59(10), 1040-1048. doi:https://doi.org/10.1016/j.jclinepi.2006.01.011

Grimshaw, J. M., Eccles, M. P., Lavis, J. N., Hill, S. J., \& Squires, J. E. (2012). Knowledgetranslation of research findings. Implementation Science, 7(1), 50. doi:10.1186/1748-5908-7-50

Hall, D. A., Mehta, R. L., \& Argstatter, H. (2018). Interpreting the Tinnitus Questionnaire (German version): what individual differences are clinically important? Int J Audiol, 57(7), 553-557. doi:10.1080/14992027.2018.1442591

Hallam, R. S., Jakes, S. C., \& Hinchcliffe, R. (1988). Cognitive variables in tinnitus annoyance. Br J Clin Psychol, 27(3), 213-222. doi:10.1111/j.2044-8260.1988.tb00778.x

Hoare, D. J., \& Hall, D. (2011). Clinical guidelines and practice: a commentary on the complexity of tinnitus management. Eval Health Prof, 34(4), 413-420. doi:10.1177/0163278710390355

Horsman, J., Furlong, W., Feeny, D., \& Torrance, G. (2003). The Health Utilities Index $\left(\mathrm{HUI}\left({ }_{(}\right)\right)$: concepts, measurement properties and applications. Health and Quality of Life Outcomes, 1, 54-54. doi:10.1186/1477-7525-1-54

Kim, H. J., Lee, H. J., An, S. Y., Sim, S., Park, B., Kim, S. W., . . Choi, H. G. (2015). Analysis of the prevalence and associated risk factors of tinnitus in adults. PLoS ONE, 10(5), e0127578. doi:10.1371/journal.pone.0127578

Langguth, B. (2012). Tinnitus: the end of therapeutic nihilism. The Lancet, 379(9830), 1926-1928. doi:http://dx.doi.org/10.1016/S0140-6736(12)60561-3 
Lemay, K. R., Tulloch, H. E., Pipe, A. L., \& Reed, J. L. (2019). Establishing the Minimal Clinically Important Difference for the Hospital Anxiety and Depression Scale in Patients With Cardiovascular Disease. Journal of cardiopulmonary rehabilitation and prevention, 39(6), E6-E11. doi:10.1097/HCR.0000000000000379

Maes, I. H., Joore, M. A., Cima, R. F., Vlaeyen, J. W., \& Anteunis, L. J. (2011). Assessment of health state in patients with tinnitus: a comparison of the EQ-5D and HUI mark III. Ear Hear, 32(4), 428-435. doi:10.1097/AUD.0b013e3181fdf09f

Marra, C. A., Woolcott, J. C., Kopec, J. A., Shojania, K., Offer, R., Brazier, J. E., . . . Anis, A. H. (2005). A comparison of generic, indirect utility measures (the HUI2, HUI3, SF-6D, and the EQ-5D) and disease-specific instruments (the RAQoL and the $\mathrm{HAQ}$ ) in rheumatoid arthritis. Social science \& medicine (1982), 60(7), 1571-1582. doi:10.1016/j.socscimed.2004.08.034

Newman, C. W., Jacobson, G. P., \& Spitzer, J. B. (1996). Development of the tinnitus handicap inventory. Archives of Otolaryngology-Head \& Neck Surgery, 122(2), 143148. doi:10.1001/archotol.1996.01890140029007

Newman, C. W., Sandridge, S. A., \& Jacobson, G. P. (1998). Psychometric adequacy of the Tinnitus Handicap Inventory (THI) for evaluating treatment outcome. J Am Acad Audiol, 9(2), 153-160.

Onghena, P., Michiels, B., Jamshidi, L., Moeyaert, M., \& Van den Noortgate, W. (2018). One by One: Accumulating Evidence by using Meta-Analytical Procedures for Single-Case Experiments. Brain Impairment, 19(1), 33-58. doi:10.1017/BrImp.2017.25

Podsakoff, P. M., MacKenzie, S. B., Lee, J.-Y., \& Podsakoff, N. P. (2003). Common method biases in behavioral research: A critical review of the literature and recommended remedies. Journal of Applied Psychology, 88(5), 879-903. doi:10.1037/00219010.88.5.879

Puhan, M. A., Frey, M., Büchi, S., \& Schünemann, H. J. (2008). The minimal important difference of the hospital anxiety and depression scale in patients with chronic obstructive pulmonary disease. Health and Quality of Life Outcomes, 6, 46-46. doi:10.1186/1477-7525-6-46

Schork, N. J. (2015). Personalized medicine: Time for one-person trials. Nature, 520(7549), 609-611. doi:10.1038/520609a

Sox, H. C., \& Lewis, R. J. (2016). Pragmatic Trials: Practical Answers to "Real World" Questions. JAMA, 316(11), 1205-1206. doi:10.1001/jama.2016.11409

Sullivan, M. J. L., Bishop, S. R., \& Pivik, J. (1995). The Pain Catastrophizing Scale: Development and validation. Psychological Assessment, 7(4), 524-532. doi:10.1037/1040-3590.7.4.524

Thorpe, K. E., Zwarenstein, M., Oxman, A. D., Treweek, S., Furberg, C. D., Altman, D. G., . . Chalkidou, K. (2009). A pragmatic-explanatory continuum indicator summary (PRECIS): a tool to help trial designers. J Clin Epidemiol, 62(5), 464-475. doi:https:// doi.org/10.1016/j.jclinepi.2008.12.011 
Van Breukelen, G. J. (2006). ANCOVA versus change from baseline: more power in randomized studies, more bias in nonrandomized studies [corrected]. J Clin Epidemiol, 59(9), 920-925. doi:10.1016/j.jclinepi.2006.02.007

Verbeke, G., \& Molenberghs, G. (2000). Linear Mixed Models for Longitudinal Data. New York: Springer-Verlag.

Vlaeyen, J., Wicksell, R., Simons, L., Gentili, C., De, T., Tate, R., . . Linton, S. (in press). From Boulder to Stockholm in 70 years: Single case experimental designs in clinical research. The Psychological record.

Zeman, F., Koller, M., Schecklmann, M., Langguth, B., \& Landgrebe, M. (2012). Tinnitus assessment by means of standardized self-report questionnaires: Psychometric properties of the Tinnitus Questionnaire (TQ), the Tinnitus Handicap Inventory (THI), and their short versions in an international and multi-lingual sample. Health and Quality of Life Outcomes, 10, 128-128. doi:10.1186/1477-7525-10-128

Zigmond, A. S., \& Snaith, R. P. (1983). The hospital anxiety and depression scale. Acta Psychiatr Scand, 67(6), 361-370. 


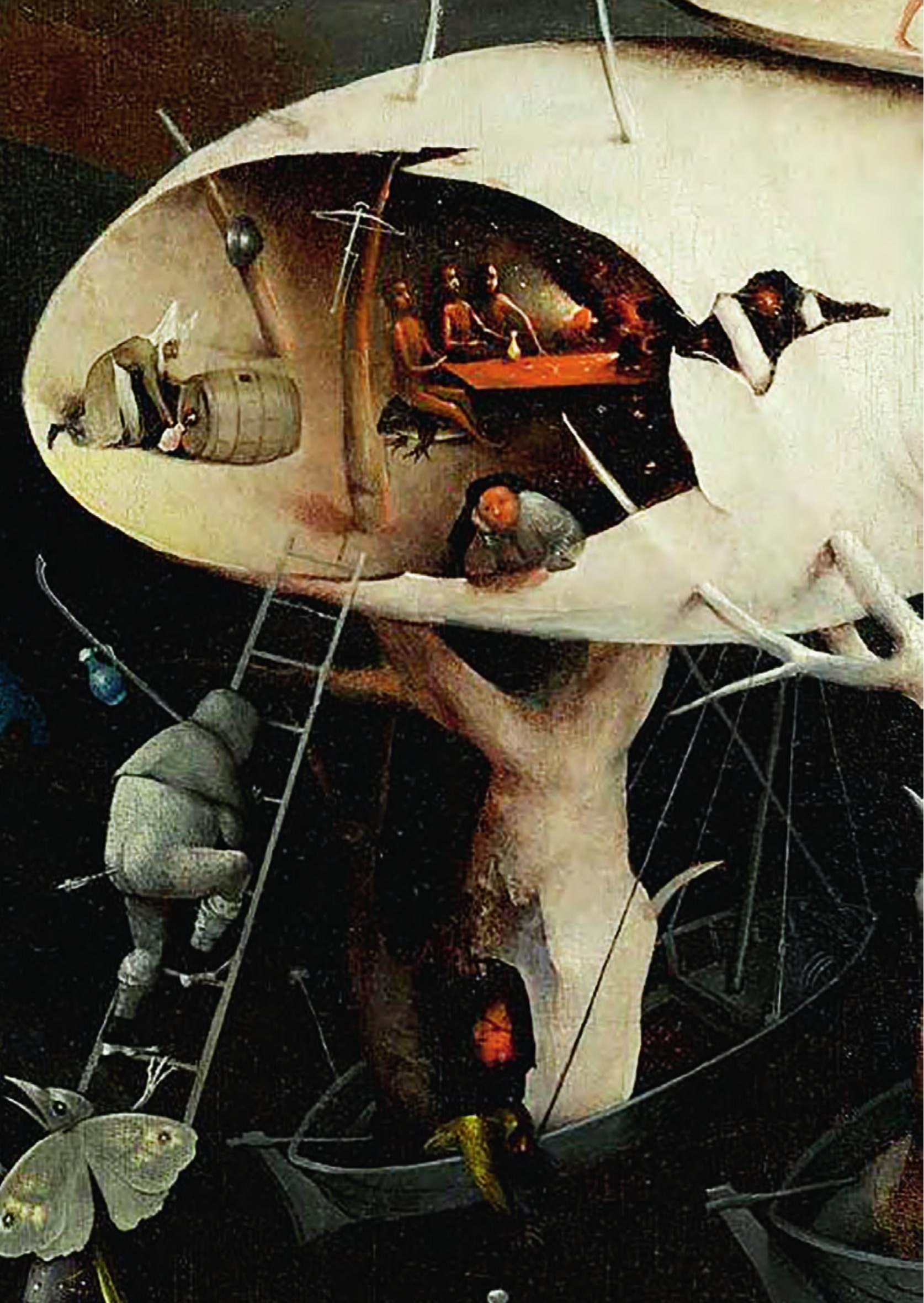




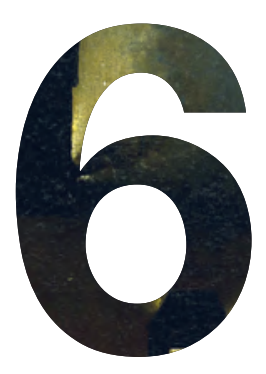

DISCUSSION 
This dissertation has addressed four major research questions. After a general introduction in chapter 1 , we aimed to provide a state of the art review of clinical guidelines on assessment and treatment of tinnitus in chapter 2. Second, in chapter 3 we reviewed existing evidence from randomised controlled trials on the efficacy of Cognitive Behavioural Therapy (CBT) for Tinnitus. Third, in chapter 4 we examined the psychometric properties of a measure developed to assess fear of tinnitus (i.e., the Fear of Tinnitus Questionnaire) since fear and avoidance behaviours are predicted to be key factors in the onset and maintenance of tinnitus suffering. Finally, in chapter 5 we investigated whether a novel specialised stepped-care CBT treatment for tinnitus can be implemented in everyday clinical practice. Here, we present a summary and general discussion of the major implications from the studies that addressed these questions. We also discuss the major limitations and propose research questions to be addressed in the future.

\section{Summary of findings}

To gain insight into the state of the art of existing clinical consensus and recommendations about assessment and treatment of disabling tinnitus in adults, a systematic review of clinical guidelines was performed and presented. As we expected sources to be scarce, we did not specify language or date limits on the literature search and ultimately included clinical guidelines from Denmark, Germany, Sweden, the Netherlands, and the United States in the review. We found that there was a high level of consistency between the guidelines with regard to the recommendations for assessment and treatment of tinnitus. Generally, the guidelines had similar principles in common but differed slightly on the specific methods or treatments recommended. For example, there was consistency in the recommendations for: audiometric assessment; physical examination; use of a validated questionnaire to assess tinnitus-related distress; and, referral to a psychologist when deemed required. Similarly, recommendations for CBT for individuals with disabling tinnitus, the use of hearing aids in instances of hearing loss, and recommendations against the use of medicines were also consistent across the included guidelines. There were however differences between the guidelines regarding recommending, or not, the use of imaging in assessment procedures and sound therapy as a form of treatment for tinnitus distress.

As the systematic review of clinical guidelines showed a consensus among existing guidelines recommending CBT to decrease suffering related to tinnitus, we subsequently addressed the question of whether or not CBT for tinnitus is effective and safe. In collaboration with Cochrane Ear Nose and Throat, we conducted a systematic review and meta-analysis of RCTs of CBT for tinnitus. We applied a broad definition of "CBT" (i.e., one including ACT and mindfulness as CBT), and following Cochrane's strict guidelines for the conduct of a systematic review, included 28 trials. Twenty-three of these trials supplied data for the purposes of meta-analyses from which we concluded that CBT is likely to be safe and superior to: (a) wait-list control conditions/no treatment, (b) usual audiological care, and (c) "other" active experimental control/comparison treatments. Since only one trial compared CBT with Tinnitus Retraining Therapy (TRT), we 
concluded that there was insufficient evidence to establish with any degree of certainty how CBT performed relative to TRT. From the included trials it was clear that there was considerable heterogeneity in the methodologies used (e.g., treatment protocols and outcome measures), and that 6- or 12-month follow-up data were scarce.

One of the prerequisites for improving the health care for individuals suffering from disabling tinnitus is the availability of tinnitus-specific assessment instruments. Therefore, we investigated the factor structure, reliability and validity of the Fear of Tinnitus Questionnaire (FTQ, Dutch version; Cima, Crombez, \& Vlaeyen, 2011) in a sample of 588 participants recruited from Dutch patient-association websites on tinnitus and hearing loss. Based on exploratory and Bayesian confirmatory factor analyses, we found that a single- (tinnitus-related fear) and three-factor (fear of future consequences of tinnitus, deterioration in tinnitus and somatic related fears) Fear of Tinnitus models best fitted the data. We suggested that the three-factor model has greater utility for clinicians since its (potential) subscales reflect clinically relevant issues to address prior to treatment or during a CBT treatment. Furthermore, the analyses demonstrated: (a) that the FTQ had excellent test-retest reliability after a 2-week interval; (b) that FTQ added statistically significant amounts of variance to models predicting tinnitus-related distress and interference in daily life; and, (c) good convergent and concurrent validity. Despite the positive attributes of the FTQ, we recommended that additional research should be conducted to further test and establish norming data and the sensitivity of the measure to patients' changes following treatment. We also concluded that a version with increased response options would be an improvement to the FTQ.

Although the evidence suggests that CBT is safe and superior to other treatments for tinnitus distress, studies testing the effectiveness of CBT for tinnitus in real life contexts are rare. Therefore we conducted an uncontrolled pragmatic study of specialised stepped-care CBT for tinnitus as developed by Cima et al. (2012). The study involved 403 participants with chronic tinnitus. The main aim was to investigate whether the CBT protocol used by Cima et al. (2012) could be implemented under "everyday" conditions. We examined if adults suffering from chronic tinnitus showed improvements in quality of life over a 12-month period, and also examined predictors of favourable outcome. We collected data at baseline, and 3-, 8- and 12-months after baseline, as participants completed either a step 1 only or in case it was deemed clinically necessary, additionally a more intensive step 2. The study was carried out at an audiological rehabilitation centre in Eindhoven, the Netherlands. The primary outcome was health-related quality of life as measured by the Health Utilities Index III (HUI-III) at 12-months. Secondary outcome measures included self-reported levels of tinnitus-related- handicap, disability, affective distress, catastrophizing and fear. We used multilevel modelling (MLM) to examine effects and their predictors. Analyses revealed that, on average, participants improved by clinically meaningful amounts compared to baseline scores on all outcome measures, regardless of whether they received step 1 only, or an additional step 2. The observational study design prevented attributions of causality of improvement/ change in participants to the treatment itself from being made, but did suggest that specialised CBT for tinnitus as described by Cima et al. (2012) can be implemented in other healthcare centres. Furthermore, the findings of this study, in addition to those of 
Cima et al. (2012) and Maes et al. (2014), can be used to inform patients, policy makers, health authorities, and insurance companies in deciding whether to use this treatment for reducing tinnitus-related distress and improving health-related quality of life.

\section{General discussion}

What then do these studies, as a collected body of research, reveal about the assessment, treatment and theories of disabling tinnitus? In brief, they suggest that progress is being made toward standardisation of, or at least consensus on, best practices in the approaches to the assessment and treatment of tinnitus. Chapters 3 and 5 suggest that cognitive behavioural models, in particular the FA model, provide promising explanations for how people suffer from tinnitus. The studies also generated evidence that a CBT protocol based on the FA model of tinnitus works, and that one of the main mechanisms of this model, tinnitus-related fear, can be measured reliably and validly despite some remaining concerns that will need to be addressed. In the following three sections we elaborate on these points and include discussion of the limitations of the scope of the findings.

\section{Assessing tinnitus-related fear and catastrophizing}

Chapters 2 and 4, together reflect that general progress in assessment of the impact of tinnitus is being made in the field. However, discrepancy exists between the methods that are recommended to assess the tinnitus percept, and the impact of tinnitus on daily functioning and quality of life. It is possible that variation at this level results from the availability of numerous questionnaires and methods, and the absence of clear guidelines as to which to use for what purpose. This last point is particularly pertinent when more than one questionnaire is available for assessing the same or similarly defined construct or outcome.

In the context of the FA model for tinnitus, Cima et al. (2011) and Kleinstäuber et al. (2013) foresaw the potential for it to inform assessment, treatment, and to formulate predictions to be tested in fundamental research. Moreover, in relation to assessment, they saw and undertook the development of new instruments to measure the concepts included in the FA model, that is: tinnitus-related catastrophizing to be measured by the Tinnitus Catastrophizing Scale (TCS; Cima et al., 2011); tinnitus-related fear to be measured by the FTQ (Cima et al., 2011); and, avoidance behaviour using the Tinnitus Fear-Avoidance Cognitions and Behaviors Scale (T-FAS; Kleinstäuber et al., 2013). Both the TCS and FTQ were derived from existing pain measures and whereby "pain" was replaced by "tinnitus". This method is sub-optimal, and may weaken face validity, as although there are conceptual similarities between chronic pain and tinnitus, they are not equivalent phenomena. Kleinstäuber et al. (2013) have reported that the T-FAS has moderate internal consistency, is strongly associated with measures of tinnitus-related handicap and weakly correlated with personality traits such as extraversion, openness, agreeableness, conscientiousness and neuroticism. We concluded in chapter 4 that, in its current form, the Fear of Tinnitus Questionnaire is a valid and reliable measure from both a research and clinical perspective, but that it might also be improved with a 
revised response scale. To date, the psychometric properties of the TCS have not been reported. Together, these conclusions suggest that further work refining and analysing the respective measures is required.

Assessing the face and construct validity of the FTQ might have particular importance, as it is possible that the measurement of tinnitus-related fear has not been clearly distinguished from catastrophizing. Given the central role in the FA model that catastrophizing and fear are predicted to play in individuals developing disabling tinnitus, it appears crucial to have these clearly differentiated. As it stands, there appears to be some overlap in the assessment of the respective concepts. To elaborate on this point, we will briefly examine definitions of catastrophizing and fear, and for illustrative purposes, compare one item from the FTQ with items from TCS (Cima et al., 2011) and the cognitions subscale from the T-FAS (Kleinstäuber et al., 2013) respectively.

Firstly, catastrophizing or catastrophic thinking has been defined as:

a tendency to overstate the impact of a condition and to expect major negative consequences ('magnification'), to fear and worry about it ('rumination'), and to feel unable to cope with it ('helplessness') (p. 745; Quartana, Campbell, \& Edwards, 2009).

Beyond tinnitus and pain research, fear has been defined as:

an emotion of anticipation that is triggered when a situation that is at risk for our safety and/or the safety of others is perceived, through either exteroceptive inputs or the endocrine and autonomic nervous systems (interoceptive inputs) (p. 462; Garcia, 2017).

Cima et al. (2011) in the study of the relationship between tinnitus-related catastrophizing and fear, referred to pain-related fear as the: fearful reactions toward pain and painrelated activities and fear of (re)injury, including fearful beliefs about causes of pain (p.634) and by analogy applied this definition to fear of tinnitus. In chapter 4, we defined fear as: an emotional state or response triggered by existing or imminent threats to one's health or safety (p. 1467; Fuller, Cima, Van den Bussche, \& Vlaeyen, 2019). Each of the definitions of fear refer to the emotional response, but it is only the definition of fear by Cima et al. (2011) that is broader in nature and includes "fear-related beliefs" as part of fear. It is the broader scope of the definition that allows for some overlap with both the concept and measurement of catastrophizing and hence potentially weakens the construct and face validity of the FTQ.

To illustrate this confusion we refer here to item 2 on the FTQ: "I am afraid that my tinnitus will become worse" (Table 1). The wording is slightly different from item 6 on the TCS - "I become afraid the tinnitus will get worse" - and the second item on the T-FAS cognitions subscale - "I'm afraid that the tinnitus will become more disturbing and impairing in the long term", but the similarity in meaning is clear. Specifically, in each of the items there is the identification or reference to fear (i.e., "afraid") and the illustration of catastrophic thinking that the condition will get worse or more disturbing. 
Kleinstäuber et al. (2013) do not refer to the T-FAS as a measure of tinnitus-related fear per se, but describes the T-FAS cognitions subscale, as including "catastrophizing cognitions about the consequences of tinnitus deterioration" (p. 93). In addition to this point, a panel of seven people with "clinical and research expertise in tinnitus" recently identified eight items on the FTQ that could best be described as "commonly held erroneous beliefs about tinnitus" (p. 67; Handscomb, 2018). This result adds weight to the general question of what the FTQ is really measuring. Further to this question, a recent study also reported that pain-related catastrophizing measures do not appear to measure catastrophizing as referred to in psychological literature, but instead often measure constructs such as worry and distress respectively (Crombez et al., 2020). A similar method as used by Crombez et al. (2020) might prove insightful with regard to assessing and conceptually disentangling tinnitus-related catastrophizing and fear.

To reiterate, the point here is that the measurement of fear of tinnitus might not have been clearly distinguished from catastrophizing and that the emotional aspect of tinnitusrelated fear overlooked. Future revisions of the FTQ or a new self-report measure of fear of tinnitus that specifically redresses this confusion should be conducted with representatives of likely users of the measures (i.e., patients, healthcare professionals, and researchers) (Mokkink et al., 2019).

While self-report measures of fear are undoubtedly practical, it would also be worthwhile establishing what, if any, relationship scores on the FTQ have to psychophysiological indicators of fear such as skin conductance (Lonsdorf et al., 2017) and (observed) avoidant responses. Early work examining the relationship between self-reported and psychophysiological measures of fear in anxiety disorders indicate it might be weak (Lang, Levin, Miller, \& Kozak, 1983) or even divergent (Hodgson \& Rachman, 1974). While that might be the case, an experimental study examining how healthy participants responded physiologically and with self-reported ratings of emotional valence and arousal during the presentation of pleasant, unpleasant and neutral sounds, found that trait anxiety was associated with physiological reactions (Martin-Soelch, Stöcklin, Dammann, Opwis, \& Seifritz, 2006). What is more, results from repeated exposure to the sounds after a oneweek interval showed that there were different response patterns observed depending on the psychophysiological measure referred to. Specifically, there was not a consistent pattern of habituation or sensitization to the respective sounds across measures of heart rate, skin conductance and muscle activity (Martin-Soelch et al., 2006). 
Table 1. Measures of Tinnitus-related Fear and Catastrophizing.

\begin{tabular}{|c|c|c|c|}
\hline & $\begin{array}{l}\text { Fear of Tinnitus Question- } \\
\text { naire (Cima et al., 2011) }\end{array}$ & $\begin{array}{l}\text { Tinnitus Catastrophizing } \\
\text { Scale (Cima, van Breu- } \\
\text { kelen, \& Vlaeyen, 2017) }\end{array}$ & $\begin{array}{l}\text { Tinnitus-Fear Avoidance } \\
\text { Scale ("Cognitions” subscale) } \\
\text { (Kleinstäuber et al., 2013) }\end{array}$ \\
\hline 1. & $\begin{array}{l}\text { I am afraid that my tinnitus } \\
\text { will deteriorate my hearing }\end{array}$ & $\begin{array}{l}\text { I worry all the time about } \\
\text { whether the tinnitus will } \\
\text { end }\end{array}$ & $\begin{array}{l}\text { I'm afraid that the tinnitus will } \\
\text { threaten/affect my physical } \\
\text { health in the long term. }\end{array}$ \\
\hline 2. & $\begin{array}{l}\text { I am afraid that my tinnitus } \\
\text { will become worse }\end{array}$ & I feel I can't go on & $\begin{array}{l}\text { I'm afraid that the tinnitus will } \\
\text { become more disturbing and } \\
\text { impairing in the long term. }\end{array}$ \\
\hline 3. & $\begin{array}{l}\text { I fear that my tinnitus is the } \\
\text { result of a tumour }\end{array}$ & $\begin{array}{l}\text { It's terrible and I think it's } \\
\text { never going to get any } \\
\text { better }\end{array}$ & $\begin{array}{l}\text { I'm afraid that the tinnitus will } \\
\text { threaten/affect my mental health } \\
\text { in the long term. }\end{array}$ \\
\hline 4. & $\begin{array}{l}\text { Even though my tinnitus is } \\
\text { getting worse, I do not think } \\
\text { it points to a serious disease }\end{array}$ & $\begin{array}{l}\text { It's awful and I feel it } \\
\text { overwhelms me }\end{array}$ & $\begin{array}{l}\text { I'm afraid that the tinnitus will } \\
\text { reduce my quality of life and gen- } \\
\text { eral wellbeing in the long term. }\end{array}$ \\
\hline 5. & $\begin{array}{l}\text { I am afraid that my tinnitus } \\
\text { will drive me crazy }\end{array}$ & $\begin{array}{l}\text { I feel I can't stand it } \\
\text { anymore }\end{array}$ & \\
\hline 6. & $\begin{array}{l}\text { The fact that I have tinnitus } \\
\text { does not mean that my health } \\
\text { is at risk }\end{array}$ & $\begin{array}{l}\text { I become afraid the tin- } \\
\text { nitus will get worse }\end{array}$ & \\
\hline 7. & $\begin{array}{l}\text { I am afraid my tinnitus will } \\
\text { leave me deaf }\end{array}$ & $\begin{array}{l}\text { I keep thinking about } \\
\text { other times I experienced } \\
\text { tinnitus }\end{array}$ & \\
\hline 8. & $\begin{array}{l}\text { I am afraid the moment will } \\
\text { come that my head cannot } \\
\text { withstand tinnitus anymore }\end{array}$ & $\begin{array}{l}\text { I anxiously want the tinni- } \\
\text { tus to go away }\end{array}$ & \\
\hline 9. & $\begin{array}{l}\text { My mental condition will } \\
\text { become severely affected by } \\
\text { my tinnitus }\end{array}$ & $\begin{array}{l}\text { I can't seem to keep it out } \\
\text { of my mind }\end{array}$ & \\
\hline 10. & $\begin{array}{l}\text { I am afraid that tinnitus will } \\
\text { stop me from ever having a } \\
\text { normal life again }\end{array}$ & $\begin{array}{l}\text { I keep thinking about how } \\
\text { strong my tinnitus is }\end{array}$ & \\
\hline 11. & $\begin{array}{l}\text { I am afraid that I will never be } \\
\text { able to experience silence } \\
\text { again because of tinnitus }\end{array}$ & $\begin{array}{l}\text { I keep thinking about how } \\
\text { badly I want the tinnitus } \\
\text { to stop }\end{array}$ & \\
\hline 12. & $\begin{array}{l}\text { I am afraid that loud noises } \\
\text { will aggravate my tinnitus }\end{array}$ & $\begin{array}{l}\text { There is nothing I can do } \\
\text { to reduce the intensity of } \\
\text { the tinnitus }\end{array}$ & \\
\hline 13. & $\begin{array}{l}\text { I am afraid I will not be able } \\
\text { to do anything anymore } \\
\text { because of my tinnitus }\end{array}$ & $\begin{array}{l}\text { I wonder whether some- } \\
\text { thing serious may happen }\end{array}$ & \\
\hline 14. & $\begin{array}{l}\text { It worries me to think I may } \\
\text { never be able to learn how to } \\
\text { cope with this condition }\end{array}$ & & \\
\hline
\end{tabular}


Table 1. Continued.

$\begin{array}{lll}\text { Fear of Tinnitus Question- } & \text { Tinnitus Catastrophizing } & \text { Tinnitus-Fear Avoidance } \\ \text { naire (Cima et al., 2011) } & \begin{array}{l}\text { Scale (Cima, van Breu- } \\ \text { kelen, \& Vlaeyen, 2017) }\end{array} & \begin{array}{l}\text { Scale ("Cognitions" subscale) } \\ \text { (Kleinstäuber et al., 2013) }\end{array}\end{array}$

15. It would be terrible if my tinnitus proved a life-long condition

16. I am concerned that tinnitus may be a risk to my physical health

17. I am afraid that tinnitus may be a preliminary sign of brain haemorrhage or similar

Note: Items in bold were compared in text.

An experimental study by Glombiewski et al. (2015) potentially offers a further illustration of how such an approach to this issue might look for tinnitus suffers. Glombiewski et al. (2015) examined if people scoring highly, compared to people scoring lower, on painrelated fear measures showed distinct patterns of physiological arousal when faced with situations that required them to make feared movements. Adapted to tinnitus, skin conductance rates might be measured and examined when people with high, compared to those with low, levels of tinnitus-related fear are exposed to potentially stressful acoustic environments. If higher FTQ scores were found to predict higher skin conductance, then this would provide some evidence of tinnitus-related fear face-validity.

A third approach that might also provide insights on the validity of self-reported measures of tinnitus-related fear, are expectancy ratings. Expectancy ratings are selfreported indications of how likely a feared outcome is to occur and often take the form of visual analogue scales and are frequently used in fear conditioning research (Boddez et al., 2013). A recent review of the face, diagnostic, predictive and construct validity of expectancy ratings concluded that they are a useful, if also imperfect, research tool for studying fear (Boddez et al., 2013).

This discussion on using the FTQ to measure fear, and for that matter using any standardised self-report questionnaire to measure anything, is based on assumptions that respondents will interpret each item and response option in an identical manner, or as intended by the developers, and that health and experience of treatments are comparable. That is, this represents a nomothetic approach to researching the topic (Sales \& Alves, 2016). Moreover, it also assumes that patients (or study participants) will recognise the relevance and value of completing a particular measure even though this might not be the case (Crawford et al., 2011). An alternative approach to using standardised questionnaires that was developed to redress this limitation, would be to use, for example, the Personal Questionnaire (PQ; Shapiro, 1961). Shapiro (1961) first proposed the $P Q$ as a means for experimental, clinical psychologists to collaboratively develop questionnaires, with and for individual patients. Items would be directly relevant, and had 
response options that were meaningful, to the patient. That is, Shapiro, and others since, proposed the use of idiopathic measures for patients to record outcomes from treatment and processes that occurred during treatment. The structure and scoring of the PQ was standardised to ensure, for example, internal reliability, but the content and meaning of items and responses was determined by each patient (Shapiro, 1961). Idiographic measures such as the $P Q$ are particularly compatible with single-case experimental design studies and effectiveness research (Shapiro, 1961). There are, however, unresolved questions with how best to assess or establish psychometric properties of idiopathic measures as well as to how to compare outcomes between participants.

\section{Progress on treatment of tinnitus distress}

Chapters 2, 3 and 5 have indicated that progress is also being made with regard to the treatment of tinnitus disability. "Progress" in this context could be reflected by: consensus in approaches to managing/treatment of tinnitus; the growing and consistent evidence base for efficacy of CBT for tinnitus; and, a demonstration that implementation of specialised stepped-care CBT for tinnitus is possible under 'real world' conditions.

Our systematic review has revealed that there is broad consensus and (mostly) agreement regarding treatments for tinnitus. [Note: this is not to say that there is coherence and consistency in practice (Cima et al., 2020)]. However consistency in implementation is a separate topic and beyond the scope of this thesis] It is encouraging as well that the authors of the respective guidelines all used an evidence based and transparent approach to the development of the guidelines. While some variation between guidelines or differences in interpretation of the research studies exists, there was wide consistency in regard to identifying which aspects will facilitate patients, clinicians and policy makers in making decisions about treatment choice and service provision. The guidelines also offer directions for new avenues for research and innovation in treatments. Assuming that the methods for creating clinical guidelines remain similar, the recommendations that each guideline makes will also continue to remain consistent and contemporaneous.

The results from our systematic review and meta-analysis of CBT for tinnitus demonstrated that it is likely to be efficacious, and that adverse effects attributable to CBT are extremely rare. This result is consistent with previous systematic reviews on CBT for tinnitus but was reached by following a more rigorous methodological approach. In particular, we excluded some trials that had been included in other systematic reviews as we obtained information from authors of trials that revealed that the studies did not meet current standards for randomisation. Additionally, in our review we examined different forms of CBT independently and, for example, found that the "newer" forms of CBT [i.e., mindfulness and acceptance and commitment therapy (ACT)] are promising. Specifically, in sub-group analyses, albeit with a relatively small number of studies, results indicated no differences in effect sizes between types of CBT were present (Fuller, Cima, et al., 2020). Mindfulness and acceptance-based CBT approaches focus on the responses to challenging situations and responding to them in a functional way. This focus is in contrast to CBT approaches that actively or directly changing the content of the thoughts about the situation that then drive the emotional response (Hofmann \& 
Asmundson, 2008). With regard to the issue of increasing access to tinnitus treatments, Internet-based CBT (iCBT) largely derived from a Swedish treatment manual by Kaldo and Andersson (2004), attained medium effect size on average across studies (as found in sub-group analyses) regardless of the country or language in which it was tested (Fuller, Cima, et al., 2020).

While there are promising results from systematic reviews, Table 2 reflects that there is a scarcity of implementation studies. The table also shows that what is implemented and described as "routine" or "everyday" clinical practice varies significantly between studies. From our pragmatic trial, we concluded that specialised CBT for tinnitus can be successfully implemented in 'everyday' audiological rehabilitation services. While studies evaluating the outcomes of implementation of this approach at additional sites within the Netherlands are required to establish the reliability of this conclusion, it represents a promising foundation for evidence-based routine practice. 


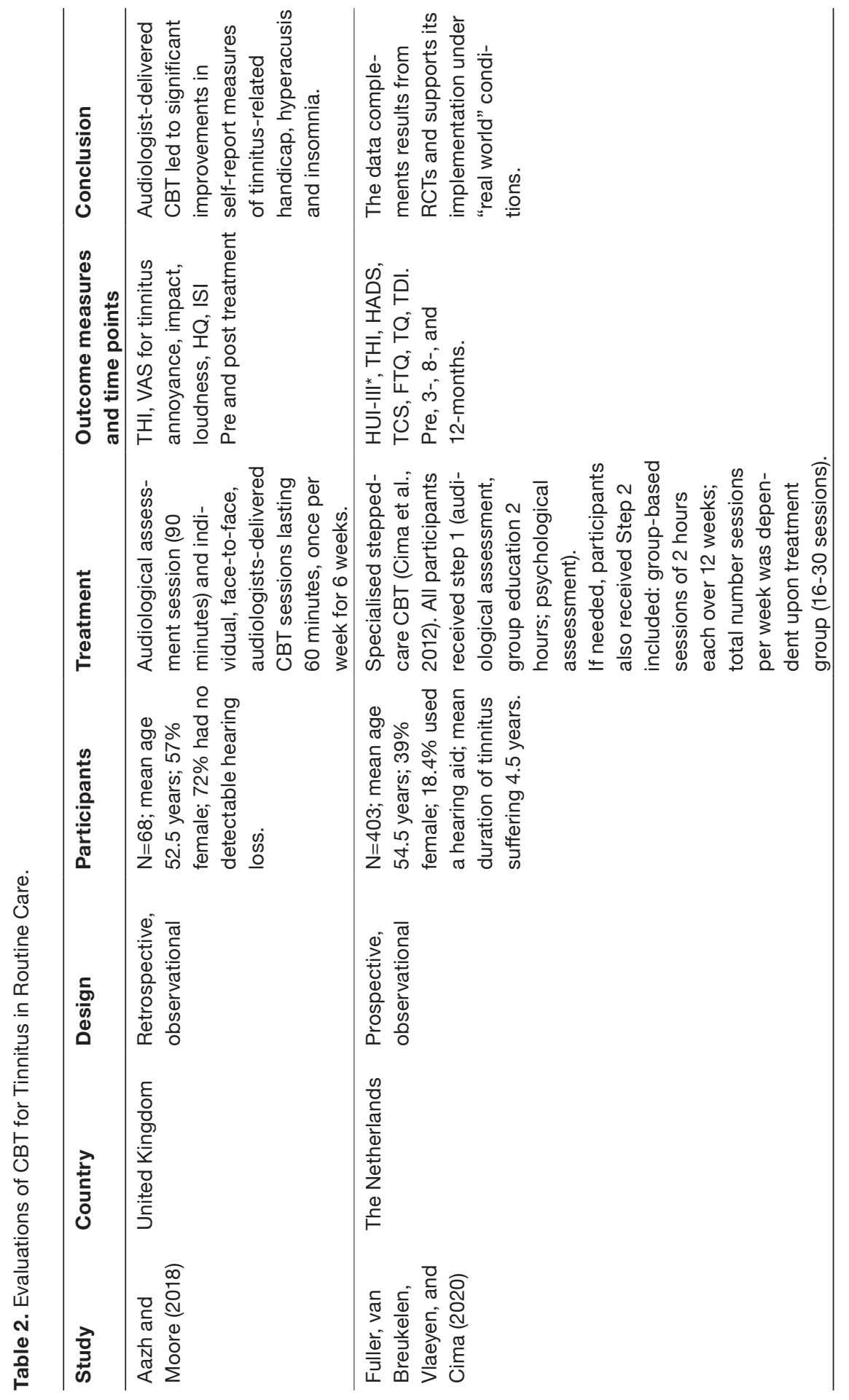




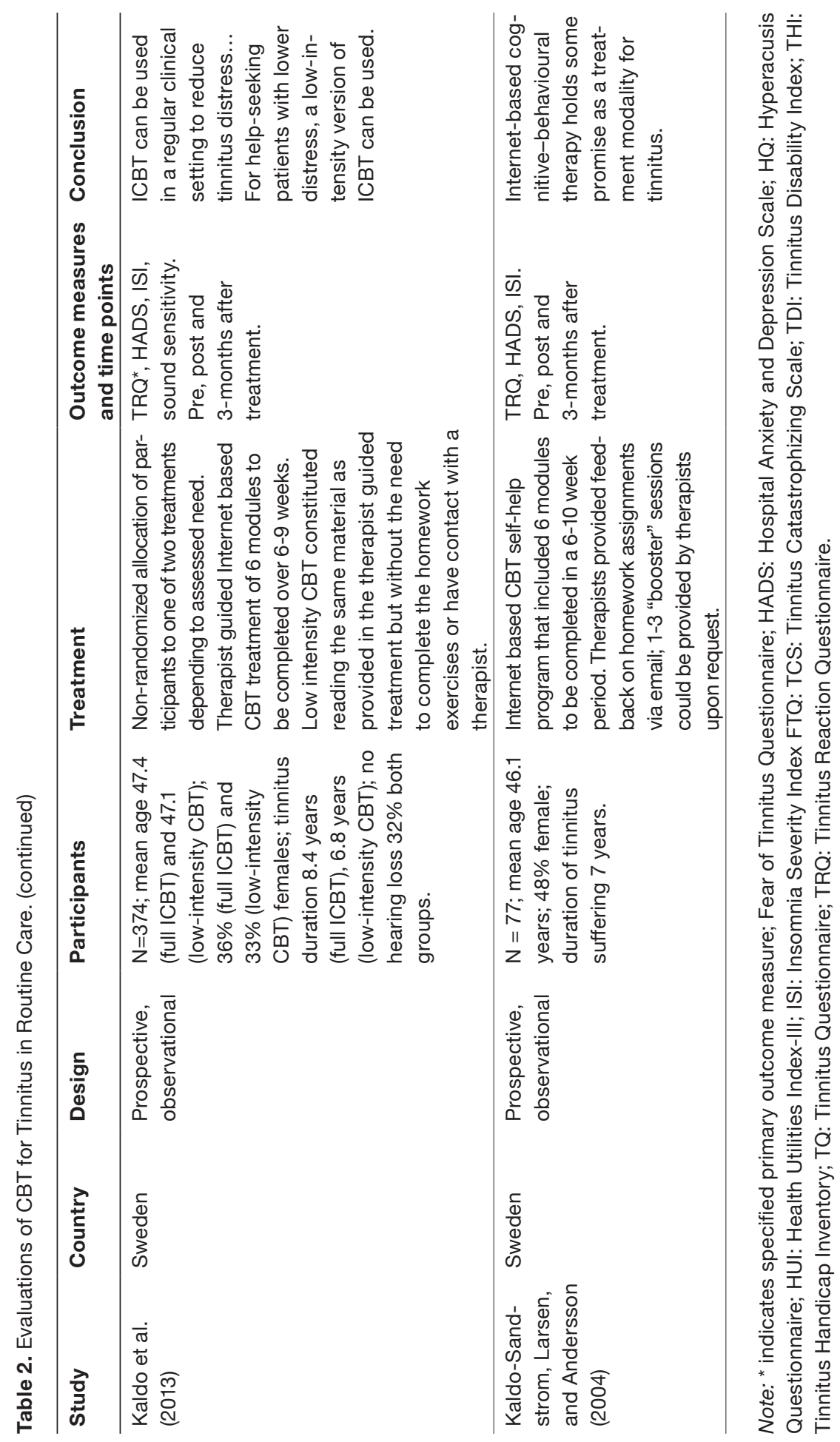




\section{Cognitive behavioural theories of tinnitus-related distress are adequate but should be refined}

Results from the systematic review and meta-analysis of CBT for tinnitus (chapter 3 ) have shown that CBT is, on average, effective, and significantly more so than waiting-list or active control treatments (Fuller, Cima, et al., 2020). Results from our psychometric study (chapter 4), which demonstrated that the FTQ has sound psychometric properties, also complements earlier work showing that improvements in quality of life and decreased levels of distress can be in part attributed to reduced tinnitus-related fear (Cima et al., 2017). Indirectly this evidence has indicated that the cognitive behavioural (CB) and FA models in particular are adequate in providing a foundation for designing and developing treatments to reduce tinnitus-related disability.

Manualised CBT treatment protocols though are not effective for all sufferers, as can be seen by the numbers reported for participants who improved by minimal clinically important differences (MCID; chapter 5). Specifically, 54\% of participants improved according to MCID for quality of life, and $68 \%$ and $62 \%$ according to MCID criteria for tinnitus-related disability as measured by $\mathrm{THI}$ and $\mathrm{TQ}$ respectively. It might be debated as to whether this is a problem of treatment design or implementation, instead of a deficiency associated with the underlying theory from which the treatment was developed. In other words, it might be that individual differences in disabling tinnitus, or particular patient "profiles", account for non-responsiveness or even adverse responses to $\mathrm{CBT}$, rather than there being particular deficiencies in the CBT protocols.

Nevertheless, the possibility that CBT approaches are less than perfect, cannot be dismissed. We discuss what might be needed to refine CBT theories of tinnitus-related distress in the section "next steps for the field" (below).

\section{Limitations of the present research program}

We would like to discuss five limitations specifically related to the research program undertaken for this dissertation and propose how they could be redressed. We will do this before making further, broader suggestions for next steps that the field of tinnitus research could take.

\section{What works for whom?}

Chapters 3 and 5 have indicated that CBT for tinnitus can substantially reduce tinnitusrelated distress. However, the existing studies do not reveal which of the many forms or particular components of CBT might be most effective, or for whom they might be most beneficial. By identifying the most effective and ineffective components respectively, some components could be enhanced while others potentially removed. Possible treatment components that might be relatively more effective could include exposure therapy which specifically targets patients' mis-interpretations of tinnitus and/or their emotional and behavioural (avoidance) responses. This emphasis could potentially lead to treatments becoming more effective and briefer. 
A more accurate understanding of which patient might benefit most from particular components of CBT for tinnitus, could maximise the use of limited resources of patients, clinicians and services alike. For example, for some patients, the social (and social learning) aspect of a group-based treatment might be particularly important but not for others. Treatment preference has rarely been studied in the context of tinnitus. In one study, $46 \%$ of participants, prior to treatment, indicated that they would like to receive a group-based CBT treatment compared with $33 \%$ who had no preference, and $21 \%$ who indicated a preference for an iCBT (Jasper et al., 2014). Interestingly, although participants in the group treatment were more satisfied with the treatment and rated it as more helpful than those who received iCBT, there no statistically significant differences between the groups on the primary outcome measure (tinnitus distress).

Refining CBT treatments and establishing what works for whom requires a shift in focus of researchers from one of simply testing whether a treatment is efficacious or not, to determining what parts of a treatment work and the conditions under which they are effective. Given that the evidence base is growing and largely consistent regarding the efficacy of CBT, researchers could address this question by adopting experimental study designs to examine which theoretically derived components are effective and/or best for whom. Two study designs that might be particularly useful for addressing this are factorial RCTs and single-case experimental design (SCED). Factorial RCTs enable researchers to test the effects of two or more treatments simultaneously and in all possible combinations (Montgomery, Peters, \& Little, 2003; Sedgwick, 2012). SCEDs on the other hand involve "the intensive and prospective study of the individual (or groups of individuals) in which (a) the treatment/s is manipulated in an experimentally controlled manner across a series of discrete phases, and (b) measurement of the behaviour targeted by the treatment is made repeatedly (and, ideally, frequently) throughout all phases" (p. 144; Tate et al., 2016). Both study designs have the potential to examine a range of CBT components, in an efficient method, and with relatively few participants (especially so with SCEDs); with one extrapolating from individuals to a population (SCED), and the other from groups to the population (factorial RCT). Furthermore, both study designs have the potential for high internal validity, as for example, the baseline measurement period, the sequence in which treatment components are delivered, and the start and end points (i.e., duration) of the treatment can be randomised. Even without the use of SCEDs or factorial RCTS, it would be possible, as demonstrated by Glombiewski et al. (2018) in the context of chronic pain, to conduct a RCT in which patient outcomes are compared following randomised allocation to one of three treatments such as: specialised CBT for tinnitus, or to either a brief or long course of exposure therapy. Further studies of this type specifically focussing on tinnitus sufferers, are recommended.

Examining why participants drop out from CBT in tinnitus trials, and when and why relapse might occur, might also inform researchers on what works for whom. For example, it has been found that some participants discontinue a treatment because of a mismatch between treatment and preferences, or because they have already benefited (enough) (Kaldo et al., 2013). Adding 'booster' sessions after treatment has ended, for example, has been suggested as a possible way to reduce the chances of or impact 
of relapse (G. Andersson, Carlbring, Ljótsson, \& Hedman, 2013; Robinson et al., 2008). The effect of booster sessions for tinnitus has not been investigated in the literature, while the evidence for their benefits, depending on the condition under investigation, is varied. For example, E. Andersson et al. (2014) and Wesner, Gomes, Detzel, Guimarães, and Heldt (2015) found booster sessions beneficial when treating obsessive compulsive disorders and panic disorders respectively, but Baker and Wilson (1985) found they did not make a difference to relapse in patients with depression.

\section{Longer-term efficacy of CBT for tinnitus is still unknown}

The relative absence of 6- and 12-month follow-up data from RCTs of CBT for tinnitus is a concern. While many RCTs included a follow-up period, the periods were typically brief, and varied between studies (Fuller, Cima, et al., 2020). The reasons for short follow-up periods (most less than 12 months) were not given, but they do at least have the (potential) benefit, from a methodological point of view, of decreasing the likelihood that participants will have had less time to commence new treatments. If participants do not start a new treatment in the follow-up period, there is at least one less potential confounding variable to consider that prevents attributions of causality being made to the (initial) CBT treatment. How any new treatment introduced in the follow-up period is treated analytically is a question for the individual investigators, but should at least be considered as (another) outcome of the treatment. If the initial treatment had worked well enough, the participant/patient would presumably not have had the need to commence a new treatment.

Bearing this issue of co- (or new) treatment in follow-up in mind, there is evidence from RCTs and meta-analyses that disorder-specific CBT, delivered face-to-face has longterm efficacy for conditions such as: acute stress disorder (Gloster et al., 2013); chronic fatigue syndrome (Deale, Husain, Chalder, \& Wessely, 2001); depression (Cuijpers et al., 2013); and, fibromyalgia (Grossman, Tiefenthaler-Gilmer, Raysz, \& Kesper, 2007). There is also evidence for long-term efficacy of CBT for social anxiety disorder delivered via the internet (Hedman et al., 2011) and CBT for adolescents with anxiety disorders (Kodal et al., 2018). Lastly, in a 5- to 20-year follow-up study, von Brachel et al. (2019) reported that patients from a general mental health care clinic continued to improve from post-treatment to follow-up time points. This evidence suggests that CBT for tinnitus could also have long-term benefits, but without the studies to support such claims, the assertion remains speculative. Failing to collect long-term follow-up data, could also lead to underestimation of (cost) benefit of CBT for tinnitus and provides no indication of what participant relapse rates might be. We could only identify one study that specifically examined relapse following CBT for tinnitus. J. L. Henry and Wilson (1999) reported that all treatment responders (defined as: those who (a) obtained a post treatment score of less than 17 on the Tinnitus Reaction Questionnaire (TRQ); and (b) displayed a reduction in TRQ scores of at least $50 \%$ from pre-treatment to post-treatment) maintained the gains at end-of-treatment at the 12-months follow-up. It should be noted however that: this study (and result) has not been replicated; that there was no control group; and, that $18 \%$ of the initially enrolled participants did not attend at least six of eight treatment sessions and were excluded from the analyses (J. L. Henry \& Wilson, 1999) suggesting that this finding should be interpreted cautiously. 
Overall, the gap in knowledge about the long-term efficacy of CBT for tinnitus could be addressed by ensuring that funding for longer-term follow-up periods is included in grant applications and that participants' response burden at follow-up time points is minimised. Incentives for participants to complete the long-term follow-up measures regardless of whether they have improved or not might also be required to minimise biases in responses; i.e., it might be that participants who fare well in the long-term are more likely to respond than those who do not.

Absence of a control group in the pragmatic trial of specialised CBT for tinnitus The absence of a control group from the pragmatic trial of specialised CBT for tinnitus represents a limitation of the pragmatic study (chapter 5 ). While this limitation also applies to other pragmatic studies of CBT for tinnitus (Table 2), without a control group in this study we cannot discount alternative explanations such as non-specific effects (e.g. treatment expectancies, attention) and reactivity to repeated use of the same questionnaires, for participants improving on outcome measures (Cook \& Campbell, 1979). Spontaneous recovery is an alternative explanation for improvement in participants that appears both possible and improbable. It appears unlikely, on one hand, as participants had suffered from tinnitus for 4.5 years on average and (many) had to wait several months before commencing the treatment. However, it too cannot be ruled out completely as a recent meta-analysis of participants in wait-list control groups showed statistically significant change over the non-treatment period (Phillips, McFerran, Hall, \& Hoare, 2018).

An innovative approach to effectiveness studies that could address the absence of a control group per se, would be to use single-case experimental design methodologies. Doing so, as already mentioned (above), would provide the opportunity to obtain data from participants who act as their own controls, with a high level of internal validity, on the effectiveness of an treatment in real time (Vlaeyen et al., 2020). Lastly, asking participants to complete questionnaires about their expectations and the perceived credibility of the treatment, while awaiting the start of the assessment and/or treatment, might also offer additional insights into moderating factors of treatment outcome (Vlaeyen \& Morley, 2005). It could also help therapists by alerting them to unrealistically high expectations of treatment. For example, if participants expect that CBT for tinnitus will 'cure' them of the disabling effects of their tinnitus, then this unrealistic expectation could easily to be addressed.

\section{Treatment fidelity of specialised CBT for tinnitus when implemented in everyday practice remains unknown}

Assessing fidelity to a manualised protocol is an important aspect of establishing whether a treatment is effective, as it (treatment fidelity) can act as a moderating factor in changes that might occur. Whenever possible, it is recommended that trained, blinded, independent raters assess fidelity by viewing recordings of sessions (Waltman, Sokol, \& Beck, 2017), although it is also recognised that this method is resource intensive (Wiltsey Stirman et al., 2018). As it was, in this study, video and audio recordings of most of the sessions were made, but we did not have the capacity at this time to code the data and include it in the multilevel regression analyses. Without an estimation of treatment 
fidelity, we cannot be sure what clinicians delivered. That is, for example, we do not know if they included treatment components that were not relevant or even "prohibited" for participants from particular sessions (Leeuw, Goossens, de Vet, \& Vlaeyen, 2009).

While we cannot be sure of the treatment fidelity in our pragmatic trial, the methods used by McKenna, Marks, Hallsworth, and Schaette (2017) to ensure treatment fidelity in their study were similar to practices that we used for quality control. Specifically, McKenna et al. (2017) in an RCT of mindfulness-based cognitive therapy for chronic tinnitus, informally assessed and ensured fidelity to the protocol through regular meetings and supervision between clinicians and supervisors. An additional feature in our study that might have ensured fidelity was the provision of detailed, manualised treatment protocols to all participating therapists. Both these methods (i.e. supervisory meeting and manualised protocols) were included in the management of the study but not considered part of the planned assessment of treatment fidelity per se.

\section{Children and adolescents}

While we recognise that a dissertation cannot address everything, we would like to highlight that we have not discussed research relating to tinnitus in children and adolescents. Results from three recent systematic reviews indicate that it is likely to be a common problem (Rosing, Schmidt, Wedderkopp, \& Baguley, 2016), with a range of disabling effects (Smith et al., 2019) and for which there is very little evidence associated with efficacy or effectiveness of treatments (Lee, Lee, \& Kim, 2018). Estimates of prevalence of tinnitus in children with normal hearing range between $4.7 \%$ and $46 \%$; between $23.5 \%$ and $62.2 \%$ in children with some detectable level of hearing loss; and, between .6\% and $49.2 \%$ of children experience disabling tinnitus (Rosing et al., 2016). Rosing et al. (2016) also highlight that the many issues resulting from a lack of a consensus-based definition of tinnitus in adults also affects the estimates of prevalence of tinnitus in children. A complementary systematic review of studies in which the disabling impact of tinnitus in children (i.e., under 18 years) generated six categories that summarised the domains of problems experienced as: physical (e.g., sleep, fatigue, headache); cognitive health (e.g., attention and concentration); hearing and listening difficulties (e.g., at home or school); emotional health (e.g., anxiety, distress, fear); quality of life; and, feeling different/isolated (Smith et al., 2019). Lastly, this review of treatments for treatment of tinnitus in children and adolescents only included three studies (Lee et al., 2018) indicating that research is scarce on this topic.

Longitudinal research studies including samples of children and adolescents with or without tinnitus, might yield valuable clues into factors associated with the onset of acute and or chronic tinnitus and as to why some but not all people develop disabling tinnitus. Alternatively, testing early treatment strategies in children and adolescents after onset of chronic tinnitus might reduce or avoid longer-term negative outcomes, through for example, reducing the chances of catastrophic thoughts about tinnitus. These are but brief proposals to begin to address some of the research and clinical questions that need attention. 


\section{Next steps for the field}

Within this section, we propose five topics that could be addressed by tinnitus researchers in order to drive innovation in theory and clinical practice which in turn could lead to improved outcomes for patients. Specifically, researchers and clinicians could: (a) strive for greater coherence as a field of research; (b) refine the FA and CB models of tinnitus distress; (c) refine CBT for tinnitus; (d) minimise suffering from tinnitus through identification of risk factors; and, (e) increase access to CBT for tinnitus.

\section{Strive for greater coherence as a field of research}

Evidence-based health care needs evidence. It needs evidence firstly of efficacy (it works), then cost-effectiveness (it works better, and the beneficial effects outweigh the possible additional costs, when compared to existing treatments), and implementation in realworld settings (it reaches the individual who may benefit from the treatment) (Heneghan et al., 2017; Sackett, Rosenberg, Gray, Haynes, \& Richardson, 1996). Without evidence, there is only theory, experience and opinion from which patients receive care. Given that there is currently no consensus on an agreed definition of tinnitus, it is not surprising that the trajectory from diagnosis, assessment to treatment is often a fragmented and circuitous experience for patients (Hoare, Gander, Collins, Smith, \& Hall, 2012).

Audiologists, psychologists, ENT surgeons and general practitioners are some of the professionals patients might see. Behind the scenes, biologists, neurologists and psychologists working in science are also investigating the causes and treatment of tinnitus. Diversity of discipline-specific perspectives and methodological approaches is likely to yield more valuable and plentiful insights and innovation than from any one field alone. However, when a field is too fragmented, methods too varied, results and opinions too disparate it is difficult for policy-makers, insurance companies, and most importantly patients to discover the most promising procedure or treatment in a given set of circumstances.

Reaching a consensus on definition(s) would thus represent a significant milestone for the field. It would at least begin to better account for the variety that exists in estimates of prevalence and incidence of tinnitus (McCormack, Edmondson-Jones, Somerset, \& Hall, 2016). In addition to building a shared language between international multidisciplinary research groups, agreement on this issue would facilitate comparison, evidence synthesis and collection of epidemiological data.

Similarly, reaching consensus on establishing a minimum, core set of outcome measures that researchers should include in clinical trials would also be valuable. It would enable a growing body of research to form, from which data could (more) easily be compared and synthesised. The work currently being undertaken by the COMit'ID project (Hall et al., 2015; Hall et al., 2016; Hall et al., 2018) aims to recommend such a core set. Whatever the recommendations for the core set of outcome measures from the COMit'ID project, success (i.e., use of the minimum set of measures) will be largely dependent on uptake by researchers. 


\section{Refine the Fear Avoidance and Cognitive Behavioural models of tinnitus- related distress}

We briefly argued (above) that there is evidence that the FA and CB models of tinnitus distress have validity and value in acting as the foundation for cognitive behavioural treatments but that is likely that they should be refined. The main way for this to occur is through focussed and systematic investigation of the components described/contained within the models, and by testing the hypotheses that might be generated by/derived from the models. Triangulation and replication of results could be attempted by using a range of methodologies (i.e., quantitative, qualitative, experimental, longitudinal), and by including participants without tinnitus (for example to potentially act as "healthy" controls, or participants in experimental studies), participants with tinnitus but not suffering from it, and those who suffer from it to varying degrees. Some of this work has already been conducted (e.g., Handscomb, Shorter, Hoare, \& Hall, 2019) but more is required.

By way of an example, two studies have recently examined the CB model of tinnitus distress (McKenna, Handscomb, Hoare, \& Hall, 2014). The first comprised a crosssectional survey completed by 342 people who suffered from tinnitus (Handscomb et al., 2019). The factor structure of the surveys was assessed and the subsequent results used in path analyses to test the CB model. While the model was considered a good fit for the data, two versions were considered appropriate (Handscomb et al., 2019). The overall validity, robustness, and reliability of the conclusions from this study was fundamentally dependent on the choice and administration of questionnaires used. With that in mind, we note that some of the questionnaires were not used in their original format (i.e., it used two subscales from the Illness perception questionnaire that were extracted from the full version), which might undermine the replicability of the findings/ conclusions. The second study comprised interviews with 11 tinnitus patients and focus groups with five therapists to assess the face validity of the model (Handscomb, 2018). From the ensuing thematic analysis of the qualitative data, Handscomb concluded that the model was, 'broadly' a good fit with patients' experience, although some participants did not identify with all the components in the model. The results from both studies suggest that the CB model in its entire form might not be relevant for all tinnitus sufferers although it is not clear which participant characteristics might affect the perceived personal relevance of the model.

In relation to examining the FA model of tinnitus, catastrophizing, fear, and avoidance have all received some attention (e.g. G. Andersson, Juris, Classon, Fredrikson, \& Furmark, 2006; G. Andersson, Kaldo, Strömgren, \& Ström, 2004; Cima et al., 2011; Kleinstäuber et al., 2013). Of these, catastrophizing and tinnitus-related fear have been found to be related to tinnitus distress (Cima et al., 2011) and that decreases in tinnitusrelated fear, as measured with the FTQ account for improvements in quality of life following stepped-care CBT (Cima et al., 2017), which was not the case in the usual care control group. [Note though that, for example, just because a questionnaire is good at predicting or accounting for change in a patient's state, does not necessarily mean that it has good content or criterion validity (Crombez et al., 2020).] Tinnitusrelated avoidance behaviours (and measures thereof) however have been studied less 
often and not with the a priori purpose of testing psychological models of tinnitusrelated distress. For example, the study by G. Andersson et al. (2006) reported that focussing on tinnitus resulted in an increase in tinnitus-related thoughts whereas cognitive avoidance strategies reduced tinnitus-related thoughts, neural activity in the auditory cortex, and perceived loudness and annoyance. Even though findings seem consistent with theoretically derived predictions that exposure is stressful/challenging, and avoidance helpful in the short term, these do not yield information about the longerterm effects predicted by the FA model. The FA model predicts that these short-term improvements serve as reinforcement of safety-behaviours, to the detriment of the longer term outcomes.

Thus, overall, there is evidence for at least parts of psychological models of tinnitus distress. A better understanding of, for example, tinnitus-related avoidance behaviour(s) is required and, given that vast heterogeneity exists between tinnitus patients, it might be the case that more than one empirically validated model of tinnitus distress is required to adequately account for the variation. Answers to the following research questions will strengthen the foundation of models of tinnitus distress:

- Why some people fear tinnitus, and which vulnerability (e.g., negative affect, intolerance of uncertainty) and resilience factors (e.g., positive affect, optimism) contribute to or protect against chronic disabling tinnitus?

- Given the lack of the long-term follow-up data, what proportion of people relapse following CBT? Further, and assuming there is some relapse, what role might a "return of fear" (to borrow a concept from pain and anxiety disorder research) have in relapse?

- Do high levels of tinnitus-related fear predict the use of safety and avoidance behaviours in tinnitus patients?

Here we discuss two additional approaches for refining the FA and CB models of tinnitus distress. One includes considering transdiagnostic factors into the models and the other, focusses specifically on tinnitus-related fear.

First, consideration could be given to investigating or including in a revised version of the FA or CB models of tinnitus, transdiagnostic vulnerability factors such as intolerance of uncertainty. Intolerance of uncertainty refers to "difficulty enduring the experience of not knowing" (p. 438; Rosser, 2019). It is has been found that individuals high in intolerance of uncertainty use cognitive, emotional and behavioural approach or avoidant strategies to create more certainty for themselves when in potentially threatening situations (Birrell, Meares, Wilkinson, \& Freeston, 2011). Although intolerance of uncertainty has not been investigated within tinnitus patients, it has been investigated in two closely related domains. For example, within pain research, two recent studies have examined intolerance of uncertainty, pain perception and levels of functioning. In an experimental laboratory-based study with "healthy" participants higher intolerance of uncertainty scores were associated with increased pain perception (Bélanger et al., 2017); and in a cross-sectional study with chronic pain patients, higher intolerance of uncertainty scores were associated with poorer functioning (Fischerauer, Talaei-Khoei, 
Vissers, Chen, \& Vranceanu, 2018). Patients with Ménières disease (an audiological disorder with symptoms that often include tinnitus) with higher levels of intolerance of uncertainty have also been found to have higher levels of anxiety (Kirby \& Yardley, 2009). Intolerance of uncertainty could thus also have particular relevance for people whose primary complaint is tinnitus since it might be associated with increased reactivity to situations perceived as threatening (Bauer et al., 2020). For tinnitus sufferers, for example, leaving the safety of home, where they have control over their immediate environment to go outside where they have less control, might be enough to increase arousal and increase the likelihood of interpreting sounds as more threatening and likely to lead to a deterioration of their tinnitus. Moreover, since it is not known when, why or how acute tinnitus becomes disabling, tinnitus itself could be viewed as a symptom with an uncertain course. In sum, although there is evidence from related fields for the potential utility of "intolerance of uncertainty", experimental and cross-sectional studies (for example) are required to establish its role within FA and/or CB models of tinnitus distress.

A second approach could be to re-examine the tinnitus-related fear and catastrophizing constructs and how these are conceptualized, defined, measured and validated. Reexamination and new insights into these constructs by themselves and their possible conceptual overlap could strengthen the FA model (if not also be relevant for the CB model). Refining the assessment of tinnitus-related fear could occur in at least two ways. First, consideration could be given to revising the wording of items in an effort to discriminate tinnitus-related fear from tinnitus-related catastrophizing. The following suggestions provide examples to this point which was first raised earlier in this chapter. Specifically, disentangling catastrophizing from tinnitus-related fear might be achieved by removing reference to any beliefs or cognitions about the consequences of being exposed to fear inducing situations. For example, I feel afraid when I hear loud music. Alternatively, tinnitus sufferers could be asked to identify their physical signs when afraid, and then could subsequently use this response in the context of a statement such as: I feel (physiological indication of fear) when I hear loud music. A second or complementary approach to strengthening the criterion and construct validity of the FTQ, as discussed earlier, might also be to examine how scores on the FTQ are associated with psychophysiological measures of fear in tinnitus sufferers. Finally, three additional steps could be taken to generate additional evidence for and means to further test important components of the FA model of tinnitus. They are to: validate the English version of the FTQ; report the psychometric properties of the TCS; and, develop measure of tinnitus-specific avoidance behaviours.

\section{Improve the impact of CBT for tinnitus}

Conclusions from systematic reviews and meta-analyses of CBT for tinnitus consistently revealed that CBT appears to be efficacious and the best currently available empirically supported treatment for decreasing tinnitus-related distress and interference in daily activities (Aazh, Landgrebe, Danesh, \& Moore, 2019; Cima, Andersson, Schmidt, \& Henry, 2014; Fuller, Cima, et al., 2020; Hesser, Weise, Westin, \& Andersson, 2011; Hoare, Kowalkowski, Kang, \& Hall, 2011; Mehta, Peynenburg, \& Hadjistavropoulos, 2019; Rademaker, Stegeman, Ho-Kang-You, Stokroos, \& Smit, 2019). While that might 
be the case, effect sizes are typically modest (on average) for CBT for tinnitus (Fuller, Cima, et al., 2020), and the treatments, if they are available at all, are usually demanding on patients' resources.

In order to address the issue of how to improve and refine CBT for tinnitus, four strategies are required. Firstly, as we commented on in chapter 3 , there is a scarcity of CBT treatment protocols available. This obscures what is actually included within any one specific treatment. This obscurity is problematic for practitioners of CBT for tinnitus (and any other condition) where there are many components and varied combinations of treatment components that could be used. Thus, if researchers and clinicians cannot clearly establish what is contained within a treatment in a given study, then developing hypotheses for determining what might be most effective for whom becomes increasingly challenging. Furthermore, this lack of information about the contents of an treatment (akin to a therapeutic black box) adversely affects assessments of heterogeneity between studies, and the ability for other researchers groups to attempt to replicate studies or test the treatments under different conditions.

A complementary approach to improving benefits for tinnitus patients could involve testing the utility of brief questionnaires such as the Suitability for Short-term Cognitive Therapy Scale (Myhr, Talbot, Annable, \& Pinard, 2007; Renaud, Russell, \& Myhr, 2014) or the CBT Suitability Scale (McLellan, Peters, \& Rapee, 2016) to determine if patients are likely to also benefit from CBT for tinnitus. These measures have previously been tested with people living with anxiety and depression (Renaud et al., 2014) and social phobia (McLellan, Stapinski, \& Peters, 2019) and found to be predictive of various outcome measures. The measures might be effective as the items (e.g., I can change the way I feel about things by changing the way I think about them) appear to be closely related to the core principles of CBT. While such questionnaires might not directly improve outcomes for all tinnitus patients, they could potentially prevent people suffering from tinnitus investing time and money into a treatment that they might be unlikely to benefit from. This would also have the flow-on effect of creating places for patients who might be more likely to benefit.

A further, consideration to potentially improve outcomes of CBT for tinnitus pertains to participant sample characteristics. Specifically, minority groups are often overlooked in recruitment for CBT trials (Hofmann, Asnaani, Vonk, Sawyer, \& Fang, 2012). Without representative samples and/or treatments tailored to minority groups, conclusions from clinical trials (and especially observational studies) are unlikely to be generalizable. For example, in an RCT of CBT for post-traumatic stress disorder (PTSD) $45 \%$ of African Americans compared with $73 \%$ of Caucasians completed treatment (Lester, Resick, Young-Xu, \& Artz, 2010). Given that, CBT treatment protocols should be tailored to minority groups to maximise the chance of achieving therapeutic benefit (Hinton, Rivera, Hofmann, Barlow, \& Otto, 2012).

A fourth approach is to extend experimental laboratory-based studies of tinnitus-related phenomena, since they can potentially improve insights into the working mechanisms underlying the onset, maintenance and manipulation of tinnitus. They can, of course, 
also be used to manipulate and test the variables included in the respective models [see for example, the study conducted by G. Andersson et al. (2006) which manipulated the use of suppressing thoughts about tinnitus].

\section{Minimise suffering from tinnitus through identification of risk factors}

Identifying modifiable risk factors following the onset of tinnitus could prevent or minimise the impact of disabling tinnitus (i.e. secondary prevention). Possible candidates from chronic pain research that have been identified as having a high level of evidence for predicting disability (Main, Kendall, \& Hasenbring, 2012) that might warrant further investigation within tinnitus research include:

- Dysfunctional beliefs about tinnitus and avoidance behaviour.

- Pre-existing negative affect might make it more likely that tinnitus is perceived negatively, while also having diminished ability to reduce its interference in daily life activities.

- The role that operant factors such as rewards and punishments play in developing and/or maintaining disabling tinnitus. For example, it might be that disability or insurance payments are related to the ongoing (demonstration of) impact from tinnitus.

- Early retirement, redundancy, as well as unemployment or the duration of absence from employment.

It is worth noting that in a systematic review and meta-analysis of screening questionnaires with prognostic value for chronic pain patients, that measures such as the Örebro Musculoskeletal Pain Questionnaire were better at predicting disability rather than pain intensity in patients with recent onset low back pain (Karran et al., 2017). This finding could have parallels with risk factors for disabling tinnitus, especially given that perceived loudness of tinnitus is only weakly associated with levels of tinnitus annoyance (G. Andersson, 2003; Hiller \& Goebel, 2006), distress and interference in daily life activities (Cima et al., 2011).

Additional risk factors shown to have prognostic value in the context of negative affect that might also have relevance for tinnitus patients could include transdiagnostic factors such as a propensity for rumination and intolerance of uncertainty (discussed above). For example, in two longitudinal studies, rumination has been found to mediate predicted increases in anxiety in adolescents, and anxiety and depression in adults (McLaughlin \& Nolen-Hoeksema, 2011). Rumination, thus might also have some predictive value for people with tinnitus.

\section{Increase access to CBT for tinnitus}

Access to specialist tinnitus rehabilitation services is limited and patients are dependent on the available services. In turn, services are dependent on funding and infrastructure that might change with governments' health policy decisions. Within the EU alone there is great variety in opinion of best practice and the provision of assessment and treatment services (Cima et al., 2020). We propose two approaches to increasing access to CBT for tinnitus. 
Firstly, increasing access to CBT treatment could be through the funding of iCBT services. Internet-based CBT offers the potential for standardised, evidence-based treatment to be disseminated widely. Sub-group analyses in chapter 3 indicated that iCBT for tinnitus was effective (Fuller, Cima, et al., 2020) indicating that the fundamental work has been completed. Unfortunately, iCBT for tinnitus is usually only available in the context of research studies and ongoing funding is either not pursued, or obtained. An unresolved issue also remains as to what service delivery model of iCBT for tinnitus might be best. For example, iCBT for tinnitus could be used as part of a steppedcare model of service provision, integrated with face-to-face therapy or delivered as a stand-alone treatment (Beukes, Allen, Manchaiah, Baguley, \& Andersson, 2017; Beukes, Baguley, Allen, Manchaiah, \& Andersson, 2018; Beukes, Manchaiah, Allen, Baguley, \& Andersson, 2019). There are also some cautionary results from cost-effectiveness studies of iCBT for other chronic conditions. For example, for depression some studies conclude that it is cost-effective (Baumann, Stargardt, \& Frey, 2020; Kraepelien et al., 2018), while others do not (Kenter et al., 2015).

Another approach to increasing access to CBT for people suffering from tinnitus could focus on increasing the numbers of professionals interested in and qualified to deliver the treatment. This could be achieved through, for example, registered/licensed psychologists who already deliver CBT treatments receiving additional tinnitus specific education and training. Alternatively, and/or additionally, testing different durations and, for example, effectiveness of group- compared to individual-audiologist delivered CBT (Aazh \& Moore, 2018; Beukes et al., 2018) would be informative. Lastly, refining and investigating stepped-care models of treatment delivery could lead to efficient allocation of scarce resources/services (e.g., Cima et al., 2012; J. A. Henry et al., 2017). 


\section{Conclusions}

From the studies included in this dissertation we can draw four main conclusions. First, that each of the groups involved in the development of clinical guidelines are committed to the principles of evidence-based healthcare and consequently make recommendations for the assessment and treatment of tinnitus that are largely consistent with each other. Second that CBT is likely to be an effective and safe treatment for most people who use it, and likely to be superior to other currently available treatments such as relaxation, education, or peer support. The longer-term efficacy, however, is yet to be established. Thirdly, that the measurement of tinnitus-related fear, as a concept of the FA model of tinnitus, is in the early stages of development. The FTQ, though likely to benefit from revision and further validation, has demonstrated sound psychometric properties and that it is useful in clinical or research in settings. Finally, that specialised CBT for tinnitus is a promising treatment that can be implemented into everyday practice.

Together the studies also demonstrate how research and clinical practice can inform each other to expand the evidence base from which patients and policy-makers can make informed choices about service use, supply and development. The studies highlight the promising foundation from which the theory and treatment of disabling tinnitus can continue to build. It is an imperative, though, that the longer-term efficacy of CBT is examined along with determining what kind or part of CBT works best for whom and under what conditions. 


\section{References}

Aazh, H., Landgrebe, M., Danesh, A. A., \& Moore, B. C. J. (2019). Cognitive Behavioral Therapy For Alleviating The Distress Caused By Tinnitus, Hyperacusis And Misophonia: Current Perspectives. Psychology research and behavior management, 12, 991-1002. doi:10.2147/PRBM.S179138

Aazh, H., \& Moore, B. C. J. (2018). Effectiveness of Audiologist-Delivered Cognitive Behavioral Therapy for Tinnitus and Hyperacusis Rehabilitation: Outcomes for Patients Treated in Routine Practice. Am J Audiol, 27(4), 547-558. doi:10.1044/2018_aja-17-0096

Andersson, E., Steneby, S., Karlsson, K., Ljótsson, B., Hedman, E., Enander, J., . . . Rück, C. (2014). Long-term efficacy of Internet-based cognitive behavior therapy for obsessive-compulsive disorder with or without booster: a randomized controlled trial. Psychol Med, 44(13), 2877-2887. doi:10.1017/S0033291714000543

Andersson, G. (2003). Tinnitus loudness matchings in relation to annoyance and grading of severity. Auris Nasus Larynx, 30(2), 129-133.

Andersson, G., Carlbring, P., Ljótsson, B., \& Hedman, E. (2013). Guided Internet-Based CBT for Common Mental Disorders. Journal of Contemporary Psychotherapy, 43(4), 223-233. doi:10.1007/s10879-013-9237-9

Andersson, G., Juris, L., Classon, E., Fredrikson, M., \& Furmark, T. (2006). Consequences of suppressing thoughts about tinnitus and the effects of cognitive distraction on brain activity in tinnitus patients. Audiol Neurootol, 11(5), 301-309. doi: $10.1159 / 000094460$

Andersson, G., Kaldo, V., Strömgren, T., \& Ström, L. (2004). Are coping strategies really useful for the tinnitus patient? An investigation conducted via the internet. Audiological Medicine, 2(1), 54-59. doi:doi:10.1080/16513860410027358

Baker, A. L., \& Wilson, P. H. (1985). Cognitive-behavior therapy for depression: The effects of booster sessions on relapse. Behav Ther, 16(4), 335-344. doi:https://doi. org/10.1016/S0005-7894(85)80001-0

Bauer, E. A., MacNamara, A., Sandre, A., Lonsdorf, T. B., Weinberg, A., Morriss, J., \& van Reekum, C. M. (2020). Intolerance of uncertainty and threat generalization: A replication and extension. Psychophysiology, 57(5), e13546. doi:10.1111/psyp.13546

Baumann, M., Stargardt, T., \& Frey, S. (2020). Cost-Utility of Internet-Based Cognitive Behavioral Therapy in Unipolar Depression: A Markov Model Simulation. Applied Health Economics and Health Policy. doi:10.1007/s40258-019-00551-x

Bélanger, C., Blais Morin, B., Brousseau, A., Gagné, N., Tremblay, A., Daigle, K., . . . Léonard, G. (2017). Unpredictable pain timings lead to greater pain when people are highly intolerant of uncertainty. Scand J Pain, 17, 367-372. doi:10.1016/j. sjpain.2017.09.013 
Beukes, E. W., Allen, P. M., Manchaiah, V., Baguley, D. M., \& Andersson, G. (2017). Internet-Based Intervention for Tinnitus: Outcome of a Single-Group Open Trial. $J$ Am Acad Audiol, 28(4), 340-351. doi:10.3766/jaaa.16055

Beukes, E. W., Baguley, D. M., Allen, P. M., Manchaiah, V., \& Andersson, G. (2018). Audiologist-Guided Internet-Based Cognitive Behavior Therapy for Adults With Tinnitus in the United Kingdom: A Randomized Controlled Trial. Ear Hear, 39(3), 423-433. doi:10.1097/aud.0000000000000505

Beukes, E. W., Manchaiah, V., Allen, P. M., Baguley, D. M., \& Andersson, G. (2019). Internet-Based Interventions for Adults With Hearing Loss, Tinnitus, and Vestibular Disorders: A Systematic Review and Meta-Analysis. Trends Hear, 23, 2331216519851749-2331216519851749. doi:10.1177/2331216519851749

Birrell, J., Meares, K., Wilkinson, A., \& Freeston, M. (2011). Toward a definition of intolerance of uncertainty: A review of factor analytical studies of the Intolerance of Uncertainty Scale. Clin Psychol Rev, 31(7), 1198-1208. doi:https://doi.org/10.1016/j. cpr.2011.07.009

Boddez, Y., Baeyens, F., Luyten, L., Vansteenwegen, D., Hermans, D., \& Beckers, T. (2013). Rating data are underrated: Validity of US expectancy in human fear conditioning. Journal of Behavior Therapy and Experimental Psychiatry, 44(2), 201206. doi:https://doi.org/10.1016/j.jbtep.2012.08.003

Cima, R. F. F., Andersson, G., Schmidt, C. J., \& Henry, J. A. (2014). Cognitive-behavioral treatments for tinnitus: a review of the literature. J Am Acad Audiol, 25(1), 29-61. doi:10.3766/jaaa.25.1.4

Cima, R. F. F., Crombez, G., \& Vlaeyen, J. W. (2011). Catastrophizing and fear of tinnitus predict quality of life in patients with chronic tinnitus. Ear Hear, 32(5), 634-641. doi:10.1097/AUD.0b013e31821106dd

Cima, R. F. F., Kikidis, D., Mazurek, B., Haider, H., Cederroth, C. R., Norena, A., . . . Hoare, D. J. (2020). Tinnitus healthcare: a survey revealing extensive variation in opinion and practices across Europe. BMJ Open, 10(1), e029346. doi:10.1136/ bmjopen-2019-029346

Cima, R. F. F., Maes, I. H., Joore, M. A., Scheyen, D. J., El Refaie, A., Baguley, D. M., . . . Vlaeyen, J. W. (2012). Specialised treatment based on cognitive behaviour therapy versus usual care for tinnitus: a randomised controlled trial. Lancet, 379(9830), 19511959. doi:10.1016/s0140-6736(12)60469-3

Cima, R. F. F., van Breukelen, G., \& Vlaeyen, J. W. S. (2017). Tinnitus-related fear: Mediating the effects of a cognitive behavioural specialised tinnitus treatment. Hear Res. doi:10.1016/j.heares.2017.10.003

Cook, T. D., \& Campbell, D. T. (1979). The design and conduct of true experiments and quasiexperiments in field settings. In R. T. Moday \& R. M. Steers (Eds.), Reproduced in part in Research in Organizations: Issues and Controversies Goodyear Publishing Company. 
Crawford, M. J., Robotham, D., Thana, L., Patterson, S., Weaver, T., Barber, R., . . Rose, D. (2011). Selecting outcome measures in mental health: the views of service users. J Ment Health, 20(4), 336-346. doi:10.3109/09638237.2011.577114

Crombez, G., De Paepe, A. L., Veirman, E., Eccleston, C., Verleysen, G., \& Van Ryckeghem, D. M. L. (2020). Let's talk about pain catastrophizing measures: an item content analysis. PeerJ, 8, e8643-e8643. doi:10.7717/peerj.8643

Cuijpers, P., Hollon, S. D., van Straten, A., Bockting, C., Berking, M., \& Andersson, G. (2013). Does cognitive behaviour therapy have an enduring effect that is superior to keeping patients on continuation pharmacotherapy? A meta-analysis. BMJ Open, 3(4), e002542. doi:10.1136/bmjopen-2012-002542

Deale, A., Husain, K., Chalder, T., \& Wessely, S. (2001). Long-Term Outcome of Cognitive Behavior Therapy Versus Relaxation Therapy for Chronic Fatigue Syndrome: A 5-Year Follow-Up Study. American Journal of Psychiatry, 158(12), 2038-2042. doi:10.1176/appi.ajp.158.12.2038

Fischerauer, S. F., Talaei-Khoei, M., Vissers, F. L., Chen, N., \& Vranceanu, A. M. (2018). Pain anxiety differentially mediates the association of pain intensity with function depending on level of intolerance of uncertainty. J Psychiatr Res, 97, 30-37. doi:10.1016/j.jpsychires.2017.11.006

Fuller, T. E., Cima, R. F. F., Langguth, B., Mazurek, B., Vlaeyen, J. W., \& Hoare, D. J. (2020). Cognitive behavioural therapy for tinnitus. Cochrane Database Syst Rev, 1, Cd012614. doi:10.1002/14651858.CD012614.pub2

Fuller, T. E., Cima, R. F. F., Van den Bussche, E., \& Vlaeyen, J. W. S. (2019). The Fear of Tinnitus Questionnaire: Toward a Reliable and Valid Means of Assessing Fear in Adults with Tinnitus. Ear Hear. doi:10.1097/aud.0000000000000728

Fuller, T. E., van Breukelen, G. J. P., Vlaeyen, J. W. S., \& Cima, R. F. F. (2020). Pragmatic uncontrolled study of specialized cognitive behavioural therapy for adults with chronic tinnnitus. (submitted).

Garcia, R. (2017). Neurobiology of fear and specific phobias. Learning \& memory (Cold Spring Harbor, N.Y.), 24(9), 462-471. doi:10.1101/Im.044115.116

Glombiewski, J. A., Holzapfel, S., Riecke, J., Vlaeyen, J. W. S., de Jong, J., Lemmer, G., \& Rief, W. (2018). Exposure and CBT for chronic back pain: An RCT on differential efficacy and optimal length of treatment. J Consult Clin Psychol, 86(6), 533-545. doi: $10.1037 /$ ccp0000298

Glombiewski, J. A., Riecke, J., Holzapfel, S., Rief, W., Konig, S., Lachnit, H., \& Seifart, U. (2015). Do patients with chronic pain show autonomic arousal when confronted with feared movements? An experimental investigation of the fear-avoidance model. Pain, 156(3), 547-554. doi:10.1097/01.j.pain.0000460329.48633.ce

Gloster, A. T., Hauke, C., Höfler, M., Einsle, F., Fydrich, T., Hamm, A., . . Wittchen, H.-U. (2013). Long-term stability of cognitive behavioral therapy effects for panic disorder with agoraphobia: A two-year follow-up study. Behaviour Research and Therapy, 51(12), 830-839. doi:https://doi.org/10.1016/j.brat.2013.09.009 
Grossman, P., Tiefenthaler-Gilmer, U., Raysz, A., \& Kesper, U. (2007). Mindfulness training as an intervention for fibromyalgia: evidence of postintervention and 3-year follow-up benefits in well-being. Psychother Psychosom, 76(4), 226-233. doi:10.1159/000101501

Hall, D. A., Haider, H., Kikidis, D., Mielczarek, M., Mazurek, B., Szczepek, A. J., \& Cederroth, C. R. (2015). Toward a Global Consensus on Outcome Measures for Clinical Trials in Tinnitus: Report From the First International Meeting of the COMiT Initiative, November 14, 2014, Amsterdam, The Netherlands. Trends Hear, 19. doi:10.1177/2331216515580272

Hall, D. A., Haider, H., Szczepek, A. J., Lau, P., Rabau, S., Jones-Diette, J., . . Mazurek, B. (2016). Systematic review of outcome domains and instruments used in clinical trials of tinnitus treatments in adults. Trials, 17, 270. doi:10.1186/s13063-016-1399-9

Hall, D. A., Smith, H., Hibbert, A., Colley, V., Haider, H. F., Horobin, A., . . Core Outcome Measures in Tinnitus, i. (2018). The COMiT'ID Study: Developing Core Outcome Domains Sets for Clinical Trials of Sound-, Psychology-, and PharmacologyBased Interventions for Chronic Subjective Tinnitus in Adults. Trends Hear, 22, 2331216518814384-2331216518814384. doi:10.1177/2331216518814384

Handscomb, L. (2018). A systematic evaluation of the cognitive behavioural model of tinnitus distress. (PhD), University of Nottingham, Nottingham, U.K.

Handscomb, L., Shorter, G. W., Hoare, D. J., \& Hall, D. A. (2019). Evaluation of a Cognitive Behavioral Model of Tinnitus Distress: A Cross-Sectional Study Using Structural Equation Modeling. Ear Hear. doi:10.1097/aud.0000000000000826

Hedman, E., Furmark, T., Carlbring, P., Ljótsson, B., Rück, C., Lindefors, N., \& Andersson, G. (2011). A 5-Year Follow-up of Internet-Based Cognitive Behavior Therapy for Social Anxiety Disorder. J Med Internet Res, 13(2), e39. doi:10.2196/jmir.1776

Heneghan, C., Mahtani, K. R., Goldacre, B., Godlee, F., Macdonald, H., \& Jarvies, D. (2017). Evidence based medicine manifesto for better healthcare. BMJ, 357, j2973. doi:10.1136/bmj.j2973

Henry, J. A., Thielman, E. J., Zaugg, T. L., Kaelin, C., Schmidt, C. J., Griest, S., . . Carlson, K. (2017). Randomized Controlled Trial in Clinical Settings to Evaluate Effectiveness of Coping Skills Education Used With Progressive Tinnitus Management. J Speech Lang Hear Res, 60(5), 1378-1397. doi:10.1044/2016_jslhr-h-16-0126

Henry, J. L., \& Wilson, P. (1999). Cognitive-behavioural therapy for tinnitus-related distress: An experimental evaluation of initial treatment and relapse prevention. Paper presented at the Sixth International Tinnitus Seminar.

Hesser, H., Weise, C., Westin, V. Z., \& Andersson, G. (2011). A systematic review and meta-analysis of randomized controlled trials of cognitive-behavioral therapy for tinnitus distress. Clin Psychol Rev, 31(4), 545-553. doi:10.1016/j.cpr.2010.12.006

Hiller, W., \& Goebel, G. (2006). Factors influencing tinnitus loudness and annoyance. Arch Otolaryngol Head Neck Surg, 132(12), 1323-1330. doi:10.1001/archotol.132.12.1323 
Hinton, D. E., Rivera, E. I., Hofmann, S. G., Barlow, D. H., \& Otto, M. W. (2012). Adapting CBT for traumatized refugees and ethnic minority patients: examples from culturally adapted CBT (CA-CBT). Transcult Psychiatry, 49(2), 340-365. doi:10.1177/1363461512441595

Hoare, D. J., Gander, P. E., Collins, L., Smith, S., \& Hall, D. A. (2012). Management of tinnitus in English NHS audiology departments: an evaluation of current practice. Journal of Evaluation in Clinical Practice, 18(2), 326-334. doi:10.1111/j.13652753.2010.01566.x

Hoare, D. J., Kowalkowski, V. L., Kang, S., \& Hall, D. A. (2011). Systematic review and meta-analyses of randomized controlled trials examining tinnitus management. The Laryngoscope, 121(7), 1555-1564. doi:10.1002/lary.21825

Hodgson, R., \& Rachman, S. (1974). II. Desynchrony in measures of fear. Behav Res Ther, 12(4), 319-326. doi:10.1016/0005-7967(74)90006-0

Hofmann, S. G., \& Asmundson, G. J. (2008). Acceptance and mindfulness-based therapy: new wave or old hat? Clin Psychol Rev, 28(1), 1-16. doi:10.1016/j.cpr.2007.09.003

Hofmann, S. G., Asnaani, A., Vonk, I. J. J., Sawyer, A. T., \& Fang, A. (2012). The Efficacy of Cognitive Behavioral Therapy: A Review of Meta-analyses. Cognitive Therapy and Research, 36(5), 427-440. doi:10.1007/s10608-012-9476-1

Jasper, K., Weise, C., Conrad, I., Andersson, G., Hiller, W., \& Kleinstäuber, M. (2014). Internet-based guided self-help versus group cognitive behavioral therapy for chronic tinnitus: a randomized controlled trial. Psychother Psychosom, 83(4), 234246. doi:10.1159/000360705

Kaldo-Sandstrom, V., Larsen, H. C., \& Andersson, G. (2004). Internet-based cognitivebehavioral self-help treatment of tinnitus: clinical effectiveness and predictors of outcome. Am J Audiol, 13(2), 185-192.

Kaldo, V., \& Andersson, G. (2004). Kognitiv beteendeterapivid tinnitus [CognitiveBehavioural Therapy of Tinnitus]. Lund, Sweden: Studentlitteratur.

Kaldo, V., Haak, T., Buhrman, M., Alfonsson, S., Larsen, H. C., \& Andersson, G. (2013). Internet-based cognitive behaviour therapy for tinnitus patients delivered in a regular clinical setting: outcome and analysis of treatment dropout. Cogn Behav Ther, 42(2), 146-158. doi:10.1080/16506073.2013.769622

Karran, E. L., McAuley, J. H., Traeger, A. C., Hillier, S. L., Grabherr, L., Russek, L. N., \& Moseley, G. L. (2017). Can screening instruments accurately determine poor outcome risk in adults with recent onset low back pain? A systematic review and meta-analysis. BMC Med, 15(1), 13. doi:10.1186/s12916-016-0774-4

Kenter, R. M. F., van de Ven, P. M., Cuijpers, P., Koole, G., Niamat, S., Gerrits, R. S., . . . van Straten, A. (2015). Costs and effects of Internet cognitive behavioral treatment blended with face-to-face treatment: Results from a naturalistic study. Internet Interventions, 2(1), 77-83. doi:https://doi.org/10.1016/j.invent.2015.01.001 
Kirby, S. E., \& Yardley, L. (2009). Cognitions associated with anxiety in Ménière's disease. J Psychosom Res, 66(2), 111-118. doi:https://doi.org/10.1016/j.jpsychores.2008.05.027

Kleinstäuber, M., Jasper, K., Schweda, I., Hiller, W., Andersson, G., \& Weise, C. (2013). The role of fear-avoidance cognitions and behaviors in patients with chronic tinnitus. Cogn Behav Ther, 42(2), 84-99. doi:10.1080/16506073.2012.717301

Kodal, A., Fjermestad, K., Bjelland, I., Gjestad, R., Öst, L.-G., Bjaastad, J. F., . . . Wergeland, G. J. (2018). Long-term effectiveness of cognitive behavioral therapy for youth with anxiety disorders. J Anxiety Disord, 53, 58-67. doi:https://doi. org/10.1016/j.janxdis.2017.11.003

Kraepelien, M., Mattsson, S., Hedman-Lagerlöf, E., Petersson, I. F., Forsell, Y., Lindefors, N., \& Kaldo, V. (2018). Cost-effectiveness of internet-based cognitive-behavioural therapy and physical exercise for depression. BJPsych open, 4(4), 265-273. doi:10.1192/bjo.2018.38

Lang, P. J., Levin, D. N., Miller, G. A., \& Kozak, M. J. (1983). Fear behavior, fear imagery, and the psychophysiology of emotion: the problem of affective response integration. J Abnorm Psychol, 92(3), 276-306. doi:10.1037//0021-843x.92.3.276

Lee, D. Y., Lee, J. Y., \& Kim, Y. H. (2018). Management of tinnitus in children: Review of literature and effect of counseling. Auris Nasus Larynx, 45(4), 667-672. doi:https:// doi.org/10.1016/j.anl.2017.09.002

Leeuw, M., Goossens, M. E., de Vet, H. C., \& Vlaeyen, J. W. S. (2009). The fidelity of treatment delivery can be assessed in treatment outcome studies: a successful illustration from behavioral medicine. J Clin Epidemiol, 62(1), 81-90. doi:10.1016/j. jclinepi.2008.03.008

Lester, K., Resick, P. A., Young-Xu, Y., \& Artz, C. (2010). Impact of race on early treatment termination and outcomes in posttraumatic stress disorder treatment. $J$ Consult Clin Psychol, 78(4), 480-489. doi:10.1037/a0019551

Lonsdorf, T. B., Menz, M. M., Andreatta, M., Fullana, M. A., Golkar, A., Haaker, J., . . . Merz, C. J. (2017). Don't fear 'fear conditioning': Methodological considerations for the design and analysis of studies on human fear acquisition, extinction, and return of fear. Neurosci Biobehav Rev, 77, 247-285. doi:10.1016/j.neubiorev.2017.02.026

Maes, I. H., Cima, R. F. F., Anteunis, L. J., Scheijen, D. J., Baguley, D. M., El Refaie, A., . . J Joore, M. A. (2014). Cost-effectiveness of specialized treatment based on cognitive behavioral therapy versus usual care for tinnitus. Otol Neurotol, 35(5), 787-795. doi:10.1097/mao.0000000000000331

Main, C. J., Kendall, N. A. S., \& Hasenbring, M. I. (Eds.). (2012). Screening of Psychosocial Risk Factors (Yellow Flags) for Chronic Back Pain and Disability. Oxford: OUP Oxford.

Martin-Soelch, C., Stöcklin, M., Dammann, G., Opwis, K., \& Seifritz, E. (2006). Anxiety trait modulates psychophysiological reactions, but not habituation processes related to affective auditory stimuli. International Journal of Psychophysiology, 61(2), 87-97. doi:https://doi.org/10.1016/j.ijpsycho.2005.07.009 
McCormack, A., Edmondson-Jones, M., Somerset, S., \& Hall, D. (2016). A systematic review of the reporting of tinnitus prevalence and severity. Hear Res, 337, 70-79. doi:10.1016/j.heares.2016.05.009

McKenna, L., Handscomb, L., Hoare, D. J., \& Hall, D. A. (2014). A scientific cognitivebehavioral model of tinnitus: novel conceptualizations of tinnitus distress. Front Neurol, 5, 196. doi:10.3389/fneur.2014.00196

McKenna, L., Marks, E. M., Hallsworth, C. A., \& Schaette, R. (2017). Mindfulness-Based Cognitive Therapy as a Treatment for Chronic Tinnitus: A Randomized Controlled Trial. Psychother Psychosom, 86(6), 351-361.

McLaughlin, K. A., \& Nolen-Hoeksema, S. (2011). Rumination as a transdiagnostic factor in depression and anxiety. Behav Res Ther, 49(3), 186-193. doi:10.1016/j.brat.2010.12.006

McLellan, L. F., Peters, L., \& Rapee, R. M. (2016). Measuring Suitability for Cognitive Behavior Therapy: A Self-Report Measure. Cognitive Therapy and Research, 40(5), 687-704. doi:10.1007/s10608-016-9771-3

McLellan, L. F., Stapinski, L. A., \& Peters, L. (2019). Pre-treatment CBT-Mindedness Predicts CBT Outcome. Cognitive Therapy and Research, 43(2), 303-311. doi:10.1007/ s10608-018-9977-7

Mehta, S., Peynenburg, V. A., \& Hadjistavropoulos, H. D. (2019). Internet-delivered cognitive behaviour therapy for chronic health conditions: a systematic review and meta-analysis. J Behav Med, 42(2), 169-187. doi:10.1007/s10865-018-9984-x

Mokkink, L. B., Prinsen, C. A., Patrick, D. L., Alonso, J., Bouter, L. M., Vet, H. C. d., \& Terwee, C. B. (2019). COSMIN Study Design checklist for Patient-reported outcome measurement instruments. Retrieved from https://www.cosmin.nl/tools/checklistsassessing-methodological-study-qualities/:

Montgomery, A. A., Peters, T. J., \& Little, P. (2003). Design, analysis and presentation of factorial randomised controlled trials. BMC Med Res Methodol, 3, 26-26. doi:10.1186/1471-2288-3-26

Myhr, G., Talbot, J., Annable, L., \& Pinard, G. (2007). Suitability for Short-Term Cognitive-Behavioral Therapy. Journal of Cognitive Psychotherapy, 21, 334-345. doi:10.1891/088983907782638743

Phillips, J. S., McFerran, D. J., Hall, D. A., \& Hoare, D. J. (2018). The natural history of subjective tinnitus in adults: A systematic review and meta-analysis of no-intervention periods in controlled trials. Laryngoscope, 128(1), 217-227. doi:10.1002/lary.26607

Quartana, P. J., Campbell, C. M., \& Edwards, R. R. (2009). Pain catastrophizing: a critical review. Expert review of neurotherapeutics, 9(5), 745-758. doi:10.1586/ERN.09.34

Rademaker, M. M., Stegeman, I., Ho-Kang-You, K. E., Stokroos, R. J., \& Smit, A. L. (2019). The Effect of Mindfulness-Based Interventions on Tinnitus Distress. A Systematic Review. Front Neurol, 10, 1135. doi:10.3389/fneur.2019.01135 
Renaud, J., Russell, J. J., \& Myhr, G. (2014). Predicting Who Benefits Most From Cognitive-Behavioral Therapy for Anxiety and Depression. J Clin Psychol, 70(10), 924-932. doi:10.1002/jclp.22099

Robinson, S. K., Viirre, E. S., Bailey, K. A., Kindermann, S., Minassian, A. L., Goldin, P. R., . . McQuaid, J. R. (2008). A randomized controlled trial of cognitive-behavior therapy for tinnitus. Int Tinnitus J, 14(2), 119-126.

Rosing, S. N., Schmidt, J. H., Wedderkopp, N., \& Baguley, D. M. (2016). Prevalence of tinnitus and hyperacusis in children and adolescents: a systematic review. BMJ Open, 6(6), e010596-e010596. doi:10.1136/bmjopen-2015-010596

Rosser, B. A. (2019). Intolerance of Uncertainty as a Transdiagnostic Mechanism of Psychological Difficulties: A Systematic Review of Evidence Pertaining to Causality and Temporal Precedence. Cognitive Therapy and Research, 43(2), 438-463. doi:10.1007/s10608-018-9964-z

Sackett, D. L., Rosenberg, W. M. C., Gray, J. A. M., Haynes, R. B., \& Richardson, W. S. (1996). Evidence based medicine: what it is and what it isn't. BMJ, 312(7023), 71-72. doi:10.1136/bmj.312.7023.71

Sales, C. M. D., \& Alves, P. C. G. (2016). Patient-Centered Assessment in Psychotherapy: A Review of Individualized Tools. Clinical Psychology: Science and Practice, 23(3), 265-283. doi:10.1111/cpsp.12162

Sedgwick, P. (2012). Randomised controlled trials with full factorial designs. BMJ : British Medical Journal, 345, e5114. doi:10.1136/bmj.e5114

Shapiro, M. B. (1961). A method of measuring psychological changes specific to the individual psychiatric patient*. British Journal of Medical Psychology, 34(2), 151-155. doi:10.1111/j.2044-8341.1961.tb00940.x

Smith, H., Fackrell, K., Kennedy, V., Barry, J., Partridge, L., \& Hoare, D. J. (2019). A scoping review to catalogue tinnitus problems in children. International Journal of Pediatric Otorhinolaryngology, 122, 141-151. doi:https://doi.org/10.1016/j. ijporl.2019.04.006

Tate, R. L., Perdices, M., Rosenkoetter, U., Shadish, W., Vohra, S., Barlow, D. H., . . Wilson, B. (2016). The Single-Case Reporting Guideline In BEhavioural Interventions (SCRIBE) 2016 Statement. Neuropsychol Rehabil, 1-15. doi:10.1080/09602011.2016.1190533

Vlaeyen, J. W. S., \& Morley, S. (2005). Cognitive-behavioral treatments for chronic pain: what works for whom? Clin J Pain, 21(1), 1-8. doi:10.1097/00002508-200501000-00001

Vlaeyen, J. W. S., Wicksell, R. K., Simons, L. E., Gentili, C., De, T. K., Tate, R. L., . . Onghena, P. (2020). From Boulder to Stockholm in 70 Years: Single Case Experimental Designs in Clinical Research. The Psychological record. doi:10.1007/ s40732-020-00402-5 
von Brachel, R., Hirschfeld, G., Berner, A., Willutzki, U., Teismann, T., Cwik, J. C., . . . Margraf, J. (2019). Long-Term Effectiveness of Cognitive Behavioral Therapy in Routine Outpatient Care: A 5- to 20-Year Follow-Up Study. Psychother Psychosom, 88(4), 225-235. doi:10.1159/000500188

Waltman, S. H., Sokol, L., \& Beck, A. T. (2017). Cognitive Behavior Therapy Treatment Fidelity in Clinical Trials: Review of Recommendations. Current Psychiatry Reviews, 13(4), 311-315. doi:http://dx.doi.org/10.2174/1573400514666180109150208

Wesner, A. C., Gomes, J. B., Detzel, T., Guimarães, L. S. P., \& Heldt, E. (2015). Booster Sessions after Cognitive-Behavioural Group Therapy for Panic Disorder: Impact on Resilience, Coping, and Quality Of Life. Behavioural and Cognitive Psychotherapy, 43(5), 513-525. doi:10.1017/S1352465814000289

Wiltsey Stirman, S., Marques, L., Creed, T. A., Gutner, C. A., DeRubeis, R., Barnett, P. G., . . . La Bash, H. (2018). Leveraging routine clinical materials and mobile technology to assess CBT fidelity: the Innovative Methods to Assess Psychotherapy Practices (imAPP) study. Implementation Science, 13(1), 69. doi:10.1186/s13012-018-0756-3 


\section{Valorisation addendum}

Valorisation, in addition to research and teaching, has become an important function of universities in the Netherlands. It can be defined as the process of using academic knowledge to "add value" to society through impact on/in, for example, policy, commerce, and health.

Fundamentally, society can benefit most widely from research if it is freely accessible (Wang, Liu, Mao, \& Fang, 2015). It follows that if research articles do not fully present the details of how a study was conducted and what the results were in a transparent and complete way, it makes it very difficult for it to be "consumed" by others (i.e., patients, clinicians, researchers and policy-makers to name a few). Researchers, patients or policy makers cannot act on or use the information if they do not know about it and/or cannot access it. Thus in essence making our research as accessible as possible has been one way we have attempted to valorise our findings. In order to make the results as accessible as possible, we have chosen to publish open access and/or make pre-prints available through Researchgate or data repositories; and, provided extensive detailed results and make other materials available as supplementary digital content to accompany publications. In addition to this, we have also registered and/or published study protocols (e.g. Chapters 2 and 3). By doing so, our methods have been peer-reviewed ahead of conducting the studies and thus (hopefully) improving the quality and integrity of results. Lastly, through publishing the protocols and registering the studies, we hope to have minimised the chances of unintended duplication of studies by other research groups, as well as incorporating a safeguard against incomplete or biased reporting of results.

Following, we describe how society can benefit from the results of this dissertation. Specifically, we propose how: people with tinnitus; policy makers; researchers; and, clinicians working with people with tinnitus might use and hopefully benefit from our findings.

\section{We have generated high quality information about the safety, efficacy and effectiveness of CBT for tinnitus}

Using the Internet to obtain information about health conditions is convenient and a common practice. Approximately one in 20 of all searches on Google is for health related information (Ennis-O'Connor, 2018). However, when it comes to information available on the Internet about tinnitus, research has consistently found that what exists is highly variable in terms of quality and readability (Kieran, Skinner, Donnelly, \& Smyth, 2010; Manchaiah et al., 2019; McKearney, MacKinnon, Smith, \& Baker, 2018). Similarly, there is a large number of smartphone applications, also of varying quality, that people with tinnitus use to supplement/complement the information about tinnitus they receive from healthcare providers (Deshpande \& Shimunova, 2019). Poor quality information about tinnitus might be misleading, inaccurate, or reflect the commercial interests of the provider of the information. For example, a recent cross-sectional study examining tinnitus related videos on You Tube found that although they were mostly about the personal experience of living with tinnitus, people with tinnitus (who were the majority of people posting videos) were equally likely as companies or clinicians to be selling or 
promoting a particular product or service (Basch et al., 2018). The personal or financial interests of the presenter in the video thus might lead tinnitus patients to overestimate the benefits and underestimate the risks of a particular product.

In contrast to this, the methods we used to conduct the systematic review and metaanalysis of CBT generated a (relatively) unbiased account of its efficacy. We conducted the systematic review and meta-analysis (Chapter 3 ) in collaboration with and according to the strict methodological standards of the Cochrane Organisation. [Cochrane is an international collaboration of researchers, healthcare providers, patients and carers whose mission is to generate high quality, accessible evidence that addresses questions about efficacy and safety of healthcare interventions.] Cochrane does not accept any commercial or "conflicted" funding and employs methods and standards that minimises the influence of biases of researchers conducting systematic reviews and meta-analysis. Because of the enforcement of the highest methodological standards required by Cochrane, Cochrane reviews are considered the benchmark for information about healthcare. Thus, with the production of our systematic review and meta-analysis of CBT for tinnitus, we have generated information, also presented in "plain language", that can be used by patients, policy-makers and healthcare professionals when considering what treatment options to use, fund or deliver for tinnitus related distress and interference. Indeed, our review has recently been referred to in a parliamentary inquiry into research and service provision for tinnitus sufferers conducted by House of Commons, United Kingdom. Even if, for example, policy makers or patients are prevented by financial constraints from implementing or using CBT, the results demonstrating its safety and efficacy can influence debate around and the demand for the provision of CBT for tinnitus. In other words, by generating evidence that CBT is safe and effective (at least in the shortterm), we can shape/influence debate about service provision (Elliott \& Popay, 2000).

In addition to patients and policymakers, other tinnitus researchers can use the results of the review to guide future studies they conduct. For example, the review can inform their choice of outcome measure, type of treatment/CBT component, comparator intervention and follow-up duration. In particular, establishing the longer-term effectiveness of CBT for tinnitus should be considered a research priority.

\section{Improving access to treatment for tinnitus patients in the Netherlands}

In the course of conducting the systematic review and meta-analysis of randomised controlled trials of CBT for tinnitus it became clear that pragmatic trials of CBT were rare. Given that, policy-makers and clinicians experience uncertainty about how the interventions would actually work in practice. Chapter 4 addresses this issue by demonstrating that it is indeed possible to provide specialised stepped-care CBT for tinnitus (Cima et al., 2012) in settings and under conditions more readily found in everyday care in the Netherlands.

The results have already had an impact on the treatment of tinnitus in the Netherlands. Specifically, and in conjunction with Chapter 3, the preliminary results contributed to the evidence base underpinning the Dutch clinical guidelines for the assessment and treatment of tinnitus that were published in December 2016 (Dutch Association for Ear 
Nose Throat and Head surgery [Nederlandse Vereniging voor Keel - Neus - Oor heel kunde en Heelkunde van het Hoofd -Halsgebied], 2016). Furthermore, from 2019 specialised CBT for tinnitus was included in the standard health insurance package, thereby allowing greater numbers of people to access treatment, albeit with the caveat that currently there are only a limited number of services accredited to deliver the intervention. The current challenge is thus to build workforce capacity to deliver the intervention.

The results from the study can also be used as a reference point for future evaluations of treatment effectiveness in the Netherlands.

\section{Helping inform assessment and treatment of tinnitus across Europe and beyond ...gathering comprehensive data is a time-consuming process. When time is limited, it can be far too convenient to form policy recommendations based on common sense or intuition, authority, habit, status quo, or tradition. (p. 5; Umphrey \& Miraglia, 2017)}

This quote further highlights the importance of making research findings as accessible and consumable as possible in order to be used. If policy makers cannot interpret the results easily or if consumers/patients do not understand whether one intervention is better than another, decisions they make about preferences, cannot be considered to be informed.

The pathways to access assessments and interventions for tinnitus have long been recognised as fragmented and inconsistent. Fragmented and variable assessment and treatment strategies for tinnitus can produce inequalities between patients within the healthcare system as well as exacerbating distress and disability as people either cannot access help or can only do so after delays. As part of the Tinnet initiative (https://tinnet. tinnitusresearch.net/), we undertook the systematic review of clinical guidelines (Chapter 2) to inform the development of the European clinical guideline for assessment and treatment of tinnitus. The review confirmed the assumption held by clinicians and researchers that there was a lack of existing clinical guidelines, but also highlighted that there were more similarities than differences between the recommendations of particular assessment and treatment strategies. Not only did the review contribute to the development of the European clinical guideline, but it has been viewed nearly 30,000 times, has an Altmetric score of 55 (in the top $5 \%$ of research articles), and already been cited 20 times. While the value of such metrics might be debatable, they provide a crude indication of its interest and relevance to a wide audience.

\section{An assessment tool for psychologists, audiologists, ENTs and other clini- cians working with people with tinnitus}

Tinnitus related fear, as measured by the Fear of Tinnitus Questionnaire has been shown to be associated with quality of life (Cima, Crombez, \& Vlaeyen, 2011) and mediate improvements in patients level of distress (Cima, van Breukelen, \& Vlaeyen, 2017). However, the actual psychometric properties of the questionnaire had, until now, not been examined. A lack of data about a questionnaire conceivably affects the level of confidence one has in results and thus potentially limits/reduces its uptake. With the publication of Chapter 5 , we have shown that tinnitus researchers can use the FTQ as an outcome measures and clinical psychologists can use a three-factor version to inform discussions within therapy. 


\section{References}

Basch, C. H., Yin, J., Kollia, B., Adedokun, A., Trusty, S., Yeboah, F., \& Fung, I. C.-H. (2018). Public online information about tinnitus: A cross-sectional study of YouTube videos. Noise Health, 20(92), 1-8. doi:10.4103/nah.NAH_32_17

Cima, R. F. F., Crombez, G., \& Vlaeyen, J. W. (2011). Catastrophizing and fear of tinnitus predict quality of life in patients with chronic tinnitus. Ear Hear, 32(5), 634641. doi:10.1097/AUD.0b013e31821106dd

Cima, R. F. F., Maes, I. H., Joore, M. A., Scheyen, D. J., El Refaie, A., Baguley, D. M., . . . Vlaeyen, J. W. (2012). Specialised treatment based on cognitive behaviour therapy versus usual care for tinnitus: a randomised controlled trial. Lancet, 379(9830), 19511959. doi:10.1016/s0140-6736(12)60469-3

Cima, R. F. F., van Breukelen, G., \& Vlaeyen, J. W. S. (2017). Tinnitus-related fear: Mediating the effects of a cognitive behavioural specialised tinnitus treatment. Hear Res. doi:10.1016/j.heares.2017.10.003

Deshpande, A. K., \& Shimunova, T. (2019). A Comprehensive Evaluation of Tinnitus Apps. Am J Audiol, 1-12. doi:10.1044/2019_aja-18-0135

Dutch Association for Ear Nose Throat and Head surgery [Nederlandse Vereniging voor Keel - Neus - Oor heel kunde en Heelkunde van het Hoofd -Halsgebied]. (2016). Guideline Tinnitus [Richtlijn Tinnitus]. Retrieved from Utrecht, Netherlands: https:// richtlijnendatabase.nl/richtlijn/tinnitus/tinnitus___startpagina.html

Elliott, H., \& Popay, J. (2000). How are policy makers using evidence? Models of research utilisation and local NHS policy making. Journal of Epidemiology and Community Health, 54(6), 461. doi:10.1136/jech.54.6.461

Ennis-O'Connor, M. (2018). As Google Turns 20, What's Next For Health Information Searching Online? Retrieved from https://hcsmmonitor.com/2018/09/27/as-drgoogle-turns-20-whats-next-for-health-information-searching-online/

Kieran, S. M., Skinner, L. J., Donnelly, M., \& Smyth, D. A. (2010). A critical evaluation of Web sites offering patient information on tinnitus. Ear Nose Throat J, 89(1), E11-14.

Manchaiah, V., Dockens, A. L., Flagge, A., Bellon-Harn, M., Azios, J. H., Kelly-Campbell, R. J., \& Andersson, G. (2019). Quality and Readability of English-Language Internet Information for Tinnitus. J Am Acad Audiol, 30(1), 31-40. doi:10.3766/jaaa.17070

McKearney, R. M., MacKinnon, R. C., Smith, M., \& Baker, R. (2018). Tinnitus information online - does it ring true? J Laryngol Otol, 132(11), 984-989. doi:10.1017/s0022215118001792

Umphrey, L. R., \& Miraglia, P. R. (2017). Evidence-Based Policy Making. In M. Allen (Ed.), The SAGE Encyclopedia of Communication Research Methods. Thousand Oaks, California: SAGE.

Wang, X., Liu, C., Mao, W., \& Fang, Z. (2015). The open access advantage considering citation, article usage and social media attention. Scientometrics, 103(2), 555-564. doi:10.1007/s11192-015-1547-0 


\section{Acknowledgments}

It seems strange in a way that a $\mathrm{PhD}$ is awarded to a single person since everyone knows that many people are involved and make significant contributions to it. I certainly could not have completed it without the assistance and involvement of many wonderful people and would not have enjoyed it anywhere near as much as I did without the involvement of many more. Here, I would like to thank the following people for the special contributions they made.

Professor Johan WS Vlaeyen, or more simply, Johan. First and foremost, thank you very much for the opportunity to work with you. While tinnitus might not be your main line of research, I have always appreciated the insightful and challenging questions you raised during our discussions on conceptual or methodological matters related to studying tinnitus. Despite being committed to so many projects, your ability to meet my deadlines and provide feedback on my work has continually astounded me. I don't know how you do it. Your influential body of work on pain, your thoroughness, rigour and your ability (or at least aspiration) to live a balanced life will inspire me long into the future. Thank you.

Rilana, thank you too so much for the opportunity learn about and make a contribution to the field of tinnitus research. Considering that I did not even realise that I had tinnitus when I first applied for the position on the project, let alone that there were a bunch of people interested in studying it, I appreciated the confidence that you had in my abilities as a researcher to undertake the $\mathrm{PhD}$. From the beginning, you trusted and gave me as much independence and guidance as I needed. Your generosity with introductions to colleagues, and inclusion in the Tinnet projects was pivotal to the completion of major pieces of work included here. In those early days in particular, you enabled me to build working relationships with colleagues in the field who would subsequently go on to become co-authors with us. Thank you.

Derek, I almost feel that you have been another supervisor during this $\mathrm{PhD}$. While we have "only" worked closely on the systematic review, your expertise, experience, and critical yet supportive guidance has been precious. I like to think that when we spoke the first time during a poster session in Nottingham that it was your friendly and open disposition that was the beginning of our collaboration. Four or more years later, the review would not have become what it is without you and nor would it have been completed when it was... in fact I'm sure we'd still be going if it wasn't for your contribution. I am eternally grateful for your generous support.

Professors Gerard van Breukelen and Eva Van den Bussche thank you both so much for your statistical advice and for guidance. Your respective contributions to two of the studies made the papers stronger and better than they would have been without your involvement. You both had a way of delivering advice and instruction akin to a stern but caring mentor that enabled me to keep persevering. Gerard, I also have to say that I enjoyed your passion for the practice of doing better, open and replicable statistics. I hope 
that there are researchers out there interested enough in what we have done to benefit from the extra effort in documenting the statistical methods and rationale we followed.

Professors Love, Tony and Kristina, you probably don't realise it, but you have been like my long distance academic parents. While we don't get to see each other often, I have always appreciated your gentle, curious yet probing questions whenever and wherever we have met up. You have both served as humble role models and exemplified the merits of thoughtful, considered research, that benefits others above your interests. And Tony, my fellow LFC tragic, how I loved receiving your thoughts on the sporadic highs and frequent lows that is part of following our team. Knowing that someone out there is suffering in the same kind of way makes it easier and all the more enjoyable even if we're not in the same hemisphere.

To the Examination Committee and Members of the Opposition, thank you so much for making time in your busy schedules to read my dissertation and undoubtedly, ask me some challenging and thought provoking questions. Thank you! I look forward to meeting you and discussing the dissertation as well as other highs and challenges of conducting research in these times.

My fellow PhDs - Anke, Astrid, Bart, Eveliina, Iris, Jana, Kai, Kristoph, Leo, Martijn, Matheus, Mathilde, Ola, Rena and Sieske - you have simply made the experience of being a PhD candidate better. Whether it has been EPP fun trains, the Banditos express, playing (board/bored)games, having drinks anywhere, or having conversations about life, films, literature, research or supervisors etc etc, you have made it all worthwhile. Thank you! A special shout to Matheus for always helping me realise that it could be worse.

Dear EHP colleagues - Madelon, Johan, Linda, Marjolein, Marieke, Anne, Hanne, Sjaan, Pauline, Kai, Matheus, Mathilde, Kristof, Ola, Rena and Jantine - over the years you have all helped make the experience more than just a training for a career in research. You have been the family within the Faculty that has helped build a closer connection and identity with our discipline. Like the best of families, you have been supportive but challenging; my research ideas, presentations and methods have all been improved because of you.

To Nicole, Lotte and Jill, your wisdom and humour have been priceless in keeping life and work in perspective. Through the difficult times, you have been a great help with solutions and suggestions on how to keep going.

Jessie, Marionne, Lindy and Caroline - how would PhD life function without you? You have been amazing, supportive, curious and patient with me throughout my time in the Faculty. Our conversations about holidays, family life, coffee, and happily to a lesser degree (as no one really wants to talk about work on a coffee-break) research projects, have helped keep me going. In so many ways, you're what helps keep the institution and PhD's going... and of course, you have all been responsible at various times for the conscientious provision of coffee coins. Thus the caffeine that has fuelled so many PhD's before me, can now claim another credit. Thank you, for really making it possible. 
To Dyon, Rachelle, Ken, Saskia, and Pauline at Adelante I am grateful for the all that you shared with me at Adelante as I struggled with Dutch and to get a sense of what CBT for tinnitus actually looked like when delivered to patients.

To the team at Libra, I am grateful for the opportunity to have been able to work with you all. A special thanks though to Petra, Guido, Joep, and Marjo for helping make the trial happen. Through the numerous challenges that arose with the data collection and running the interventions your trust in me and perseverance helped make it possible. Thank you.

To my climbing buddies, Iris, Fania, Gwyn, Lauren, and the institutions of Ivy and Radium - thank you for being you. I only wish that my broken ankle and parenthood hadn't made me such an unreliable partner...

To LFC, thank you so much for ending the wait in this year, 2020, of all years. Wow; what a team! YNWA

A big thank you to Nicole and Sibren for your friendship over the years in Maastricht. Not only did you help Anna I settle into Maastricht right from the beginning, but also you have helped make our time here so enjoyable. Without you Nicole, I literally do not know where we would be (geographically speaking). Your efforts to keep us in Maastricht by suggesting I apply for the PhD were, as we now know, successful.

Jessica and Kai, I cannot remember when we actually met or became friends but it feels like we have known each other so well, for so much longer than we actually have. I have trusted you with anything and everything and found you to be wonderfully generous as confidantes, interrogators, friends and colleagues. Thank you.

Jessica and Nicole, I feel honoured to have you as my paranymphs on the occasion of my defense, and to be able to count you as dear friends for the rest of the time. You are both inspiring. Thank you so much.

To my dear, far away friends Alice, Doug, Tom, Em and Joe... thank you for all your love and support. I treasure it immensely and would be lost without it.

Mum and Dad, your love and support has provided the foundation on which I have been able to do so much. Without you, it would not have been possible. I am eternally grateful for everything.

Andy, Nuning and Cahaya, I have loved sharing times together here in Maastricht, Leiden and in Melbourne. You've made life in the Netherlands feel more like home, provided inspiration, and I am excited to be seeing you here again.

Dear Anna and Bastian, in a typically understated way, I want to thank you so much for all that you've shared, given and enabled me to do during the PhD.... Not least of all, your love, and helping me try to keep working life in check and to take life less seriously. I cannot wait to experience post-PhD life with you. 


\section{CV}

Thomas Edward Fuller was born on $11^{\text {th }}$ May 1976 in Adelaide, Australia. He grew up in Melbourne and went on to graduate from The University of Melbourne with a Bachelor of Arts (majors in Psychology and Criminology) in 1997. He subsequently completed a Postgraduate Diploma of Health Psychology at La Trobe University in 1999 before travelling around and working in Europe for three years. On returning to Melbourne, he enrolled in and went on to complete a Doctorate in Health Psychology at La Trobe University. During the course of this degree, he conducted research on the psychological factors that predict treatment response to CBT for insomnia and completed his clinical psychological training. Following this, he enjoyed working simultaneously in clinical positions in health services and project and policy positions at the Australian Psychological Society respectively.

In 2012 he moved to the England and worked as an Associate Research Fellow at the Medical School of the University of Exeter. Following this position, he settled in Maastricht and resumed clinical work for a short period before beginning his $\mathrm{PhD}$ under the supervision of Prof. Dr. Johan Vlaeyen and Dr Rilana Cima within the Experimental Health Psychology group at the Department of Clinical Psychological Science, Maastricht University. Throughout his career as a psychologist and researcher, Thomas has strived to maintain the highest ethical, clinical and methodological standards and is driven to improve access to and the quality of psychological interventions. He now works as a Senior Clinical Evidence Specialist within the spinal group at Medtronic. 


\section{Publications}

Fuller, T., van Breukelen G. J.P., Vlaeyen, J. W.S., Cima, R.F.F. (under review) Pragmatic uncontrolled study of specialized cognitive behavioural therapy for adults with chronic tinnitus.

Fuller, T., Cima, R., Langguth, B., Mazurek, B., Vlaeyen, J.W.S., Hoare, D.J. (2020) Cognitive behavioural therapy for tinnitus. Cochrane Database of Systematic Reviews. 2020, Jan 8;1(1):CD012614. doi: 10.1002/14651858.CD012614.pub2.

Fuller, T., Vlaeyen, J. W.S., Van den Bussche, E., Cima, R.F.F. (2019) The Fear of Tinnitus Questionnaire (FTQ): Toward a reliable and valid means of assessing fear in adults with tinnitus. Ear and Hearing, published ahead of print.

Genitsaridi, E., Partyka, M., Gallus, S., Lopez-Escamez., Schecklmann, M., Fuller, T. et al. (2019) Standardised profiling for tinnitus research: The European School for Interdisciplinary Tinnitus Research Screening Questionnaire (ESIT-SQ). Hearing Research.

Fuller, T., Haider, H. F., Kikidis, D., Lapira, A., Mazurek, B., Norena, A., . . . Cima, R. F. F. (2017). Different Teams, Same Conclusions? A Systematic Review of Existing Clinical Guidelines for the Assessment and Treatment of Tinnitus in Adults. Frontiers in Psychology, 8(206). doi:10.3389/fpsyg.2017.00206

Fuller, T. (2017) Following \#ehps2017 back to the future. European Health Psychologist, 19(5); 350-1.

Fuller, T., Cima, R., Langguth, B., Mazurek, B., Waddell, A., Hoare, D. J., \& Vlaeyen, J. W. S. (2017). Cognitive behavioural therapy for tinnitus (Protocol). Cochrane Database of Systematic Reviews(4). doi:10.1002/14651858.CD012614

Fuller, T. (2017) Chronic pain (editorial). European Health Psychologist, 19(1); 287-9.

Fuller T. (2016) Tweeting \#EHPSDHP. European Health Psychologist, 18(5); 220-2.

Kassavou, K., Kwasnicka, D., \& Fuller, T. (2016) Mentoring and guidance highlights. European Health Psychologist, 18(4); 164-5.

Hall D., Haider H, Szczepek A., Lau P., Rabau S., Jones-Diette J., Londero A., Edvall N., Cederroth C., Mielczarek M., Fuller T., et al. (2016) Systematic review of outcome domains and instruments used in clinical trials of tinnitus treatments in adults. Trials, 17:270 DOI: 10.1186/s13063-016-1399-9

Fuller, T., Pearson, M., \& Peters, J. (2016) Improving the reporting of health and psychological research, Australian Psychologist, 51(3); 182-7. doi: 10.1111/ap.12135 
Harris, A. \& Fuller, T. (2015) The hollow knock and other sounds in recipes. Gastronomica: The Journal of Critical Food Studies. 15(4);14-17. doi: 10.1525/gfc.2015.15.4.14 (photo credits)

Fuller, T. \#ehps2015 - virtually there. (2015) European Health Psychologist, 17(5); 240-242.

Fuller, T., Pearson, M., Peters, J. \& Anderson, R. (2015) Why don't authors and editors use reporting guidelines? Findings from an online survey and qualitative interviews. PLoS ONE 10(4): e0121585. doi:10.1371/journal.pone.0121585

Fuller, T., \& Kroese, F. (2015) Tailor made for Health Psychology: Issues in the design and effectiveness of Internet interventions. European Health Psychologist, 17(1); 2-5.

Fuller, T., Peters, J., Pearson, M., \& Anderson, R. (2014) 10 years on... The impact of the Transparent Reporting of Evaluations and Non-randomised Design reporting guideline? American Journal of Public Health, 104(11), e110-e117. doi:10.2105/AJPH.2014.302195

Harris, A. \& Fuller, T (2014) The night side of hospitals. Places.http://places. designobserver.com/feature/night-side-of-hospitals-migrant-doctors/38370/

Fuller, T., Pearson, M., \& Peters J. (2013) Transparent reporting - the foundation for full disclosure. European Health Psychologist, 15(3); 67-68.

Fuller, T., Pearson, M., Peters, J. \& Anderson, R. Evaluating the impact and use of the Transparent Reporting of Evaluations and Non-randomised Design (TREND) reporting guidelines: Study protocol. BMJ Open 2012;2:e002073. doi:10.1136/ bmjopen-2012-002073

Fuller, T. (2012) Letter to the editor - Welcome moves towards transferable qualifications. InPsych, 34(5): 20

Harris, A., Delany, C. \& Fuller, T. (2012) Transition in Practice: A Critical Reflection Group for New Doctors - A Facilitator's Manual. Centre for Health and Society, The University of Melbourne, Melbourne; Australia.

Fuller, T., Stokes, D., \& Mathews, R. (2012) Internet supported psychological interventions. Report for the Australian Psychological Society, Melbourne; Australia.

Fuller, T., \& Stokes, D. (2009) Internet-based psychological services and products. InPsych 30(6); 34

Fuller, T., Anderson, M. \& Lindner, H. (2008) Postgraduate placements for psychology students in alcohol and other drug services. InPsych, 30(5); 23.

Fuller, T. \& Greenwood, K. M. (2008) Perceptions of compared with beliefs about sleep: the merits of an illness perception questionnaire for sleep problems. Australian Journal of Psychology, 60(supplement 2008); 139. 
Fuller, T., Davey, C., White, V., \& Peipers, A. (2004) Bowel program information session study. Melbourne, Australia: Report for the Centre for Behavioural Research in Cancer, Cancer Council of Victoria.

Germain, D., Letcher, T., \& Fuller, T. (2004) Evaluation of the Victorian Fresh Start Short Course: January 2000 to February 2003. CBRC Research Paper Series No. 7. Melbourne, Australia: CBRC, The Cancer Council Victoria, June 2004.

\section{Conference presentations}

Fuller, T.E., Cima, R.F.F. \& Vlaeyen, J.W.S. (2018) Deconstructing CBT for tinnitus. VGCT Annual Conference, Veldhoven, The Netherlands

Fuller, T.E., Cima, R.F.F., Langguth, B., Mazurek, B., Waddell, A., Hoare, D.J. \& Vlaeyen, J.W.S. (2018) Is CBT for tinnitus effective? A progress report on a new Cochrane systematic review. Tinnitus Research Initiative Conference, Regensburg, Germany.

Fuller, T.E., Cima, R.F.F. \& Vlaeyen, J.W.S. (2018) CBT for tinnitus: successful replication of a stepped care approach. Tinnitus Research Initiative Conference, Regensburg, Germany.

Fuller, T.E., Cima, R.F.F. \& Vlaeyen, J.W.S. (2018) Efficacy and Effectiveness of CBT for tinnitus. Experimental Psychopathology Postgraduate School. Heeze, The Netherlands.

Fuller T., Cima R., \& Vlaeyen JWS (2017). CBT for tinnitus: successful replication of a stepped care approach. European Association of Behavioural and Cognitive Therapies, Ljubljana, Slovenia.

Fuller T., Cima R., \& Vlaeyen JWS (2016) EMA and Daily diary methods in a single case series study. TINNET Think tank on Ecological Momentary Assessment, Munich, Germany.

Fuller T., Cima R., \& Vlaeyen JWS (2016). The Replicated Single Case Experimental Design as an innovative method for assessing effectiveness of treatment. $10^{\text {th }}$ International Tinnitus Research Initiative Conference, Nottingham, England.

Fuller T., Cima R., \& Vlaeyen JWS (2015) Unpacking CBT treatments for tinnitus. Pain Research Meeting, Genk, Beglium.

Fuller T., Garnefski N., \& Kraaij V. (2014) Non-use of internet interventions for people with a chronic health condition. International Society for Research on Internet Interventions, Valencia, Spain. 
Fuller, T., Pearson, M., Peters, J. \& Anderson, R. (2013) Reporting guidelines - improving the evidence base of public health research. European Public Health Association, Brussels, Belgium.

Fuller, T., Pearson, M., Peters, J. \& Anderson, R. (2012) Examining the uptake of the TREND reporting guidelines. EQUATOR symposium, Freiburg, Germany.

Lindner, H., \& Fuller, T. (2011) Improving interdisciplinary care for people with chronic conditions. 46th Annual Australian Psychological Society Conference, Australia.

Lindner, H., \& Fuller, T. (2010) Chronic Disease Self-Management training: health professional uptake in daily practice. International Conference of Applied Psychology, Australia.

Fuller, T. (2010) The digital clinic - opportunities and responsibilities for psychologists. International Conference of Applied Psychology, Australia.

Harris, A. \& Fuller, T. (2010) Expand your horizons: a lecture series by international medical graduates, The Association for Health Professional Education Conference, Australia.

Stokes, D., \& Fuller, T. (2009) Review of internet-based psychological services and products. Health Beyond (e-Health conference), Australia.

Harris, A., Fuller, T., \& Petrov, I. (2009) Expand your horizons: a lecture series by International Medical Graduates. Department Human Services - Showcase: A world of expertise, Australia.

\section{SELECTED RECENT POSTERS}

Lourenco, MPCG, Fuller TE, Vlaeyen, JWS \& Cima, RFF (2018) What was that? Utilizing innovative methodology to break down cognitive behavioural therapy for tinnitus. $N=1$ Stockholm Symposium, Stockholm, Sweden.

Fuller, T., Cima RFF, Van den Bussche, E., \& Vlaeyen JWS (2016) Psychometric properties of the Fear of Tinnitus Questionnaire. $10^{\text {th }}$ International Tinnitus Research Initiative Conference, Nottingham, England.

Cima RFF, Fuller, T., Van den Bussche, E., \& Vlaeyen JWS (2016) Psychometric properties of the Tinnitus Catastrophizing Scale. $10^{\text {th }}$ International Tinnitus Research Initiative Conference, Nottingham, England. 



\section{5.}
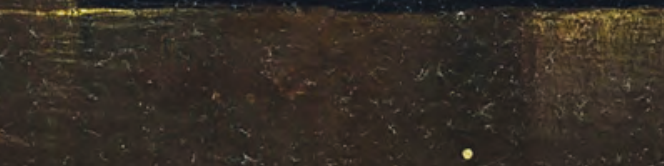

$t$

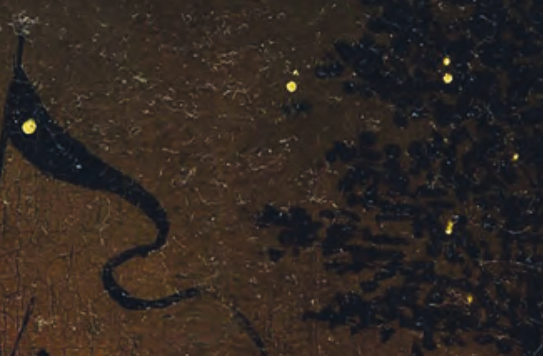

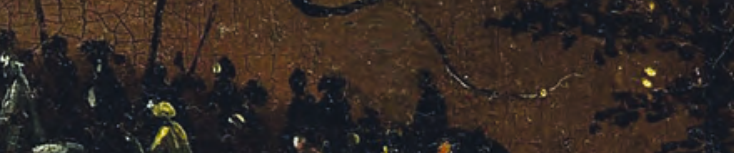

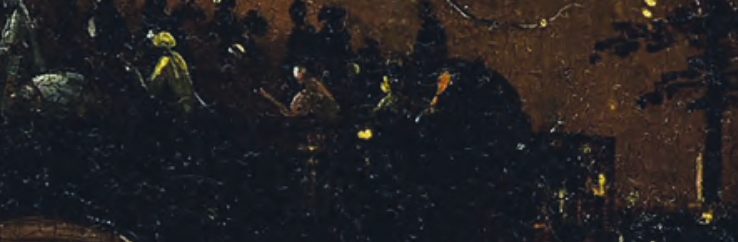

59

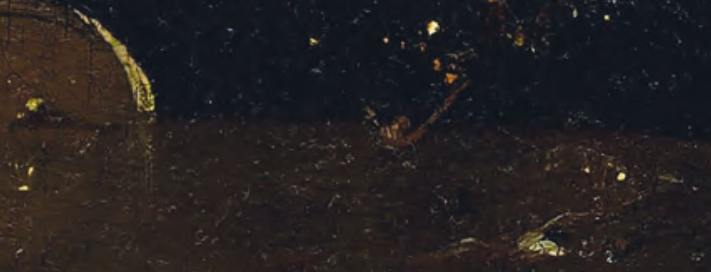

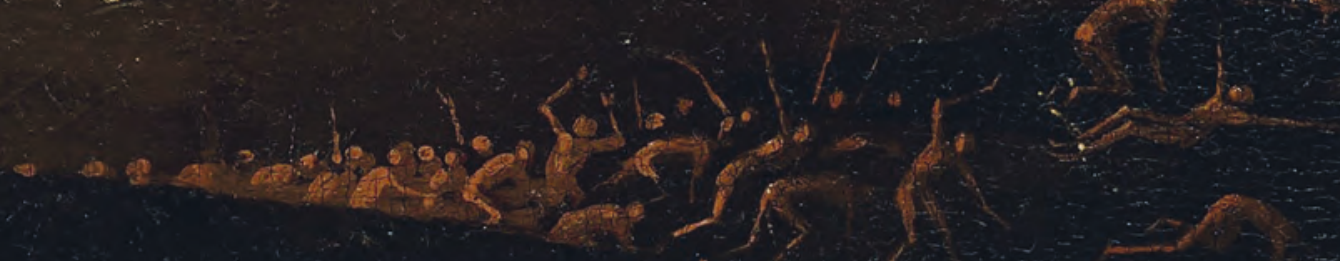

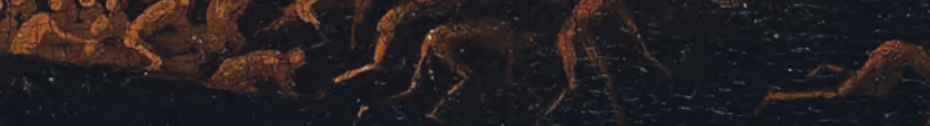

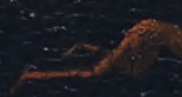

